Tumorßiology

\title{
Cancer Research, Tumor Markers, Clinical Oncology
}

Abstracts

The 35th Meeting of the International Society for Oncodevelopmental Biology and Medicine, ISOBM 2007

September 15-19, 2007, Prague, Czech Republic

Organizer

T. Zima, Prague 
The organizers would also like to acknowledge the following sponsors for their support of the ISOBM 2007 meeting

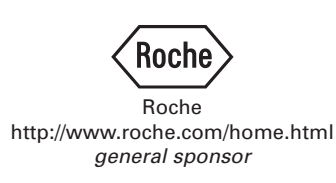

OLYMPUS

Olympus

http://www.olympus.com

main sponsor

Bayer HealthCare

Bayer Schering Pharma

Schering, s.r.o

http://www.bayer-cz.cz

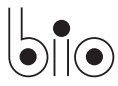

Consult

Bio-Consult, s.ro.

http://www.bioconsult.cz

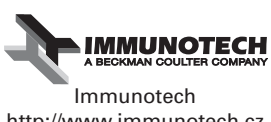

http://www.immunotech.cz

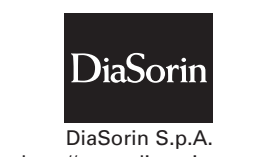

http://www.diasorin.com

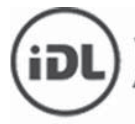

IDL Inc.

http://www.idl.se

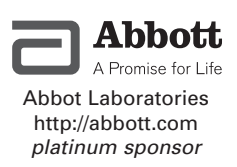

(l) NOVARTIS

Novartis

http://www.novartis.cz/en/index.htm

gold sponsor

\section{SIEMENS}

Siemens Medical Solutions Diagnostics, s.r.o. http://www.medical.siemens.com

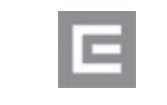

ČEZ, as.

http://www.cez.cz

\section{ZENTIVA}

Zentiva

http://www.zentiva.cz

\section{$\overbrace{\text { BECKMAN }}$ \\ COULTER}

Beckman Coulter, Inc. http://www.beckmancoulter.com gold sponsor

\section{ČESKÁத்} SPOŘITELNA

Česká spořitelna, a.s.

http://www.csas.cz

financial partner

\section{$B I O P A D$}

Bio-Rad, s.r.o.

http://www.bio-rad.com

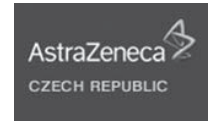

Astra Zeneca

http://www.astrazeneca.cz

B I๑VICA

Biovica

http://www.biovica.com

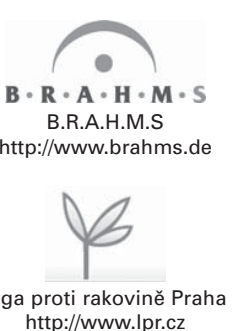

\section{S. Karger}

Medical and Scientific Publishers

Basel $\bullet$ Freiburg $\bullet$ Paris $\bullet$ London New York $\cdot$ Bangalore $\cdot$ Bangkok Singapore $\cdot$ Tokyo $\bullet$ Sydney
Disclaimer

The statements, options and data contained in this publication are solely those of the individual authors and contributors and not of the publisher and the editor(s). The appearance of advertisements in the journal is not a warranty, endorsement, or approval of the products or services advertised or of their effectiveness, quality or safety. The publisher and the editor(s) disclaim responsibility for any injury to persons or property resulting from any ideas, methods, instructions or products referred to in the content or advertisements.

Drug Dosage

The authors and the publisher have exerted every effort to ensure that drug selection and dosage set forth in this text are in accord with current recommendations and practice at the time of publication. However, in view of ongoing research, changes in government regulations, and the constant flow of information relating to drug therapy and drug reactions, the reader is urged to check the package insert for each drug for any change in indications and dosage and for added warnings and precautions. This is particularly important when the recommended agent is a new and/or infrequently employed drug.
All rights reserved.

No part of this publication may be translated into other languages, reproduced or utilized in any form or by any means, electronic or mechanical, including photocopying, recording, microcopying, or by any information storage and retrieval system, without permission in writing from the publisher or, in the case of photocopying, direct payment of a specified fee to the Copyright Clearance Center (see 'General Information').

(C) Copyright 2007 by S. Karger AG,

P.O. Box, CH-4009 Basel (Switzerland)

Printed in the Czech Republic by REPRO servis, Prague

ISBN 978-3-8055-8400-5

e-ISBN 978-3-8055-8401-2

\section{KARGER}


PLENARY LECTURES 
ALCOHOL AND CANCER

Helmut K. Seitz

Department of Medicine and Laboratory of Alcohol Research, Liver Disease and Nutrition, Salem Medical Centre, University of Heidelberg, Heidelberg, Germany

Compelling evidence exists in humans for a causal relationship between chronic alcohol consumption and the development of cancers of the upper gastrointestinal tract, the liver, the colorectum and the female breast.

There is overwhelming evidence that acetaldehyde is responsible for the carcinogenic effect of ethanol on the upper aerodigestive tract. Mechanisms of ethanol-induced hepatocarcinogenesis include cirrhosis of the liver, ethanol induced oxidative stress, altered methylation and a reduction of retinoic acid. An elevation of estradiols due to alcohol may contribute to breast cancer.

Considering the high frequencies of these cancers and the persistently high alcohol consumption in the general population, the link between alcohol and certain tumors has important consequences for prevention and early detection.

Some individuals should be considered at higher risk for alcohol-related cancers including those with a certain genetic predisposition (ALDH2*1/2 heterozygotes, possibly ADH1C*1/1 homozygotes and MTHFR 677 CT variants). Also, patients with chronic hepatitis $\mathrm{B}$ and $\mathrm{C}$, hereditary hemochromatosis and non-alcoholic fatty liver disease due to insulin resistance, GERD, and colorectal polyps are more susceptible towards the carcinogenic properties of ethanol.

Frequently encountered lifestyle factors such as smoking, poor oral hygiene, and certain dietary deficiencies (folate, vitamin B6, lipotropes) or excess of others (vitamin A/B-carotene) due to unevenly composed diets or self-medication also increase the risk for alcohol-associated tumors.

Although difficult to implement in clinical practice, alcohol consumption should be either completely avoided or strictly reduced in most of the situations described above.

\section{PL02}

\section{THERAPEUTIC STRATEGIES FOR CANCER STEM CELLS}

\section{Stewart Sell}

New York State Dept. of Health, Wadsworth Center, Empire State Plaza, Albany, NY, USA

Conventional cancer therapy is directed toward the transit amplifying cells of the cancer. Surgery removes them, chemo- and radiation therapy kills them, differentiation therapy forces their terminal differentiation and anti-angiogenic therapy cuts off their blood supply. Since cancers contain the same cell populations as normal tissues (stem cells, transit amplifying cells and terminally differentiated cells) treatment should be directed not only to the transit amplifying cells of the cancer, but also to the cancer stem cells. Studies of the prototype cancer, teratocarcinoma, demonstrate that the cancer transit amplifying cells can be forced to differentiate (differentiation therapy), but that the tumor mass will from cancer stem cells. Treatment of leukemias has a similar outcome. Killing the transit amplifying cells by chemotherapy or radiation, or inducing terminal differentiation by blocking of the constitutive activation of transit amplifying cells by specific inhibitors of signaling pathways effectively eliminates the transit amplifying cells of the leukemia. However, if the therapy is discontinued, the leukemia regenerates from leukemic stem cells. Epithelial cancers, such as skin, colon, liver and breast also arise from cancer stem cells and contain stem cells and transit amplifying cells. Identification of the cell signaling pathways of normal and cancer stem cells is the first step in designing inhibitors of cancer stem cells that may be effective in preventing regrowth of cancers from cancer stem cells. Inhibition of cancer stem cells may be mediated by molecular inhibitors that block cancer stem cell signaling pathways or by the use of small inhibitory RNA molecules (siRNA) that block synthesis of the signaling molecules. A critical problem is to design methods of delivery of potential inhibitory molecules to cancer cells in vivo. If these approaches ever become feasible, they could conceivably be used to prevent re-growth of cancers after conventional chemo- radiation or differentiation therapy, or force cancer stem cells to differentiate. However, direction of stem cell therapy may be complicated by the heterogeneity of the cells in a cancer. Ongoing studies in our laboratory on cells cultured from primary breast cancers in mice suggest that these cancers either contain more than one type cancer stem cell. 


\section{A PERSONALIZED APPROACH TO CANCER TREATMENT: HOW BIOMARKERS CAN HELP}

M J Duffy

St. Vincent University Hospital, Dublin, Ireland

The current approach to administering systemic therapy to cancer patients is largely empirical. Consequently, it is likely that many patients with aggressive disease are undertreated while many with indolent disease are overtreated. In addition, for those patients who receive treatment, only a proportion derives clinical benefit while many suffer from adverse side effects. Most serious of all, a small number suffer from severe toxic effects, which in very rare cases, may be fatal.

With recent developments in targeted therapies and molecular diagnostics, we are beginning to move from the traditional "trial and error" approach to a position involving a personalized approach, i.e., giving the right drug at the right dose to the right patient. In order to achieve this situation, we need:

- Strong and independent prognostic markers that can separate patients with indolent disease from those with aggressive forms,

- Markers to prospectively predict response or resistance to specific therapies in order that the right patients receives the right drug(s),

- Markers to identify patients likely to develop severe toxic side effects from therapy under consideration for administration.

The aim of this presentation is to review how advances in prognostic, predictive and toxicity markers are likely to lead to a personalized approach to cancer management.

PL04

ATTRACTIN, NEURODEGENERATION AND GLIOMA DEVELOPMENT

Jonathan S. Duke-Cohan ${ }^{1,2}$, Fatima H. Khwaja ${ }^{3}$, Erwin G. van Meir ${ }^{3}$

${ }^{1}$ Dana-Farber Cancer Institute, Department of Medical Oncology, HIM423, 77 Avenue Louis Pasteur, Boston MA 02115, USA, ${ }^{2}$ Harvard Medical School, Department of Medicine, Boston MA 02115, USA, ${ }^{3}$ Winship Cancer Institute, Emory University, Atlanta GA, USA

Attractin, initially discovered as a primate-specific circulating protein with chemotactic regulatory function, is in fact highly-conserved as a transmembrane protein with likely vesicular location in all metazoan species. The human secreted form, corresponding to the complete ectodomain of the transmembrane isoform, is the result of alternative splicing. During $\mathrm{T}$ lymphocyte activation, the ratio of membrane to secreted form mRNA altered in favour of the latter, and secretory function in general was associated with upregulation of secreted attractin. Conversely, in the central nervous system (CNS), neural differentiation was associated with a complete switching off of secreted attractin, and secreted form mRNA was essentially absent in the adult human CNS. Membrane form attractin is essential for CNS development in rodent models, and its absence leads to hypomyelination and spongiform neurodegeneration. This is corrected entirely by transgenic expression of the membrane but not the secreted form. The consensus is that the secreted form plays a role in the periphery, the membrane form is essential for CNS development and that inappropriate expression of the secreted form in the CNS may be detrimental. In this review, we will expand upon results demonstrating the cerebrospinal fluid expression of secreted attractin is highly upregulated as astrocytomas develop, that astrocytomas are the source of secreted attractin, that attractin helps metastatic spread and that its CSF levels may be used as a diagnostic marker. We examine the relationship of the secreted to membrane isoforms and show that inappropriate expression of CNSsecreted attractin by gliomas, by multiple-sclerosis inflammatory foci, and in AIDS with CNS involvement may contribute towards neurodegeneration. 


\section{PL05}

\section{ALPHA FETOPROTEIN - ITS 50 ${ }^{\mathrm{TH}}$ ANNIVERSARY}

Masopust, J., Kithier, K., Rád1, J.

$2^{\text {nd }}$ Faculty of Medicine, Charles University, 15006 PRAGUE 5, V Úvalu 84, CZ

The year of alpha fetoprotein (AFP) discovery can be marked as 1956, when Bergstrand and Czar reported their finding

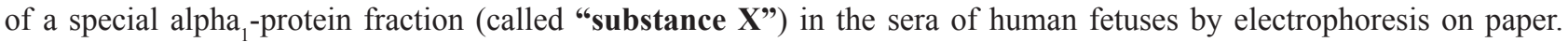
This finding was confirmed by de Muralt and Roulet (1961) and by us (Masopust 1962,1965) using immunoelectrophoresis; we named this specific fetal component fetoprotein. In 1963, Abelev et al. detected embryo-specific alpha ${ }_{1}$-globulin in

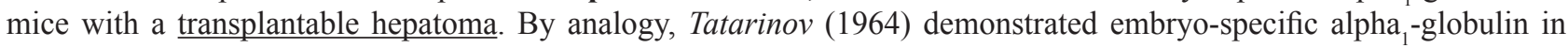
a case of human liver-cell carcinoma. In 1966, an abnormally increased concentration of AFP was reported in the sera of children with embryonic cell carcinoma of testicles and ovaries, in a boy with primary hepatoma and also in infants suffering from benign liver diseases (Masopust et al., 1966, 1968). In the second half of the sixties, intensive studies of AFP and of other fetal proteins became the focus of several experimental and clinical laboratories over the world. The number of publications with the term "fetoprotein" in the bibliographic databases (PubMed, NHL) rose up to 14785 within 30 years. In the year 2006 alone, there were 494 publications dealing with this topic. The AFP research and its application in diagnostics, therapy, and functional proteomics still remain very important. The topics, which we consider should receive special attention are the following: (1) Standardization of the AFP determination; (2) Selection of the most relevant criteria for screening, differential diagnosis, prognosis and for follow-up of the efficacy of the treatment of livercell carcinomas and also of other AFP positive tumors; (3) The use of APF, of its fragments and variants for the treatment of AFP positive tumors; (4) Research on AFP as a signal molecule within the cellular activities;

(5) Elucidation of some other biological AFP functions during ontogenesis.

PL06

\section{FINDINGS OF MALIGNANT TUMORS IN EUROPE AND ANCIENT EGYPT IN A DIACHRONIC PERSPECTIVE}

\section{Eugen Strouhal, Alena Němečková}

Charles University in Prague, First Faculty of Medicine, Institute for History of Medicine and Foreign Languages Charles University in Prague, Faculty of Medicine in Pilsen, Institute for Histology and Embryology, Czech Republic

For the first time in palaeopathology, we decided to attempt a diachronic elaboration of occurrence of findings of malignant tumors in the past. We used our largest database of 188 sufficiently documentated finds of malignant tumors from the Old World. It contains cases of tumors with 12 diagnoses joined into five diagnostic groups to be dealt with in the analysis. The findings originate in archaeological sites of 26 countries or regions, in which they are unevenly represented. Diachronic growth of them could be recorded separately by 113 cases from Europe and by 52 cases from Ancient Egypt and Nubia. The comparison revealed outstanding differences between these regions. In Europe, finds of malignant tumors were rare in prehistoric times since the Neolithic Period and their number stagnated. After the beginning of our era, they started to grow fast with a culmination in the High Middle Ages. On the other hand, first identification of tumors occurred in Egypt and Nubia at the Predynastic Period with a trend increaseing up until the peak in Late Period to Ptolemaic Period. After 1500 years AD in Europe and with the beginning of our era in Egypt and Nubia, there was a decrease tumors found, caused by an insufficient number of investigated cemeteries.

Key words: palaeopathology, finds of malignant tumors, geographical distribution, diachronic growth 
SYMPOSIA 


\title{
S1_A1
}

\section{GENE METHYLATION IN CANCER: OVERVIEW}

\author{
Manel Esteller \\ Director, Cancer Epigenetics Laboratory, CNIO, Spanish National Cancer Center, Barcelona, Spain
}

We are in an era where the potential exists for deriving comprehensive profiles of DNA alterations characterizing each form of human cancer. DNA methylation is the main epigenetic modification in humans. Tumor cells show aberrant methylation of several $\mathrm{CpG}$ islands, but global demethylation versus the counterpart normal cells. We have combined a candidate gene and biochemical approach to determine the overall aberrant DNA methylation in transformed cells. Our results show that $\mathrm{CpG}$ island promoter hypermethylation has a tumor-type specific pattern, where each gene tends to be methylated in the cancer cells driven from a particular tissue but not from others. Epigenetic silencing affects all cellular pathways: DNA repair (hMLH1, MGMT, BRCA1), cell cycle (p16 ${ }^{\mathrm{INK} 4 \mathrm{a}}, \mathrm{p} 14^{\mathrm{ARF}}, \mathrm{p} 15^{\mathrm{INK} 4 \mathrm{~b}}, \mathrm{p} 73$ ), apoptosis (DAPK, TMS1), hormone receptors (ER, PR, AR, RARB2, CRBP1), cell adherence (CDH1, TIMP3), detoxifiers (GSTP1) and many more (APC, LKB1, SOCS-1...). Promoter hypermethylation of particular genes have important consequences for the biology of that particular tumor. This is for example the case of the DNA repair gene MGMT which methylationmediated silencing leads to transition mutations, but, at the same time, "marks" those neoplasms that are going to be more sensitive to the chemotherapy with alkylating drugs. Hypermethylation can be observed in hereditary tumors, where it may account for the "second hit" of the tumor suppressor gene. We have also developed massive genomic screenings to find new hypermethylated genes in cancer cell. From these assays we have identified new candidate tumor suppressor genes with important potential roles in the pathogenesis of human cancer.

Second, we have studied the global methylcytosine content of a large collection of normal tissues and sporadic and hereditary primary tumors. The picture that emerges shows that $85 \%$ of human cancer cells are hypomethylated when compared to the original normal cells. We have also found that the 5-methylcytosine DNA content and the number of $\mathrm{CpG}$ islands hypermethylated in a given tumor is not random, but it involves environmental factors and genetic predisposition.

Overall, our data demonstrates that human tumors suffer a profound, but specific, disturbance in their DNA methylation and chromatin patterns.

S1_A2

METHYLATED GENES AS PROGNOSTIC AND PREDICTIVE MARKERS IN BREAST CANCER

John W.M. Martens

Department of Medical Oncology, Josephine Nefkens Institute, Erasmus MC, PO Box 2040, 3000 CA, Rotterdam, The Netherlands.

Introduction: Methylation of $\mathrm{CpG}$ islands in the promoter regions of genes is a prominent epigenetic silencing mechanism, which can be a driven force in breast cancer progression and/or a cancer's sensitivity to treatment.

Methods: Using various micro-array-based methodologies, we performed candidate and genome-wide DNA methylation profiling in primary tumors of breast cancer patients and associated differential methylation to disease outcome. We associated DNA methylation of candidate genes with disease recurrence in patients who received adjuvant tamoxifen therapy. And, in two other studies, we associated DNA methylation markers measured in the primary tumor of patients to response of their recurrent disease to respectively endocrine and anthracyclin-containing chemotherapy.

Results: (i) DNA methylation of PITX2 was associated with disease recurrence in node-negative patients receiving adjuvant endocrine therapy and in patients not receiving any adjuvant systemic therapy. (ii) DNA methylation markers PSAT1, STMN1, GRIND2, TGFBR2 and S100A2 were associated with first-line tamoxifen therapy response. (iii) The genome-wide discovery revealed various DNA methylation markers associated with anthracyclin-based chemotherapy response. These latter markers were further studied in a cohort of lymph node-positive patients who all received adjuvant anthracyclin containing chemo-therapy. Among others BMP4 was identified as the strongest marker for disease progression in this latter cohort.

Conclusion: Specific epigenetic DNA methylation markers were revealed associated with clinical outcome in patients treated with endocrine and chemotherapy during early stage and recurrent breast cancer. 
S1_A3

\title{
SERUM ASSESSMENT OF GENE PROMOTER REGION METHYLATION FOR THE EARLY DETECTION OF PROSTATE CANCER
}

Herbert Fritsche, Li-Ying Yangand Richard Babaian

MD Anderson Cancer Center, Lab Med Box 37, Houston, TX 77055, USA

\begin{abstract}
Aims
Aberrant methylation of gene promoter regions is a frequent event in human cancers. We investigated hypermethylation status of selected tumor suppressor genes in serum obtained from patients with prostate cancer.

Methods

Using methylation specific PCR, gene promoter methylation was analyzed in DNA extracted from serum of 73 men with prostate cancer and 67 biopsy-negative men.

Results

The methylation frequencies of 5 genes in the 52 cancer patients were: $\boldsymbol{G S T P 1}, 85 \% ; \boldsymbol{R A S S F 1 A}, 73 \% ; \boldsymbol{R A R} \beta, 56 \%$; $\boldsymbol{A P C}, 60 \%$; and $\boldsymbol{C D H 1}, 75 \%$. The methylation frequencies in the 35 non-cancer controls were significantly lower. The methylation status of the 3 most frequently methylated genes (GSTP1, RASSF1A and $C D H 1)$ including an additional set of 53 specimens (21 cancer and 32 non-cancer) were $77 \%, 67 \%$ and $78 \%$ for GSTP1, RASSF1A and CDH1, respectively. The methylation index (MI), defined as the fraction of the 3 genes methylated, for the cancer and non-cancer groups were $0.74 \pm 0.028$ and $0.25 \pm 0.023$, respectively. Using a cut-off value of $\geq 0.67$ for MI, we obtained a true positive rate and true negative rate of $86.3 \%$ and $82.1 \%$, respectively, regardless of the PSA range, and $82.7 \%$ and $82.1 \%$, respectively, among the subgroup of patients with low PSA levels $(<10 \mathrm{ng} / \mathrm{ml})$. The MI score was not correlated with the pathologic stage, Gleason score and preoperative serum PSA.

Conclusions

Methylation status of GSTP1,RassF1A and CDH1 gene promoter regions can be assessed in blood, and the frequency of gene methylation predicts the presence of prostate cancer with high sensitivity and specificity.
\end{abstract}




\title{
ORIGIN AND EVOLUTION OF CHILDHOOD LEUKEMIA
}

Oskar A. Haas

St. Anna Children's Hospital, Vienna, Austria

Childhood acute lymphoblastic leukemia (ALL) comprises a heterogeneous group of diseases. They are characterized by unique clone- and disease-specific abnormalities that derive from physiological developmental gene rearrangement and mutation-induced transformation processes. There is accumulating and compelling evidence that leukemia development is a multi-step process. In the majority of cases, this process sets off already prenatally during early fetal development. One or several subsequent hits trigger then the clonal expansion and progression to the clinical overt hematologic disease. The supporting evidence for this model derives primarily from the analysis of disease-specific as well as clone-specific gene rearrangements in identical twins, in neonatal blood spots and from comparing the respective patterns in diagnostic and relapse samples. Of particular interest in this context are the acquired chromosome translocations, such as the $\mathrm{t}(4 ; 11)$ and the $\mathrm{t}(12 ; 21)$ and their underlying chimeric fusion genes, MLL/AF4 and TEL/AML1. They provide unique, stable, and sensitive markers for each patient's leukemic clone. In addition to these disease-initiating abnormalities, the vast majority of the B- and T-cell precursor ALLs also harbor disease-inert clonotypic immunoglobuline and T-cell receptor gene rearrangements. We will describe how the unique patterns of these rearrangements are utilized to retrospectively determine the time of the initiation as well as to define the sequence of events during the development of these leukemias. Moreover, we will review the most striking results obtained with these analysis and their intriguing implications for the understanding of leukemogenesis in general.

S2_A2 ROLE OF C-KIT GENE IN CORE BINDING FACTOR LEUKEMIA: FROM THE MUTATIONS TO THE
PATHWAYS AND THERAPEUTICS

\author{
Lidia Larizza \\ Medical Genetics, San Paolo School of Medicine, University of Milan, Via A. di Rudini 8, 20142 Milano, Italy
}

The c-kit gene is a member of the type III receptor tyrosine kinase family and plays a crucial role in normal hematopoiesis and acute myeloid leukaemia (AML) (1).

Different gain-of-function mutations in the KIT juxtamembrane and tyrosine kinase domains were identified in about $46 \%$ of patients with a distinct subgroup of AML, with common clinical features and outcomes, defined as Core Binding Factor Leukemia (CBFL)(2-4). According to the stepwise model of leukemogenesis (5) genetic abnormalities in the c-kit gene are considered a second but crucial hit for development of a full-blown leukaemia conferring autonomous proliferation and anti-apoptotic effects to myeloid blasts impaired in differentiation by $\mathrm{t}(8 ; 21)$ and inv(16) chromosomal rearrangements. Functional consequences of these activating mutations on KIT-mediated signal transduction pathways are recapitulated, with focus on the tyrosine kinase Asn822Lys mutation of the Kasumi-1 CBKL cell line $(6,7)$ in which KIT downstream signaling has been investigated by means of STI 571 pharmacological inhibition (8). Search of kitpositive AMLs is currently applied in the clinical set as it provides a prognostic view in association with the decision to administer to selected patients carrying responsive mutations specific tyrosine kinase inhibitors.

1) Ratajczak M et al, Proc Natl Acad Sci USA 1992, 89: 1710-1714

2) Beghini A et al, Blood 2000, 95(2):726-7

3) Beghini A et al, Haematologica, 2004, 89: 920-925

4) Cairoli R et al, Blood, 2006, 107(9): 3463-8

5) Gilliland DG, Tallman MS, Cancer Cell. 2002, 1(5):417-20.

6) Beghini A et al, Hematol J. 2002, 3(3):157-63.

7) Larizza et al, Leuk Lymphoma 2005, 46: 247-255

8) Beghini et al., Exp. Hemat., 2005, 33(6):682-8 


\title{
THE PRINCIPLE ROLE OF ILLEGITIMATE IG REARRANGEMENTS IN PATHOGENESIS OF B-CELL MALIGNANCIES
}

\author{
Wlodarska Iwona \\ Center for Human Genetics, KU. Leuven, Belgium
}

Chromosomal translocations hallmarking B-cell lymphoma mostly involve the immunoglobulin (Ig) genes and a variety of partner genes. Molecularly, these aberrations lead to a transcriptional dysregulation of the affected proto-oncogenes by their juxtaposition with regulatory elements of Ig. Prominent examples include $\mathrm{t}(14 ; 18)(\mathrm{q} 32 ; \mathrm{q} 21) / \mathrm{Ig}-\mathrm{BCL} 2, \mathrm{t}(11 ; 14)(\mathrm{q} 13 ; \mathrm{q} 32) /$ Ig-CCND1 and $\mathrm{t}(8 ; 14)(\mathrm{q} 24 ; \mathrm{q} 32) / \mathrm{Ig}-\mathrm{CMYC}$ characteristically associated with follicular lymphoma, mantle zone lymphoma and Burkitt lymphoma, respectively. Mechanisms underlying Ig-related translocations are associated with somatic Ig remodeling processes. These processes operate on different steps of normal B cells development and include $\mathrm{V}$ gene segment assembly, somatic hypermutation, and class switch recombination. Illegitimate Ig rearrangements possibly play also a causative role in generation of interstitial deletions of $14 \mathrm{q}$ involving $\operatorname{IgH}(14 \mathrm{q} 32.33)$. This novel type of chromosomal aberrations was very recently identified by us in B-cell malignancies using array comparative genomic hybridization $(\mathrm{aCGH})$ (1). Further screening by fluorescence in situ hybridization (FISH) detected these deletions in altogether 30 cases of B-cell leukemia/lymphoma. As shown by aCGH and FISH, the size of these deletions ranged in 9 cases and involved the same 14q24.13q32.33 region in 21 cases. Interestingly, the latter deletion was predominantly observed in chronic lymphocytic leukemia. Given a constant involvement of IgH, we hypothesized that these interstitial del(14) may operate in a translocation-like manner bringing unknown 14q protooncogene(s) in a vicinity of the powerful IgH enhancer. So far, the gene targeted by the recurrent del(14)(q24.13q32.33) remains unknown. Further investigations are needed to unravel the mechanism(s) and role of IgH-involving del(14)(q) in B-cell malignancies.

Acknowledgements.

This work was supported by the KULeuven Research Foundation (BIL03/12 and BIL 05/59) and grant G.0610.07 from the Fund for Scientific Research (FWO), Flanders, Belgium.

S2_A4

\section{COMPLEX DIAGNOSTIC APPROACH TO NON-HODGKIN LYMPHOMAS IN HANDS OF PATHOLOGISTS AND MOLECULAR BIOLOGISTS}

Roman Kodet, Helena Břízová, Vít Campr, Markéta Kalinová, Lenka Krsková, Petra Mandáková, Marcela Mrhalová

Department of Pathology and Molecular, Medicine Faculty Hospital Motol and 2nd Faculty of Medicine Charles Univ. Prague, Czech Republic

Diagnostics of lymphomas is easier at present than in the past due to the international unification of terminology and characteristic features (WHO classification). The behavior of various entities is predictable in general but there is still a great variation in individual patients due to different properties of the tumor cell populations.

There are two major aims of interest: i), to identify reliable features of individual lymphomas that would separate those more favorable from those progressing more rapidly and ii), to quantify the tumor load and its response to the therapy. This may be achieved through monitoring the disease in the bone marrow using quantitative PCR.

For example, in mantle cell lymphomas (MCLs) the best prognostically informative test is to establish the tumor proliferation rate. In diffuse large B-cell lymphomas (DLBCLs), separation of subtypes arising from germinal centers and subtypes generated from activated B-cells is crucial to disclose at least two or more separable subgroups with a different prognosis. In peripheral T cell lymphomas (PTCL) the situation is still unclear. Generally, the prognosis of PTCL is unfavourable except for anaplastic large cell lymphomas (ALCLs) positive for anaplastic lymphoma kinase (ALK).

We demonstrate usefulness of the disease monitoring on two models. In both a characteristic molecular marker of the tumor cell population is identified. In MCL a product of $t(11 ; 14)$ leads to overexpression of cyclinD1. CyclinD1 is an appropriate target for the lymphoma monitorig. In ALCLs a fusion gene ALK-NPM and its transcript is used to monitor the disease development.

Supported by MZO-00064203/6704 and MSM-00216813. 


\title{
MOLECULAR CYTOGENETICS IN DIAGNOSTICS OF LEUKEMIA
}

\author{
Zemanová Zuzana and Michalová Kyra
}

The Center of Oncocytogenetics, Institute of Clinical Biochemistry and Laboratory Diagnostics, General University Hospital and $1^{\text {st }}$ Faculty of Medicine, Charles University in Prague, U Nemocnice 2, 128 08, Prague 2, Czech Republic

Tumor origin and evolution is series of specific genetic events that take place in a target cells and their clonal descendants. These events can be recognized at two broad levels: cytogenetic and molecular. With classical cytogenetic methods many recurrent and specific chromosomal changes were ascertained and became important genetic markers which are used as indispensable laboratory indicators for the clinical examinations at the diagnosis of malignant disease. During the time the knowledge of these markers, and many new ones, improved our diagnostic and prognostic ability specially in oncohematology. Furthermore, chromosome analysis of hematologic malignancies and some of the solid tumors has provided critical insights into the genetic changes that underlie malignant transformation of the cells. Molecular cytogenetic methods have led to the identity of genes and their precise localization on chromosomes and contributed to the finding of further, some rather cryptic rearrangements. Molecular geneticists then cloned translocation, inversion breakpoints and regions of cryptic deletions and derived DNA probes. When labeled with fluorochromes, DNA probes can be used for fluorescence in situ hybridization (FISH) and can precisely identify the presence, absence or amplifications and rearrangements of specific genes both in interphase nuclei and in metaphase chromosomes. Pathological clone can be followed by FISH quantitatively during the therapy and can serve as indicator of its efficiency. Recurring chromosomal abnormalities are associated with distinct subtype of leukemia and lymphoma with unique morhologic, immunophenotypic and clinical features. Conventional and molecular cytogenetic analyses of leukemic cells have led to the definition of prognostic risk groups and the development of subtype-specific or risk-adapted therapy strategies and have contributed important informations to the understanding of pathogenetic mechanisms in leukemogenesis.

Supported by grants MSM 0021620808, MSM 0021620813, MZO 00064165, MSM LC535 and NR/9227-3.

S2_A6

\section{PAX5 PROMOTES LYMPHOMAGENESIS THROUGH THE STIMULATION OF B-CELL RECEPTOR SIGNALING}

Diana Cozma, Duonan Yu, Suchita Hodawadekar, Anna Azvolinsky, Shannon Grande, John W. Tobias, Michele H. Metzgar, Jennifer Paterson, Jan Erikson, Teresa Marafioti, John G. Monroe, Michael L. Atchison, and Andrei Thomas-Tikhonenko

Department of Pathobiology, University of Pennsylvania, 3800 Spruce Street, Philadelphia, PA 19104-6051, USA

The presumed involvement of the PAX5 gene in B-lymphomagenesis is based largely on the discovery of Pax5-specific translocations and somatic hypermutations in diffuse large B-cell and other non-Hodgkin lymphomas. Yet at the molecular and cellular levels, the contribution of Pax 5 to neoplastic growth remains undeciphered. Here we took advantage of two Myc-induced B-cell lymphoma cell lines (dubbed Myc5-M5 and -M12) that spontaneously silence Pax5 and form indolent tumors of undifferentiated phenotype. Reconstitution of these cells with Pax5-tamoxifen receptor fusion greatly boosts neoplastic growth in the hormone-dependent manner. Conversely, the expression of dominant-negative Pax 5 in murine lymphomas or Pax 5 knockdown in human lymphoma cells negatively affects cell expansion. Expression profiling revealed that Pax5 is required to maintain expression of several crucial components of B-cell receptor (BCR) signaling, including CD79a, a protein with the immunoreceptor tyrosine-based activation motif (ITAM). In contrast, expression of two known ITAM antagonists, CD22 and PIR-B, was suppressed. The key role of BCR/ITAM signaling in Pax5-dependent lymphomagenesis has been corroborated in the following experiments. First, constitutively active ITAM enhanced tumorigenesis by Pax5-negative cells. Second, Pax5-dependent lymphomagenesis was abrogated by forced expression of CD22 and by a pharmacological inhibitor of Syk, a ITAM-associated tyrosine kinase. Third, immunohistochemical studies revealed consistent expression of phosphorylated BLNK, a BCR effector, in human B-cell lymphomas. Thus, stimulation of neoplastic growth by Pax 5 occurs through BCR and is sensitive to genetic and pharmacological inhibitors of this pathway. 


\section{PROGNOSTIC SIGNIFICANCE OF CYTOKERATINS - TPS, TPA - IN BREAST CANCER}

Vivian Barak, Beatrice Uziely, Benjamin Nisman, Ayala Hubert, Inna Kalickman, Tamar Peretz

Immunology Lab for Tumor Diagnosis, Oncology Dep., Hadassah Hebrew University

Medical Center, Jerusalem, Israel

STUDY AIMS: To evaluate the prognostic potential of Cytokeratin Tumor Markers-TPA, TPS, compared to tumor mass markers (CA15-3, CA125, CEA) and clinical status.

METHODS AND PATIENTS: The study consisted of 187 advanced Breast Cancer patients (pts), followed up for 7 years by tumor markers and correlated to clinical parameters.

RESULTS: A significant correlation of TPS with response to therapy, early detection (lead time) of remissions or recurrences as well as survival, were demonstrated. Univariate analysis of pretreatment marker levels (accepted cut-off levels) showed significance for TPA,TPS, CEA, CA125 and CA15-3. Median survival time of pts with low levels of CA 125, TPS, TPA, CA15-3 and CEA were $23 \mathrm{~m}, 18 \mathrm{~m}, 16.5 \mathrm{~m}, 12 \mathrm{~m}, 9.6 \mathrm{~m}$ as opposed to high marker levels; $8.8 \mathrm{~m}, 9 \mathrm{~m}, 8.2 \mathrm{~m}$, $7.4 \mathrm{~m}, 9.7 \mathrm{~m}$, respectively. Survival was best correlated to low CA 125 and TPS entering levels. CA125 and TPS retained significance also in multivariate Cox's regression analysis.

CONCLUSIONS: We conclude that the cytokeratin marker TPS (as CA 125), provide the most important information for prognosis in advanced breast cancer pts.

S3_A2

\section{ONCOLOGICAL BIOMARKERS IN THE EARLY DETECTION OF METASTATIC BREAST CANCER}

$\underline{\text { Petra Stieber }^{1}}$, Ingo Bauerfeind ${ }^{2}$, Dorit Lässig ${ }^{3}$, Dorothea Nagel ${ }^{1}$, Volker Heinemann ${ }^{3}$

${ }^{1}$ Institute of Clinical Chemistry, Klinikum der Universität München-Großhadern-, Marchioninistraße 15, 81377 München, Germany

${ }^{2}$ Gynecological Department, Klinikum der Universität München -Großhadern-

${ }^{3}$ Medical Department III, Klinikum der Universität München -Großhadern-

Aims:

CEA and CA 15-3 are often determined during follow up care of breast cancer, but due to a lack of knowledge, experience and confidence- the actual situation is more to observe than to react on the values in this clinical situation of asymptomatic patients and no evidence of disease. We validated the diagnostic capacity of CEA and CA15-3 in the early detection of metastatic breast cancer.

Methods:

After complete removal of the tumor (R0 resection) patients will reach again their individual "normal" values, the basis for follow up care. Breast cancer patients reach 4 weeks after the end of adjuvant radio/chemotherapy median values for CEA (Abbott, AxSYM) and CA 15-3 (Roche, Elecsys) comparable to the medians of healthy individuals (CEA: $1 \mathrm{ng} / \mathrm{ml}$; CA 15-3: $13 \mathrm{U} / \mathrm{ml})$.

Results:

899 breast cancer patients with a non metastatic primary tumor and available individual baseline values after primary treatment were included. Of these 717 patients remained disease-free (control group), whereas 182 patients developed metastatic disease during follow-up

If we postulate an increase of $100 \%$ for the early detection of metastatic breast cancer a specificity of $99 \%$ for both biomarkers and a sensitivity of $14 \%$ for CEA, $23 \%$ for CA $15-3$ and another $27 \%$ for both, resulting in $36 \%$ false negatives. If fixed cut off values are used (CEA: $4 \mathrm{ng} / \mathrm{ml}, \mathrm{CA} 15-3: 30 \mathrm{U} / \mathrm{ml}$ ) 22\% false positives (specificity 78\%) and a sensitivity of $8 \%$ for CEA, $29 \%$ for CA $15-3$ and another $26 \%$ for both resulting in $37 \%$ false negatives. Using higher cut off values where the probability of metastatic breast cancer is high (CEA: $7 \mathrm{ng} / \mathrm{ml}$, CA 15-3: $69 \mathrm{U} / \mathrm{ml}$ ) a specificity of $96 \%$ is reached, but the sensitivity for CEA is 7\%, for CA $15-319 \%, 12 \%$ for both (58\% false negatives).

Conclusion:

Thus in follow up care the use of kinetics based on individual baseline values shows by far the best profile of specificity and sensitivity. 


\section{CYTOKERATINS AS MARKERS FOR COLORECTAL CARCINOMA}

O. Topolcan, S Svobodova, L.Holubec ${ }_{\mathrm{jr}}$, V Treska., S Kormunda, A Sutnar, K Rupert, M. Prazkova, M. Casova V. Polivkova, M. Spisakova

Faculty Hospital in Pilsen, Dept. of immunoanalysis, E. Benese 13, 30599 Pilsen, Czech Republic

Aim of the studv: To confirm the clinical importance of TPS T colorectal cancer for the routine practice.

Methods: TPS was measured using IRMA method (IDL, Sweden), TK - REA method (Immunotech, Czech Republic) in serum in 654 patients. 445 patients with colorectal cancer were enrolled. They were also monitored during the $1.5-3$ year follow-up period of this disease.

The control group consisted of 200 patients. Other tumor markers (CEA, TK and CA 19-9) were measured at the same time.

Results: The sensitivity of TPS was 35\% and TK $38 \%$ during the preoperative period. TPS values correlated with TK levels and did not correlate with levels of CEA and CA 19-9. We have found that immediately after surgery there was an increase of TPS but a significant decrease of CEA. During the follow-up period TPS and TK usually increased $3-9$ months prior clinical symptoms of disease recurrence or in the case of the disease spread. Sensitivity of TPS 9, 6 and 3 months prior to clinical manifestation was 29,45 and $67 \%$. CEA sensitivity was only 10,25 and $40 \%$. Sensitivity of TPS at the time of clinical manifestation was $67 \%$, but the sensitivity of CEA at the same time was $72 \%$.

Conclusion: TPS is a predictive prognostic marker. Disease progression can be diagnosed prior clinical symptoms appearance using TPS and TK measurement. There is no clinical importance for their measurement immediately after surgery.

This study was supported by research project 0021620819 and NR 8301-3/2005.

S3_A4

\section{CLINICAL APPROACH OF CYTOKERATINS IN PATIENTS WITH LUNG CANCER}

R. Molina, J.M. Auge, X. Filella, R. Marrades, N. Viñolas

Oncobiology Unit, Hospital Clinic, Medical School, Hospital Clinic. Villarroel 170, Barcelona 08036, Spain

Tumor marker serum levels were prospectively studied in 267 patients with suspicious signs of lung cancer, being the final diagnosis, no- malignancy in 58 patients ( 15 infectious, 43 non infectious diseases), 28 patients with malignancies other than lung cancer and lung cancer in the remaining 181 patients (146 NSCLC, 35 SCLC). Slightly high abnormal serum levels were found in CEA 8,6\%, CA 19.9 and CA 125 in 22,4\%, NSE 0\%, CYFRA 3,4\%, TAG 12,1\%, SCC 1,7\%, CA 15.3 in $3,4 \%$ and TPA in $13,7 \%$ of the patients with benign diseases. Significantly higher concentrations of TPA, CA 19.9 and CYFRA 21.1 were found in patients with infectious diseases than in other benign pathologies. $(p=0,002,0,01$ and 0,02 , respectively).

Tumor marker sensitivity was related to cancer histology and tumor extension. Significantly higher NSE serum concentrations and sensitivity was found in SCLC than in NSCLC ( $\mathrm{p}=0,001)$. SCC, CEA, CA 15.3 and CA 125 were also related to the histological type, with significantly higher values in patients with NSCLC $(0,024,0,003,0,003$ and 0,012 , respectively). Cytokeratins, CYFRA and TPA, show a high concordance of the results in patients with lung cancer, in both NSCLC and SCLC (85,5\% and 91,4\%, respectively). The best combination of tumor markers are CEA, CA 15.3 and one cytokeratin in adenocarcinomas and CEA, SCC and one cytokeratin in squamous tumors. 


\section{PROGNOSTIC FACTORS IN OVARIAN CANCER}

Arie van Dalen*, J. Favier, E. Hallensleben, A. Burges, P. Stieber, HWA de Bruijn, D Fink, A. Ferrero, P. McGing, A. Harlozinska, Ch. Kainz, J. Markowska, R. Molina, C. Sturgeon, A. Bowman, R. Einarsson and H. Goike.

Institute of Tumor Marker Oncology, Van Stryenstraat 44, 2801 TG Gouda, The Netherlands as coördinator of the multicentre study.

Aims: A multicentre evaluation of predictive factors of 10 year OS in ovarian cancer patients including tumor marker values of CA 125 and TPS after 3 chemotherapy courses.

Methods: Kaplan Meyer survival curves and Cox Proportional Hazards Model in 212 patients (FIGO stage I-IV) receiving combined chemotherapy considering stage, histological type of the epithelial tumor, grade of differentiation, type of operation and CA 125 and TPS levels above and below optimal cut-off value after 3 chemotherapy courses as parameters.

Results: We have already published the results of 1 and 2 year OS. In patients with radical surgery (stage I; N=34 and stage II; $\mathrm{N}=23$ ) no difference in 10 year OS was observed. All seven patients with stage II and partial debulking died within 10 years. Grade 3 versus grade $1+2$ and clear cell + undifferentiated carcinoma versus other histological types were adverse predictive factors of 10 year OS in univariate analysis but not in multivariate analysis.

Univariate analysis of risk factors in stage III+IV $(\mathrm{N}=148)$ showed significant differences of i) optimal debulking versus partial debulking+staging laparotomy, ii) stage IV versus stage III and iii) tumor grade $2+3$ versus 1 . CA 125 levels $>25 \mathrm{kU} / 1$ and TPS $>100 \mathrm{U} / 1$ were adverse predictive factors of outcome. In multivariate analysis these factors were all independent. Only patients with optimal debulking $(\mathrm{N}=60)$ showed a reasonable survival $(23 \%)$. If both markers were elevated in this group survival was $0 \%(0 / 6)$ and with 1 marker elevated $10 \%(2 / 20)$. When both markers were below the cut-off levels survival was $35 \%(12 / 34)$.

Conclusions: CA 125 and TPS levels after 3 chemotherapy courses are highly predictive factors of 10 year OS in patients FIGO stage III+IV and optimal debulking.

References: A. Van Dalen et.al. Prognostic significance of CA 125 and TPS levels after 3 chemotherapy courses in ovarian cancer patients. Gyn Oncol 79, 444-450 (2000)

S3_A6

\section{USING SERUM CYTOKERATIN-18 ASSAYS TO DETERMINE CELL DEATH MODE}

$\underline{\text { Stig Linder }}$

Cancercenter Karolinska, R8:00, Karolinska Hospital, 17176 Stockholm, Sweden

Cytokeratin 18 (CK18) is cleaved by caspases during apoptosis. The molecular form of extracellular CK18 therefore reflects cell death mode: a high fraction of caspase-cleaved CK18 indicates apoptosis, the presence of mainly uncleaved CK18 indicates necrosis. Various observations, including measurements on tumor venous blood, suggest that serum caspase-cleaved CK18 is of tumoral origin. The levels of CK18-Asp396 and total CK18 has been measured in serum of prostate cancer and breast cancer patients during therapy. The results show that CK18 biomarkers are useful for prediction of the response to chemotherapy and are potentially useful biomarkers in clinical trials. The results also show that different chemotherapy modalities induce different death modes (apoptosis vs necrosis).

Kramer et al., Cancer Res 64, 1751-56, 2004; Kramer et al., Br J Cancer 94, 1592-8, 2006; Hägg Olofsson et al., Clin Cancer Res., accepted for publication) 


\section{HUMAN CHORIONIC GONADOTROPIN (HCG) ISOFORMS AND THEIR EPITOPES: PHYSIOLOGICAL OCCURRENCE AND DIAGNOSTIC ROLES IN PREGNANCY AND ONCOLOGY}

$\underline{\text { Peter Berger }}^{1}$, Cathie Sturgeon ${ }^{2}$, Jean-Michel Bidart ${ }^{3}$, Elisabeth Paus ${ }^{4}$, Adrian Bristow ${ }^{5}$, Christoph Zenzmair $^{1}$, Steven Birken ${ }^{6}$, Ulf-Hakan Stenman ${ }^{7}$

${ }^{1}$ Institute for Biomedical Aging Research, Austrian Academy of Sciences, Innsbruck, Austria

${ }^{2}$ Dept. of Clinical Biochemistry, Royal Infirmary, Edinburgh, United Kingdom

${ }^{3}$ Dept. of Clinical Biology, Institut Gustave-Roussy, Villejuif, France

${ }^{4}$ Central Laboratory, Norwegian Radium Hospital, Oslo, Norway

${ }^{5}$ National Institute of Biological Standards and Control, Potters Bar, Herts, United Kingdom

${ }^{6}$ College of Physicians and Surgeons of Columbia University, NY, U.S.A.

${ }^{7}$ Dept. of Clinical Chemistry, Helsinki University Central Hospital, Finland.

hCG and related variants play important diagnostic roles in pregnancy and oncology. In addition free hCG $\alpha$ and hCG $\beta$ subunits can be detected in both genders under physiological non-pregnant conditions. Comparability of immunoassay results for hCG and related markers has been limited by (i) lack of data regarding the molecular localization of epitopes recognized by monoclonal antibodies (mAbs) utilized in "hCG" immunoassays, (ii) method and antibody-related differences in the spectrum of molecular variants detected in different commercial immunoassays, and (iii) the molecular heterogeneity of analytes. The objective of this multi-center study (ISOBM TD-7 hCG Workshop) has been to improve comparability of diagnostic immunoassay results for "hCG". Molecular epitope assignment has been performed for $27 \mathrm{mAbs}$ from 7 international diagnostic companies. Antigens used included the new WHO-adopted first Reference Reagents ( ${ }^{\text {st }}$ IRR) for Immunoassay of hCG and five hCG-related variants (hCGn, hCG $\alpha$, hCG $\beta$, hCG $\beta \mathrm{n}, \mathrm{hCG} \beta \mathrm{cf}$ ), prepared under the auspices of the IFCC (International Federation of Clinical Chemistry). These are the first hormone standards calibrated in nanomolar concentrations by amino acid analysis. Recognized epitopes were (i) located on the first and third loops protruding from the cystine knot of hCG $\beta$ (aa $h C G \beta 20-25$ and 68-77), (ii) presumably centered around the knot itself, or (iii) on hCG $\beta C T P$ ( $h C G \beta 141-144$, or $h C G \beta 113-116$ ). For routine diagnostic purposes, assays recognizing a broad spectrum of $h C G+h C G \beta-$ variants such as $h C G+h C G n+h C G \beta+h C G \beta n+h C G \beta c f+-C T P h C G+-C T P h C G \beta$ may be useful. These criteria are best achieved by combinations of mAbs directed against epitopes located around the cystine knot (epitope $\beta_{1}$ ) and against those encompassing the top of loops 1 and 3 on hCG $\beta\left(\beta_{2}, \beta_{4}\right)$. In healthy probands networks of neuroendocrine cells and fibroblasts of the prostate and the testis synthesize hCG $\alpha$ and hCG $\beta$, which are then found in high concentrations in seminal plasma. Data indicate that hCG $\alpha$ and $\mathrm{hCG} \beta$ possess hCG-independent functions in male and female fertility and in particular suggest transcrine effects of male seminal plasma factors on the female reproductive tract. 


\title{
HCG STANDARDIZATION AND QUALITY CONTROL
}

\author{
C Sturgeon, P Berger, S Birken, J-M Bidart, R Norman and U-H Stenman. \\ C/o Dept of Clinical Biochemistry, Royal Infirmary of Edinburgh, Edinburgh EH16 4SA, UK.
}

Considerable scope exists for improving between-method comparability for hCG-related molecules, as is readily apparent from data from external quality assessment (EQA) schemes. In the UK National External Quality Assessment Scheme (UK NEQAS) for hCG, within-sample, between-laboratory geometric coefficients of variation (GCVs) have decreased somewhat over the last ten years. However, despite major technological advances, they remain high, varying for individual specimens from 11.6 to $18.9 \%$ in 2006 . The over-recovery (>110\%) of hCG IS 75/589 consistently observed for several major methods suggests that improved calibration would reduce this variation. Reflecting the characteristics of the antibodies employed, and also assay design, method mean results for samples containing purified hCG $\beta$ can vary by more than two-fold. EQA data also demonstrate differences in method precision, stability of results at low analyte concentration, and robustness to clinically relevant interferences (e.g. high dose hooking), all of which potentially have major implications, both for between-method comparability and for patient care.

International initiatives to improve the analytical comparability of assays for hCG are being actively supported by the International Federation of Clinical Chemistry and Laboratory Medicine (IFCC) and the International Society for Oncodevelopmental Biology and Medicine (ISOBM). Use of nomenclature developed by the IFCC Working Group for $\mathrm{hCG}$ and subsequently adopted by the IFCC should permit clearer description of what is being measured. The availability of highly purified International Reference Reagents for six important hCG-related molecules [intact hCG, nicked intact hCG (hCGn), hCG beta-subunit (hCG $\beta$ ), nicked hCG beta-subunit (hCG $\beta$ n), hCG alpha-subunit ( $\mathrm{hCG} \alpha$ ) and hCG betacore fragment (hCG $\beta \mathrm{cf})$ ], prepared by the same IFCC Working Group and calibrated in molar units by amino acid analysis, should enable improved understanding of what current hCG assays measure. Together with complementary results of ISOBM hCG antibody mapping studies, these activities should facilitate the development of improved hCG assays, ultimately benefiting patient care.

\section{S4_A3}

\section{HCG IN TESTICULAR AND OTHER MALIGNANT DISEASES}

\section{$\underline{\text { Rolf Lamerz }}$}

Rolf Lamerz, Med. Klinik II, LMU-Klinikum-Campus Grosshadern, Marchionini-Str. 15, D-81377 Muenchen, Germany

A overview is presented on HCG in cancer except for gestational trophoblastic diseases (GTD). HCG is known as one of the oldest and most specific tumor markers with different clinical application. Its broadest clinical usefulness is assessed in germ cell tumors (GCT) as most common cancer in younger males enabling a high rate of curability with long term remission in up to $90 \%$ by treatment with surgery, radiation and/or chemotherapy. As its prognosis and curability are highly dependent on TNM stage, number and extension of visceral metastases and initial concentrations of several tumor markers (TM) including hCG, AFP, LDH and PLAP, early clinical symptoms or suspicion require immediate initiation of clinical, laboratory and imaging investigations for achieving final diagnosis and appropriate treatment. The combination of a well detectable and effectively treatable tumor and the disposition of nearly ideal tumor markers hCG and AFP reflecting different components of tumor tissue (hCG for syncytiotrophoblastic, AFP for yolk sac elements) underscore their multifold utility for diagnosis, prognosis, response of therapy and recognition of relapse especially for nonseminomatous GCT $(\mathrm{n}=147)$ and the high risk subgroup of patients with very high HCG levels $(\mathrm{n}=52)$. Concerning non-trophoblastic cancers, hCG is mainly expressed as hCGß subunit and rarely as intact hCG, nicked forms or hCGßcf (predominant urinary form) in tissue (immunohistochemistry, RT-PCR mRNA), serum, serous effusions and in urine and mostly used as pretherapeutic prognostic marker. Main examples provided concern bladder, renal, prostate, gastroinestinal (colorectal, gastric, pancreatic cancer), lung, breast and gynecological cancers. In summary, hCG still has retained its mandatory role as essential clinical tumor marker for germ cell tumors and mainly as prognostic marker in many nontrophoblastic tumors. 


\title{
HCG IN DIAGNOSIS AND MANAGEMENT OF GESTATIONAL TROPHOBLASTIC DISEASE (GTD)
}

\author{
Michael J Seck1 \\ Charing Cross Hospital Gestational Trophoblastic Disease Centre, Imperial College London, Fulham Palace Rd, \\ London W68RF, UK
}

Gestational trophoblastic disease (GTD) comprises a spectrum of disorders from the pre-malignant complete (CM) and partial (PM) hydatidiform moles through to the malignant invasive mole, choriocarcinoma and rare placental site trophoblastic tumor (PSTT). All forms of the disease produce hCG which serves as a useful marker of GTD activity. The commonest GTD variants, CM and PM, affect between 1-3:1000 pregnancies and usually present with vaginal bleeding in early pregnancy. Following evacuation, all these patients require close monitoring for the development of malignant change which occurs in 16 and $0.5 \%$ of CM and PM, respectively. The simplest way to detect malignant change is by serial hCG monitoring. With benign disease, the hCG returns to normal but in those developing malignant change, the hormone plateaus or starts to rise with time. Such patients require chemotherapy to eliminate their disease. Regular measurements of hCG enables the clinician to accurately monitor the response to therapy with far greater sensitivity than any other imaging or biochemical modality. Moreover, the onset of resistance can be quickly detected enabling a rapid switch to more intensive treatments. Once the disease is in remission, continued hCG monitoring is the most sensitive way to detect recurrence. Thus, the accurate measurement of hCG is essential for detection of malignant change, monitoring of treatment response and subsequent surveillance for relapse in patients with GTD. Obviously, an understanding of the types of hCG produced in health and disease maybe important. Indeed, in pregnancy, hCG is either intact or hyperglycosylated but in cancer may be found in various additional forms. hCG assays used to monitor cancer patients should detect all circulating forms of $\beta \mathrm{hCG}$ and should not suffer from either false positive or negative results. The latter problem is not yet widely appreciated and will be discussed.

S4_A5

\section{VARIOUS ISOFORMS OF HCG IN CANCER PATIENTS}

Ulf-Håkan Stenman

Helsinki University Central Hospital, Biomedicum, FIN-00029 Helsinki, Finland

Human chorionic gonadotropin (hCG) is a heterodimeric glycoprotein hormone produced by cytotrophoblasts during pregnancy. Placental and other trophoblastic tumors virtually always express hCG, and for these hCG is a very sensitive marker. The pituitary also produces hCG and low levels occur in serum of healthy subjects. In postmenopausal women, levels up to 10 - $15 \mathrm{IU} / 1$ (30 - $45 \mathrm{pmol} / \mathrm{l})$ may occur and similar levels can be also be detected in males with suppressed gonadal function. The free beta subunit of hCG (hCG-beta) is produced at low concentrations by $20-50 \%$ of various non-trophoblastic tumors and this is a strong sign of adverse prognosis. Contrary to the concentrations of hCG, those of hCG-beta are not dependent on age and gonadal function and the upper reference limit is below 2 pmol/1. Thus assays specifically measuring hCG-beta detect tumors producing this form of hCG much earlier than "total hCG" assays that measure hCG and hCG-beta together. When such assays are used to measure hCG-immunoreactivity in serum, elevated levels are detected only occasionally in nontrophoblastic cancer. Specific measurement of hCG-beta is especially useful in patients with testicular cancer and in seminoma this increases the frequency of marker positivity from about $15 \%$ to $50 \%$. The carbohydrate structure of hCG is variable and that produced in early pregnancy is more heavily glycosylated than late pregnancy hCG. This so called hyperglycosylated hCG (hCG-h) is also produced by malignant trophoblasts. It can be detected by assays using the monoclonal antibody B152, but the clinical utility of such assays remains to be determined. 


\title{
THE TKTL1 GLUCOSE METABOLISM AND MITOCHONDRIA-INDEPENDENT ENERGY PRODUCTION IN CANCER CELLS
}

Jürgen Wilde ${ }^{1}$, Rainer Wittig ${ }^{1}$, Christoph Otto ${ }^{2}$, Nadja Pfetzer $^{3}$, Ulrike Kämmerer ${ }^{3}$, Peter Schubert ${ }^{1}$, Johannes F Coy ${ }^{1,4}$

R-Biopharm AG, Landwehrstraße 54, D-64293 Darmstadt, Germany (1); Experimental Transplantation Immunology, Department of Surgery, University of Würzburg Hospital, Oberdürrbacher Str. 6, D-97080 Würzburg, Germany (2); Department of Obstetrics and Gynecology, University of Würzburg Hospital, Josef-Schneider-Str. 2, D-97080 Würzburg, Germany (3); TAVARTIS GmbH, Kroetengasse 10, 64853 Otzberg, Germany (4)

Recent findings suggest a close relationship between common genetic alterations in tumor cells and the Warburg effect (aerobic glycolysis), which is characterized by a fermentation of glucose to lactate and a concomitant mitochondriaindependent energy production even in the presence of oxygen. The pentose phosphate pathway allows glucose conversion to ribose for nucleic acid synthesis and glucose degradation to lactate. The non-oxidative part of the pentose phosphate pathway is controlled by transketolase enzyme reactions. We have detected up-regulation of a mutated transketolase transcript (TKTL1) in human malignancies, whereas transketolase (TKT) and transketolase-like-2 (TKTL2) transcripts were not up-regulated. Strong TKTL1 protein expression was correlated to invasive tumors and to poor patient outcome. TKTL1 up-regulation in tumors allows a mitochondria-independent energy production and leads to enhanced, oxygenindependent glucose usage, and a lactate-based matrix degradation. Clinically the switch from a respiration-based energy production to a fermentation-based energy production is of utmost importance since this switch is often associated with resistance to chemo- and radiotherapy. Whereas most standard cancer therapies fail to inhibit fermenting tumor cells, inhibition of fermentation has been shown to efficiently block tumor proliferation and overcomes resistance to standard therapies. Since inhibition of TKTL1 transketolase enzyme by siRNA and by anti-TKTL1 compounds suppresses tumor growth and metastasis, TKTL1 represents a target for novel anti-cancer therapies. We suggest an individualized cancer therapy based on the determination of metabolic changes in tumors that might enable the targeted inhibition of tumor cells. A future anti-TKTL1-compound based cancer therapy may also be improved by a dietary change including a substrate limitation by a low glucose/low carbohydrate diet.

\section{S5_A2}

\section{PATHOLOGY AS THE BASIS FOR A NOVEL TARGETED THERAPY}

\author{
A. zur Hausen \\ University Hospital Breisacherstr. 115A, 79106 Freiburg, Germany
}

Malignant tissues ferment glucose to lactate even in the presence of oxygen (aerobic glycolysis; "Warburg effect"). The relevance of aerobic glycolysis for cancer cell biology is still controversially discussed. A mutated transketolase enzyme (transketolase-like-1/ TKTL1) has been suggested as an underlying effector of a mammalian glucose fermentation pathway in malignancies. Transketolase enzyme reactions enable oxygen independent glucose degradation and play a crucial role in nucleic acid ribose synthesis utilizing glucose carbons in tumor cells. Expression of the TKTL1 gene has been shown recently in diverse human malignancies. Analyses of the expression pattern and intensity of TKTL1 in human malignancies in relation to clinico-pathological parameters identify TKTL1 expression as a strong predictor of poor patient survival in colon and urothelial cancer. In addition, in a large patient cohort $(n=201)$ of non small lung cancers (NSLC) we have assessed the impact of TKTL1-expression by immunohistochmistry. Overexpression of TKTL1 in NSCLC was significantly associated with poor patient survival $(p=0.008)$. This prognostic effect remained significant in multivariate analyses $(p<0.0223)$. Since several studies recently have shown that inhibition of transketolase enzyme reactions effectively suppress tumor growth, TKTL1 represents a novel pharmacodiagnostic marker. Histopathological and immunohistochemical analyses represent the key diagnostic tool to evaluate possible anti-TKTL1 therapeutic strategies. In the present study we present the data of TKTL1 expression in NSLC and discuss its impact in relation to other TKTL1 expressing malignant tumors. According to our results TKTL1 harbors a high potential as a therapeutic target in cancer therapy. 


\title{
CLINICAL RELEVANCE OF TKTL1 EXPRESSION IN TUMORS
}

Ulrike Kämmerer ${ }^{1}$, Melanie Schmidt ${ }^{1}$, Mathias Krockenberger ${ }^{1}$, Niko Kohrenhagen ${ }^{1}$, Arnd Honig ${ }^{1}$, Johannes Dietl ${ }^{1}$, Johannes F Coy ${ }^{2}$

${ }^{1}$ Dpt. OB/Gyn, University of Wurzburg, Josef-Schneider Str. 4, D-97080 Wurzburg, Germany ${ }^{2}$ R-Biopharm AG, Landwehrstraße 54, D-64293 Darmstadt, Germany

Transketolase like-1 protein (TKTL1) is postulated to be the key enzyme of a recently described metabolic pathway that links the pentose phosphate (pathway) to the Embden-Meyerhof pathway. This pathway facilitates anaerobic glucose degradation. In 1924, Otto Warburg described a remarkable high rate of glycolysis even under aerobic conditions in a variety of tumor samples. This "aerobic glycolysis" seems to be a key feature of tumor cells and plays a role in tumorigenesis and resistance to standard radiation and chemotherapy. The "Achilles heel" of those glycolytic tumor cells is their dependence on high glucose supply for sufficient energy production.

Using immunohistochemistry, we detected TKTL1 to be highly associated with higher tumor grades and worse prognosis in all types of gynaecological cancers. Our findings reflect those found previously in different tumor types like urothelial and small cell lung cancer.

In order to use the TKTL1 positive tumor's addiction on glucose in a curative way, we started a clinical study reducing carbohydrates in patient'ss nutritition to a maximum of $70 \mathrm{~g}$ per day. Patients were selected according to the following criteria: advanced situation with detectable tumor mass, being positive for TKTL1, (rather) after conventional tumor therapy and willing and able to strictly follow the diet. All patients included in the study so far (with carcinoma of the endometrium, ovary, breast, stomach, pancreas and liver as well as with malign melanoma, granulosa cell tumor and glioblastoma) feel well under that type of nutritition, cachexia did not occur or could be reverted. Further, in some cases a clear inhibitory effect on tumor growth could be observed. In summary, detection of TKTL1 metabolism in tumor samples predicts poor outcome but offers the option of a special low carb diet stabilizing or improving the patient's situation and quality of life.

\section{S5_A4}

\section{METABOLIC GROWTH CONTROL OF HUMAN TKTL1-POSITIVE TUMORS IN NUDE MICE WITH A KETOGENIC DIET}

\author{
Christoph Otto $^{1}$, Ulrike Kämmerer ${ }^{2}$, Bertram Illert ${ }^{3}$, Bettina Mühling ${ }^{1}$, Arnulf Thiede ${ }^{3}$, Johannes F Coy ${ }^{4}$
}

Experimental Transplantation Immunology, Department of Surgery, University of Würzburg Hospital, Oberdürrbacher Str. 6, D-97080 Würzburg, Germany (1); Department of Obstetrics and Gynecology, University of Würzburg Hospital, Josef-Schneider-Str. 2, D-97080 Würzburg, Germany (2); Department of Surgery, University of Würzburg Hospital, Oberdürrbacher Str. 6, D-97080 Würzburg, Germany (3); TAVARTIS GmbH, Kroetengasse 10, 64853 Otzberg, Germany (4)

\begin{abstract}
Aims: One of the most prominent metabolic alterations in cancer cells is an increase of aerobic glycolysis which may provide potential targets for therapeutic strategies. Herein we describe the effect of a high fat/low carbohydrate diet (,,diet") on the growth of human cancer cells in nude mice. Methods: The diet delivers $15.4 \mathrm{~kJ} / \mathrm{g}$ gross energy, $2 \mathrm{~g} / \mathrm{Kg}$ carbohydrate, $355 \mathrm{~g} / \mathrm{Kg}$ fat, $130 \mathrm{~g} / \mathrm{Kg}$ protein. The standard feed (,,control“) delivers $12.8 \mathrm{~kJ} / \mathrm{g}$ gross energy, $364 \mathrm{~g} / \mathrm{Kg}$ carbohydrate, $70 \mathrm{~g} /$

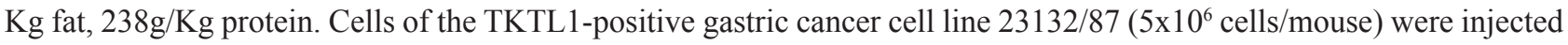
subcutaneously in both flanks of male NMRI ${ }^{\text {nude }}$ mice. Subsequently, the animals were randomized ( $\mathrm{n}=9$ per group) and received diet or control feed ad libitum. Results: The diet, well accepted by the mice, did not influence their body weight. The animals demonstrated a starting body weight of $29 \pm 1.8 \mathrm{~g}$ (finishing body weight: $28.9 \pm 1.8 \mathrm{~g}$ ) and animals of the control group weighed $29.6 \pm 1.1 \mathrm{~g}$ at the beginning and $29.5 \pm 0.9 \mathrm{~g}$ at the end. The diet did not influence the plasma glucose levels: $6.3 \pm 3.9 \mathrm{mmol} / 1$ (diet) versus $5.1 \pm 2.7 \mathrm{mmol} / 1$ (control). In contrast, the blood plasma levels of $\beta$-hydroxybutryrat were twice as high in the diet group $(1.3 \pm 0.5 \mathrm{mmol} / 1)$ as in the control group $(0.6 \pm 0.1 \mathrm{mmol} / 1)$. Tumors of the control group reached the tumortarget volume of $600 \mathrm{~mm}^{3}$ within $23.3 \pm 4.2$ days, whereas tumors of the diet group gained the tumortarget volume within 33.3 \pm 7.8 days. Conclusion: Feeding with the ketogenic diet delays the growth of human TKTL1-positive cancer cells in nude mice. The antitumor effect of the diet may be improved by simultaneous administration of specific TKTL1 inhibitors.
\end{abstract}




\title{
PLASMA PROTEOMICS - LOOKING FOR NEW BIOMARKERS
}

Gabriele Pestlin, Ingo Lindner, Stefan Palme, Michael Thierolf, Bernhard Risse, Werner Zolg

Roche Diagnostics GmbH, Roche Professional Diagnostics, Nonnenwald 2, D-82372 Penzberg, Germany

Proteomics biomarker discovery from human plasma holds both high clinical potential as well as significant challenges. The plasma proteome is generally accepted to be the most informative biological specimen as it contains thousands of proteins including proteins secreted or shed by cells as well as proteins that leak into the blood by damaged tissue. However, plasma proteomics is a challenge due to the wide dynamic protein concentration range with the highest complexity of any proteome. To overcome these hurdles and to extend the search of biomarkers into the relevant concentration range (ng/ml) directly in plasma, we developed a modular approach consisting of the depletion of 19 high abundant plasma proteins and the enrichment of low abundant proteins by chromatographic steps. After in-gel-digestion, the resulting peptides are separated by two-dimensional fractionation before being identified by mass spectrometry. With this approach we identified e.g. tumor markers such as CEA, differentially expressed in tumor plasma compared to control plasma, directly in plasma confirming that the methodology is suitable to directly mine the plasma proteome. In oncology, the plasma proteomics approach increases the likelihood of tracing relevant analytes directly in plasma, the compartment used to later follow disease related modulation of the markers by immunoassays.

\section{S6_A2}

\section{DISEASE SPECIFIC ALBUMIN PATTERNS DEFINED BY ELECTRON SPIN RESONANCE}

\author{
Vladimir Muravsky $^{1}$, Andrey Gurachevsky ${ }^{1}$, Gert Matthes ${ }^{1,2}$ \\ ${ }^{1}$ Medinnovation GmbH, Freiheitstrasse 124/126, 15745 Wildau, Germany \\ ${ }^{2}$ Institute for Transfusion Medicine, University Hospital, Delitzscher Straße 135, 04129 Leipzig, Germany
}

Aims:

Albumin conformation changes can be evaluated by spin probe ESR [1]. A variety of tumor-associated proteins and peptides released by tumor bind to albumin [2], leading to distinct structural and allosteric changes to this protein. Therefore, cancer induced albumin changes should be analyzed.

Method:

Albumin changes were assessed with use of 16-doxyl stearic acid spin probe. The test procedure requires $0.2 \mathrm{ml}$ of serum/ plasma and is completed within 20 minutes. Three $50 \mu \mathrm{l}$ aliquots of samples with different amounts of spin probe (5-10 $\mathrm{mmol} / \mathrm{L})$ and ethanol (10-14 $\mu \mathrm{l})$ are measured by ESR. The spectra are analyzed through simulation with least-square fitting of its components belonging to differently bound spin probes to calculate binding properties and structural changes of the albumin [3].

Results:

Studies including more than 2000 patients with different cancer types have demonstrated specific changes to albumin in cancer patients, which enables an early tumor diagnosis with approx. $95 \%$ specificity, and $90 \%$ sensitivity. Monitoring of patients who provided serial samples during cancer therapy showed a clear correlation between the extent of albumin modifications and the clinical outcome of patients.

Conclusions:

Changes to albumin may depend on the rate with which tumor cells release metabolites into circulation reflecting activity of the tumor. ESR spectroscopy of albumin patterns is a novel sensitive and noninvasive technique that demonstrates diagnostic utility in patients with cancer and enables monitoring of cancer in patients under therapy.

References:

1. Berliner LJ. Spin Labeling: Theory and Applications. New York: Academic Press; 1976.

2. Liotta LA, Petricoin EF. Serum peptidome for cancer detection: spinning biologic trash into diagnostic gold. J Clin Invest 2006 Jan;116(1):26-30.

3. Kazmierczak SC, Gurachevsky A, Matthes G, Muravsky V. Electron spin resonance spectroscopy of serum albumin: a novel new test for cancer diagnosis and monitoring. Clin Chem 2006 Nov;52(11):2129-34. 


\section{PATTERN RECOGNITION BIOMATHEMATICS FOR BIOMARKER DEVELOPMENT}

Stephen D. Barnhill, M.D.

Chairman and CEO, Health Discovery Corporation, 5501 1/2 Abercorn Street, Savannah, Georgia 31405, USA

Pattern Recognition has become the critical mathematical technique used to make sense of the vast amount of Omic data being generated today. It is also used for the accurate and automated interpretation of medical images from diagnostic radiology to anatomic pathology. Biomarker discovery from large amounts of data, whether generated from a clinical laboratory or diagnostic radiology center, require a thorough knowledge and understanding of pattern recognition techniques. The Support Vector Machine (SVM) has been demonstrated to be among the best pattern recognition techniques available for Biomarker Discovery. An understanding of SVM's as well as specific examples of their success in Cancer Biomarker Discovery will be presented. 


\title{
HER-2/NEU ONCOPROTEIN SERUM LEVELS IN PATIENTS WITH BREAST CANCER: COMPARISON WITH CEA AND CA 15.3
}

\author{
R.Molina, JM Auge, M. Muñoz, J. Pahisa, A. Torne, M. Velasco, B. Farrus, X. Filella \\ (Oncobiology Unit, Hospital Clinic,Medical School, Barcelona, Spain). \\ Oncobiology Unit, Hospital Clinic,Medical School, Hospital Clinic, Villarroel 170, Barcelona 08036, Spain.
}

The objective of this study was to evaluate the utility of serum HER-2/neu as tumor marker in patients with breast cancer. We studied:

1) 637 patients with untreated primary breast cancer. Abnormal Her-2 serum levels were found in 9,1\%, CEA in 12,4\% and CA 15.3 in $15 \%$ of the patients. Significantly higher C-erbB-2 levels were found in patients with HER-2 tissue overexpression than in those without it $(p<0,001)$. All tumor markers (Her-2 only in patients with positivity in tissue) were correlated with tumor size and nodal involvement and were prognostic factors with significantly shorter DFS and OS in patients with pretreatment tumor marker positivity.

2) 385 patients without evidence of residual diseases (NED). Her-2 was the first sign of recurrence in 26,5\% of the 107 patients with recurrence and in the $87 \%$ of those patients with overexpresion in tissue. The addition of Her- 2 to CEA and CA 15.3 increase the sensitivity in the early diagnosis in $11,2 \%$.

3) 520 patients with recurrence. Her- 2 was abnormal in $44 \%$, CEA in $59 \%$ and CA 15.3 in $76 \%$ of the studied patients. One or another tumor marker was positive in $90 \%$ of the patients. All tumor marker serum levels were correlated to the site of recurrence with the highest concentrations in patients with visceral metastases and the lowest in patients with loco-regional recurrences.

In summary, Her-2 is a useful tumor marker in patients with breast cancer, mainly as prognostic factor, in the early diagnosis of recurrence and in the follow-up of patients with breast cancer.

S7_A2

\section{PUNCTA, BREAST CANCER AND HER2/NEU. PROTOTYPE OF A COMPUTER SUPPORT FOR THE VISUALIZATION OF HEALTH CARE CONSUMPTION}

\author{
Marius Nap, Jeroen Donkers * and Eric Postma * \\ Dept of pathology, Atrium medical center, PO-Box 4446, Zip 6401 CX Heerlen, Nl and \\ * MICC, Maastricht university, The Netherlands.
}

Documentation and analysis of health care consumption is a laborious administrative task and it is even more difficult to take aspects of individual tumor characteristics and co-morbidity into account. We have developed a computer support system to visualize health care contacts of an individual with all available disciplines in the hospital. Every contact with the hospital is presented as an event with a colored dot in a 2 or 3 dimensional space, in relation to the time point of the first diagnosis and the medical specialty.

Based on the availability of data on Her2/Neu, we have selected 407 breast cancer patients, with 34731 events during 4 years of follow up. Her2/Neu over expression occurred in 66 patients with 5625 events. Intermediate Her2/Neu resulted in 187 patients with 16021 events and low or negative Her2/Neu showed 137 patients with 11110 events. Comparing events before and after the reference event of the first surgical contact confirmed expected contact profiles with internal medicine, laboratory medicine, nuclear medicine and radiology. No direct relation was seen between the level of Her2/ Neu and the pattern of health care consumption. For both cardiology and pulmonology the pattern of consumption did not change. Day care admissions were found shortly after the surgical event and at the end of the follow up period and they were associated with lower Her2/Neu scores. There seems to be no relation between the level of Her2/Neu expression and new cardiology contacts after the surgical event. Within Puncta, health care consumption profiles of hundreds of patients, having thousands of contact events can be visualized in a moment and this allows both doctors and hospital administrators to check for similarity of consumption with protocols, or to identify unexpected patterns and formulate new hypothesis that may lead to better planning of limited resources. 


\section{CLINICAL VALUE OF SERUM HER-2/NEU TESTING}

Lüftner D, Possinger K

Lüftner Diana, Charité Campus Mitte, Charitéplatz 1, 10117 Berlin, Germany

The human-epidermal-growth-factor receptor 2 proto-oncogene (HER2, neu, ErbB-2) is a transmembrane receptor which possesses an intracellular tyrosine kinase activity. It is overexpressed by immunohistochemisty (IHC) or amplified by fluorescence in situ hybridization (FISH) analysis in approximately 20 to $25 \%$ of invasive primary breast cancers and is associated with a poor prognosis and more aggressive disease. The HER-2/neu extracellular domain (ECD) is cleaved by the ADAM metalloproteinases, and the remaining membrane-bound internal domain is constitutively activated. The extracellular domain (p97-115 kD) of the HER2/neu protein is released into the circulation, and serum HER-2/neu levels are elevated in $30-70 \%$ of patients with metastatic breast cancer. Rising serum HER2/neu concentrations have been associated with progressive metastatic disease and poor response to chemotherapy. Furthermore, studies have shown that an elevated pretreatment HER-2/neu level is associated with decreased response to both first and second-line endocrine therapy. Studies have also shown that the rise and fall of serum HER-2/neu correlates with the clinical course of disease in $\mathrm{MBC}$ patients who have received trastuzumab and chemotherapy. Several studies have reported that a decline in serum HER-2/neu from pre-treatment to post-treatment levels predicted the clinical outcome from trastuzumab-based therapy.

S7_A4

\section{REASSESSMENT OF HER2 STATUS IN BREAST CANCER PATIENTS AT THE TIME OF METASTATIC DISEASE BY SERUM HER2 AND HER2 STATUS OF CIRCULATING TUMOR CELLS}

Tanja Fehm

University of Tuebingen, Calwer Str. 7, 72076 Tuebingen, Germany

Introduction: Several studies have indicated that HER2 status of breast cancer patients can change during the course of disease. Therefore, reassessment of the HER2 status at the time of disease progression might help to optimize treatment decisions. In this context, determination of serum HER2 or the evaluation of HER2 status in circulating tumor cells (CTCs) could be of relevance. Therefore, the aim of this study was to determine the serum HER2 status and the HER2 status of corresponding CTCs in metastatic breast cancer patients with initially HER2 negative or unknown HER2 status.

Methods: Blood samples were obtained from 77 metastatic breast cancer patients with negative $(n=44)$ or unknown $(\mathrm{n}=33)$ HER2 status. Serum HER2 was determined using the commercial HER-2/neu ELISA-kit (Oncogene Science, MA, USA). CTCs were detected by a slide based assay using immunomagnetic enrichment and characterized by phenoand genotyping. Alternatively, a commercial kit (Adnagen, Langenhagen, Germany) based on RT-PCR was used for CTC detection and characterization.

Results: Twenty of 77 metastatic patients had elevated serum HER 2 levels. Blood samples were analyzed for presence of CTC in 67 patients. 8 of 21 patients with CTCs showed HER2 amplification. Concordance between HER2 status of circulating tumor cells and serum HER2 was seen in 15 of 21 patients (71\%). In 6 patients conflicting results were obtained. 3 patients with elevated serum HER2 status had HER2 negative CTCs whereas three patients with HER2 amplified CTC had normal serum HER2 levels.

Conclusions: Our study confirms that a subset of HER2 negative patients develop elevated serum HER2 levels and HER2 positive CTCs associated with metastatic disease. This is clearly of clinical relevance since these patients in current practice do not have access to HER2 targeted therapy. 
THE ORIGIN OF CANCER DNA METHYLATION PROFILES

Peter W. Laird

Departments of Surgery and of Biochemistry and Molecular Biology; USC/Norris Comprehensive Cancer Center; 1441 Eastlake Ave., Room 6418; Los Angeles, CA 90089-9176; U S A

Epigenetic mechanisms are increasingly recognized as key contributors to cancer biology, diagnostics and therapeutics. For example, the discovery of epigenetic silencing of tumor-suppressor genes has expanded our understanding of Knudson's two-hit hypothesis in cancer ontogeny. Most studies have focused on the functional consequences of epigenetic events in cancer. However, it has become clear that epigenetic alterations occur in cancer-type and subtype specific combinations, often without an obvious underlying selective mechanism to explain many of the epigenetic hits. The identification of groups of cancer cases with similar epigenetic profiles can lead to the identification of groups of genes with similar epigenetic behavior. The shared epigenetic behavior among genes can lead to mechanistic models to explain the epigenetic profiles, while the shared epigenetic behavior among cancer cases can lead to the identification of novel subtypes of disease with distinct pathogenetic, clinical and biological features. We will present several examples of the identification and characterization of known and novel subgroups of human cancer.

S8_A2

EPIGENETIC SILENCING OF GENES INVOLVED IN SIGNALING PATHWAYS IN GASTRIC CANCER

Minoru Toyota, Hiromu Suzuki, Masanori Nojima, Hiroshi Yasui, Yasuhisa Shinomura, Kohzoh Imai

First Department of Internal Medicine, Sapporo Medical University, Sapporo 060-8543, Japan

Gastrointestinal cancer arises through accumulation of multiple genetic changes involved in cellular signaling pathways. Unlike colorectal cancer, frequencies of mutations in APC, K-ras and p53 are relatively low in gastric cancer, indicating the role of epigenetic changes such as DNA methylation in tumorigenesis of gastric cancer. To elucidate the role of DNA methylation of genes involved in WNT, Ras and p53 signaling pathway in gastric cancer, we examined aberrant methylation of genes involved these signaling pathways. Aberrant methylation of SFRP1, SFRP2, SFRP5, and DKK2 are frequently detected in gastric cancer. Restoration of these genes resulted in down-regulation of transcriptional activity of beta-cetenin/TCF4, indicating that epigenetic inactivation of these genes plays a role in altered WNT signaling pathway. We next examined methylation status of RASSF1-6 in gastric cancer, and found that RASSF2 is frequently silenced by DNA methylation. In silico analysis of genes inactivated by DNA methylation in cancer revealed that many genes have potential p53 response elements. Treatment of gastric cancer cell lines with 5-aza-dC enhanced adenoviral-mediated p53 target gene induction, indicating that epigenetic inactivation of genes regulating signaling pathway are necessary for development and progression of gastric cancer. Subsets of gastric cancers show simultaneous methylation of multiple genes, indicating that these tumors have $\mathrm{CpG}$ island methylator phenotype (CIMP). Gastric cancers with CIMP show distinct clinicopathological features, such as diffuse type histology, and Epstein-Barr virus infection. Dissection of the role of DNA methylation in gastric cancer may facilitate the diagnosis and treatment of the disease. 


\title{
ANALYTICAL AND CLINICAL FACTORS IN THE QUALITY AND STANDARDIZATION OF TWO PROSTATE CANCER TESTS
}

\author{
Harry Rittenhouse \\ Gen-Probe Incorporated, 10210 Genetic Center Drive, San Diego, CA 92121, USA
}

A primary goal of quality clinical laboratory tests is to develop assays that are analytically accurate, while maintaining the reported results consistent with historical clinical correlations. The serum PSA test is a good example of the difficulties to satisfy both parts of this goal. Historically the PSA "cut-off" has been reported as $4.0 \mathrm{ng} / \mathrm{mL}$. In the standardization process to normalize different PSA assays and report the absolute mass amount of PSA some discrepancy can occur between the analytical value reported for PSA in relation to the values associated with the earlier studies assessing the clinical "cut-off" for biopsy decisions. In addition, physicians monitoring individual patient's PSA values over time can be confused if assays have been changed to reflect analytical standardization.

The PCA3 molecular urine test has recently been introduced into several clinical laboratories. This presentation will focus on some of the issues to develop quality immunodiagnostic and molecular diagnostic tests using serum PSA and the urine PCA3 tests as models.

S9_A2

\section{HER2 GUIDELINE DEVELOPMENT TO IMPROVE TESTING QUALITY}

\author{
E Hammond
}

Dept of Pathology, LDS Hospital, Intermountain Healthcare, Salt Lake City, Utah 84103 USA

Trastuzumab has been shown to be an effective chemotherapeutic agent for both adjuvant and metastatic breast cancer treatment in HER2 positive patients on the basis of recent clinical trials in the USA and Europe. The same clinical trials highlighted the variation in results from HER2 testing, by either HER2 immunohistochemistry (variation average 18\%) or HER2 gene in situ hybridization (variation average 13\%).

Two USA professional organizations representing medical oncologists (ASCO) and pathologists (CAP) convened an international expert panel to define the issues and devise a guideline to address them. The panel defines the optimal testing algorithm and thresholds for positive, equivocal and negative results that relies on accurate, reproducible assay performance. Elements to reliably reduce assay variation (e.g., specimen handling, assay exclusion, and reporting criteria) are specified. Laboratories who desire to offer HER2 testing must show 95\% concordance with another validated test for positive and negative assay values. The panel strongly recommends validation of laboratory assay or modifications, use of standardized operating procedures, and compliance with new testing criteria to be monitored with the use of stringent laboratory accreditation standards, proficiency testing, and competency assessment. CAP has responded to the guideline development by producing mandatory proficiency testing materials and processes and by altering the laboratory inspection criteria for HER2 testing laboratories. The panel has addressed issues that have arisen after publication via written responses in journals and plans to update the guideline recommendations when new data is presented or published. CAP and ASCO will seek to have guideline recommendations widely disseminated to other accrediting agencies so that the requirements become generalized to all clinical laboratories in the USA. Laboratory performance will be monitored and reported to improve performance. This flexible, iterative process is hoped to impact on HER2 testing quality. 


\title{
ASSESSING QUALITY - AN EQA PERSPECTIVE
}

\author{
C Sturgeon
}

Dept of Clinical Biochemistry, Royal Infirmary of Edinburgh, Edinburgh EH16 4SA, UK.

The primary function of external quality assessment (EQA) is to provide each participating laboratory with an objective assessment of its own performance. Increasingly, however, data generated by EQA providers inform the strategic decisions of professional organizations, particularly those involved in developing guidelines for analytical performance. This places major responsibility on EQA providers to ensure that specimens distributed are appropriate, i.e. as similar as possible to patient specimens and of clinically relevant concentrations. The validity of target values (usually trimmed consensus means) requires regular confirmation, e.g. by assessing recovery of the relevant International Standard. Long-term assay stability is also particularly important for tumor markers.

Factors contributing to between-method differences include errors in calibration, differences in method specificity, and method design. Comparability of results has improved for some tumor markers: between-method coefficients of variation for PSA have decreased two-fold following almost universal adoption of International Standard 96/670 and highly commendable efforts by diagnostic companies to calibrate their PSA methods accurately. Significant methodrelated differences are still observed for many other tumor markers, particularly CA125 and the other CA antigens. Lack of International Standards for these markers limits progress in improving between-method comparability and should be addressed urgently. Antibody selection and method design is critical in order to achieve equimolarity of recognition of different isoforms (e.g. complexed and free PSA) and to minimize the risk of interference (e.g. heterophilic antibodies, "hooking").

EQA schemes can also undertake some assessment of activities in the pre- and post-analytical phases, through occasional surveys of practice relating to reference intervals, reporting procedures and clinical interpretation of results.

Much effort is being expended both nationally and internationally to improve the quality of tumor marker measurement. EQA provides a powerful tool with which to assess the success of these initiatives and to identify targets for further improvement.

\section{S9_A4}

\section{QUALITY REQUIREMENTS IN CLINICAL PRACTICE - A USER'S VIEW}

\section{Tholander Bengt}

\section{Gynecologic Oncolgy Section, Radiumhemmet, Karolinska University Hospital, SE 17176 STOCKHOLM, SWEDEN}

The clinical benefit of a diagnostic method is in the end depending on the consequences of the diagnosis, but is often evaluated by other criteria as; 1) Technical function, 2) Diagnostic accuracy, 3) Diagnostic importance, 4) Therapeutic importance and 5) Result for the patient. Additional criteria have been defined. An ideal tumor marker should be sensitive and specific. It should reflect tumor burden and dissemination. A useful tumor marker should also be prognostic of outcome and / or predict the result of specific treatment. It should preferably lead to more effective treatment for the patients. Unfortunately, no known tumor marker fulfils all these criteria. The evaluation is complicated since these indicators are used in many different clinical settings with heterogenous patient populations with respect to tumor biology and other characteristics. Different analytical methods and cut-off criterions are used. A large number of factors have impact on tumor marker results, which make interpretation complex. Based on evidence from published studies of the tumor marker CA-125 in the diagnosis and management of ovarian cancer, different quality aspects of tumor markers will be discussed in diagnosis, monitoring and follow-up as well as in clinical trials.

CONCLUSION. The value of CA-125 in serum for preoperative differential diagnosis of pelvic masses and for monitoring during treatment of ovarian cancer is established and this marker is recommended by the EGTM in this context. CA-125 is also recommended by GCIG, defining response and time of progression as endpoints in clinical ovarian cancer trials. In clinical practice only established evidence-based, diagnostic criteria for CA-125 should be used in the interpretation and this is true also for other markers and settings. For the clinician it is a challenge to assimilate and interpret tumor marker results with other clinical data, and to inform the patient in a balanced, appropriate way. 


\title{
PEPTIDE TRUNCATION BY DIPEPTIDYL PEPTIDASES : IMPORTANCE IN PHYSIOLOGICAL AND PATHOLOGICAL PROCESSES
}

Ingrid De Meester, Anne-Marie Lambeir, Simon Scharpé

Laboratory of Medical Biochemistry, University of Antwerp, Universiteitsplein 1, BE 2610 Antwerp, The Netherlands

In an era where several low-molecular weight dipeptidyl peptidase IV (DPPIV, CD26) and Fibroblast activation protein alfa (FAP, seprase) inhibitors are progressing along the line of preclinical and clinical development, researchers are overwhelmed with data on their effects in vitro and in vivo. This use of specific DPPIV inhibitors undoubtedly will also contribute to the understanding of the role of dipeptidyl peptidases in the in vivo truncation of various substrates. A state of the art on peptide truncation by different dipeptidyl peptidases and related peptidases including not only DPPIV, but also DPPII, DPP8, DPP9 and prolyl oligopeptidase will be given.

S10_A2

\section{DIPEPTIDYL PEPTIDASE IV (DPP-4) INHIBITION AS A TREATMENT FOR TYPE 2 DIABETES}

Nancy A. Thornberry

Merck Research Laboratories, R50G-2A-0201, Dept Metabol Disorders, P.O. Box 2000, E. Lincoln Avenue, Rahway NJ 07065, USA

Defective insulin secretion is a hallmark of Type 2 diabetes, and agents that increase the concentration of circulating insulin have proven beneficial in the treatment of diabetes. DPP-4 inhibitors are a new approach to diabetes that lower glucose, at least in part, via stabilization of the peptide hormone glucagon-like peptide-1 (GLP-1), which has a clearly established role in insulin biosynthesis and secretion. Sitagliptin (Januvia $\left.{ }^{\mathrm{TM}}\right)$ is a highly selective DPP-4 inhibitor that has been recently approved for the treatment of type 2 diabetes, representing the first new oral therapy for treatment of this disease since the introduction of troglitazone in 1998. Sitagliptin has been shown to produce meaningful glucose control in a range of type 2 diabetics without the many of the liabilities that are associated with other oral therapies, including weight gain, edema, GI intolerability, and hypoglycemia.

Although the role of DPP-4 in incretin regulation is well established, this enzyme has been implicated in many other processes, including immune function, chemokine processing, and regulation of neuropeptide and other glucagon family members. DPP-4 is a member of an emerging group of serine dipeptidases with specificity for cleavage after proline residues. Other members of this group include QPP, FAP, DPP8, DPP9, and PEP. Current understanding of the biology of DPP-4 and the potential importance of selective inhibition for safe and effective treatment of type 2 diabetes will be reviewed.

S10_A3

\section{DIPEPTIDYL PEPTIDASE IV AND RELATED MOLECULES: MARKERS AND GROWTH REGULATORS OF HUMAN GLIOMAS}

\author{
Busek $\mathrm{P}^{1}$, Stremenova $\mathbf{J}^{1}$, Krepela $\mathrm{E}^{1}$, Marek $\mathbf{J}^{3}$, Dbaly $\mathrm{V}^{3}$, Mares $\mathrm{V}^{2}$, Sedo A ${ }^{1}$ \\ ${ }^{1}$ Joint Laboratory of Cancer Cell Biology of the 1st Faculty of Medicine, Charles University in Prague, and the \\ ${ }^{2}$ Institute of Physiology, Academy of Sciences, Prague, Czech Republic, \\ ${ }^{3}$ Department of Neurosurgery and Pathology, Hospital Na Homolce, Prague, Czech Republic
}

Aim:

Altered dipeptidyl peptidase-IV (DPP-IV) like enzymatic activity, which can be attributed to canonical DPP-IV/CD26 and some other proteins including DPP-7, 8, 9, and seprase/fibroblast activation protein- $\alpha$, often accompany malignant transformation and tumor progression. A number of bioactive peptides such as substance $\mathrm{P}(\mathrm{SP})$ and chemokine stromal cell derived factor- $1 \alpha(\mathrm{SDF}-1 \alpha)$ are proteolytically regulated by removal of the N-terminal dipeptide by DPP-IV enzymatic activity. This study was set up to characterize alterations of DPP-IV-like enzymatic activity in human astrocytic tumors as well as the expression of receptors of some of its biologically active substrates implicated in gliomagenesis. Moreover, the role of DPP-IV in glioma cell growth and its regulations was assessed in vitro. 
Methods and Results:

Using real time RT-PCR, immunochemistry, histochemistry and enzymatic activity assays with selective inhibitors we observed significantly increased DPP-IV-like enzymatic activity in astrocytic brain tumors compared to control tissues. Non-malignant brain tissue contained DPP-IV-like enzymatic activity attributable mostly to DPP8/9, while the substantial part of the activity in gliomas was due to an increase of DPP-IV/CD26. A grade-related rise in expression of CXCR4, a SDF- $1 \alpha$ receptor, paralleled the rise in DPP-IV expression and activity.

Our in vitro studies however suggest that DPP-IV overexpression in DPP-IV transfectants is associated with cell growth arrest as demonstrated by growth curves and cell cycle analyses using flow cytometry. In addition, DPP-IV enzymatic activity could abrogate calcium signaling of a growth-promoting mediator SP.

Conclusions:

DPP-IV is upregulated in glioblastomas in vivo, although it exhibits rather an antiproliferative effect in vitro. Further studies are needed to elucidate possible modulation of the SDF-CXCR4 axis by DPP-IV in gliomas and to explain the seemingly contradictory role of DPP-IV on the level of transformed cell and tumor tissue.

This work was supported by IGA NR/8105-3 and MSMT 0021620808.

\title{
S10_A4
}

\section{SEPRASE IN THE PRE-METASTATIC NICHE AND TUMOR INTRAVASATION}

\author{
Wen-Tien Chen, Donghai Chen, Huan Dong, and Alanna Kennedy
}

Metastasis Research Laboratory, Department of Medicine, Stony Brook University, NY, United States 11794.

Aims:

Metastasis, the neoplastic process responsible for most cancer deaths, involves a highly complicated regulation of proteolytic enzymes in the tumor microenvironment some of which act on collagenous fibers and gelatin to allow tumor cells to metastasize. We have investigated if seprase / fibroblast activation protein-alpha (FAP- $\alpha$ ), a gelatin-degrading protease that is transiently expressed in malignant tumors, is responsible for the initiation of metastasis in a primary tumor.

Method:

To examine the requirement of seprase in the pre-metastatic niche that allows the tumor cell to enter the circulation and metastasize, we examined the tumor microenvironment and metastasis by fluoresacence imaging, immunohistochemistry and Western immunoblotting using antibodies directed against intact gelatin and its fragments.

Results:

LOX human melanoma cells transfected with shRNA to suppress the expression of endogenous seprase to different extents, when xenografted s.c. into SCID mice, were two to three times more likely to develop soft tumors consisting of blood infiltration and gelatin, and showed a 25-50-fold reduction of tumor intravasation and a 90-180-fold decrease in lung and liver metastases compared with mock-transfected LOX cells. Fold reduction of metastatic indicators correlated with the number of seprase-expressing cells in a tumor. To probe the functional role of seprase in initiating metastasis, peptides inhibiting seprase gelatinase activity from phage display peptide libraries, when applied in the xenograft, generated similar effects as the RNAi suppression of a malignant tumor, intravasation, and formation of lung and liver metastases. Also, similar results have been obtained from an experimental ovarian metastasis model using i.p. inoculation of SB247 human ovarian tumor cells in SCID mice.

Conclusions:

By targeting the gelatin-rich tumor microenvironment, seprase drives cellular invasion in the pre-metastatic niche. Because of its paucity in normal tissues, seprase can be a therapeutic protease target to confine a tumor at the primary site. 


\title{
CLUSTERING AND PROTEOLYTIC ENZYMES COMPLEXES FORMATION IN SPECIALIZED PLASMA MEMBRANE DOMAINS OF ENDOTHELIAL CELLS. ROLE IN ANGIOGENESIS.
}

\author{
Monica Salamone, Mario Nasca, Viviana Vesco, Maria Laura Giunta and Giulio Ghersi. \\ Dipartimento di Biologia Cellulare e dello Sviluppo, Università di Palermo, Viale delle Scienze, Edificio 16 - 90128 \\ Palermo -ITALY
}

Generation of functional vasculature is essential for growth and development of solid tumors. In addition, the vasal blood network is one of the preferential way used by tumor cells to escape primary tumor site and allow metastatic sites. To block angiogenesis appear to be an essential strategy to develop an efficient cancer therapy, and its comprehension could be a winner approach to generate specific therapy tools against solid tumor development.

When angiogenesis take place, both in physiological than in pathological conditions, several modification occur in endothelial cells (EC); in particular, to plasma membrane level redistribution of cell-cell/cell-substrate adhesion molecules and increase in proteolytic enzymes, including matrix metalloproteases (MMPs) and serine proteases, were observed. One of the preliminary process that take place when EC acquired angiogenic phenotype is to lost cadherins mediated cellcell contacts, inducing $\beta$-catenin translocation to the nucleus where, in association to TCF-4, can work as transcription factor. We have investigated using different approaches:

1- Computational, on the up-stream non translated sequences of several MMPs and SIMPs genes about the presence of the $\beta$-catenin/TCF-4 binding sequences hTBE-1 and hTBE-2;

2- Biochemical, on proteolytic enzymes mRNA (by rt-PCR), proteins and enzymatic activities (by immunoblotting and gelatin zymography) expression when cell-cell contacts were perturbed by addition of IgGs and/or Fabs recognizing cadherins extracellular (Cac-125) and cytoplasm (Pan-cadherin) domains;

if this could be the privileged way of angiogenic proteolytic enzymes expression.

Moreover, we investigated if proteolytic enzymes clustered in specialized plasma membrane protrusions and shed membrane vesicles of cells invading ECM. Clustered enzymes shown to degrade easily ECM components, and clustered enzymes the capability to generate permissive substrates to EC migration/invasion. Furthermore, ECM invasion from EC seems to be drive by transitory complexes association among proteolytic enzymes and cell adhesion molecules in specialized EC plasma membrane protrusions.

\section{S10_A6}

\section{NOVEL TARGETED THERAPIES IN LUNG CANCER}

\author{
$\underline{\text { Ravi Salgia }}$ \\ University of Chicago, Department of Medicine, Section of Hematology/Oncology Chicago, USA
}

There will be over 213,000 patients diagnosed with lung cancer and over 160,000 deaths from lung cancer in 2007 in the United States alone. Worldwide, there will be over 1.2 million patients with lung cancer, and the overall 5-year survival of $16 \%$. Lung cancer can be divided into non-small cell lung cancer (NSCLC) and small cell lung cancer (SCLC). Incidence of NSCLC is $84 \%$, with the common histologies of adenocarcinomas, squamous cell carcinomas, large cell carcinomas, and bronchioloalveolar carcinomas. The incidence of SCLC is $16 \%$, and over $2 / 3$ of these patients present with metastatic disease. In order to impact on the survival and curability of lung cancer, we must understand the basic biology of lung cancer and translate the findings into novel therapeutics. There are oncogenic abnormalities that have been described, such as Myc, Ras, EGFR, c-Met, that lead to transformation and survival of lung cancer cells. Also, lung cancer can have deletion of tumor suppression genes such as $\mathrm{p} 53, \mathrm{p} 16$, and/or Rb. There are a number of other pathways that have been activated or inactivated in lung cancer. As an example, the chemokine receptor CXCR4 is upregulated in SCLC and NSCLC, and also the ligand SDF-1a. These can be modulated by dipeptidyl peptidases such as DPP-IV and fibroblast activation protein (FAP). Previously, studies have reported that FAP was expressed in the stroma of lung tumor samples. Recently, this has been a target of inhibition in clinical trials. Discussed will be the role of chemokines and chemokine receptors in lung cancer, and their modulation. Also, the role of novel targeted therapies in lung cancer-especially as related to receptor tyrosine kinases such as c-Met and EGFR. 


\title{
IN VIVO IMAGING AND IN VITRO TUMORMARKER DETERMINATION IN CANCER OF THE THYROID, BREAST, AND SKIN
}

\author{
Peter Oehr ${ }^{1}$, \\ ${ }^{1}$ University of Bonn, Dept. of Nuclear Medicine, Germany
}

\begin{abstract}
Aim: Comparison of the diagnostic value of in vitro CEA, TPA, CA 15-3, CA19-9 to in vivo F-18-fludeoxyglucose (FDG) imaging by positron emission tomography (PET) in oncology.

Method: Investigations were made in 100 healthy individuals, 84 patients with benign diseases, and 274 cancer patients (cancer of the thyroid $(n=35)$, breast cancer $(n=48)$, lung cancer $(n=28)$, colorectal cancer $(n=65)$, and melanoma $(n=98)$ ). Tumor marker determination and FDG-imaging were done at the same day for each individual patient. In some cases, FDG-PET-imaging was only made because CT was negative. There was no FDG-PET imaging in healthy persons.

Results: Combined in vitro and in vivo cancer detection was 100\% complementary. In no case both, biomarkers and FDGPET, were negative in any individual cancer patient. Cases in which either only PET, or only biomarkers were positive, showed a superiority of FDG-PET imaging in all investigated tumor entities. In melanoma patients there were higher sensitivities for FDG-PET compared to S 100 for cancer activity stages A0 - A2. In case of stage A3 and during followup, however, S100 determinations were as useful as FDG-PET imaging. In patients with thyroid cancer, an elevation of thyreoglobulin (Tg) was generally followed at first choice by I-123 single photon emission tomography (SPECT) for detection of cancer lesions. However, in (non-medullary) thyroid cancer patients with increased Tg-serum levels and negative radioactive iodine, whole body scintigraphy by FDG-PET is indicated as second choice.

Conclusions: Relying only on in vitro biomarkers and omission of FDG-PET imaging will result in a much higher loss of cancer detection than doing it the other way round. Elevation of in vitro tumormarkers concentration is a strong indication for PET-imaging for localization of tumor lesions, improved staging and further treatment, especially when other imaging like CT had negative results.
\end{abstract}

S11_A2

\section{PET, CT AND TUMOR MARKERS IN LUNG CANCER}

\section{Kley K., Oehr P.}

Dept. Nuclear Medicine, University Düsseldorf and Jülich, Moorenstr. 5, 40225 Düsseldorf, Germany

Since its introduction in 1974, positron emission tomography (PET) has gained widespread use in diagnosis of cancer. At present, most publications deal with 18F-flurodeoxyglucose (FDG) as the PET tracer for detection of increased glucose uptake. This is also the case for non-small-cell lung-cancer (NSCLC). According to a meta-analysis, the sensitivity and specificity of FDG-PET in the diagnosis of pulmonary nodules is $96 \%$ and $78 \%$, respectively. As a result, there is now a consensus that FDG PET can improve diagnosis and staging in patients with NSCLC. On the other hand, there seems to be no literature comparing the synergy between PET and tumormarkers in this tumor entity. In order to find out whether there is a synergy between PET, computed tomography (CT) and tumor markers, we made a study on NSCLC patients $(\mathrm{n}=14)$ at the PET-Cyclotron Center Bonn, Germany. This study showed a positive- and a negative predictive value (PPV and NPV) of $81 \%$ and $80 \%$, respectively, for FDG-PET, compared to $73,7 \%$ and $71,4 \%$ for CT. Both imaging procedures combined had $100 \%$ sensivity and specificity, and the localization of PET-positive lymphnodes was improved by anatomic information based on CT. The relation between FDG- PET and the tumor markers CEA, TPA, NSE and SCC was investigated in a collective of 28 patients with lung cancer (26 with an NSCLC and 2 with small-cell-lung cancer). Both FDG-PET and CEA were positive in 43\%. In all other cases, FDG PET was true positive and CEA false negative. For TPA the results are similar with $48 \%$ both positives, and $52 \%$ only FDG PET positive. Evaluation of the interrelation of NSE and SCC with lung cancer (11 patients) resulted in a high PPV (both 100\%) but sensitivities were poor (38\% and $25 \%)$. 


\title{
S11_A3
}

\section{PET/CT AND PSA IN PROSTATE CANCER}

Kirsten Bouchelouche

PET \& Cyclotron Unit, Odense University Hospital. Smooth Muscle Research Center, Koege University Hospital. Denmark

In the United States prostate cancer is the most common cancer among men. The increased awareness of prostate cancer as a major cause of male cancer mortality has resulted in a challenge for tumor markers and imaging. It is important at primary diagnosis, follow-up and recurrence, to obtain accurate assessment of the disease stage in order to decide the most effective treatment. In recent years, positron emission tomography (PET) has undergone explosive growth. In prostate cancer PET has been one of the slowest areas to develop. This is mainly due to urinary excretion of PET tracers and variable tracer uptake in prostate cancer. The initial use of ${ }^{18} \mathrm{~F}$-FDG PET in prostate cancer was disappointing. However, several advances in PET/CT imaging of prostate cancer have been made the past years. New and more favorable PET tracers have already shown promising results, i.e., ${ }^{18} \mathrm{~F}$-choline, ${ }^{11} \mathrm{C}$-choline, ${ }^{11} \mathrm{C}$-acetate, and ${ }^{18} \mathrm{~F}$-flouride. The role of ${ }^{11} \mathrm{C}$-methionine, ${ }^{18} \mathrm{~F}$-FDHT (Fluorodihydrotestosterone) and anti- ${ }^{18} \mathrm{~F}-\mathrm{FACBC}$ (anti-1-amino-3- ${ }^{18} \mathrm{~F}$ fluorocyclobutanel-1carboxylic acid) remain to be elucidated further. The new radiotracers and further advancement in PET/CT techniques are expected to improve the performance of PET/CT in prostate cancer. Prostate specific antigen (PSA) is widely used as a tumor marker in prostate cancer. However, accumulating data demonstrate that there are limitations to the use of PSA as a screening test. Novel biomarkers that can differentiate benign from malignancy processes are needed. Recently, new biomarkers show promising results. The lecture will highlight the recent findings with regard to PET/CT and PSA in prostate cancer.

\section{S11_A4}

\section{PET/CT AND TUMOR MARKERS IN GYNECOLOGICAL CANCER}

\author{
Lubos Holubec, Ondrej Topolcan, Petra Mrazkova, Marketa Prazakova, Jan Zahlava and Jindrich Finek \\ Charles University, Medical School and Teaching Hospital Plzen, E.Benese 13, 305 99, Plzen Czech Republic.
}

Aims: The aim of the study was to compare diagnostic accuracy of the imaging methods (USG, CT, PET/CT) and tumor markers commonly used (CA 125) for primary diagnosing, therapy monitoring and also follow-up of ovarian carcinoma patients.

Method: 30 ovarian carcinoma patients, who underwent medical treatment at the Department of Oncology and Radiotherapy, LF UK in Plzen, were monitored. The authors assessed the contribution of diagnosing by imaging methods and routine monitoring of the tumor marker CA 125 in the patients. The authors also present a few case reports of diagnoses, treatment and follow up of patients with epithelial ovarian cancer. On the basis of these practical examples it is possible to make some general recommendations about interpretation of CA 125 results obtained in a "case-finding" setting in combination with PET/CT scanning.

Results: Diagnostic accuracy of imaging methods with tumor markers assessment was compared in 30 patients with diagnosed and treated ovarian cancer. Optimal correlation was achieved between the levels of CA 125 and PET/CT examination. The study presents several case reports when a PET/CT scan was ordered on the basis of tumor markers elevation during the follow-up. Only PET/CT findings correlated with the tumor markers elevation and in this way a relapse or progression of disease was detected. On the basis of combination of these methods it was possible to adjust or finish antitumor therapy in time.

Conclusion: Combination of modern imaging methods (PET/CT scan) with recommended tumor markers (CA 125) allows a prompt change of chemotherapy schedule depending on the effect of selected treatment. It also enables early detection of tumor progression during follow-up.

Acknowledgements.

This study was sponsored by the Research project MSM 0021620819. 


\section{S11_A5}

\section{FDG AND CC-PET AS A MODEL FOR TRACER AND DRUG MICRODOSING}

$\underline{\text { Peter Oehr }}^{1}$, Bjoern Eickmeier ${ }^{1}$, Kirsten Bouchelouche ${ }^{2}$

${ }^{1}$ University of Bonn, Dept. of Nuclear Medicine, Germany,

${ }^{2}$ University of Copenhagen, Koege Hospital, Denmark

Aims: Positron-emission-tomography (PET) and the tumor marker F-18 Fluoro-deoxy-d-glucose (F-18-FDG) are used for in vivo cancer detection in patients. We evaluated the potential FDG-PET using cell-cultures (CC) for microdosing. Microdosing is the application of drugs in a sub-pharmacological dose for early in vivo information about absorption, distribution, metabolisation und elimination of medical products.

Method: CC-PET including carrier free F-18-FDG, F-18-free FDG, and glucose.

Results: Phamacodynamics: Inhibition of cell proliferation was starting at 0,001 $\mathrm{mmol} / \mathrm{l} \mathrm{FDG}$ (equivalent to 182,15 $\mu \mathrm{g} / \mathrm{l})$, and the upper limit was found at $1 \mathrm{mmol} / \mathrm{l} \mathrm{FDG}$. There was no interaction between FDG und glucose $(\mathrm{p} \leq 0,05)$. Pharmacokinetics: Addition of (non-radiolabelled „carrier“)-FDG resulted in a significant inhibition of accumulation $(\mathrm{p} \leq 0,05)$ of intracellular F-18-activity, starting at $0,01 \mathrm{mmol} / \mathrm{l} \mathrm{FDG}$. At a concentration of $5 \mathrm{mmol} / \mathrm{FDG}$, there was a significant decrease $(p \leq 0,001)$ of F-18-activity in the cells. A dose-linearity for accumulation of F-18-FDG in U-251 cancer cells was found at concentrations from $0,01 \mathrm{mmol} / 1$ to $0,333 \mathrm{mmol} / 1 \mathrm{FDG}$. Removal of extracellular F-18-FDG from tissue culture medium did not accelerate the release of the tracer from the cells. It is concluded that elimination kinetics from the cells to the surroundings was independent of concentration effects.

Conclusions: Comparison of the lowest limiting FDG-concentrations found in CC-PET $(182,15 \mu \mathrm{g} / \mathrm{l})$ to the F-18-FDG concentrations used for detection of cancer cells in patients $(0,05-1 \mu \mathrm{g} / \mathrm{kg}$ FDG) show that those concentrations used in patients are more than a hundred times lower. The pharmacokinetic and pharmacodynamic results found by CC PET provided molecular insight into tracer-, carrier- and other molecular interactions and gave evidence that the application of F-18-FDG in patients can be regarded as safe. The study also demonstrates that CC-PET microdosing could be applied for the determination of concentration limits of new tracers or drugs for diagnosis or treatment. 


\section{DENDRITIC CELL-BASED VACCINES FOR CANCER: THE CURRENT STATUS AND FUTURE APPROACHES}

Theresa L. Whiteside

Hillman Cancer Center, 5117 Centre Ave., Suite 1.27, Pittsburgh, PA 15213, USA

Dendritic cells (DC) play a key role in inducing antigen-specific T-cell responses. Their use as immunologic adjuvants in active-specific immunotherapy of cancer has been widely adopted. Manufacturing of DC for therapy and ex vivo engineering of DC to optimize their clinical efficacy have been a major challenge, however. The ideal source of and type of DC, selection of best antigens and methods of loading as well as the optimal cocktail of cytokines for their maturation have yet to be defined. No potency assay for mature DC has been validated so far, and the most efficient route of immunization is not established. In addition, the optimal dose and clinical setting most appropriate for delivery of DC remain to be defined. While several clinical studies have demonstrated the generation of anti-tumor immune responses after DC administration, these immunologic responses only rarely correlate with clinical responses. The results of clinical vaccination trials using DC remain inconclusive, largely due to a lack of rigorous criteria for a large scale production, antigen loading, potency determination and delivery of these cells. Several different approaches to loading human DC and increasing their potency are available and their application in a carefully controlled setting should lead to greater clinical efficacy of DC-based anti-tumor vaccines. Improvements in the design of DC-based vaccine protocols and manufacture of DC products of consistently high quality are expected to result in substantially more frequent clinical responses in the future.

\section{S12_A2}

\section{MOLECULARLY DEFINED VACCINES FOR IMMUNOTHERAPY OF CANCER}

\section{Pedro Romero \\ Division of Clinical Onco-Immunology, Ludwig Institute for Cancer Research, Lausanne branch, Switzerland}

The molecular identification of $\mathrm{T}$ cell defined tumor antigens triggered the design of therapeutic vaccines targeting defined antigens. These new types of vaccines were tested in early phase clinical trials. To date, after approximately 15 years, well over 150 trials have been reported. While both vaccine formulation and monitoring of antigen specific $\mathrm{T}$ cell responses have steadily progressed, clinical impact of such vaccines remains relatively low. Indeed, the efficacy of vaccination in terms of elicitation of specific T cell immunity has reached $100 \%$ for certain formulations and is generally $40-80 \%$ in the majority of recently reported trials. In contrast, metanalysis of their clinical results found an objective clinical response rate of around 3\%. However, the incidence of favourable clinical outcomes associated to vaccination is estimated to be as high as $25 \%$. Moreover, induction of immunity correlates more often than not with favourable clinical course.

The Melan-A/MART-1 protein is expressed in melanocytes and the majority of primary and metastatic melanomas. An immunodominant HLA-A2 restricted epitope defined by the 26-35 peptide lends itself to detailed studies in both healthy individuals and metastic melanoma patients because of the unusually massive repertoire of $\mathrm{T}$ cell precursors available which are generated at high numbers in the thymus and maintained at high frequency in the peripheral immune system. A series of phase clinical studies performed in Lausanne have shown that it is possible to induce strong CD8 T cell responses in advanced melanoma patients when the antigenic peptide is injected subcutaneously as an emulsion in Montanide ISA51 together with a small amount of CpG-ODN, a class B TLR-9 agonist, at monthly intervals. Detailed analyses of the vaccine induced $\mathrm{T}$ cell response, by combined multiparameter flow cytometry and molecular approaches to gene expression and TCR repertoire, have allowed us to obtain a detailed picture of the response. I will discuss our recent results in terms of the dynamics and functional attributes of the vaccine modulated specific $\mathrm{T}$ cell response and of their implications for further vaccine optimization. 
S12_A3

\title{
IMMUNOSTIMULATORY/IMMUNOPROTECTIVE EFFECTS OF MACROMOLECULAR ANTICANCER THERAPEUTICS
}

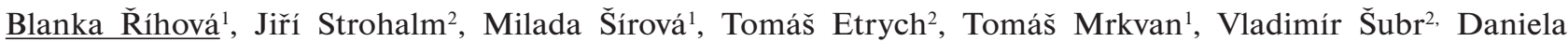 \\ Plocová2 ${ }^{2}$ Marek Kovár ${ }^{1}$, Karel Ulbrich ${ }^{2}$ \\ ${ }^{1}$ Institute of Microbiology, v.v.i., Academy of Sciences of the Czech Republic, Vídeňská 1083, 14220 Prague 4, Czech \\ Republic; ${ }^{2}$ Institute of Macromolecular Chemistry, v.v.i., Academy of Sciences of the Czech Republic, Heyrovsky Sq.2, \\ 16206 Prague 6, Czech Republic
}

Aims:

Cytotoxic and immunomobilizing activity of doxorubicin bound to water-soluble synthetic polymer based on $N$-(2-hydro xypropyk)methacrylamide (HPMA) was tested in vivo on mouse T cell lymphoma EL4. Both, non-targeted and antibodytargeted polymeric conjugates were used to compare their anti-tumor efficacy and the ability to induce specific or nonspecific anti-tumor resistance.

Methods:

Polymeric conjugates were synthesized as previously described ${ }^{1}$. Mouse $T$ cell lymphoma EL4 was purchased from ATCC and grown in a supplemented RPMI 1640 medium. In vivo experiments were done on conventional B6 and immunocompromised nude CD-1 mice ${ }^{2}$.

Results:

Doxorubicin was bound to the polymeric carrier either through an amidic (proteolytically cleavable) or hydrazone (hydrolytically cleavable, $\mathrm{pH}$ sensitive) bond. Both novel classes of anti-cancer therapeutics are highly efficient in killing tumor cells in vitro and are potent in eradicating growing tumors in vivo. In comparison with low-molecular-weight drugs, both types of macromolecular therapeutics, targeted or non-targeted, show decreased acute and delayed side-toxicity. More importantly, they trigger specific anti-tumor immune response. Such anti-tumor immunity can be transferred with splenocytes to naïve recipients. Thus chemotherapy based on conjugates of HPMA copolymer with doxorubicin could possesses immunomodulating properties.

Conclusions:

Polymeric conjugates of doxorubicin, non-targeted or antibody targeted cure mice from experimental EL4 T cell lymphoma and induce specific, systemic long-lasting anti-tumor resistance. 


\title{
LC-MS/MS PROCEDURE FOR MEASURING ZN-ALPHA2-GLYCOPROTEIN AS A TUMOR MARKER FOR PROSTATE CANCER
}

\author{
George G. Klee \\ Mayo Clinic, Stabile Building 2-44, 200 First Street SW, Rochester, MN 55905 U.S.A.
}

$\mathrm{Zn}-\alpha 2$-glycoprotein $(\mathrm{ZAG})$ is a protein with a molecular weight of $\sim 41,000 \mathrm{Da}$ that is overexpressed in certain malignant tumors and thus may serve as a potential cancer biomarker. However, ZAG is non specific and appears in multiple cancers (including prostate, bladder, and breast cancer), and patients with cachexia. ZAG circulates in relatively high amounts, therefore prior concentration is not needed for quantification by mass spectrometry. In this study we utilized trypsin mediated proteolysis combined with LC-MS/MS. We selected the ZAG tryptic peptide ${ }^{147}{ }^{17 P A W V P E D P A A Q I T K ~}{ }^{162}$ for quantification and used a stable isotope-labeled synthetic peptide with this sequence as an internal standard. Quantification was performed using an API 5000 triple quadrupole mass spectrometer (Applied Biosystems).

We used 3 pilot groups of men in this study. The 1st group included healthy men $(n=6)$, the 2nd group was men with nonmalignant prostate biopsy $(\mathrm{n}=20)$, and the 3rd group was men with $\mathrm{PCa}(\mathrm{n}=26)$. Precision studies using 2 serum pools run over 3 consecutive days showed $\mathrm{CVs}<7 \%$. The ZAG concentration from normal male sera gave an average concentration of $3.65 \mathrm{mg} / \mathrm{L}$. The average concentrations of ZAG in men with nonmalignant prostate disease and men with $\mathrm{PCa}$ were $6.21 \mathrm{mg} / \mathrm{L}$ and $7.59 \mathrm{mg} / \mathrm{L}$ respectively. The normals, non-malignant prostate samples, and samples from men with PCa showed statistical differences. Although ZAG is not a specific marker for prostate cancer, it may be a valuable biomarker to increase sensitivity for prostate cancer detection if it is combined with other more tissue specific biomarker markers, such as prostate specific antigen. This presentation is a summary and extension of the work recently published in Clin Chem 53:4, 673-678, 2007.

S13_A2

\section{MASS ANALYSIS TO DESCRIBE POST-TRANSLATIONAL CHANGES IN PROTEINS: LESSONS LEARNED FROM METABOLIC DISEASES}

\author{
John F. O’Brien, H. Robert Bergen III \\ Hilton Building $3^{\text {rd }}$ floor, Mayo Clinic, $2001^{\text {st }}$ Street SW, Rochester Minnesota 55905
}

Protein N-glycosylation is a fundamental biochemical pathway in eukaryotes which has been suggested to be a key process in development and cancer (1). The congenital disorders of glycosylation (CDGs) which limit N-glycosylation, result in multisystem disorders in childhood. The extent to which proteins are N-glycosylated (asparagine site occupancy) is determined by synthesis and transfer of a lipid-linked oligosaccharide in the endoplasmic reticulum.

Mass spectrometry (MS) is currently being used to determine the presence of $\mathrm{N}$-glycan structures in proteins (2). Because the absence of an N-linked oligosaccharide in CDG results in transferrin with a mass diminished by over 2200 mass units it is readily detectable using electrospray MS. With automation, it is possible to screen many samples in a day. Detection of CDGs is convenient because of a.) The abundance of transferrin in serum and b.) The loss of total oligosaccharides causing a large mass shift.

The question remains as to whether glycosylation variability occurs in neoplastic cells such that modification of glycosylation is significant enough to be seen in circulating glycoproteins or if the change in glycosylation will be limited to subtle microheterogeneity within the oligosaccharide. Such heterogeneity (fucosylation) has been implicated by the proponents of AFP-L3 which is being used as a risk marker for patients with chronic liver disease for progression to hepatocellular carcinoma (3). Questions remain relative to this markers sensitivity. We suggest that MS can be used to further evaluate the marker potential of AFP-L3 or other prospective glycoproteins which have been identified as products of neoplastic cells. 


\section{GENOMIC REARRANGEMENTS IN THYROID CANCER AND THE POTENTIAL ORGAN-STEM CELL ORIGIN OF THYROID NEOPLASIA}

$\underline{\text { Stefan K. G. Grebe }}$

Department of Laboratory Medicine and Pathology, Hilton 730C, Mayo Clinic, Rochester, Minnesota 55905, USA

Between 20-70\% of papillary and follicular thyroid carcinoma (PTC and FTC) exhibit recurrent disease-causing genetic rearrangements, a rate approaching that found in soft-tissue tumors or leukemias and exceeding $>10$-fold what is observed in other carcinomas.

Rearrangements are observed in $20-50 \%$ of PTC. They involve genes encoding for tyrosine kinase receptors that signal down the ERK pathway, including at least 12 different RET and 4 different NTRK oncogenic rearrangements, as well as at least one involving a downstream kinase, $B R A F$ (which might also be activated through mutations).

In FTC, $25-70 \%$ of tumors carry a $\mathrm{t}(2 ; 3 ;)(\mathrm{q} 13 ; \mathrm{p} 25)$ translocation that creates a fusion transcript consisting of the 5 '-region of the thyroid-specific transcription factor pax8 and the full coding sequence of the peroxisome proliferator-activated receptor gamma $(P P A R \gamma)$, a member of the super family of liganded thyroid hormone-steroid hormone nuclear receptors. The resultant fusion protein acts primarily through a dominant-negative effect on wild-type PPAR $\gamma$.

The trigger for the recurrent translocations in PTC and FTC is most likely DNA damage occurring in an organ stem cell. These are actively dividing cells with an active DNA repair systems and down-regulated apoptotic pathways, factors that favor recombination over cell death, in particular if the recombination event creates a further growth stimulus. The hypothesis of organ stem cell involvement is supported by observations in the Chernobyl population, where RET/PTC rearrangements have occurred in infants, suggesting a prenatal tumorigenic event.

In terms of clinical utility, the frequent occurrence of a limited number of well-characterized morphotype-specific tumor-genetic rearrangements affecting either ERK signaling (PTC) or PPAR $\gamma$ signaling (FTC) can be used to augment histopathological diagnosis, to monitor patients with high specificity for recurrent disease and to select individualized pathway-specific therapies, including selective kinase inhibitors (RET, NTRK, BRAF), or ligands for PPAR $\gamma$ or the retinoic X receptor (the obligatory PPAR $\gamma$ heterodimer partner).

\section{S13_A4}

\section{CIRCULATING ENDOTHELIAL CELLS AND PRECURSOR CELLS AS PREDICTORS OF CANCER SPREAD AND METASTASIS: PILOT DATA ON COLORECTAL AND BREAST CANCER}

\section{Lenka Dubska}

Department of Laboratory Medicine, Masaryk Memorial Cancer Institute, Brno, Czech Republic

Circulating endothelial cells (CECs) as well as their bone marrow-derived precursors (CEPs) play important role in tumor growth and tumor metastatic spread. The method for quantification of CECs and CEPs in peripheral blood using flow cytometry has been developed. We have detected CECs as non-hematopoetic (CD45-) expressing S-Endo (CD146+) in two major subsets - activated and resting CECs. Detection of circulating endothelial precursor has been based on their expression of endothelial and stem cell markers.

Detecting CECs in peripheral blood of patients with newly diagnosed carcinoma prior to therapy, we found significantly increased CEC number in patients with breast cancer compared to healthy donors. Moreover, we investigated whether the number of endothelial cells in the circulation correlates with soluble VEGF (vascular endothelial growth factor) in plasma. However, we found no correlation between CECs and VEGF levels nor CEPs and VEGF levels in newly diagnosed breast cancer patients.

VEGF blockade by bevacizumab has a benefit in response rate and overall survival when used in combination with existing metastatic colorectal cancer regimens. There is, however, a need for a marker of cancer angiogenesis for monitoring and prediction of response to the anti-angiogenic treatment exemplified by bevacizumab. Here we show our results from the study of CECs, CEPs and soluble angiogenic factors in patients with advanced colorectal cancer treated with becacizumab and chemotherapy.

This work was supported by the Czech Ministry of Health (Grant No. MZ00020980501). 


\section{OUTLINING PHARMACOGENETIC APPROACHES IN CANCER TREATMENT}

Dalibor Valik, Regina Demlova, Marketa Bednarikova,

Masaryk Memorial Cancer Institute, Zluty kopec 7, 65653 Brno, Czech Republic

Efficacy of anticancer treatment is governed by two fundamental principles: i) susceptibility of a tumor to an administered agent(s) and ii) capacity of the host to tolerate highly toxic treatment. Consequently, individualization of anticancer treatment can be approached from the standpoint of "predictive oncology" assessing tumor susceptibility and "pharmacogenetics" studying host response. Substantial effort has been recently invested in development of new agents selectively targeting various intracellular pathways important in tumor biology. However, the second decisive concept, pharmacogenetics, still remains clinically underexplored - though genetic factors undoubtedly play a significant role in overall tolerance of anticancer polychemotherapy, their study and quantification is often complicated by complex clinical variables such as overall comorbidity. Using metabolic and genetic biomarkers we have been studying three drugs important in chemotherapy of major cancers - methotrexate in pediatric ALL, 6-mercaptopurine in pediatric ALL and fluoropyrimidines in colorectal cancer. In pediatric ALL, we showed that plasma homocysteine is a functional biomarker of high-dose methotrexate efficacy and that pre-treatment plasma folate modulates its overall pharmacodynamic effect (Clin. Chem 2006, 52, 692-700); moreover, it has been shown recently that children receiving more leucovorine as a "rescue" agent after high-dose methotrexate have higher relapse rate (Leukemia 2006, 20, 1955-62). Here, we will present follow up studies of 5 versus $2 \mathrm{~g} / \mathrm{m}^{2}$ high-dose methotrexate regimens in ALL, pilot data on genotype/phenotype correlation in thiopurine methyltransferase and fluoropyrimidine degradation and outline of possible pharmacogenetic and biomarker-based approaches to study pharmacogenetic aspects of anticancer chemotherapy.

Contract Grant Number: MZ00020980501 


\title{
COMPUTER SUPPORTED RETRIEVAL OF INFORMATION NEEDS AND INFORMATION CONTENTS
}

\author{
Marius Nap, Loes Braun \\ Atrium medical center, Po box 4446, zip code 6401 CX, Heerlen, The Netherlands
}

In medical research, treatment and follow up, information retrieval is of vital importance. To retrieve the appropriate information, clinicians have to formulate clear information needs. These information needs can than be used as a starting point for information retrieval. However, physicians are not always aware of their exact information needs, and even if they are, they seldom have the time to perform adequate literature retrieval.

In our project, it is investigated to what extent a retrieval system incorporating the techniques as developed by Braun $e t$ al. can be used by the EGTM (European Group on Tumor Markers) in the field of tumor marker analysis. The purpose of the system is to (a) identify the exact information needs of the clinicians, and (b) provide information satisfying the identified information needs.

A prototype was developed with specific reference to cancer of the breast. The prototype contains general information about cancer, the TNM system, tumor markers in general, and evidence based medicine. The presented information is mainly obtained from the EGTM website. Furthermore, the prototype links to public websites for more general information and to PUBMED for more specific literature. Furthermore, the prototype contains a tutorial and a possibility to compose a specific patient case by combining various parameters including sex, age, tumor stage and grade and also comorbidity and therapy. Based on the defined patient cases, the prototype formulates information needs and retrieves literature automatically. The patient cases can be stored and used for teaching purposes to show how different compositions of parameters can lead to different formulations of information needs.

S14_A2

\section{TUMOR MARKERS IN CERVICAL CANCER FOCUSED ON THE USE OF SCC}

$\underline{\text { Katja Gaarenstroom }}^{1}$, Johannes Bonfrer ${ }^{2}$

${ }^{1}$ Department of Gynecology, Leiden University Medical Center, PO Box 9600, 2300 RC Leiden, The Netherlands

2 Department of Clinical Chemistry, Netherlands Cancer Institute, Plesmanlaan 121, 1066 CX, Amsterdam, The Netherlands

Although SCC is not suitable for screening or diagnosis of cervical cancer, serum SCC levels correlate with tumor stage, size, residual tumor after treatment, recurrent or progressive disease, and survival in patients with squamous cell cervical cancer. SCC is a sub-fraction of TA-4, a tumor-associated antigen and belongs to the family of serine protease inhibitors. SCC is not organ-specific or malignancy-specific. Serum SCC is elevated in $24-53 \%$ of patients with Stage IB or IIA squamous cell cervical cancer, and in 75-90\% of patients with advanced stage (FIGO IIB and higher) disease. Highly elevated pretreatment SCC levels may indicate the presence of lymph node metastases or extra-cervical spread, but a normal SCC level does not exclude the presence of lymph node metastases. In patients with Stage IB or IIA squamous cell cervical cancer, sensitivity of an elevated pre-treatment level of SCC to detect lymph node metastases has ranged from 60 to $87 \%$ with specificity ranging from 41 to $91 \%$.

An elevated pre-treatment SCC level has been found to be an independent risk factor for poor survival. It has been suggested that pre-treatment SCC level can identify patients who require intensive or additional treatment and may be of value in treatment planning. However, there is no evidence that more aggressive treatment improves pelvic control and survival in patients with elevated SCC levels.

SCC shows a strong correlation with the clinical course. Serum SCC level has a sensitivity between 56 and $86 \%$ and specificity between 83 and $100 \%$ for detecting recurrent squamous cell cervical cancer. Using SCC, a lead-time of up to 14 months for detecting recurrent disease has been reported, with a mean or median between 2 and 6 months. However, there is no evidence that earlier detection of recurrent disease using SCC monitoring influences treatment outcome or prognosis after primary treatment. 


\section{GUIDELINES FOR THE USE OF BIOMARKERS IN BREAST CANCER}

M J Duffy

Dept of Pathology and Laboratory Medicine, St Vincent's University Hospital, Dublin, Ireland

Practice guidelines are systematically developed statements to assist practitioners and patients in making decisions about appropriate health care for specific clinical circumstances (1). The aim of this presentation is to review existing guidelines on the use of biomarkers in breast cancer. The most widely used biomarkers in breast cancer are the estrogen receptor (ER), progesterone receptor (PR), and HER-2 which are measured in tumor tissue and CA 15-3, BR 27.29 (or other MUC1-related proteins), CEA, TPA and TPS which are measured in serum.

The American Society of Clinical Oncology (ASCO), National Academy of Clinical Biochemistry (NACB), National Cancer Center Network (NCCN), European Group on Tumor Markers (EGTM), European Society of Medical Oncology (ESMO) all recommend the measurement of ER, PR and HER-2 (2-5). In contrast to this consensus on tissue markers, different views exist on the use of serum markers in postoperative surveillance. Thus, while the EGTM recommend serial determination of CA 15-3 and CEA for the early detection of recurrent disease (3), ASCO (1996, 2001 and 2006), NACB, ESMO and NCCN are opposed to this practice $(2,4,5)$. According to ASCO, NACB and EGTM, serum markers such as CA 15-3 may be used in monitoring therapy in patients with advanced disease, especially in patients who have disease that is non-evaluable using radiological procedures. Currently, the use of newer tests such as those based on gene expression profiles or proteomics are not recommended for routine clinical use by any expert group.

1. Lyman et al, J Clin Oncol 2005;23:7703.

2. Khatcheressian JL, et al, J Clin Oncol 2006;24:5091-5097.

3. Molina R, et al, Tumor Biol 2005;26:281-93.

4. Pestalozzi BC, et al, Ann Oncol 2005;16 Suppl 1:i7-i9.

5. Duffy MJ et al, http://www.aacc.org/NR/rdonlyres/31054E0C-09B4-45FA-976C-FE26B78CD0F3/0/chp3f_breast.pdf

S14_A4

\section{A CRITIQUE OF THE AMERICAN(NACB) AND EUROPEAN (EGTM) GUIDELINES FOR THE USE OF TUMOR MARKERS IN DETECTION AND MONITORING CANCER}

\section{Fleisher,}

Memorial Sloan-Kettering Cancer Center, New York, N.Y., USA

In 2002 the NACB published Practice Guidelines and Recommendations for Use of Tumor Markers in the Clinic (Tumor Markers; Physiology, Pathology, Technology, and Clinical Applications, ed., E.P. Diamandis, et al. Chapter 5, 2002). In 2006, at a public forum as part of the scientific program at the AACC National meeting, the first draft of new and updated Guidelines on the Use of Tumor Marker in the Clinic, was presented and discussed. This draft is supported by evidence-based guidelines used to grade clinical utility of tumor markers in laboratory medicine. In this draft, the use of tumor markers in 16 malignancies, including breast, prostate, colorectal, lung, ovarian, and testicular cancers have been developed by sub-committees of internationally recognized experts in the field and their recommendations were available for comment on the NACB website. As is the case with most diagnostic tests, tumor markers are surrogate indicators of the presence of a cancer. Tumor markers have been used to screen for early cancer, establish a prognosis or monitor for disease recurrence or progression. The draft Practice Guidelines and Recommendations considered all aspect of tumor marker utilization. Optimally, a tumor marker result should separate patients receiving new, exploratory targeted therapy into populations whose outcomes reflect therapeutic efficacy with a clinical endpoint being that of survival.

This critic of the new NACB and EGTM Tumor Marker Guidelines will summarize the extensive review and recommendations of tumors markers from 16 malignancies. 
ANTIBODY-CYTOKINE COMPLEXES: MAGIC TOOLS FOR TUMOR IMMUNOTHERAPY?

Carsten Krieg, Sven Letourneau, Giuseppe Pantaleo, Onur Boyman

Division of Immunology and Allergy, University Hospital of Lausanne (CHUV), CH-1011 Lausanne, Switzerland

Cancers are among the leading causes of death worldwide, and current treatment options against most forms of cancer, especially for metastatic cancers, have been of limited success. Immunotherapy, in particular IL-2-based treatment modalities have shown some promise in metastatic renal cell carcinoma and metastatic melanoma. However, use of IL2-based immunotherapy has been hampered by considerable IL-2-induced adverse effects and high production costs. Moreover, some researchers are concerned about the fact that IL-2 is crucial for immune tolerance by maintaining T regulatory cells (Treg), which prevent immune responses against self-antigens, including tumor antigens. Hence, future cytokine-based tumor immunotherapy should reduce cytokine-induced adverse effects, be more cost-effective, and be selective in terms of boosting preferentially effector cells, such as T cells and natural killer (NK) cells. We have recently shown in mice that IL-2, when administered together with certain anti-IL-2 monoclonal antibodies (mAbs), causes dramatic proliferation of antigen-experienced (memory) CD8+ T cells and NK cells, increasing numbers of these cell subsets up to 100-fold [1]. Interestingly, such IL-2/anti-IL-2 mAb complexes are barely stimulatory for Treg. Moreover, increases in the biological activity of cytokines after association with mAbs apply to human IL-2 (plus anti-human IL-2 mAb), IL-3, IL-4, IL-6, and IL-7 (both for mouse and human IL-7) [2]. In conclusion, cytokine/mAb complexes might be a promising approach for tumor immunotherapy.

S15_A2 TRANSCRIPTIONAL REGULATION IN MELANOMA PROGRESSION: IMPLICATIONS FOR
THERAPY

Menashe Bar-Eli

Department of Cancer Biology, University of Texas M. D. Anderson Cancer Center, Houston, TX, USA 77030

The molecular changes associated with the transition of melanoma cells from radial growth phase (RGP) to vertical growth phase (VGP, metastatic phenotype) are not yet well defined. We have demonstrated that the progression of human melanoma is associated with loss of expression of the transcription factor AP-2. In metastatic melanoma cells, this loss resulted in overexpression of MCAM/MUC18 and MMP-2 and lack of c-KIT expression. In addition, inactivation of AP-2 in primary cutaneous melanoma cells by dominant-negative AP-2 (AP-2B) augmented their tumorigenicity in nude mice. We have also recently demonstrated that loss of AP-2 expression in metastatic melanoma cells resulted in over production of the thrombin receptor, PAR-1, which in turn, contributes to the metastatic phenotype of melanoma by upregulating the expression of adhesion molecules, proteases and angiogenic factors. Additionally, the transition of melanoma cells from RGP to VGP is associated with overexpression of the transcription factors CREB and ATF1, both of which may act as survival factors for human melanoma cells. Inactivation of CREB/ATF-1 activities in metastatic melanoma cells by dominant-negative CREB or by anti-ATF-1 single chain antibody fragment (ScFv), resulted in deregulation of MMP2 and MCAM/MUC18, increase the sensitivity of melanoma cells to apoptosis, and inhibition of their tumorigenicity and metastatic potential in vivo. In recent studies we have identified the platelet-activating factor (PAF) that is secreted by cells within the tumor microenvironment as a factor involved in phosphorylation and activation of CREB/ATF-1 in melanoma cells. In an attempt to inhibit tumor growth and metastasis of human melanoma in vivo, we have developed and generated two fully human antibodies targeting MCAM/MUC18 and IL-8. Their usage in preclinical studies will be discussed. 
THE ROLE OF THE IL-1 MOLECULES IN CARCIONGENSIS, TUMOR INVASIVENESS AND TUMOR-HOST INTERACTIONS

\author{
$\underline{\text { Ron N. Apte }}$ \\ Department of Microbiology and Immunology, Faculty of Health Sciences, Ben-Gurion University of the Negev, \\ Beer-Sheva 84105, Israel
}

Interleukin-1 (IL-1) is a pleiotropic pro-inflammatory and immunostimulatory cytokine with diverse effects on malignant processes. The IL-1 family consists of two agonistic proteins, namely IL-1 $\alpha$ and IL- $1 \beta$, and one antagonistic protein, the IL1 receptor antagonist (IL-1Ra), which binds to IL-1 receptors without transmitting an activation signal and thus represents a physiological inhibitor of pre-formed IL-1. In their recombinant form, IL- $1 \alpha$ and IL- $1 \beta$ exert the same biological activities and bind to the same receptors. However, in the physiological milieu, IL- $1 \alpha$ and IL-1 $\beta$ differ dramatically in the sub-cellular compartments in which they are active; IL-1 $\alpha$ is mainly active as a cell-associated cytokine (cytosolic and membrane-associated forms), while IL-1 $\beta$ is active only in its mature secreted form. As a ubiquitous mediator at the site of tumor development, IL-1 can be produced by microenvironmental cellular elements as well as by the malignant cells. Previously, we have shown, using fibrosarcoma cells transfected with the active forms of IL-1, that IL-1 $\alpha$ expression on the membrane of tumor cells increases their immunogenicity and leads to tumor eradication, while tumor cells which actively secrete IL-1 $\beta$ are of more malignant. To distinguish between tumor cell and host-derived IL-1, we used knockout (KO) mice that lack functional genes of members of the IL-1 family, i.e. IL-1 $\alpha$, IL-1 $\beta$, IL- $1 \alpha$ and IL-1 $\beta$ (double KO) and IL-1Ra KO mice as well as 3-methylcholanthrene (3-MCA)-induced tumors in control and IL-1 KO mice. The results indicate that microenvironmental IL-1 $\beta$ is essential for determining the malignant phenotype of transplantable tumors as well as for the induction of tumors following chemical carcinogenesis. We have also used fibrosarcoma cell lines obtained from control and the various IL-1 KO mice and the same set of recipient mice, to assess whether IL-1 of tumor cell- or host- origin are essential for invasiveness. The results indicate that IL-1 $\beta$ of both the malignant cell- and the hostorigin synergize in controlling invasiveness and metastasis of the tumor. Also, different forms of IL-1 that are expressed in the microenvironment of the host, where 3-MCA tumors develop, determine features of the malignant cells, such as invasiveness and immunogenicity. Altogether, the results point to differential effects of IL-1 $\beta$ and IL-1 $\alpha$ in malignant processes and points to the therapeutic feasibility of the IL-1Ra, which neutralizes soluble IL-1 (mainly IL-1 $\beta$ ), in tumor therapy, apart from its use in treatment of autoimmune diseases, such as Rheumatoid arthritis.

\title{
S15_A4
}

\section{CHEMOPREVENTION OF PROSTATE CANCER WITH VITAMIN D}

\author{
Barbara A. Foster \\ Roswell Park Cancer Institute, Buffalo, NY USA
}

Vitamin $\mathrm{D}$ has been investigated as a potential preventive therapy for prostate cancer $(\mathrm{CaP})$, because of its many anticancer activities. Vitamin D can inhibit cell growth and angiogenesis, as well as promote cell differentiation and apoptosis. Low circulating vitamin D levels correlate with increased risk for CaP. A transgenic mouse model of CaP (TRAMP) has been used to investigate the molecular mechanism of vitamin D's chemopreventive effects and the mechanism of escape from vitamin D's activity that results in vitamin $\mathrm{D}$ insensitivity in CaP. Calcitriol $\left(1,25(\mathrm{OH})_{2} \mathrm{D}_{3}\right)$ is the active metabolite of vitamin $\mathrm{D}_{3}$. The chemopreventive effects of $1,25(\mathrm{OH})_{2} \mathrm{D}_{3}$ and a less-calcemic vitamin $\mathrm{D}$ analog, $\mathrm{QW}-1624 \mathrm{~F}_{2}-2(\mathrm{QW})$, were tested in vivo using the TRAMP model. The TRAMP model was developed using the minimal probasin promoter to drive the expression of SV 40 early genes (T, t) specifically in prostatic epithelium ${ }^{1}$. The transgene (Tag) is expressed in prostatic epithelial cells at sexual maturity. Tag abrogates $\mathrm{p} 53$ and $\mathrm{Rb}$ function leading to deregulation of cell cycle and genomic instability. However, Tag expression alone is not sufficient to cause transformation and tumors. Other "hits" must occur ${ }^{2}$. As a result, with time TRAMP mice develop progressive stages of prostatic disease from early lesions of intraepithelial hyperplasia (PIN) to late stage metastatic cancer ${ }^{3-5}$. As seen in human CaP, TRAMP animals develop androgen-independent $\mathrm{CaP}$ following castration ${ }^{4}$. Approximately $80 \%$ of TRAMP animals castrated at 12 weeks-of-age have evidence of androgen-independent disease at 24 weeks-of-age ${ }^{6}$. The remaining $20 \%$ have no evidence of bulky disease and appear to be "cured", however histological evaluation indicates microscopic cancer is present. Of those animals that developed hormone refractory tumors, $100 \%$ had late-stage, poorly-differentiated grade 6 tumors, compared to only $33 \%$ of age matched intact TRAMP animals. Moreover, the incidence of metastasis was greater in the castrated 
cohort than the age matched intact cohort ${ }^{4}$. From these studies it appears that if castration does not "cure" the TRAMP animal, then the animal is more likely to present with late-stage, poorly differentiated CaP with metastases.

Vehicle, calcitriol $(20 \mu \mathrm{g} / \mathrm{kg})$ or QW $(50 \mu \mathrm{g} / \mathrm{kg})$ was administered prior to cancer initiate at 4 week-old TRAMP mice i.p. $3 \times /$ week for 14 weeks. $1,25(\mathrm{OH})_{2} \mathrm{D}_{3}$ and QW exhibited chemopreventive activity against $\mathrm{CaP}$ as indicated by reduced urogenital (UG) tract $(p<0.02)$ and prostate $(p<0.02)$ weights. However, only $1,25(\mathrm{OH})_{2} \mathrm{D}_{3}$ was able to maintain the expression of the pro-differentiation marker, E-Cadherin $(p<0.01)$, and reduce proliferation $(p<0.05)$ in the prostate. Chemopreventive effects of vitamin D compounds on development of hormone refractory $\mathrm{CaP}$ were tested in castrated TRAMP mice. Administration of $1,25(\mathrm{OH})_{2} \mathrm{D}_{3}(20 \mu \mathrm{g} / \mathrm{kg})$ or QW $(50 \mu \mathrm{g} / \mathrm{kg})$ to TRAMP mice either immediately after castration at 12 weeks-of-age or $1,25(\mathrm{OH})_{2} \mathrm{D}_{3}$ initiated at 4 weeks of age with castration at 12 weeks of age did not impact the formation of hormone refractory disease.

TRAMP animals were treated with either vehicle (Naïve, $n=15)$ or $1,25(\mathrm{OH})_{2} \mathrm{D}_{3}(20 \mathrm{ug} / \mathrm{kg}, \mathrm{n}=15)$ MWF starting at 4 weeks-of-age until palpable tumors developed. The rational was that continuous treatment of TRAMP animals with $1,25(\mathrm{OH})_{2} \mathrm{D}_{3}$ would select in vivo for vitamin $\mathrm{D}$ insensitivity (VDI). Understanding the mechanism of escape for VDI cells would identify key molecular targets for vitamin D's preventive action. In this experiment the median time to palpable tumor for naïve was 198 days (28 weeks) and for VDI 247 days ( 35 weeks). Histopathology of naïve and VDI tumors were indistinguishable with both being poorly-differentiated grade 6 tumors. Primary cell cultures were established from the tumors treated with vehicle alone (naïve, $\mathrm{n}=10$ ) or $1,25(\mathrm{OH})_{2} \mathrm{D}_{3}$ treated animals (VDI, $\mathrm{n}=8$ ). Characterization was performed on naïve and VDI cell cultures with a more indepth analysis performed on the most responsive naïve and least responsive VDI. Naïve and VDI cultures are composed of cytokeratin positive cells indicating they are epithelial in origin. Naïve cells are growth inhibited when treated with $1,25(\mathrm{OH})_{2} \mathrm{D}_{3}$ while VDI cells are not. Treatment with $1,25(\mathrm{OH})_{2} \mathrm{D}_{3}$ decreased proliferation as measured by BrdU incorporation of naïve cells and had no effect on VDI cells. There was no effect on apoptosis in either cell type as measured by Annexin staining. The VDR signaling pathway was investigated. $1,25(\mathrm{OH})_{2} \mathrm{D}_{3}$ treatment increased VDR expression in both cell types. However, when VDR mediated transcription was measured using the Cyp24-Luc reporter construct only naïve cells demonstrated appreciable levels of $1,25(\mathrm{OH})_{2} \mathrm{D}_{3}$ induced transcription. Naïve cells had 80-200 fold vitamin D-induced Cyp24-Luc activity compared to less than cyp24 gene expression in naïve cells and modest induction of expression in VDI cells as measured by qRT-PCR. The VDR was investigated as the explanation for reduced VDR mediated transcription. Exogenous VDR was not able to restore VDR mediated gene transcription in VDI cells. The VDR from the two least responsive VDI cells was sequenced and no mutations were identified. With $1,25(\mathrm{OH})_{2} \mathrm{D}_{3}$ treatment the VDR from naïve and VDI cells binds DNA and accumulates in the nucleus. One mechanism of vitamin $\mathrm{D}$ insensitivity that has been proposed is the increased catabolism of $1,25(\mathrm{OH})_{2} \mathrm{D}_{3}$ by increased activity of Cyp24 activity ${ }^{7-10}$. Therefore we examined Cyp24 protein levels and the effect of inhibiting Cyp24 activity with ketoconazol. If Cyp24 was mediating the insensitivity observed in VDI cells, we expect that VDI cells to express higher levels of the Cyp24 protein and activity and inhibition of cyp24 activity would restore vitamin D responsiveness in the VDI cells. However, Cyp24 protein is expressed at higher levels in the naïve cells with no differences in Cyp24 activity in naïve versus VDI cells. Inhibition of Cyp24 activity with ketaconazol did not sensitize VDI cells to the growth inhibitory effects of $1,25(\mathrm{OH})_{2} \mathrm{D}_{3}$, indicating that cyp24 activity is not a major mediator of vitamin D insensitivity in these cells. Gene expression profile was analyzed using Affymetrix GeneChip mouse genome 4392.0 Array comparing naïve-4 and VDI-1 cells $+/-1,25(\mathrm{OH})_{2} \mathrm{D}_{3}$. The gene expression profile also showed a global suppression of VDR-mediated gene transcription in VDI cells. Naïve cells had 294 genes that demonstrate a 2 -fold or greater change in expression. In contrast only 28 genes changed by 2 -fold or greater in VDI cells. Mining of the microarray data has identified differences in the expression of coregulator genes between unstimulated naive and VDI cells. Naïve cells express higher levels of the coactivators SRC-2, SRC-3, Hr, SKIP while VDI cells express higher levels of the corepressors $\mathrm{Hr}$ and LCor. We propose that the differences observed in naïve and VDI cells may be cell context specific such that differences in the coregulator profile of naïve and VDI cells account for the insensitive phenotype. 


\title{
REGULATION OF MTS GENE EXPRESSION
}

Paola Irato

Department of Biology, University of Padova, via U. Bassi, 58/B, 35131 Padova, Italy

Metallothioneins (MTs) play a role in the detoxification of heavy metals and the distribution of essential metals, and act as scavengers for reactive oxygen species (ROS). So their production can be induced not only by heavy metals but also by oxidative stress and a particular metal could enhance the expression of a specific isoform of MT. The regulation of MT gene expression is found to be highly complex: a variety of regulatory elements within MT promoter regions are identified. Four major MT isoforms, MT1, MT2, MT3, and MT4, have been identified in mammals. MT1 and MT2 isoforms are thought to be ubiquitously expressed, with MT3 and MT4 only selectively expressed in neurons and squamous epithelial cells respectively. MT1 and MT2 induction depends on the metal-regulatory transcription factor (MTF-1), which is a multiple Zn finger protein and the only known mediator of the metal responsiveness of MT1/2. Administration of essential or toxic metals increases MT biosynthesis by inducing their transcription, for which several cis-acting DNA elements, metal response elements (MREs) in the promoter region are binding sites for trans-acting transcription factors. The MT1/2 gene transcription is initiated when metals occupy the MRE-binding MTF-1. ROS also increase the MT1/2 transcriptional response, which rapidly increase MT1 mRNA levels in a dose-dependent manner. This induction could be related to presence of antioxidant responsive elements (ARE) positioned near the MRE, which could respond to ROS more that a direct action of metal on MRE. Different results suggest that MT1 isoform may respond to metal-induced ROS more than MT2 isoform. Our studies show that Fe, which is categorized as indirect MT inducer, causes an increase of MT1 expression. Moreover we observed that a fibroblast cell line MTF-1 mutant (knock-out) expressed MT1 isoform following metal treatment, but not the MT2 isoform.

S16_A2

\section{INTERPRETATION OF NATURAL FLUCTUATIONS OF METALLOTHIONEINS AND METAL LEVELS IN THE AQUATIC ORGANISMS}

\author{
Biserka Raspor \\ Rudjer Bošković Institute, Division for Marine and Environmental Research, 10000 Zagreb, Croatia
}

Metallothionein (MT) is established as a biomarker of metal exposure of aquatic organisms. In different tissue types, like gills, liver, kidney, intestine, of the aquatic organisms the levels of MT are related to the cytosolic metal concentrations $(\mathrm{Zn}, \mathrm{Cu}, \mathrm{Cd})$ that induce the MT synthesis.

Exposure of the aquatic organisms (shellfish and fish) under the controlled conditions clearly indicates de nuovo MT synthesis, but those experiments are performed at elevated metal levels that are usually not relevant for environmental conditions. In the field studies, fluctuations of different magnitude are observed for MT and cytosolic metal concentrations. They reflect individual response of the organism to different abiotic and biotic conditions, e.g. food availability, age, reproductive cycle etc. Therefore, within the overall MT concentration at the cellular level, the contribution of natural (biotic and abiotic) parameters to the basal level of MT and cytosolic metals, has to be defined before interpreting the results as being affected by metal pollution. Field studies conducted on indigenous fish and shellfish will be illustrated and discussed with respect to the limitations of using MT as the biomarker of metal pollution, in the indigenous aquatic organisms.

\section{References:}

V. Filipović Marijić and B. Raspor (2007) Metal exposure assessment in native fish, Mullus barbatus L., from the Eastern Adriatic Sea, Toxicology Letters: 168(292-301).

B. Raspor, Z. Dragun, M. Erk, D. Ivanković and J. Pavičić (2004) Is the digestive gland of Mytilus galloprovincialis a tissue of choice for estimating cadmium exposure by means of metallothioneins?, Science of the Total Environment: 333(99-108). 


\title{
COUPLING TECHNIQUES FOR METALLOTHIONEIN ANALYSIS AND CHARACTERIZATION
}

Dirk Schaumlöffel, Joanna Szpunar

CNRS, Laboratoire de Chimie Analytique Bio-Inorganique et Environnement, UMR 5254, Hélioparc, 2, Avenue du Président Angot, 64053 Pau, France

\begin{abstract}
Aims and methods:
The identification of metalloproteins at trace levels in a complex biological matrix is a great challenge for the analytical chemist. The key difficulties for the analysis and characterization of metallothioneins (MTs) in biological samples can be summarized as: (i) selectivity with regard to the different MT isoforms, (ii) selectivity with regard to metals, and (iii) sensitivity to cope with non-induced MT levels in biological tissues. These problems can be successfully addressed by coupling techniques based on the combination of a chromatographic separation with the parallel element specific (ICP MS) and molecule specific (ES MS/MS) detection. This pair of coupling techniques has the potential to be an interesting alternative to conventional biochemical methods for the characterization and quantification of metallothionein isoforms.

Results:

The lecture discusses the current development status of multidimensional coupling techniques based on the combination of HPLC (size exclusion and reversed phase) and/or capillary zone electrophoresis with ICP MS and electrospray MS for MT analysis and characterization. A new development will be presented, which is combining on-line isotope dilution with the coupling of capillary zone electrophoresis (CZE) to ICP-sector field MS. This method enables the quantification as well as the determination of the stoichiometrical composition of metal-metallothionein complexes by the quantification of the sulphur from the cystein residues and the metals: $\mathrm{Cu}, \mathrm{Zn}$ and $\mathrm{Cd}$.

\section{Conclusions:}

Three applications discussed for illustration concern the characterization of (i) rabbit liver MT and (ii) rat liver MT, which are bio-induced as response to cadmium stress, and (iii) the analysis of non-induced MT-3 in human brain, a case study in the context of Alzheimer disease.
\end{abstract}

\section{S16_A4}

\section{METALLOTHIONEINS AS A NEW POTENTIAL TUMOR MARKER}

Vojtech Adam $^{1}$, Sona Krizkova ${ }^{1}$, Ivo Fabrik ${ }^{1,2}$, Ondrej Zitka ${ }^{1,2}$, Zuzana Horakova ${ }^{3}$, Hana Binkova ${ }^{3}$, Jan Hrabeta ${ }^{4}$, Tomas Eckschlager ${ }^{4}$, Jiri Kukacka ${ }^{5}$, Richard Prusa ${ }^{5}$, Eva Sykorova ${ }^{6}$, Rene Kizek ${ }^{1}$

${ }^{1}$ Department of Chemistry and Biochemistry, Mendel University of Agriculture and Forestry, Brno, Czech Republic

${ }^{2}$ Department of Biochemistry, Faculty of Science, Masaryk University, Brno, Czech Republic

${ }^{3}$ Department of Otolaryngology and Maxillofacial Surgery, University Hospital, Brno, Czech Republic

${ }^{4}$ Department of Paediatric Haematology and Oncology, and ${ }^{5}$ Department of Clinical Biochemistry and

Pathobiochemistry, $2^{\text {nd }}$ Faculty of Medicine, Charles University, Prague, Czech Republic

${ }^{6}$ Institute of Sport Medicine, Brno, Czech Republic

Metallothioneins (MTs) are group of cysteine-rich metal-binding proteins. They play a protective role in heavy-metal poisoning and also maintain the metabolism of trace elements. Although MTs participate in the carcinogenic processes, their use as a predictive marker remains controversial. The present work reports on the involvment of MTs in cancer treatment, as a cause of resistance and in early detection of the disease as a potential new tumor marker. For determination of MTs in biological samples differential pulse voltammetry - Brdicka reaction was utilized. The involvement in resistance processes was investigated using a neuroblastoma cell line. In the cells resistant to cisplatin, maintained in medium containing cisplatin or carboplatin, the MTs levels were increased while in a sensitive cell line neither cisplatin nor carboplatin induced any significant MTs increase. To estimate the role of MTs as a new tumor diseases markers, a set of seventy patients with various tumor diseases (e.g. malignant melanoma, breast, colon and lung cancer) were analysed. The results obtained showed the average content of MTs determined in the blood serum of the cancer patients was around $2 \mu \mathrm{M}$, which is two times higher than that determined in the controls $(0.9 \mu \mathrm{M})$. These studies have been continued with specific attention on the analysis of blood and tumors from patients with tumor head and neck cancer. To date we have analyzed more than 250 blood samples and more than 60 tumor tissues. Based on these results, we propose that MTs could be used as predictive marker for head and neck cancer.

Acknowledgment: This work was supported by grants from Beh Terryho Foxe 2007, GAAV IAA 401990701, Liga proti rakovine and VUP 2007. 


\section{SERUM LEVELS OF METALLOTHIONEIN IN EXPANSIVE CONTUSIONS, INTERLEUKINE-6, AND TNF- DURING REPARATIVE PHASE OF THE BLOOD BRAIN BARRIER DAMAGE}

David Vaitr ${ }^{1}$, Jiri Kukacka ${ }^{1}$, Vojtech Adam², Karel Kotaska ${ }^{1}$, Ladislav Houstava ${ }^{3}$, Pavel Toupalik ${ }^{1}$, Rene Kizek ${ }^{2}$ and Richard Prusa ${ }^{1}$

${ }^{1}$ Department of Clinical Biochemistry and Pathobiochemistry, Charles University $2^{\text {nd }}$ Medical School and University Hospital Motol, Prague, Czech Republic

${ }^{2}$ Department of Chemistry and Biochemistry, Mendel University of Agriculture and Forestry, Brno, Czech Republic

${ }^{3}$ Clinic of Neurosurgery, Charles University $3^{\text {rd }}$ Medical School and University Hospital Kralovske Vinohrady, Prague, Czech Republic

${ }^{4}$ Department of Forensic Medicine, Charles University Prague, Czech Republic

Introduction: Primary traumatic brain injury (TBI) is followed by a sequence of destructive and neuroprotective cellullar and tissue metabolic changes. The aim of this study was to evaluate the correlation of metallothionein with time-profile of immune response in patients with TBI . Methods: 18 patients were divided into group I ( $\mathrm{n}=7$, expansive contusion), and group II ( $\mathrm{n}=11$, non-expansive contusion, and diffuse axonal injury). Serum interleukine-6, TNF- $\alpha$ and VEGF concentrations were measured by microchip assay, serum MT concentration by electrochemical technique. Cortical biopsies were investigated immunohistochemically and by electron microscopy. Results: Values of MT in the blood of group I were significantly lower compared to group II within 1 up 6 days after admission $(0.8424 \pm 0.2619$ vs. $1.445 \pm 0.1484 \mu \mathrm{mol} / \mathrm{l})$. Higher values of interleukine- 6 and lower values of VEGF were found during 1-6 days, and higher levels of TNF- $\alpha$ during 2-5 days in group I. We observed an increase of MT concentration during 4-6 days compared to values during 1-3 days in both, focal and diffuse TBI. Higher values of MT during hospitalization were found in patients with diffuse TBI during 4-6 days. Ultrastructural and immunohistochemical changes appeared within 24-48 hours including the loss of GFAP, Ki-67, and vimentin reactivity in expansive contussions. Conclusion: The loss of GFAP $(+)$ reactive astrocytes in expansive contusion might be associated with lower levels of MT. The work was supported by IGA CR, reg.no. NR/8793-3/2006. 


\section{FECAL OCCULT BLOOD TEST (FOBT) - COMMON GUAIAC VERSUS IMMUNOLOGICAL TEST}

Heinz Jürgen Roth, Heinrich Schmidt-Gayk

Limbach Laboratory, Im Breitspiel 15; 69126 Heidelberg, Germany

Colorectal Cancer (CRC) is one of the leading causes of cancer-related mortality in Germany. Although early detection is the primary option to reduce CRC mortality, compliance with current screening recommendations is poor. Large randomized clinical trials have consistently proven that annual screening with FOBT can reduce CRC mortality by up to $33 \%$. The commonly used guaiac-based FOBT detects the pseudoperoxidase activity in hemoglobin when it interacts with a guaiac-impregnated card in the presence of a hydrogen peroxide developer. The necessity of dietary restrictions before stool sampling remains unclear but false positive results can occur with plant peroxidase or dietary hemoglobin as well as false negatives with high doses of vitamin C. Being specific for human hemoglobin the immunological FOBT (iFOBT) does not require dietary restrictions which may potentially improve participation in screening programs. Contrary to guaiac-based FOBT, i-FOBTs do not detect blood from upper gastrointestinal (GI) bleeding because of degradation of hemoglobin or globin by upper GI enzymes, which significantly enhances the specificity for CRC using the i-FOBT. Simplification of stool sampling by means of special fecal sampling devices in combination with i-FOBT will improve acceptance by patients and compliance in participation in screening programs. Additionally quantification of fecal hemoglobin enables the clinicians to choose an i-FOBT threshold that best suits the clinical population examined. Since cancer and adenomatous polyps may bleed intermittently multiple stool samples from consecutive bowel movements may be needed in order to minimize the possibility of missing blood in the stool. In addition, periodic screening (annually, biannually) will enhance overall sensitivity as well as early detection and removal of large adenomatous polyps which impact the incidence of CRC. As hemoglobin is very unstable in neat stool, samples have to be further processed immediately after sampling and kept in hemoglobin-stabilizing (extraction-) buffer under well defined storage conditions until being processed with the i-FOBT.

I-FOBTs are non-reactive with non-human hemoglobin, drugs, vitamins, or peroxidase from food sources, or with blood from the upper GI, and they are more patient-friendly and better in specificity and sensitivity than guaiac-based FOBT (Sieg, Eur J Cancer Prev 1998;7:279-85). We will present the results with a new i-FOBT obtained in 725 patient samples including 186 patients with CRC, 171 patients with adenomatous polyps, 257 GI-healthy controls, and 74 disease controls.

\section{S17_A2}

\section{VIRTUAL VERSUS REAL COLONOSCOPY}

\section{Thomas Mang}

Department of Radiology; Medical University of Vienna; Waehringer Guertel 18-20; 1090 Vienna, Austria

Computed tomographic colonography (CTC) is a minimally invasive technique for imaging the entire colon. Based on a helical thin-section CT of the cleansed and air-distended colon, two-dimensional and three-dimensional projections are used for image interpretation. Several clinical improvements in patient preparation, technical advances in CT, and new developments in evaluation software have allowed CTC to develop into a powerful diagnostic tool.

CTC is already well established as a reliable diagnostic tool in symptomatic patients, after incomplete conventional colonoscopy (CC), in patients with obstructive cancer or when CC is contra-indicated or refused by the patient. Many experts currently consider CTC a comparable alternative to CC, although there is still debate about its sensitivity for the detection of colonic polyps in a screening population. As in large multi-centre trials controversial results were obtained, acceptance of this indication on a large scale is still pending. However, agreement exists that in experienced hands screening can be performed with CTC and new multi-centre trials are in progress. There are several current limitations that will need to be overcome to evolve the ultimate role of this technique in a screening program. There is currently considerable variability in the materials and methods used in CTC. Standardisation of reporting, implementation of cutoff sizes, and management of extracolonic findings are currently under debate. Ongoing research in computer assisted detection and new imaging software tools have the potential to increase accuracy and ease of interpretation.

This presentation summarizes the main indications for CTC as well as recent technical improvements and reviews the performance of CTC in clinical studies. 
MOLECULAR DNA DIAGNOSIS OF CRC

Bela Molnar

II. Dept. of Medicine, Semmelweis Uni, Budapest, Hungary

The early detection, diagnosis or follow-up of CRC lacks reliable tests.

Attempts to use fecal blood or peripheral blood protein markers could not yield a satisfactory diagnostic sensitivity and specificity. DNA is a stable, easily amplified and detectable molecule that can serve as reasonable target in faeces or blood.

Single target DNA testing in faeces is based today on K-ras or p53 genes. Sensitivity upto $58 \%$ could be achieved. Multipanel DNA markers (K-ras, APC, p53, Bat-26 and L-DNA) in stools were recently introduced with 64 to $91 \%$ sensitivity (95\% Conf. Interval, $71-99 \%$ ) and $93 \%$ specificitiy (C.I $88-100 \%)$. The already commercially available gene test is in routine use in the USA, while other DNA based fecal tests are in development for HNPCC.

Cell free DNA may range in the plasma or serum from 25 to $1000 \mathrm{ng} / \mathrm{ml}$ and can be used for CRC diagnostics. Serum was shown to contain a higher quantity and qualitiy of DNA as compared to plasma. Mutations of the K-ras, p53, APC, p16 INK, BRAF genes were evaluated using PCR, DHPLC, RFLP and recently by short oligonucleotide mass analysis(SOMA), bead emulsion amplification magnetics(BEAMing) and loop-mediated isothermal amplification. The highest sensitivity and specificity was achieved by the parallel analysis of these genes. In this case sensitivity of $46 \%$ to $67 \%$ with specificity upto $100 \%$ could be achieved.

Multi panel cDNA chip diagnostics of blood expression profiling became available in the last years. Early results show $60-90 \%$ sensitivity and $80-100 \%$ specificity.

A promising alternative is the oligo or cDNA chip based diagnostics for differential diagnosis, prognosis and therapeutic sensitivity in colon biopsy specimens.

Molecular DNA based fecal screening of CRC is already in the routine phase, with further technical developments multigene blood based screening and diagnostic tests are expected to appear.

\section{S17_A4}

\section{DNA METHYLATION IN CRC}

\section{Kolligs Frank T.}

Frank Kolligs, M.D., Department of Medicine II, University of Munich, Marchioninistrasse 15, 81377 Munich, Germany

Methylation of $\mathrm{CpG}$ islands of regulatory units of genes is thought to play a key role in tumor initiation. Many tumors reveal simultaneous and intense hypermethylation of multiple genes, a phenomenon that has been termed "CpG island methylator phenotype" (CIMP). CIMP was first described based on the finding of concordant methylation events in a subset of colorectal tumors. CIMP+ cases have been found to be more common in women, to occur more frequently in the proximal than in the distal colon as well as to present with a poorly differentiated histology and a distinct profile of genetic abnormalities. The presence of simultaneous methylation of multiple genes is associated with poor prognosis in many malignant tumors including colorectal cancer. The methylation status of certain genes has been analyzed in primary colorectal cancers as well as in serum and stool derived from patients with colorectal cancer and some studies have been published, reporting the feasible application of DNA methylation as a marker for screening and serum based prediction of prognosis in patients with colorectal cancer. Several studies have focussed on the detection of methylated DNA in stool. With assays based on the analysis of the methylation status of one or two genes detection rates for colorectal cancer of 42 to $96 \%$ and for adenoma of 31 to $81 \%$ have been reached. Fewer studies have evaluated the potential of methylation of serum DNA to monitor therapy or determine prognosis in patients with colorectal cancer. More and especially prognostic studies are warranted. In the future, analysis of methylated DNA might prove useful for early detection and prediction of prognosis of colorectal cancer. 
S17_A5

PROTEOMICS IN CRC

Georg J. Arnold, Friedrich Lottspeich,Thomas Fröhlich

Laboratory for Functional Genome Analysis,

Ludwig-Maximilians-University Munich, D-81377 Munich, GERMANY

The diagnosis of diseases based on the quantitative determination of individual proteins in body fluids is a well established and common method in clinical chemistry and usually based on assays specific for a given protein, e.g., enzymatic activity or antibody specificity. The development of new analytical methods for protein sequencing and identification by mass spectrometry, generally referred to as proteomics, substantially accelerated and simplified identification and quantification of almost any protein even in complex mixtures. As a consequence, systematic screening approaches on the protein level can now be applied for the search of new biomarkers. Proteomic screening, uses either a separation approach based on well known twodimensional gel electrophoresis in combination with covalent fluorescent labelling for quantification, or a chromatography based separation setup in which the quantification is performed by mass spectrometry using, e.g., stable isotope labelling. Nevertheless, both the extreme biochemical heterogeneity of proteins as well as their huge abundance range (at least 10 orders of magnitude in blood) limits the number of protein species which can practically be identified within typical screening projects. Several approaches for biomarker discovery in CRC on the basis of proteome techniques will be critically discussed with respect to their specific advantages and limitations. Besides application of proteomic techniques in basic research and biomarker discovery, mass spectrometry of proteins may soon be routinely used for high throughput quantification of distinct protein species. For this application, so-called MRM procedures are currently developed for a number of proteins.

S17_A6 PLASMA TIMP-1 COMBINED WITH PLASMA CEA MEASUREMENTS IN RISK ASSESSMENT FOR
CRC. A POPULATION BASED, PROSPECTIVE STUDY OF 5,005 INDIVIDUALS AT RISK FOR CRC.

\author{
Nielsen HJ*, Brünner N, Davis G, Stieber P, Blankenstein MA, Dowell B, Doss R, Christensen IJ
}

*Dept. of Surgical Gastroenterology, Hvidovre University Hospital, 2650 Hvidovre, Denmark.

Previous retrospective results suggest that TIMP-1 determined in plasma may be a biomarker for early detection of CRC and in particular of CC. It was also suggested that the combination of TIMP-1 and CEA could improve detection. We therefore initiated a prospective, population based study to test these hypotheses.

Individuals referred to endoscopy due to symptoms or other risk factors for CRC were included. Each individual donated a blood sample for serum, plasma and white blood cells, and baseline variables including life style variables, relevant previous and/or concurrent diseases and current medication were registered. The findings of the examination were registered using ICD-10 codes. TIMP-1 and CEA were determined in plasma from 5,005 individuals.

The findings were based on sigmoidoscopy in 1,787 and colonoscopy in 3,218 individuals. One hundred and eighty nine colon cancers (3.8\%) and 114 rectal cancers (2.3\%) were found. In addition to CRC, 10 patients with other cancers, 933 with adenomas and 1,461 with other findings (Colitis, Crohn's, diverticulosis, etc.) were found. Previous or concurrent diseases, such as other malignant diseases than CRC, diabetes, ischemic heart disease, arthritis, etc., were recorded in 2,593 individuals. A preliminary univariate analysis shows at $95 \%$ specificity:

\begin{tabular}{|c|c|c|c|}
\hline TIMP-1: & $\mathrm{OR}=2.8(2.3-3.3)$ & $\mathrm{AUC}=0.71$ & Sensitivity: $18 \%$ \\
\hline CEA: & $\mathrm{OR}=3.8(3.2-4.4)$ & $\mathrm{AUC}=0.73$ & Sensitivity: $33 \%$ \\
\hline \multicolumn{4}{|c|}{ CRC $(n=303)$ vs. no findings or current other diseases $(n=1,375)$ : } \\
\hline TIMP-1: & $\mathrm{OR}=7.9(5.9-10.5)$ & $\mathrm{AUC}=0.80$ & Sensitivity: $29 \%$ \\
\hline CEA: & $\mathrm{OR}=4.4(3.6-5.4)$ & $\mathrm{AUC}=0.77$ & Sensitivity: $36 \%$ \\
\hline \multicolumn{4}{|c|}{ CC detected by colonoscopy ( $\mathrm{n}=123$ ) vs. no findings or current diseases $(\mathrm{n}=836)$ : } \\
\hline TIMP-1: & $\mathrm{OR}=13.8(8.6-22.0)$ & $\mathrm{AUC}=0.85$ & Sensitivity: $46 \%$ \\
\hline CEA: & $\mathrm{OR}=4.3(3.1-5.8)$ & $\mathrm{AUC}=0.76$ & Sensitivity: $36 \%$ \\
\hline
\end{tabular}

A multivariate analysis of the set of subjects undergoing colonoscopy and presenting with no concurrent diseases and with $\mathrm{CC}(\mathrm{n}=54)$ as the endpoint compared to all others $(\mathrm{n}=1,422)$, that is other findings or no findings, demonstrated that TIMP1 and CEA adjusted for age and gender independently predict CC. The AUC is 0.82 and OR is 7.2 (95\% CI: 3.9-13.1) for TIMP-1 and 2.3 (95\% CI: 1.5-3.6) for CEA. The sensitivity at 95\% specificity was $37 \%$.

These results suggest that TIMP-1 in combination with CEA could be useful in the assessment of risk for colon cancer. 
S17_A7

MULTIPARAMETRIC ANALYSIS IN CRC

$\underline{\text { Petra Stieber }}^{1}$, Donna P Ankerst ${ }^{2}$, Fritz Spelsberg ${ }^{3}$, Ulla Lau-Werner ${ }^{3}$, Volker Heinemann $^{4}$, Dorothea Nagel ${ }^{1}$

${ }^{1}$ Institute of Clinical Chemistry, University of Munich, Klinikum Grosshadern, Germany; ${ }^{2}$ Institute for Medical Informatics, Biometry and Epidemiology, ${ }^{3}$ Surgical Department, ${ }^{4}$ Medical Department II; University of Munich, Klinikum Grosshadern, Germany

${ }^{1}$ Klinikum der Universität München - Großhadern, Marchioninistraße 15, 81377 München, Germany

Aim:To evaluate the gain in colorectal cancer diagnosis by combining multiple tumor markers.

Material and Methods: Levels of CEA, CA19-9, CA72-4, CYFRA21-1, BHCG, NSE, CA125, CA15-3, AFP(Elecsys Roche), and CA 242 (Canag Elisa) from 605 patients with colorectal cancer (127 UICC I, 175 UICC II, 144 UICC III, 159 UICC IV ) at time of diagnosis before first treatment and of 266 healthy individuals were assessed. Area underneath receiver operating characteristic curves (AUC) and sensitivities for fixed cut-offs obtaining $95 \%$ specificity were calculated for each marker. Tumor marker values were transformed by fractional polynomial-exponential functions and optimally combined by logistic regression (LR). Classification rule was externally evaluated by 5 -fold cross-validation and the average area underneath the receiver-operating characteristic curve (AUC) with standard deviation (SD), and sensitivity corresponding to the predicted probability cut-off obtaining $95 \%$ specificity were calculated.

Results: The strongest correlations for healthy individuals were between CA19-9 and CA242 (0.8) and between age and BHCG (0.55) as well as AFP (0.47). In cancers strong correlations were observed between CA 19-9 and CA 242 (0.88), CEA and CYFRA21-1 (0.52), CA72-4 (0.41), and CA242 (0.47), respectively, as well as between CA72-4 and CYFRA21-1 (0.47). The markers CA15-3 and AFP had no discriminating power for colorectal cancer, CA242 (0.63), NSE (0.62) performed slightly better, and CYFRA21-1 (0.79) and CEA (0.81) had the highest AUC. At 95\% specificity the highest sensitivities were found for CEA ( 47\% ) and CYFRA 21-1 (40\%).

In multivariate analysis LR obtained an AUC (SD) of $0.96(0.008)$ and a sensitivity of $72 \%$ at $95 \%$ specificity, statistically significant markers in the multiple LR included CEA, CYFRA21-1 and CA125.

Conclusion: Combination of the tumor markers CEA, CYFRA21-1 and CA125 leads to considerable improvement of diagnosis of colorectal cancer over any single marker. 


\title{
EUROPEAN RANDOMIZED STUDY (ERSPC): DOES SCREENING DETECT TOO MANY PROSTATE CANCERS?
}

\author{
Chris H. Bangma, Roderick van den Bergh, Fritz Schröder, Monique Roobol \\ Erasmus MC, Department of Urology, H1098, PO Box 2040, 3000 CA Rotterdam, The Netherlands
}

The incidence of prostate cancer (PC) has risen in most Western and Eastern countries during the last 15 years. Most detected tumors have a lower grade and stage than in the past. The increasing number of biopsies, the increasing number of cores per biopsy, the increasing overall life expectancy and most importantly the increasing use of PSA-measurements as a screening test, with lower thresholds for biopsy, are accountable for this development. The majority of these screendetected tumors have favourable characteristics, with a beneficial long-term survival [1]. $50 \%$ of these malignancies would most probably not have caused any symptoms during lifetime if they had remained undiagnosed. This so-called overdiagnosis due to screening often results in overtreatment, subjecting men to unnecessary costly and invasive treatment with the risk of important side effects. Men screened for prostate cancer should be protected against this. The replacement of initial active treatment with active surveillance in patients with small, localized, well-differentiated prostate cancer contributes to achieving this aim. Furthermore, in case of deferred treatment, quality of life might be increased during the initial observation period. Because screening for prostate cancer is frequently applied, the attention to this approach in this specific subgroup of men with prostate cancer has increased. There is a rising demand for an evidence-based approach, but unfortunately until this day it is not yet available. Uncertainties currently exist concerning the risk of missing the window of curability in prostate cancer and criteria to rely on for changing from active surveillance to curative therapy in time to avoid or minimize that risk [2].

In the Rotterdam section of the ERSPC (European Randomized study of Screening for Prostate Cancer [3]) and the Department of Urology of the Erasmus Medical Center in Rotterdam a retrospective study has performed on men detected with prostate cancer in the first round of screening. 261 out of 1,014 men $(25,7 \%)$ were suitable for active surveillance, based on internationally accepted histological and biochemical criteria for minimal cancers. In practice in only 64 of these 261 men $(24,5 \%)$ a watchful strategy was chosen [4]. In patients in whom active surveillance was actually elected, a very beneficial outcome was observed, with a cause-specific survival of $100 \%$. This illustrates that many cancers should not have been detected by screening. We have initiated a prospective, observational PRIAS-study (Prostate cancer Research International: Active Surveillance) to validate the management of prostate cancer with active surveillance [www.priasproject.org].

Acknowledgements. ERSPC is supported by the European Commission Sixth Framework Programme (contract no. LSHCCT-2004-503011). 


\title{
HOW DOES PSA TESTING IMPACT QUALITY OF LIFE?
}

\author{
Ida Korfage, Marie-Louise Essink-Bot
}

Erasmus MC University Medical Center Rotterdam, PO Box 2040, Rotterdam, 3000 CA, The Netherlands

Our presentation considers the quality of life effects of PSA-testing. We will present own research as described below and an update of recent literature on this subject.

Population-based screening for prostate cancer is currently being evaluated in randomized clinical trials in the United States and in Europe. Side effects arising from the process of screening and from the earlier treatment of screen-detected prostate cancer may be important factors in the evaluation. To examine health-related quality of life (or health status) in men during the screening process for prostate cancer, we conducted a longitudinal study of 626 attenders to the Rotterdam (The Netherlands) prostate cancer screening program and of 500 nonparticipants.

Attenders of the screening program and nonparticipants completed self-assessment questionnaires (SF-36 [i.e., Medical Outcomes Study 36-Item Short-Form Health Survey] and EQ-5D [i.e., EuroQol measure for health-related quality of life] health surveys) to measure generic health status, as well as an additional questionnaire for anxiety and items relating to prostate cancer screening. The attenders completed questionnaires at T1 (baseline) and T2 (waiting room) and either at T3 (when nothing suspicious was found in the initial screening tests) or at T4 (during the waiting period for the biopsy result) and T5 (after being informed that the biopsy result did not confirm a prostate cancer diagnosis).

Physical discomfort during digital rectal examination and during transrectal ultrasound was reported by $181(37 \%)$ of 491 men and by 139 (29\%) of 487 men, respectively; discomfort during prostate biopsy was reported by 64 (55\%) of 116 men. Mean scores for health status and anxiety indicated that the participants did not experience relevant changes in physical, psychological, and social functioning during the screening procedure. However, high levels of anxiety were observed throughout the screening process among men with a high predisposition to anxiety. Similar scores for anxiety predisposition were observed among attenders and nonparticipants. At the group level, we did not find evidence that prostate cancer screening induced important short-term health-status effects, despite the short-lasting side effects related to the biopsy procedure. However, subgroups may experience high levels of anxiety. The implication is that unfavorable health-status effects of prostate cancer screening occur mainly in the treatment phase.

\section{S18_A3}

\section{PCA3: A MOLECULAR URINE PROSTATE CANCER TEST}

\author{
Harry Rittenhouse \\ Gen-Probe Incorporated, 10210 Genetic Center Drive, San Diego, CA 92121, USA
}

PCA3 is a prostate tissue-specific gene that is highly over-expressed in over $85 \%$ of prostate tumors with a median 66-fold up-regulation compared with non-cancer prostatic tissue. Using molecular diagnostic technology, Gen-Probe has developed a quantitative assay in urine for PCA3 mRNA expression. Following a DRE, voided urine is measured for quantitative PCA3 mRNA and PSA mRNA. The ratio of PCA3 mRNA to PSA mRNA represents the PCA3 score. Clinical research studies have been in progress to assess specificity/sensitivity for biopsy outcome, effect of prostate volume and tumor volume, biological variation and reproducibility in different laboratories. Multiple studies have shown that the PCA3 test is significantly more accurate than serum PSA especially for patients with previous negative biopsies. Unlike serum PSA, the PCA3 test is not correlated with prostate size but is correlated with tumor volume. The biological variability is relatively low and consistent results are obtained for patients in serial analysis. The PCA3 assay has been shown to be highly reproducible in several different laboratories with very similar ROC analysis. Since the PCA3 test has a completely independent mechanism for cancer detection compared to serum PSA, the two tests together with other prostate parameters are expected to act synergistically. Recent preliminary studies have revealed that PCA3 is a very significant addition to algorithm analysis with Free and Total PSA. 


\title{
DNA METHYLATION MARKERS FOR DIAGNOSIS AND PROGNOSIS OF PROSTATE CANCER
}

\author{
Ralf Lesche, Shannon Payne, Fabian Model, Gunter Weiss, and Andrew Sledziewski \\ Epigenomics AG, Kleine Präsidentenstr. 1, 10178 Berlin, Germany \\ Epigenomics Inc, 1000 Seneca St, Ste 300, Seattle, WA 98101, USA
}

\begin{abstract}
Aberrant DNA methylation is an early, common, and stable event during tumorigenesis, that can be assayed in cancer tissue and body fluids, making it an attractive target for biomarker development. We have identified DNA methylation biomarkers for early disease detection and molecular classification of prostate cancer.

A biomarker with improved specificity relative to serum PSA would offer a valuable tool for prostate cancer diagnosis. We have identified DNA methylation biomarkers with the power to discriminate prostatic adenocarcinoma from benign epithelium. Highly sensitive, quantitative real-time PCR assays were developed using the HeavyMethyl (HM) technology. A clinical study was initiated to determine the performance characteristics of markers associated with the genes GSTP1, RASSF2, HIST1H4K, and TFAP2E in body fluid analytes. Plasma and urine were collected from 91 prostate cancer patients, 51 patients with negative biopsy, and 50 young healthy men. Receiver operator characteristic (ROC) curves were generated for each marker and the DNA methylation level of the candidate markers were correlated with established clinical parameters. Our study suggests that higher sensitivity for early stage detection of prostate cancer can be reached with urine specimens.

Despite radical prostatectomy as a first line treatment $15-20 \%$ of prostate cancer patients experience biochemical recurrence manifested by rising PSA levels within five years of surgery. Current prognostic indicators, mainly based on histopathological factors cannot sufficiently define the risk for biochemical recurrence. We have measured the DNA methylation levels of six marker candidates (ABHD9, CCND2, Chr3-EST, GPR7, HIST2H2BF, PITX2) using formalin fixed paraffin embedded tissue (FFPE) sections from a cohort of 605 patients who underwent radical prostatectomy. Assuming a Cox proportional hazard model we found several markers to be significant predictors of biochemical recurrence. DNA methylation of PITX2 was the strongest predictor. Patients with above average PITX2 methylation were four times more likely to experience biochemical recurrence within eight years after surgery when compared to patients with below average methylation. Notably, the PITX2 biomarker predicts outcome in the intermediate risk group (Gleason 7) of patients, for which reliable prognosis is currently challenging.
\end{abstract}

\section{S18_A5}

\section{P-MARK: A EUROPEAN QUEST FOR NEW DIAGNOSTIC AND PROGNOSTIC MARKERS FOR PROSTATE CANCER}

\author{
Ellen Schenk-Braat and Chris H. Bangma
}

Erasmus MC, Department of Urology, JNI room Be362, PO Box 2040, 3000 CA Rotterdam, The Netherlands

\begin{abstract}
Aims:
Prostate cancer is the most frequent malignancy in European men, and its incidence and mortality is increasing. There is a growing need for improved diagnostic and prognostic biomarkers to limit overdiagnosis and overtreatment due to opportunistic screening and to identify men at risk for life-threatening disease. P-Mark is a European project funded by the EU Sixth Framework Programme and is aimed at the identification of novel biomarkers as well as the evaluation and validation of recently developed promising biomarkers for prostate cancer [1]. The P-Mark consortium is composed of seven academic and two industrial partners and is coordinated by Prof. dr. Chris Bangma (Erasmus MC, The Netherlands). The project has started on 1 November 2004 and is now entering its final phase.

\section{Methods and Results:}

A P-Mark biorepository has been established that is composed of over 1900 historical serum samples and 400 historical urine samples. Currently, the biorepository is being extended with matching blood and urine samples that are prospectively collected at four clinical centres. Using sample sets from the P-Mark biorepository, candidate biomarkers have been identified in serum with innovative mass spectrometry tools. Immunoassays are being developed that will be used to validate these novel biomarkers. Based on the outcome of evaluation studies on recently developed promising biomarkers, human kallikrein type 2 (hK2), nicked PSA, osteoprotegerin (OPG) and PCA3 have been selected for further validation. Across marker studies in large patient cohorts are ongoing. In addition, these markers will be validated in the screening setting of the European Randomized Study of Screening for Prostate Cancer.
\end{abstract}




\section{Conclusions:}

The results of the validation studies carried out in P-Mark will indicate if hK2, nicked PSA, OPG and PCA3 can add to risk stratification for prostate cancer. Furthermore, the P-Mark biorepository is unique in its kind and will be of exceptional value for the urological scientific society.

Acknowledgements.

P-Mark is funded by the European Commission Sixth Framework Programme (contract no. LSHC-CT-2004-503011).

\section{S18_A6}

\section{EFFECTS OF PSA ASSAY STANDARDIZATION ON CLINICAL DECISION MAKING}

\section{Axel Semjonow}

Prostate Center, University Clinic Münster, Albert-Schweitzer-Str. 33, D-48149 Münster, Germany

Comparative data between five total PSA methods (Abbott AxSYM, Bayer Centaur, Beckman Coulter Access, DPC Immulite and Roche Elecsys) have recently been published using a serum population of 596 samples (Stephan et al, Clin Chem 2006; 52:1,59-64). One result of the comparison illustrates the bias between artificial and clinical samples: Roche Elecsys reports about 20\% lower results on artificial samples as compared to Beckman Coulter Access in the study published by Roddam et al, Ann Clin Chem 2006;43:35-48. The results on clinical samples show that Roche Elecsys median total PSA value as calculated from 596 samples is 7\% higher as compared to Beckman Coulter Access. Thus, the observed bias between artificial and clinical samples is about $25 \%$ across the two methods.

Roddam et al. suggest that clinical errors arise from PSA miscalibration. However, WHO calibration alone has no impact on improvement of clinical interpretation of the results, but appears to result in about $15 \%$ lower PSA recovery in some of the assays. Both studies (Roddam and Stephan) show that parity is not achieved even after WHO calibration. Guidelines from the European Group on Tumor Markers and from the National Academy of Clinical Biochemistry, state that clinically validated and assay-specific PSA cut-off values should be used to allow physicians to draw correct conclusions. Indeed, clinical errors will occur when non assay-specific and non clinically-validated cut-off values are selected. 
DISSECTING CELL FATE REGULATION IN STEM CELLS

Lemischka Ihor

Dept. of Molecular Biology, Princeton University, Princeton NJ 08544, USA

The mechanisms that control cell-fate decisions in stem cells are not well understood. We must unravel the fundamental regulation of self-renewal versus differentiation in order to realize the tremendous biomedical potential of these cells. Our laboratory pursues this issue in both somatic and embryonic stem (ES) mouse cells. We have completed studies that define the molecular "signature" of hematopoietic stem cell (HSC) and ES cell populations. These signatures represent broad panels of gene-products whose expression is correlated with primitive stem cell states. We utilized comprehensive inhibitory RNA techniques facilitated by lentiviral vector mediated transduction of short hairpin RNA expressing cassettes into HSC and ES cell populations. Primarily, we have down-regulated the expression of numerous transcriptional regulators in these cells. Our most advanced experiments have identified gene-products that are required for the maintenance of self-renewal and pluripotency in ES cells. Coupled with extensive analyses of the modified ES cells in terms of distinct differentiation pathways, as well as genome-wide microarray experiments, we propose a novel model of ES cell regulation. Our systems allow the perturbation of individual gene-products in a controlled manner. We have applied these techniques to analyze the dynamics of cell-fate decisions at the chromatin, transcriptional, and proteomic levels.

\title{
S19_A2
}

\section{GENETIC SIGNATURES OF "STEMNESS" PATHWAYS IN THERAPY-RESISTANT HUMAN CANCERS AND THEIR THERAPEUTIC IMPLICATIONS}

\author{
Gennadi V. Glinsky
}

\begin{abstract}
Translational \& Functional Genomics Laboratory, Ordway Cancer Center, Ordway Research Institute, Center for Medical Science, 150 New Scotland Avenue, Albany, NY 12208; Department of Surgery, Division of Urology, and Department of Pathology \& Laboratory Medicine, Albany Medical College, Albany, NY 12208; Sidney Kimmel Cancer Center, San Diego, CA 92121
\end{abstract}

In clinical terms, all human cancers diagnosed in individuals can be divided in two major categories: malignant tumors that will be cured with the existing cancer therapies and tumors that have therapy-resistant phenotypes and will return after initial treatment as incurable metastatic disease. These tumors manifesting clinically lethal death-from-cancer phenotypes represent the most formidable challenge of experimental, translational, and clinical cancer research.

Clinical genomics data demonstrate that gene expression signatures associated with the "stemness" state of a cell are informative as molecular predictors of cancer therapy outcome and can help to identify cancer patients with therapy resistant tumors (1-4). Here we present experimental and clinical evidence in support of the BMI1 pathway rule indicating a genetic link between the "stemness" state and therapy-resistant death-from-cancer phenotypes. Our analysis demonstrates that therapy-resistant and therapy-responsive cancer phenotypes manifest distinct patterns of association with "stemness"/ differentiation pathways, suggesting that therapy-resistant and therapy-responsive tumors develop within genetically distinct "stemness"/differentiation programs. These differences can be exploited for development of prognostic and therapy selection genetic tests utilizing microarray-based cancer therapy outcome predictor (CTOP) algorithm.

Discovery of death-from-cancer signature genes implies that genetic signatures associated with a "stemness" state (defined as phenotypes of asymmetrical division, pluripotency, and self-renewal) might be informative as molecular predictors of cancer therapy outcome. We tested the validity of this concept employing genome-wide microarray analysis of several models of differentiation of human and mouse ESC. Applying signature discovery principles to analysis of gene expression during transition of ESC from "stemness" to differentiated phenotypes, we identified nine gene expression signatures associated with a "stemness" epigenetic program of ESC that appear highly informative in stratification of the early-stage breast, prostate, and lung cancer patients into sub-groups with statistically distinct probabilities of therapy failure. Cancer therapy outcome predictor (CTOP) algorithm employing a panel of "stemness' signatures [signatures of Nanog/Sox2/Oct4-, EED-, BMI1-, and Suz12-patways; transposon exclusion zones (TEZ) and bivalent chromatin domains (BCD) signatures] and demonstrates nearly $100 \%$ specificity and sensitivity of CTOP power in retrospective analysis of large independent cohorts of breast, prostate, lung, and ovarian cancer patients. To date, the retrospective analysis of the prognostic power of individual "stemness" signatures is being extended to more than 3,100 patients diagnosed with 12 
distinct types of cancer. One of the major regulatory pathways manifesting distinct patterns of association with therapyresistant and therapy-responsive phenotypes is the Polycomb group ( $\mathrm{PcG}$ ) proteins chromatin silencing pathway (5). RNAi-mediated targeting of the critical regulatory components of the PcG pathway in metastatic cancer cells eradicates disease in $67-83 \%$ of animals in a fluorescent orthotopic model of human prostate cancer metastasis in nude mice (5). We conclude that genetic signatures of "stemness" state identify therapy-resistant phenotypes in cancer patients diagnosed with multiple types of epithelial malignancies. These results provide powerful clinical evidence supporting the validity of the concept of cancer stem cells for human solid tumors.

\title{
S19_A3
}

\section{BREAST CANCER STEM CELLS: BIOLOGICAL AND CLINICAL IMPLICATIONS}

\author{
Gabriela Dontu, Christophe Ginestier, Emma Jauffret, Max Wicha
}

Comprehensive Cancer Center, Department of Internal Medicine, University of Michigan, Ann Arbor, Michigan 48109, USA,

Application of stem cell biology to cancer research has been limited by the lack of simple assays for identification and isolation of normal and malignant stem cells. We show that stem/progenitor cells in normal breast epithelium and breast tumors have increased aldehyde dehydrogenase 1 (ALDH) activity. Furthermore, immunostaining using ALDH1 antibody identifies normal and malignant stem/ progenitor cells in situ. In a series of 577 breast carcinomas on tissue microarrays, expression of ALDH1 was an independent predictor of poor prognosis. The cancer stem cell model has fundamental biological and clinical implications. We provide direct support for this model, by demonstrating that ALDH can be utilized as a marker to isolate and study stem cells from both normal and malignant human breast epithelium. These findings can also be used to study the progression of cells from normal to pre-malignant and then malignant state. Furthermore, we show that cancer stem cell concepts have clinical relevance. Expression of ALDH1 identifies a subset of breast cancer patients with poor clinical outcome. The techniques developed to detect breast cancer stem cells may be generally applicable in other malignancies, and may open the cancer stem cell field to clinical applications. 
THYMIDINE KINASE 1 AS MARKERS FOR CELL PROLIFERATION IN MALIGNANCIES; DESIGNED PEPTIDE ANTIBODIES USED FOR HISTOCHEMISTRY AND SERUM TK STUDIES

\author{
$\underline{\text { S. Eriksson }}{ }^{1,2}$, N. Wang ${ }^{2,3}$, Q. $\mathrm{He}^{4}, \mathrm{~S} . \mathrm{Skog}^{4}$ and B. Tribukait ${ }^{3}$ \\ ${ }^{1}$ Department of Anatomy, Physiology and Biochemistry, The Swedish University of Agricultural Sciences, BMC, PO \\ Box 575, SE 75123 Uppsala, Sweden 2.AroCell AB, Dag Hammarskjoldsv 34, SE-75237, Uppsala, Sweden ${ }^{3}$ Department \\ of Oncology and Pathlology, Karolinska University Hospital, SE 17177 Stockholm, Sweden ${ }^{4}$ Department of Oncology, \\ Karolinska University Hospital, SE 14186 Huddinge, Sweden
}

Cytosolic Thymidine kinase 1 (TK1) is a key enzyme in nuclear DNA precursor synthesis and antibodies against TK1 should be valuable tools for studies of cell proliferation. A high level of TK1 is a hallmark for cells in S-phase and increases in TK1 activity in sera from patients with malignancies has been used clinically as a marker in monitoring blood malignancies. However, no established methods using TK1 antibodies to follow cell proliferation has so far been available. Polyclonal chicken TK1 antibodies were produced with two peptides antigens based on the 3-D structure of TK, representing the active site and a C-terminal exposed region of human TK1. These antibodies were to study the TK1 expression in benign and malignant tissues and to compare the results with other markers of cell proliferation using immuno-histochemistry. A preliminary characterization of serum TK1 including a gel filtration size analysis of samples from breast cancer and lung cancer patients as well as cytosolic TK1 from cell culture cells have been done. The results show that TK1 expression can be evaluated in tonsils, and benign, intraepithelial neoplasia and carcinoma of the prostate with these TK1 antibodies. The size distribution of serum TK1 showed a broad peak corresponding to $700 \mathrm{kDa}$ and one more defined at 60-80 kDa. The two antibodies differed in reactivity with these peaks. Samples from different patients also showed a variation in the pattern of serum TK reactivity. Further, studies will reveal the usefulness of the TK1 antibodies in clinical oncology.

S20_A2

\title{
ULTRA SENSITIVE COLOROMETRIC THYMIDINE KINASE ACTIVITY ANALYSES USING TEMPLATE DEPENDENT PRODUCT IMMOBILISATION BY REVERSE TRANSCRIPTASE COMPLEMENTED BY YEAST KINASES
}

$\underline{\text { Jan-Simon Gronowitz }}$

Biovica AB, Uppsala Science Park, Dag Hammarskjölds väg 32 B, 75183 Uppsala, Sweden

The construction, function and capacity of the novel standardised TK-activity assay the so called DiviTum will be illustrated both considering enzymatic and clinical aspect.

The separation between the TK substrate Bromodeoxyuridine and the product BrdUMP, is obtained by further phophorylation of the product to BrdUTP by yeast kinases and by concomitant DNA synthesis by reverse reverse transcriptase using a template immobilised in the micro titre plate. After the all in one reaction engaging TK and another three enzymes the plate is washed and alkaline-phosphatase-anti-BrU antibody binding the new DNA is added followed by a second wash and colorimetric determination of alkaline phosphatase activity. Said activity is proportional to the TK activity in the original sample. The kinetics illustrating the measuring range for the technique shows that BrdUMP amount $<10^{-16}$ moles are detected, also corresponding to $<500 \mathrm{TK}$ molecules which in turn is the TK content of one dividing cell. The assay is capable of measuring the whole serum TK activity range from $2 \mathrm{ng} / \mathrm{L}$ up to $120000 \mathrm{ng} / \mathrm{L}$. Different results of applying the novel assay for following pathological cell division in cultures, animals and man will be presented. The independence of TK as a proliferation marker in contrast to classical tumor markers will be demonstrated by data showing that serum TK discriminate between "good and bad" PSA. It is concluded, albeit additional studies are required, that the novel assay will fill the gap in clinical chemistry for a serum based test measuring total pathological cell division in the body and thus detecting tumors at an early stage. 


\section{DEVELOPMENT OF AN AUTOMATED, NON-RADIOMETRIC ASSAY FOR THYMIDINE KINASE ON THE LIAISON ${ }^{\circledR}$ ANALYZER - TECHNICAL AND CLINICAL EVALUATION}

Jennifer Fenske, Alyssa Tuttle, Greg Olson, Anne-Charlotte Aronsson and Jane Schmidt

DiaSorin Inc., 1951 Northwestern Ave P.O Box 285, Stillwater, MN 55082, USA

Aims:Determination of Thymidine Kinase (TK) activity has been shown to be useful for prognosis and monitoring of patients with hematological malignancies. TK is involved in the synthesis of DNA and catalyses the phosphorylation of thymidine to thymidine monophosphate. The activity of TK increases during the S-phase of the cell cycle.

Methods:The LIAISON ${ }^{\circledR}$ Thymidine Kinase Assay is an automated non-radioactive assay for determination of TK that has been developed and is available from DiaSorin Inc. Serum TK has been measured by use of a commercially available radioenzyme assay (DiaSorin Prolifigen ${ }^{\circledR}$ TK REA).

Results and Conclusions:The assay range is 0.5 to $100 \mathrm{U} / \mathrm{L}$. Functional sensitivity is $\leq 5 \mathrm{U} / \mathrm{L}$. Precision results are $\leq 12 \%$ above $7 \mathrm{U} / \mathrm{L}$. Average recovery is $101 \%$. Dilution linearity gives a regression equation of observed $=0.94$ (expected) $+0.27 ; \mathrm{r}=0.997$.

Clinical samples from patients with different forms of hematological malignancies were measured in the LIAISON ${ }^{\circledR}$ Thymidine Kinase assay in an intersite study, and the results were compared to the TK REA and other clinical information. Clinical sensitivity was comparable to the TK REA and the regression equation was LIA $=0.769$ (REA) $+0.997 ; \mathrm{R}=$ 0.97.

From these data we conclude that LIAISON is a convenient, accurate and precise method for the quantitation of TK in human samples. It correlates to the REA and therefore provides a valuable tool in the management of hematological malignancies.

Product availability is subject to required regulatory approvals.

S20_A4

\section{THYMIDINE KINASE ENZYMATIC ACTIVITY - GOOD MARKER FOR THE EARLY RECOGNITION OF THE RELAPSE OF ACUTE LEUKEMIA IN CHILDREN}

Tomas Votava ${ }^{1}$, Ondrej Topolcan ${ }^{2}$, Lubos Holubec Jr. ${ }^{3}$, Zdenka Cerna ${ }^{1}$, Lumir Sasek ${ }^{1}$ and Stanislav Kormunda $^{2}$

${ }^{1}$ Department of Pediatrics Alej Svobody 80, Pilsen 300 00, Czech Republic

${ }^{2}$ Central Laboratory of Radioisotopes, ${ }^{3}$ Second Clinic of Internal Medicine and ${ }^{3}$ Department of Oncology and

Radiotherapy, Charles University, Faculty of Medicine and Faculty Hospital, Pilsen, Czech Republic

Aims:

Our main goal was to determine the significance of elevated serum TK1 enzymatic activity (TK1) as a relapse marker during follow-up with child patients suffering from acute leukemia.

Method:

TK1 serum levels in 44 children with acute leukemia (40 lymphoblastic, 4 myeloblastic) were determined using radioreceptor analysis (Immunotech, Prague, USA). All patients included in this study were tested for TK1 before the start of treatment and at least twice during the follow-up.

Results:

Our results showed that TK1 serum levels at the time of diagnosis were extremely high ( $78-5826 \mathrm{U} / 1$, median value 403 $\mathrm{U} / 1)$, while in remission TK1 serum levels were much lower $(5-80 \mathrm{U} / 1$, median value $31 \mathrm{U} / 1)$. During relapse of acute leukemia ( 6 cases) TK1 levels increased considerably to measurements between 120 - $800 \mathrm{U} / 1$ (median value $324 \mathrm{U} / 1$ ). The study showed that the elevation of TK1 serum levels during follow-up was a helpful marker for the recognition of an early stage of relapse and in some cases occurred as early as one month before the appearance of clinical signs. Sensitivity in this case was $87 \%$ and thus TK1 serum levels seem to be a very good parameter during follow-up because of acceptable sensitivity. Specificity we will discuss in the lecture in detail.

Conclusion:

TK1 serum enzymatic activity is a helpful marker in predicting relapse during follow-up in our group of patients with acute leukemia. 


\title{
THE CLINICAL VALUE OF THYMIDINE KINASE IN HEMATOLOGICAL MALIGNANCES
}

\author{
Nowrousian M. R. University Essen Innere Klinik Tumorforschung, Essen, Germany \\ Wiefelspütz J. University Essen Institut für Klinische Chemie \\ Anne-Charlotte Aronsson DiaSorin AB, Sweden \\ Elke Rauhut DiaSorin Germany
}

Thymidine kinase (TK) is a cell cycle-dependent marker that can be detected in the serum of patients diagnosed with many different types of cancer. TK is formed prior to cell division and it increases dramatically during the S-phase of the cell cycle. The activity of TK has been shown to be a reliable marker of the proliferative activity of tumor cells.

A large number of clinical studies has reported elevated TK levels in a variety of neoplasias, depending on tumor type and disease stage. The role of TK has been best studied in hematologic malignancies, particularly in low-grade non Hodgkin's lymphoma (NHL), high grade lymphoma, CLL and Hodgkin's disease.

Serum levels of thymidine kinase have been shown to reflect the progression of cancer as well as indicating of the efficacy of chemotherapeutic intervention. Determination of S-TK may therefore help to monitor the clinical course of the disease during therapy and predict the prognosis. Pretreatment serum TK levels have been shown to correlate with the clinical stage and allow discrimination between stable and progressive disease, even at early stage disease.

Most studies on TK have been made with a radioactive immunoassay (DiaSorin TK REA), which has limited a more widespread use, due to the cumbersome handling.

A new fully automated chemiluminescence assay for an easy and rapid measurement of TK has now been developed. A comparison was made between the two assays using 289 serum samples from 171 patients with NHL, 95 patients with Hodgkin's disease, and 23 patients with multiple myeloma. The results demonstrate a highly significant correlation with the TK REA indicating the reliability of the new test for use in clinical practice.

S20_A6

\section{THE DIAGNOSTIC AND PROGNOSTIC SIGNIFICANCE OF THYMIDINE KINASE (TK) IN TUMOR DISEASES}

O. Topolcan, S Svobodova, L.Holubec ${ }_{\mathrm{jr}}$, V Treska., S Kormunda, A Sutnar, K Rupert, M. Prazákova, M. Casova V. Polivkova, M. Spisakova,J. Finek.

Faculty Hospital in Pilsen, Dept. of immunoanalysis, E. Benese 13, 30599 Pilsen, Czech Republic

Introduction: The aim of our study was to evaluate the diagnostic and prognostic significance of Thymidine Kinase in tumors.

Material and Methods: Thymidine Kinase was measured using REA method (Immunotech,Prague). TK serum levels were measured in 1087 patients with solid tumors and in a control group of 500 persons. We have measured TK at the time of diagnosis, prior to surgery and immediately after surgery. Using TK measurement we have monitored the therapy.

Results: TK levels correlated with tumor aggressiveness at primary diagnosis, but did not correlate with tumor localization. Levels significantly increased immediately after the surgery with respect to the surgery effect and to the etiology. (benign or malignant). The increase correlated only with surgery extent and results from cellular proliferation. It has no diagnostic importance. TK is the only marker useful for monitoring therapy in colorectal cancer. TK serum levels were significantly increased in inflammatory and immuno-alteration diseases, compared to healthy probands.

Conclusion: Thymidine Kinase is a secondary tumor marker, useful particularly for monitoring the follow-up of tumor diseases. Elevated levels of this marker must be always interpreted together with a detailed knowledge of the patient's condition, because non-specific elevations of serum levels (inflammatory and immuno-alteration diseases) must be excluded.

This study was supported by research project 0021620819 and NR 8301-3/2005. 


\section{CLINICAL UTILITY OF THYIMIDINE KINASE (TK) IN LYMPHOPROLIFERATIVE MALIGNANCIES}

Vivian Barak, Moran Friedman, Moshe Gat, Inna Kalickman, Dinna Ben -Yehuda, Anne-Charllote Aronsson

Immunology Lab for Tumor Diagnosis, Oncology Dep., \& Hematology Dep., Hadassah Hebrew University Medical Center, Jerusalem, Israel

STUDY AIMS: To evaluate the sensitivity of the new TK marker as to treatment response monitoring, prediction of recurrence and prognostic significance.

METHODS AND PATIENTS: Patients with different lymphoproliferative malignancies were followed up for 4 years74 Multiple Myeloma (MM), 77 Non Hodgkin Lymphoma (NHL), 12 Hodgkin Disease (HD), 9 Chronic Lymphocytic Leukemia (CLL), 3 Chronic Myeloid Leukemia (CML), 5 Hairy Cell Leukemia (HCL) ,4 Acute Myeloid Leukemia (AML), 2 Acute Lymphocytic Leukemia (ALL) as well as 30 normal controls were studied for TK, compared to b2M and sIL-2R levels and correlated to disease status. .

RESULTS: Normal levels $(\mathrm{u} / \mathrm{L})$ of TK in 33 healthy individuals were found in a Range of Minimum- 1.8 and Maximum -8.7 with a Mean of $4.63+\mathrm{SE}-0.3$.

Patients with active disease (AD) from different groups of patients had significantly higher levels of TK (Range $3-87 \mathrm{u} / \mathrm{L}$ ), similar to $\mathrm{b} 2 \mathrm{M}$, as opposed to patients with no evidence of disease (NED), which had low, and similar levels as the controls. Increasing levels of TK indicated recurrence and were prognostic for survival. Therapy monitoring showed decreasing TK levels to correspond with therapy response.

CONCLUSIONS: We demonstrated TK as a very sensitive proliferation marker, indicating response to therapy,predicting survival and detecting earlier recurrence.

Further studies will elucidate the potential of TK based staging systems and providing prognostic information as compared to other available markers in lymphoproliferative malignancies. 


\title{
CLINICAL USE OF CIRCULATING TUMOR CELLS IN THE MANAGEMENT OF PATIENTS WITH BREAST CANCER
}

Herbert Fritsche

Lab Med Box 37, MD Anderson Cancer Center, Houston TX 77030, USA

\begin{abstract}
Aim:
The CellSearch ${ }^{\mathrm{TM}}$ system (Veridex, LLC) is designed to enrich and enumerate circulating tumor cells (CTCs) in peripheral blood. CTCs are identified as nucleated cells which express EpCAM and epithelial cytokeratins, but not CD45. This report describes the analytical performance of this system and our experience with the assay for clinical use in patients with metastatic breast cancer.
\end{abstract}

\section{Method:}

Analytical performance was assessed by intra and interassay precision studies using cell controls, recovery was assessed by spiking with 4-12 cells, and CTC stability was assessed by varying storage times and temperature. Clinical correlation studies were performed with samples from metastatic breast cancer patients.

Results: Intra- and inter-assay precision data showed $>95 \%$ of control values to be within the specified ranges. Recovery rate averaged between $80 \%$ and $100 \%$. When comparing CTC values of identical samples stored for 24 , 48, or 72 hours at room temperature or at $4{ }^{\circ} \mathrm{C}$, no statistically significant differences were observed, but greater variability of results was observed in the refrigerated samples. CTCs were detected in approximately $60 \%$ of metastatic breast cancer patients. Clinical correlation studies confirmed the prognostic and predictive utility of the CTC assay. Metastatic breast cancer patients who have more than 5 CTCs detected before therapy is initiated or after the first course of chemotherapy are in a poor prognostic category.

Conclusions: The CellSearch ${ }^{\mathrm{TM}}$ system enables the reliable quantitation of CTCs in blood and in concert with previous clinical reports, appears to be a suitable tool for the routine assessment of metastatic breast cancer patients.

S21_A2

\section{CLINICAL RELEVANCE OF THE MOLECULAR ANALYSIS OF CIRCULATING TUMOR CELLS (CTC)}

Winfried H. Albert, Michael Lustig, Silke Lankiewicz and Siegfried Hauch

AdnaGen AG, Ostpassage 7, D-30853 Langenhagen, Germany

Aims:

Circulating tumor cells (CTC) were investigated with respect to prognosis and therapy in patients with breast, colon or prostate cancers. Furthermore, the expression of specified markers in CTC was analysed in various clinical situations.

Method:

CTC are enriched from patient EDTA-blood with an antibody mix coupled to magnetic beads. This is followed by a multiplex RT-PCR, which enables the detection of tumor associated transcripts. This test design allows the detection of CTC with a high analytical sensitivity (one tumor cell in $5 \mathrm{ml}$ blood) by maintaining an excellent specificity ( $>90 \%)$ and is taking care of the extensive antigenic and transcriptional heterogeneity of the tumor cells.

Results:

The prognostic value of the determination of CTC has been widely published and accepted. With the above test system it was possible to monitor therapeutic efficacy or the development of drug resistance in M1 patients in real time, thus offering the physician the possibility to adjust the therapy accordingly. Also a correlation between expression profiles of CTC and tumor stages was recognized. Discrepancies in the expression of therapeutic targets in the primary tumor and CTC have been frequently noted, which might be important for choosing the right agent, e.g. antibody.

Conclusion:

The detection and the molecular analysis of CTC is a powerful diagnostic tool to describe the clinical situation of tumor patients and to possibly help to guide their therapy for a better disease outcome with respect to progression free and overall survival. 
ARTIFICIAL NEURAL NETWORKS AND NOMOGRAMS IN PROSTATE CANCER

\author{
F. K.-H. Chun ${ }^{1,2}$, P.I. Karakiewicz ${ }^{2}$, H. Huland ${ }^{1}$ \\ ${ }^{1}$ Department of Urology, University of Hamburg, Germany \\ ${ }^{2}$ Cancer Prognostics and Health Outcomes Unit, University of Montreal, Montreal, Quebec, Canada;
}

Aims:

Several nomograms have been developed to predict PCa related outcomes. Neural networks represent an alternative. Method:

We provide a descriptive and an analytic comparison of nomograms and neural networks, with focus on PCa detection. Results:

Our results indicate that nomograms have several advantages that distinguish them from neural networks. These are both quantitative and qualitative.

Conclusions:

In the field of PCa detection, nomograms appear to outweigh the benefits of neural networks. However, the neural network methodology represents a valid alternative, which should not be underestimated.

S22_A2

\title{
THE PROSTATE CANCER PREVENTION TRIAL PROSTATE CANCER RISK CALCULATOR
}

Donna Pauler Ankerst, Ian M. Thompson

Institute for Medical Informatics, Biometry and Epidemiology, University of Munich, 81377, Germany

Objective: To develop, validate and make publicly available on the internet a prostate cancer risk calculator based on prostate-specific antigen (PSA) and other established risk factors.

Methods: 5519 men from the placebo group of the Prostate Cancer Prevention Trial (PCPT) who underwent prostate biopsy and had at least one PSA measurement and a digital rectal exam (DRE) performed during the year before biopsy were used in a multivariable logistic regression correlating risk of prostate cancer and high-grade disease (Gleason grade $\geq 7$ ) with age, race, family history of prostate cancer, PSA level, PSA velocity, DRE and history of a prior negative biopsy.

Results: Higher logarithmic PSA level (odds ratio $[\mathrm{OR}]=2.34,95 \%$ confidence interval $[\mathrm{CI}]=2.13$ to $2.56, \mathrm{P}<.001$ ), abnormal DRE ( $\mathrm{OR}=2.47,95 \% \mathrm{CI}=2.03$ to $3.01, \mathrm{P}<.001$ ), positive family history of prostate cancer $(\mathrm{OR}=1.31,95 \%$ $\mathrm{CI}=1.11$ to $1.55, \mathrm{P}=.002)$ and no previous negative prostate biopsy ( $\mathrm{OR}=1.56,95 \% \mathrm{CI}=1.28$ to 1.89 ) were associated with higher risk of prostate cancer. The same variables minus family history plus age and African American race were associated with increased risk of high grade disease. The multivariable PCPT prostate cancer risk equation has been validated in three heterogeneous multi-center studies in the United States with area underneath the receiver operating characteristic curves (AUCs) $66 \%(\mathrm{~N}=446), 66 \%(\mathrm{~N}=4,672)$ and $67 \%(\mathrm{~N}=1,108)$ and the high-grade risk calculator in the latter two studies (AUCs $=63 \%$ and $74 \%$, respectively).

Conclusion: The PCPT risk calculators for prostate cancer and high-grade disease are available online for clinical practice and further validation in different populations. 
S22_A3

STATISTICAL PREDICTION MODELS AND MEDICAL DECISION MAKING

Kattan Michael W.

Cleveland Clinic, Department of Quantitative Health Sciences, 9500 Euclid Avenue / Wb-4, Cleveland, Ohio 44195, USA

Quantitative approaches to medical decision making typically invoke the decision analytic framework. Central to this framework are probabilities and patient preferences. More accurate predicted probabilities should lead to improved medical decision making. However, predicted probabilities can come from a variety of sources, with varying degrees of accuracy. The accuracies of various methods for determining predicted probabilities will be discussed and compared in case studies. These methods include data-driven approaches as well as clinician judgment. As a general rule, it seems that traditional regression models, with special attention paid to predictive accuracy, tend to provide the most accurate predictions presently available. Approaches for implementing them in standalone software are illustrated, as are the use of these models in clinical trial design and analysis.

S22_A4

\section{THE ROC CURVE AND THE BRIER SCORE FOR EVALUATING SINGLE TUMOR MARKERS AND COMPLEX RISK PREDICTION MODELS}

Thomas A. Gerds and Martin Schumacher

Thomas Alexander Gerds, Department of Biostatistics, University of Copenhagen, Øster Farimagsgade 5 B P.O.B. 2099, DK-1014 Copenhagen K, Denmark

A risk prediction model in oncology describes the patient's particular condition so appropriate recommendation regarding treatment can be made based on potential risk. Quite often these models are based on data collected from a group of individuals. The process of developing a risk prediction model may involve many steps of statistical model building such as the assessment of a single tumor marker, the selection of the most important features, and data-driven recognition of complex interactions. Here we discuss and compare two powerful statistical tools for developing risk prediction models: the ROC curve and the Brier score. With a focus on survival response variables we discuss benchmark values, and modifications of these tools for the assessment of complex models that incorporate many features and potential interactions, for example with high dimensional micro array expression data. The methods are illustrated by means of worked examples using data from prognostic factor studies in oncology. 


\title{
MULTIPLE MARKER EVALUATION FOR PANCREATIC CANCER DIAGNOSIS
}

\author{
Volker Heinemann $^{1}$, Dorothea Nagel ${ }^{2}$, Petra Stieber ${ }^{2}$ \\ Med.Department III', Institute of Clinical Chemistry², Klinikum der Universität München - Großhadern, \\ Marchioninistraße 15, 81377 München, Germany
}

Aims: To evaluate the gain in pancreatic cancer diagnosis by combining multiple tumor markers.

Methods:

Levels of CEA, CA19-9, CA72-4, CYFRA21-1, BHCG, NSE, CA125, CA15-3, AFP (Elecsys Roche), and CA 242 (Canag Elisa) from 111 patients with pancreatic cancer, 270 patients with various benign gastrointestinal diseases at time of diagnosis before first treatment and 250 healthy controls were investigated.

The area underneath receiver operating characteristic curves (AUC) were calculated for each marker. For multivariate analysis markers were transformed by fractional polynomial-exponential functions and optimally combined by logistic regression (LR). Age and gender were also considered as potential predictors. Classification rule was externally evaluated by 5 -fold cross-validation and compared by the average area underneath the receiver-operating characteristic curve (AUC) with standard deviation (SD).

Results:

The strongest correlation was between CA19-9 and CA242. The markers AFP and NSE had no discriminating power for pancreatic cancer. As compared to healthy individuals CA 72-4 (AUC $=0.65)$ performed slightly better, all other markers had AUC $>0.70$ up to 0.92 for CA 19-9. As compared to the benign control group CA 15-3, BHCG, CA 125 and CEA had AUC $<0.70$, CA 19-9 had the best discriminative power (0.86).

Statistically significant markers in the multiple LR (healthy individuals) included CA 19-9, CA 125 and CYFRA21-1, AGE was a significant covariate; five fold cross validated AUC (SD) was $0.96(0.008)$.

In benign diseases CA 19-9 and CA 72-4 as well as AGE were significant predictors; five fold cross validated AUC (SD) was $0.88(0.047)$.

Conclusions: Due to the high discriminating power of CA 19-9 alone the combination of multiple markers leads only to a moderate increase of diagnostic capacity for pancreatic cancer. CA 19-9 could be replaced by CA 242 in multivariate analysis.

S22_A6

\section{PREDICTIVE AND PROGNOSTIC SERUM MARKERS IN ADVANCED LUNG CANCER}

$\underline{\text { Stefan Holdenrieder }}{ }^{1}$, Dorothea Nagel ${ }^{1}$, Joachim von Pawel ${ }^{2}$, Hannelore Raith ${ }^{2}$, Knut Feldmann², Ulf-Hakan Stenman ${ }^{3}$, Petra Stieber ${ }^{1}$

${ }^{1}$ University Hospital Munich, ${ }^{2}$ Asklepios Hospital Gauting, Germany; ${ }^{3}$ University Central Hospital Helsinki, Finland

Aim: Nowadays, cancer patients often have various therapeutic options and receive several therapies during disease course. Therefore, pretherapeutic markers are required that are indicative for overall survival and that are able to predict the response of specific therapies.

Methods: In a prospective study, 60 clinical factors, classical and oncological serum markers were analyzed in 311 patients with advanced non-small cell lung cancer undergoing first-line chemotherapy. In uni- and multivariate analyses, they were tested on their prognostic relevance as well as on their ability to predict the response of chemotherapy. In addition, therapeutic efficacy was estimated by lung and apoptotic marker courses during initial chemotherapy.

Results: Clinical factors performance score, weight loss, metastases and chemotherapy showed independent prognostic relevance. When serum markers were tested with these clinical factors, WBC, CRP, albumin, cholesterol, GGT, AP, LDH, CYFRA 21-1, nucleosomes, CA 125, CA 72-4, CA 15-3, CA 19-9, S100, HCGß, and TATI were found to be prognostically significant. A subgroup of these markers was relevant for the prediction of therapy response, too, in particular WBC, CRP, albumin, CYFRA 21-1, nucleosomes, CA 125, CA 72-4, and CA 15-3. Concerning the marker courses, CYFRA 21-1 and nucleosomes both at day 8 and before start of the second cycle of chemotherapy were highly valuable for estimation of therapy response and prognosis.

Conclusion: Pretherapeutic serum markers are valuable tools for the estimation of prognosis and therapeutic efficacy independent from clinical variables and are completed by CYFRA 21-1 and nucleosomes courses during initial chemotherapy. Multivariate models of indepen-dent markers are able to improve current therapy stratification by clinical variables alone. 


\title{
PATTERN OF RELEASE OF VARIOUS ONCOLOGICAL BIOMARKERS IMPROVES THE DIAGNOSTIC CAPACITY OF LUNG CANCER
}

\author{
Petra Stieber ${ }^{1}$, Christine Gruber ${ }^{1}$, Florian Blankenburg ${ }^{1}$, Dorothea Nagel ${ }^{1}$, Rudolf Hatz ${ }^{2}$ \\ ${ }^{1}$ Institute of Clinical Chemistry, University of Munich, Klinikum Grosshadern, Germany; ${ }^{2}$ Surgical Department, \\ University of Munich, Klinikum Grosshadern, Germany
}

${ }^{1}$ Klinikum der Universität München - Großhadern, Marchioninistraße 15, 81377 München, Germany

Aim:

To evaluate the gain in lung cancer diagnosis by combining multiple tumor markers.

Material and Methods:

Levels of CEA, CA19-9, CA72-4, CYFRA21-1, BHCG, NSE, CA125, CA15-3, AFP, TPSA (Elecsys Roche), ProGRP (ALSI Elisa) and SCC (Abbott, AxSym) from 629 patients with lung cancer (85 UICC I, 46 UICC II, 73 UICC III, 53 UICC IV in NSCLC; 64\% LD and 36\% ED in SCLC ) at time of diagnosis before first treatment and of 152 patients with benign lung diseases were assessed. Area underneath receiver operating characteristic curves (AUC) and sensitivities for fixed cut-offs obtaining 95\% specificity were calculated for each marker. Tumor marker values were transformed by fractional polynomial-exponential functions and optimally combined by logistic regression (LR). Classification rule was externally evaluated by 5 -fold cross-validation and the average area underneath the receiver-operating characteristic curve (AUC) with standard deviation (SD), and sensitivity corresponding to the predicted probability cut-off obtaining $95 \%$ specificity was calculated.

Results:

The markers AFP and BHCG had no discriminating power for lung cancer and CYFRA21-1 (0.82) and CEA (0.73) had the highest AUC with $36 \%$ sensitivity at $95 \%$ specificity for benign lung diseases each. In adenocarcinomas CEA and CYFRA 21-1 revealed the best results with $52 \%$ and $25 \%$ sensitivity (AUC 0.79 and 0.81 ). In squamous cell lung cancer CYFRA 21-1 (41\%) and SCC (34\%) reached the highest discriminative power (AUC 0.86, 0.75 respectively). In large cell cancer CYFRA 21-1 (58\%, AUC 0,87) was superior to CEA (46\%, AUC 0.77), in SCLC NSE (63\% and AUC 0.86) and ProGRP (69\% sensitivity, 0.83 AUC) were the best markers.

In multivariate analysis LR obtained in lung cancer in general an AUC (SD) of $0.86(0.02)$ and a sensitivity of 39\% at $95 \%$ specificity, statistically significant markers in the multiple LR included CEA, CYFRA21-1, NSE and age.

Conclusion:

Combination of the tumor markers CEA, CYFRA21-1 and NSE leads to considerable improvement of diagnosis of lung cancer over any single marker. 
WORKSHOP 
NEW ANTIBODIES TO SCCA: SECOND TD-10 REPORT

Kjell Nustad, Tone Varaas, Kari Hauge Olsen, and David J. Warren

Central laboratory, Norwegian Radium Hospital, Montebello, 0310 Oslo, Norway

Aims: Characterization of eight new antibodies to Squamous Cell Carcinoma Antigens (SCCA1 and SCCA2). The antibodies were characterized using as references antibodies: F1H3, F2H7, SCC104, SCC107, K134, K135, and K122.

Methods: Relative affinities were measured using radioiodinated recombinant antigens (rSCCA1 and rSCCA2). Crossinhibition studies were performed using the same radioiodinated preparations. Finally all possible assay combinations were tested using rSCCA1 and rSCCA2 as analytes.

Results: Four antibodies $(374,376,381$, and 380) reacted with both rSCCA1 and rSCCA2. They were separated into three epitope groups consisting of: 374 and 376, 381, and 380. The antibodies 374, 376 and 381 are related. They show cross-inhibition, and can not be combined in assay combinations. Two antibodies (378, and 379) were rSCCA1 specific. They did not show cross-inhibition, but could not be combined for measurement of rSCCA1. Two antibodies (375 and 377) were rSCCA2 specific. They were of the same epitope group and could not be combined for rSCCA2 measurement.

Conclusions: We have added new interesting antibodies to those already characterized. Both specific assays and assays that determine all forms of SCCA1 and SCCA2 can now be made. Only clinical evaluation can determine which assay combination is most useful.

References: Nustad K. et al., Characterization of monoclonal antibodies directed against squamous cell carcinoma antigens: report of the TD-10 workshop. Tumor Biol. 2004; 25: 69-90 
FREE COMMUNICATIONS 


\section{FC1_A1}

\section{A NOVEL MULTIPLE BIOMARKER ASSAY FOR THE DETECTION OF OVARIAN CARCINOMA}

Richard G. Moore, Amy K. Brown, M. Craig Miller, Steve Skates, Thorsten Verch, W. Jeffrey Allard, C.O. Granai, Robert Bast.

Objectives: Multiple studies have demonstrated that women who are cared for at centers with expertise in ovarian cancer have better surgical outcomes and survival. An accurate test is needed to triage patients with a pelvic mass to centers with expertise in treating ovarian cancer patients. This project examined nine novel tumor markers to develop a multiple biomarker assay to predict the risk of ovarian cancer in patients with an ovarian cyst or pelvic mass.

Methods: This was an IRB approved prospective trial. After obtaining informed consent, serum samples were obtained preoperatively from women undergoing surgery for an adnexal mass and analyzed for levels of CA125, SMRP, HE4, CA72-4, osteopontin, activin, inhibin, EGFR and HER2. All pathology results were compared to the tumor markers. Sensitivities at a set specificity of $95 \%$ were determined using logistic regression for each marker individually and all combinations of markers. The best combination of markers was identified and an algorithm was created to categorize patients into risk groups.

Results: 259 patients with adnexal masses were enrolled onto study with 233 patients eligible for analysis; 67 with epithelial ovarian cancers and 166 with benign ovarian neoplasms. The median age for patients with benign tumors was 53 years of age (range 20 to 85) and for patients with malignant tumors 63 years of age (range 18 to 89 ). The most common benign neoplasms were cystadenoma and cystadenofibroma, which together accounted for $27 \%$ of the women with benign disease. Of the malignancies, there were $48(71.6 \%)$ serous tumors, $5(7.5 \%)$ endometrioid, $5(7.5 \%)$ mixed epithelial, and $3(4.5 \%)$ mucinous tumors and $6(8.9 \%)$ other epithelial ovarian malignancies. In patients with epithelial ovarian cancer, $13(19 \%)$ were diagnosed with surgical stage I disease, 2 (3\%) with stage II disease, $46(69 \%)$ with stage III disease, and $6(9 \%)$ with stage IV disease.

The median values for all tumor markers tested differed significantly between subjects with benign masses and cancer. As a single marker, serum CA125 had a sensitivity of $42.0 \%$ at the specificity of $95 \%$. HE4 had the highest sensitivities for detecting a malignancy at the specificity of $95 \%$ with a sensitivity of $71.3 \%$. The combination of HE 4 and CA125 yielded the highest sensitivity relative to any other dual combination of markers, with a sensitivity of $76.8 \%$ at a specificity of $95 \%$. The combination of CA125, HE4, and CA72-4 and that of CA125, HE4, and osteopontin added minimally to the combination of HE4 and CA125. Analysis of patients with stage I ovarian cancer compared with those with benign neoplasms revealed that the serum levels of HE4, CA125, and CA72-4 were significantly different between the two groups. HE4 alone had the highest sensitivity; $49.2 \%$, with a specificity of $95 \%$. The addition of CA125 or any other biomarker combination did not improve the sensitivity of HE4 alone for detection of stage I disease. The dual marker combination of HE4 and CA125 was used to create an algorithm to place patients into low, moderate and high risk groups.

Conclusions: The dual marker combination of HE4 and CA125 can be used in an algorithm to stratify patients into risk groups. These risk assessment can then be used for the triage of patients to tertiary care centers for surgical management. 
FC1_A2

\title{
PROPERTIES OF MELANOSOMES AND THEIR EXPLOITATION IN MELANOMA DIAGNOSIS AND TREATMENT
}

Jan Borovanský

Department of Biochemistry \& Experimental Oncology, $1^{\text {st }}$ Faculty of Medicine, Charles University, U nemocnice 5, 12853 Prague 2, Czech Republic

Expression of specific enzymes (tyrosinase and related proteins) catalysing the process of melanogenesis in subcellular organelles (melanosomes) contributes to a biochemical specificity for melanoma cells. Our studies have shown that melanosomes isolated from various human and animal melanomas represented $19-43 \%$ of the freeze-dried melanoma weight. The presence of melanin in melanosomes and a remarkable amount of melanosomes in melanoma cells endow them with specific properties which can be exploited in practice:

1) During melanogenesis toxic melanin precursors (quinones and semiquinones) and other free radical species are produced which are physiologically compartmentalized in melanosomes where they are converted into insoluble melanin biopolymer or detoxified. We demonstrated that frequent occurence of aberrant melanosomes with membrane defects was typical of melanoma cells. The leakage of toxic compounds (often in combination with a suppression of physiologic scavenging mechanisms) can be exploited in metabolically- based melanoma therapy.

2) Melanosomes behave as an energy-converting device: Photon/heat conversion is used in laser surgery and if the light energy input is too high, vapourization of melanosomes can occur or overproduction of superoxide anions follows with a subsequent formation of toxic hydrogen peroxide.

3) Melanin inside melanosomes has cation exchange properties. Accumulation of various metals, especially zinc, has been repeatedly observed.

4) Melanosomes have affinity for various polycyclic compounds (e.g.methylene blue, phenothiazines, thiourea). Polycyclic compounds labelled with radioactive nuclides have been tested both as melanoma seekers and therapeutic agents.

5) Even non-pigmented melanoma cells can be targetted with antibodies against melanosome-specific proteins (e.g.Pmel17) because these are often expressed also on melanoma cell membranes.

Acknowledgements. Funded by the Czech NRP-MSM(VZ)0021620808.

\section{FC1_A3}

\section{SELECTIVE SUPPRESSION OF HUMAN PROSTATE TUMOR GROWTH IN MICE BY A RECOMBINANT ANTI-PSMA IMMUNOTOXIN}

\author{
Philipp Wolf, Karen Alt, Patrick Bühler, Ulrich Wetterauer, Ursula Elsässer-Beile \\ Department of Urology, University of Freiburg, Breisacher Str. 117, 79106 Freiburg, Germany
}

\begin{abstract}
Aims: There is a distinct medical need for new treatment strategies in patients with prostate cancer, particularly for those in a minimal disease state following primary therapies or with tumor progression despite androgen ablation. Prostate Specific Membrane Antigen (PSMA), a integral type II membrane glycoprotein, is abundantly expressed on prostate cancer epithelial cells and upregulated in androgen insensitive and metastatic disease. Therefore specific immunotherapy against this antigen may be a novel therapeutic option for the management of this tumor.

Materials and Methods: We constructed a recombinant immunotoxin, called A5-PE40, consisting of A5, a single-chain antibody fragment (scFv) against PSMA as binding domain and PE40, a truncated form of Pseudomonas Exotoxin A, as toxin. In vitro cytotoxicity of A5-PE40 was tested on the PSMA expressing, androgen-independent human prostate cancer cell line C4-2 via WST assays. In vivo antitumor activity of the immunotoxin was examined in a C4-2 SCID mouse xenograft model. Mice bearing tumors of non-PSMA expressing DU145 cells were used as controls. Apoptotic tumor cells in histological sections were detected by TUNEL assays.

Results: In vitro a specific cytotoxicity of A5-PE40 on C4-2 cells could be measured with an $\mathrm{IC}_{50}$-value of $220 \mathrm{pM}$. Treatment of SCID mice bearing C4-2 tumors caused a significant inhibition of tumor growth, whereas DU145 xenografts remained totally unaffected. Histological experiments indicated a direct induction of apoptosis in C4-2 xenografts.

Conclusions: A5-PE40 shows potent antitumor activity in mouse xenografts derived from androgen-independent human prostate carcinoma cells. Therefore it merits further development as a molecularly targeted therapeutic agent against prostate cancer.
\end{abstract}


FC1_A4

AUTOANTIBODIES TO ANNEXINS AND CYTOKERATINS

$\underline{\text { Kelly Woodard }}^{1}$, John Robertson ${ }^{1}$, Andrea Murray ${ }^{2}$, Caroline Chapman ${ }^{1}$

Tumor Immunology Group, Division of Breast Surgery, Nottingham City Hospital, Nottingham, UK, NG9 1PB ${ }^{1}$ Oncimmune Ltd, Nottingham City Hospital, UK, NG9 1PB', UK

\begin{abstract}
Aim: There is a wealth of evidence suggesting that tumor-associated antigens elicit a humoral immune response early on in tumor development. Detection of autoantibodies to these antigens may therefore highlight early amplification of the carcinogenic signal.

We have previously shown reproducible detection of autoantibodies to p53, c-myc, HER2, NY-ESO-1, BRCA1, BRCA2 and MUC1 antigens in patients with PBC and DCIS ${ }^{1}$.

Both the annexin and cytokeratin protein families are involved in mechanical stability of cells, and aberrant expression and post-translational modifications in members of both the protein families have been reported in cancer.

The aim of this study was to assess the presence and clinical utility of autoantibodies to annexins I, II \& XI-A, and cytokeratins $8,18 \& 20$ in breast cancer.

Method: cDNAs encoding annexins AI, AII \& AXI-A and cytokeratins 8, 18 \& 20 were cloned and expressed as $6 \mathrm{x}$ histidine tag fusion proteins in $\underline{\text { E.Coli }}$ and purified using immobilised metal affinity chromatography. Recombinant antigens were then used to measure levels of corresponding circulating autoantibodies in breast cancer patients and agematched controls by ELISA.

Results/Conclusions: Preliminary investigations carried out using advanced breast cancer samples showed elevated levels of autoantibodies in breast cancer patients when compared to normal controls. The overall sensitivity and specificity obtained warrants further investigation, and to fully assess the clinical utility of autoantibody detection, current work is ongoing to measure serum autoantibody levels in a larger group of patients with primary breast cancer, the results of which will be presented.
\end{abstract}

Chapman, C., Murray, A., Chakrabarti, J., Thorpe, A., Woolston, C., Sahin, U., Barnes, A., \& Robertson, J. (2007) Autoantibodies in breast cancer: their use as an aid to early diagnosis. Annals of Oncology 18(5): 868-873 
FC2_A1

\section{EARLY DETECTION OF METASTATIC BREAST CANCER DISEASE BY USING TUMORMARKERS}

$\underline{\text { Irene Ertl }}^{1}$, Volker Heinemann ${ }^{2}$, Ingo Bauerfeind ${ }^{3}$, Dorit Lässig ${ }^{2}$, Dorothea Nagel ${ }^{1}$, Dietrich Seidel ${ }^{1}$, Petra Stieber ${ }^{1}$

${ }^{1}$ Institute of Clinical Chemistry , Klinikum der Universität München -Großhadern-, Marchioninistraße 15, 81377

München, Germany

${ }^{2}$ Medical Department III, Klinikum der Universität München-Großhadern-

${ }^{3}$ Gynecological Department, Klinikum der Universität München -Großhadern-

Aims:

Since 1998 we monitor in a prospective non-randomized trial in intervals of 6 weeks breast cancer patients with the aim of early detection of metastatic disease by using CEA and CA 15-3.

Method:

Among the 516 patients participating in our trial we analysed the clinical data of those patients who developed distant metastases $(n=42)$. A reproducible increase of CEA(Abbott, AxSYM) or CA 15-3 (Roche, Elecsys) >=100\%, corresponding to a specificity of $100 \%$, was the indicator for metastatic disease.

Results:

42 patients developed distant metastases. 25 patients (60\%, hereof $83 \%$ hormone receptor positive and $47 \%$ HER2 positive primary tumors) showed the previously defined increase of CEA or CA 15-3 at the time of first distant metastasis (true-positive), 17 patients (40\%, hereof 67\% hormone receptor positive, 25\% HER2 positive) did not (false-negative). At further progressions, an increase of markers was determined in 7 of the 17 false-negative patients (remaining 23\% false-negative).

The marker-positive patients showed a median disease-free interval until recurrence of 49 months (range 14-198). In 15 patients CA 15-3, in 7 CEA, and in 3 CEA and CA 15-3 increased, after a median time of 10 months (range 1.5-30).

All marker-positive patients were asymptomatic at the time of recurrence. 14 of the true-positive patients (56\%) suffered from metastases in one site ( 7 liver, 4 bone, 2 lymph node, 1 lung), 7 in two sites and 4 of multiple metastases. None of the patients with oligometastases became progressive at another site within the following six months and all received treatment within one month after recurrence.

Conclusions:

All marker positive patients were asymptomatic at the time of detection of metastatic disease, $56 \%$ showed metastases in only one site. Whether an organ defined specific treatment can lead more frequently to complete remission of the patients must be shown.

FC2_A2

\section{AFAMIN A NOVEL BIOMARKER FOR THE DIAGNOSIS OF PRIMARY TESTICULAR GERM CELL CANCER - PRIMARY RESULTS}

Benjamin Dieplinger, ${ }^{1,2}$ Hannes Steiner, ${ }^{3}$ Arno Lingenhel, ${ }^{2}$ Ina Graze, ${ }^{4}$ Kurt Kofler, ${ }^{3}$ Jakob Dieplinger, ${ }^{2}$ Wolfgang Loidl, ${ }^{5}$ Günter Janetschek, ${ }^{6}$ Karl Scheiber, ${ }^{7}$ Helmut Klocker, ${ }^{3}$ Florian Kronenberg, ${ }^{2}$ Thomas Mueller, ${ }^{1}$ Meinhard Haltmayer, ${ }^{1}$ Hans Dieplinger ${ }^{2,4}$

${ }^{1}$ Department of Laboratory Medicine, Konventhospital Barmherzige Brueder Linz, Linz, Austria, Seilerstaette 2-4, A-4020 Linz, Austria; ${ }^{2}$ Division of Genetic Epidemiology, Department of Medical Genetics, Molecular and Clinical Pharmacology, Innsbruck Medical University, Innsbruck, Austria; ${ }^{3}$ Department of Urology, Medical University Innsbruck, Innsbruck, Austria; ${ }^{4}$ Vitateq Biotechnology GmbH, Innsbruck, Austria; ${ }^{5}$ Department of Urology, Barmherzige Schwestern Hospital Linz, Linz, Austria; ${ }^{6}$ Department of Urology, Elisabethinen Hospital, Linz, Austria; ${ }^{7}$ Department of Urology, District Hospital Hall/Tyrol, Austria

Background: The aim of the present study was to assess the utility of afamin, a novel glycoprotein with vitamin E binding properties, for the diagnosis of primary testicular germ cell cancer.

Methods: This multi center prospective phase III diagnostic study comprised 64 male patients with suspected testicular tumor. The diagnosis of primary testicular germ cell cancer was based on histological findings after surgical exploration and was classified as seminoma and non-seminoma. Afamin plasma concentrations were measured by sandwich ELISA. Results: Of the 64 patients 23 had a seminoma, 29 a non-seminoma, and 12 had testicular tumor attributable to other 
reasons. Median afamin plasma concentrations were $45.5 \mathrm{mg} / \mathrm{L}$ (range 16.4-84.6) in the seminoma group, $52.4 \mathrm{mg} /$ L (range 14.0-86.4) in the non-seminoma group and $67.6 \mathrm{mg} / \mathrm{L}$ (range 42.3-92.8) in patients without testicular germ cell cancer. Kruskal-Wallis test with post hoc Nemenyi comparisons revealed no significant difference of afamin concentrations between the seminoma and the non-seminoma group. However, both groups showed significantly lower afamin concentrations than the patients without testicular germ cell cancer $(p<0.01$ for seminoma and $p<0.02$ for nonseminoma). Comparing the patients with testicular germ cell cancer $(n=52)$ with the patients without testicular germ cell cancer $(n=12)$ the area under curve for afamin was $0.81(\mathrm{SE}=0.08 ; 95 \% \mathrm{CI}, 0.69$ to 0.90$)$, and the cut off concentration with the highest diagnostic accuracy was $62 \mathrm{mg} / \mathrm{L}$ (sensitivity 79\%, specificity 75\%, diagnostic accuracy $78 \%$ ). In the setting evaluated, diagnostic information obtained by afamin measurements was at least comparable to alphafetoprotein (AFP), ß-human chorionic gonadotrophin (ß-HCG) and lactate dehydrogenase (LDH).

Conclusions: Afamin measurements may be useful as an aid in the diagnosis of testicular germ cell cancer. In contrast to tumor markers commonly used, the diagnostic value of afamin appears to be similar in seminoma and non-seminoma patients.

FC2_A3

\section{AFAMIN AND APOLIPOPROTEIN A-IV - NOVEL PROTEIN MARKERS FOR OVARIAN CANCER}

$\underline{\text { Hans Dieplinger }}^{1,2}$, Alexander Burges ${ }^{3}$, Benjamin Dieplinger ${ }^{2,4}$, Arno Lingenhel ${ }^{2}$, Ina Graze ${ }^{1,2}$, Paul Lüth ${ }^{1,2}$, Günter Daxenbichler ${ }^{5}$, Petra Stieber ${ }^{6}$

${ }^{1}$ Vitateq Biotechnology GmbH, Innsbruck, Austria

${ }^{2}$ Division of Genetic Epidemiology, Department of Medical Genetics, Molecular and Clinical Pharmacology,

Innsbruck Medical University, Innsbruck, Austria

${ }^{3}$ Department of Gynecology, Klinikum Grosshadern, München, Germany

${ }^{4}$ Department of Laboratory Medicine, Konventhospital Barmherzige Brüder, Linz, Austria

${ }^{5}$ Department of Gynecology, Innsbruck Medical University, Innsbruck, Austria

${ }^{6}$ Institute of Clinical Chemistry, Klinikum Grosshadern, München, Germany

Background/Aims:

Comparative proteomics identified the vitamin E binding plasma protein afamin as potential novel tumor marker for OC. For further validation, afamin plasma concentrations were measured in a pilot case/control study. Afamin was significantly decreased in preoperative OC patients compared to controls and increased to normal control values after successful tumor removal. In previous pilot studies we also observed decreased plasma concentrations of apolipoprotein A-IV (apoA-IV, a major plasma protein of the lipoprotein family) in preoperative OC patients. The aim of this study was to evaluate the diagnostic utility of afamin and apoA-IV in OC patients.

Method:

We measured afamin and apoA-IV by specific sandwich-type ELISA using affinity-purified polyclonal and monoclonal antibodies in 90 OC patients, 400 patients with benign gynaecological diseases including endometriosis and 250 controls and compared the results with CA 125 (Elecsys, Roche).

Results:

Afamin values in patients with benign gynaecological diseases did not differ from controls (65.4 mg/l, range 20.2-207 vs. $70.9 \mathrm{mg} / \mathrm{l}$, range 33.4-126) whereas the median was significantly lower in OC patients (53.4 mg/l, range 4.7-92.7). Similar results were obtained with apoA-IV: OC: $8.3 \mathrm{mg} / \mathrm{dl}$, range $0.3-29.5$; benign: 11.7 , range 2.0-32.3; controls: 14.0 , range 5.5-34.0. The corresponding sensitivity at $95 \%$ specificity was $38 \%$ for afamin, $46 \%$ for apoA-IV, $87 \%$ for CA 125 . If CA125 values were $>50 \mathrm{U} / \mathrm{ml}$ we found a correlation between afamin and apoA-IV levels: if afamin was $<40 \mathrm{mg} / \mathrm{l}$ and apoA-IV $<8 \mathrm{mg} / \mathrm{dl}, 18$ of 20 patients suffered from ovarian cancer, if afamin was $>60 \mathrm{mg} / \mathrm{l} \mathrm{and} \mathrm{apoA-IV}>12 \mathrm{mg} / \mathrm{dl}$, 12 of 13 patients had a benign disease.

Discussion:

These data clearly indicate that afamin and apoA-IV alone will not be suitable as screening marker for OC, but in addition to CA125 a much better discrimination between OC patients and benign diseases will be achieved. 
FC2_A4

\title{
PET IMAGING WITH CU-64 LABELED HUMANIZED ANTI-CEA ANTIBODY
}

John E. Shively ${ }^{1}$, James Bading ${ }^{2}$, David Colcher ${ }^{2}$, Andrew Raubitschek ${ }^{2}$.

${ }^{1}$ Beckman Research Institute of the City of Hope, Duarte, CA 91010, USA.

${ }^{2}$ City of Hope, Duarte CA 91010, USA.

Humanized anti-CEA antibody hT84.66-M5A (M5A) has an affinity constant $>10^{10} \mathrm{M}^{-1}$, no reactivity with CEA crossreacting antigens found in normal tissues, and $>90 \%$ human protein sequence. We developed a novel bifunctional chelator, DO3A-VS (1, 4, 7-tris(carboxymethyl)-10-(vinylsulfone)-1, 4, 7, 10-tetraazacyclododecane) that binds a range of radiometals including ${ }^{111} \mathrm{In}$ for gamma-ray imaging and ${ }^{64} \mathrm{Cu}$ for Positron Emission Tomography (PET), and which can be conjugated with negligible loss of immunoreactivity either to sulfhydryls $(\mathrm{SH})$ in the hinge region of lightly reduced immunoglobulins or surface lysines $(\mathrm{NH})$ of immunoglobulins.

Methods: Athymic mice peripherally xenografted with CEA-positive human colon tumors (LS-174T) were injected with ${ }^{111}$ In-labeled or ${ }^{64} \mathrm{Cu}$-labeled SH-DO3A-VS-M5A, NH-DO3A-VS-M5A, or DOTA-M5A and sacrificed at various time points for biodistribution measurements. Other mice injected with ${ }^{64} \mathrm{Cu}-$ labeled SH-DO3A-VS-M5A or NH-DO3A-VS-M5A were imaged serially with small animal PET from 1 to $48 \mathrm{~h}$ post injection and then sacrificed for biodistribution measurements.

Results: Virtually identical biodistributions were obtained for SH- and NH-DO3A-VS-M5A or DOTA-M5A whether radiolabeled with ${ }^{111} \mathrm{In}$ or ${ }^{64} \mathrm{Cu}$. Rapid tumor uptake of radiolabel was observed, reaching $40 \%$ injected dose/gram or more by $48 \mathrm{~h}$. Importantly, excellent PET images of tumor were obtained as early as $22 \mathrm{~h}$ after injection of ${ }^{64} \mathrm{Cu}-\mathrm{labeled} \mathrm{SH}-\mathrm{or}$ NH-DO3A-VS-M5A.

Conclusions: Based on our correlative studies comparing the kinetics of radiolabeled anti-CEA antibodies in murine models with those in man, we predict that ${ }^{64} \mathrm{Cu}$-labeled intact, humanized antibodies can be used to image $\mathrm{CEA}$ positive tumors in the clinic.

FC2_A5

\section{ANALYSES OF COMPARATIVE GENOMIC HYBRIDIZATION (CGH) ARRAYS ON URINARY SPECIMENS OF PATIENTS WITH BLADDER CANCER}

Virginia Lopez Martinez ${ }^{1}$, Javier Suela Rubio ${ }^{2}$, Pilar Gonzalez-Peramato ${ }^{3}$, Alvaro Serrano ${ }^{4}$, Juan Cruz Cigudosa Garcia $^{2}$ and Marta Sanchez-Carbayo ${ }^{1^{*}}$

${ }^{1}$ Spanish National Cancer Research Center, Tumor Markers Group, Madrid, Spain; ${ }^{2}$ Spanish National Cancer Research Center, Molecular Cytogenetics Group, Madrid, Spain; ${ }^{3}$ Hospital de Guadalajara, Pathology, Guadalajara, Spain; ${ }^{4}$ Hospital de Guadalajara, Urology, Guadalajara, Spain

\begin{abstract}
Aims: The advent of high-throughput technologies is allowing identification of targets and biomarkers of human diseases. CGH arrays represent a comprehensive tool to discover genomic alterations associated to neoplastic progression. Here, we report a novel approach applying CGH arrays to urinary specimens to characterize bladder cancer progression.

Methods: Genomic DNA from urine specimens $(\mathrm{n}=22)$ belonging to patients with urothelial carcinoma (pTa, $\mathrm{pT} 1$, and pT2) and controls (reference pools) was fluorescently labelled and hybridized to G4410B arrays (Agilent). The presence of the disease was confirmed by cystoscopy. Data analyses were performed using the Feature Extraction v9.5, CGH Analytics v3.3, and Insilico GEPAS v3.1 softwares. Validation of critical loci was performed using multiple ligationdependent probe amplification (MLPA) analysis on DNA from the same urinary specimens, bladder cell lines and paired bladder tumors. Moreover, protein expression of certain targets were assessed by immunohistochemistry on tissue arrays containing bladder tumors $(\mathrm{n}=125)$.

Results: Quality control of array-CGH was initially proven to reach optimal reproducibility in dilution experiments and reference pools comparisons. Genomic regions differentially expressed between DNA belonging to patients with bladder cancer and controls identified by array-CGH analysis included 1q, 8p, 15q, 19q, and 20q, among others. MLPA validation confirmed alterations on 20q, among others, using DNA obtained from urine, cell lines and paired bladder tumor specimens. Immunohistochemistry analyses revealed that prefoldin (20q) is highly expressed in bladder tumors as compared to normal urothelium. Prefoldin expression patterns were associated with tumor progression on bladder cancer tissue arrays $(\mathrm{p}<0.05)$.

Conclusions: Array-CGH on urinary specimens was shown to be feasible and provided critical information identifying candidate targets and biomarkers of bladder cancer. Immunohistochemical analyses served to validate the relevance of certain proteins, including prefoldin in bladder cancer progression.
\end{abstract}


FC2_A6

\title{
FREQUENT EPIGENETIC INACTIVATION OF DICKKOPF FAMILY GENES IN GASTROINTESTINAL TUMORS
}

\author{
Hiromu Suzuki ${ }^{1}$, Minoru Toyota ${ }^{1}$, Reo Maruyama ${ }^{1}$, Kohzoh Imai $^{2}$, Yasuhisa Shinomura ${ }^{1}$ \\ ${ }^{1}$ First Department of Internal Medicine, Sapporo Medical University, S1, W16, Chuo-Ku, Sapporo, 060-8543, Japan \\ ${ }^{2}$ President, Sapporo Medical University, S1, W17, Chuo-Ku, Sapporo, 060-8556, Japan
}

Introduction:

Activation of Wnt signaling has been implicated in tumorigenesis, and epigenetic silencing of Wnt antagonist genes has been detected in various cancers. We have previously shown that secreted frizzled-related protein $(S F R P)$ genes are frequently silenced by promoter hypermethylation in colorectal cancer (CRC) and gastric cancer (GC). Our aim in the present study was to characterize the epigenetic alteration of $D I C K K O P F(D K K)$ family genes in gastrointestinal tumors.

Methods:

We analyzed expression status of $D K K$ genes in nine CRC cell lines by RT-PCR. We analyzed methylation status of $D K K$ genes by methylation-specific PCR (MSP) and bisulfite sequencing. A total of 58 primary CRC tumors and 31 primary GC tumors were also analyzed for $D K K$ methylation. We performed functional analysis of DKK genes by colony formation assay.

Result:

We found that all known $D K K$ genes are frequently silenced in CRC cells $(D K K 1,3 / 9,33 \% ; D K K 2,8 / 9,89 \%$; $D K K 3$, $5 / 9,56 \% ; D K K 4,5 / 9,56 \%)$. DKK1, -2 and -3 have 5' $\mathrm{CpG}$ islands, and show an inverse relation between expression and methylation. $D K K$ s also were frequently methylated in primary CRCs $(D K K 1,7 / 58,12 \% ; D K K 2,45 / 58,78 \% ; D K K 3,12 /$ $58,21 \%)$ and $\mathrm{GCs}(D K K 1,15 / 31,48 \% ; D K K 2,26 / 31,84 \% ; D K K 3,12 / 31,39 \%)$. Ectopic expression of DKKs suppressed colony formation of CRC cells.

Conclusion:

Our results demonstrate that $D K K s$ are frequent targets of epigenetic silencing in CRC. Loss of $D K K$ s may be one of the mechanism to activate Wnt pathway in gastrointestinal tumors.

\section{FC2_A7}

\section{LUNG CANCER - CAN AUTOANTIBODIES PROVIDE AN AID TO DIAGNOSIS?}

CJ Chapman; A Murray; J McElveen; U Sahin; U Luxemburger; Ö Türeci; R Wiewrodt; AC Barnes; JFR Robertson.

Tumor Immunology Group, Division of Breast Surgery, Clinical Sciences Building, City Hospital, Nottingham, NG5 $I P B, U K$

Background and Aims: World wide approximately 11 million people are diagnosed with cancer each year and more than 7 million people die of the disease. Early detection and diagnosis of all cancer types before the cancer has spread is essential to improve survival. To achieve this it is necessary to detect the tumor presence at the very earliest stages of carcinogenesis and before the symptomatic stage where physical and diagnostic signs become evident since by this stage, in many patients, the cancer will have already spread. Lung cancer is the largest single cause of deaths in the world (1.1 million annually) since it almost invariable associated with poor prognosis by the time it is identified. At present there is little to offer for early diagnosis, even in individuals at high risk of developing the disease. This study aims to assess the diagnostic potential of tumor-associated autoantibody profiles as an aid to other lung cancer screening modalities.

Materials and Methods: Plasma from normal controls $(\mathrm{n}=50)$, patients with non small cell lung cancer $(\mathrm{NSCLC})$ $(\mathrm{n}=82)$ and patients with small cell lung cancer (SCLC) $(\mathrm{n}=22)$ were investigated for the presence of autoantibodies to p53; c-myc; HER2; NY-ESO-1; CAGE; MUC1 and GBU4-5 by enzyme-linked immunosorbent assay.

Summary: $74 \%$ of all the lung cancer patients showed elevated levels of autoantibodies, with a specificity of $92 \%$. There was no significant difference between the detection rates between node positive and node negative patients or between patients with NSCLC and SCLC. These results suggest that measurement of an autoantibody response to one or more tumor associated antigens in an optimised panel assay, could provide a sensitive and specific blood test for the early detection of lung cancer. 
FC2_A8

\title{
IMMUNOTHERAPY PROLONGS THE SERUM CEA-TPA-CA15.3 LEAD TIME AT THE METASTATIC PROGRESSION IN ENDOCRINE-DEPENDENT BREAST CANCER PATIENTS.
}

\author{
A Nicolini, P Ferrari, L Anselmi, E Dal Canto, M Collareta, *A Carpi, **G Rossi. \\ Department of Internal Medicine, University of Pisa, *Department of Ageing and Reproduction, University of Pisa, \\ **Unit of Epidemiology and Biostatistics, Institute of Clinical Physiology, CNR., Pisa
}

In metastatic breast cancer tumor markers' increase predicts, by a few months (lead time) disease progression. We reported a prolonged clinical benefit and overall survival in 32 breast cancer patients with endocrine dependent metastatic disease and treated with a hormone immunotherapy schedule. In these 32 patients the lead time at the progression of metastatic disease during therapy was compared with that at the onset of metastases when the same patients were without treatment and with that of a control group without immunotherapy. At disease progression, CEATPA-Ca15.3 sensitivity was $96 \%$ in the studied patients and $87 \%$ in the controls. At the progression in the studied patients CEA-TPA-CA15.3 lead time ( $\mathrm{m} \pm \mathrm{sd}$, months) was significantly longer than in controls $(12.5 \pm 13.4 \mathrm{vs} 2.4 \pm 4.2)$ $(\mathrm{P}=0.000)$. Besides, only in studied patients the lead time was significantly longer at the progression than at the metastatic onset $(\mathrm{P}=0.002)$. The CEA-TPA-CA15.3 tumor marker panel accurately predicted metastatic disease progression and immunotherapy significantly prolonged the CEA-TPA-CA15.3 lead time. This can be used for anticipating salvage treatment in these patients.

FC2_A9

\section{CYFRA 21-1 AND CEA AS PROGNOSTIC FACTORS IN OPERATED STAGE I NON SMALL CELL LUNG CANCER PATIENTS?}

Florian Blankenburg ${ }^{1}$, Rudolf Hatz ${ }^{2}$, Dorothea Nagel ${ }^{1}$, Judith Reinmiedl ${ }^{3}$, Dietrich Seidel ${ }^{1}$, Petra Stieber ${ }^{1}$

${ }^{1}$ Institute of Clinical Chemistry, Klinikum der Universität München -Großhadern-, Marchioninistraße 15, 81377 München, Germany

${ }^{2}$ Surgical Department, Klinikum der Universität München -Großhadern-, Germany

${ }^{3}$ Asklepios Lungenfachkliniken, Gauting, Germany

Aims:

To validate the prognostic impact of the preoperative levels of CYFRA 21-1 and CEA and the corresponding Tumor Marker Index (TMI) in stage I NSCLC as described by Muley et al ${ }^{1}$.

Methods:

We retrospectively examined 240 stage I NSCLC patients (171 male, 69 female; 80 pT1, 160 pT2; 95 Squamous-Cell-, 83 Adeno-, 30 Large-Cell-Ca, 32 other histologies), treated between 1986 and 2004, complete resection (R0).

Results:

The overall 3-/5-year survival was $72 \%$ and $58 \%$. In univariate analysis male gender $(\mathrm{p}=0.0009)$ and age $>70$ y $(\mathrm{p}=0.0041)$ were prognostic factors for a worse outcome. There were no significant differences between pT1 and pT2 patients or between the histological subtypes.

The 3-year survival was $68.3 \%$ for CYFRA $21-1>3,3 \mathrm{ng} / \mathrm{ml}$ (Roche, Elecsys) and 73.0\% for lower marker levels; the corresponding 5-year survival rates were 59,2\% and 56,8\% respectively (not significant).

For CEA $>6,7 \mathrm{ng} / \mathrm{ml}$ (Abbott, AxSym) the 3-year survival was $72,5 \%$ and $69,2 \%$ for lower marker levels; the corresponding 5 -year survival rates were $60,6 \%$ and $45,8 \%$ respectively $(\mathrm{p}=0,09)$.

We could not significantly discriminate the 3 prognostic groups by using the TMI as described by Muley.

Conclusions:

Elevated levels of CEA, male gender and age $>70$ correspond with poorer outcome in early stage of NSCLC. As CYFRA 21-1 was not able to indicate a worse outcome in our analysis, we were not able to reproduce the prognostic value of the TMI introduced by Muley. 
POSTERS 


\title{
CELL CYCLE DISTURBANCES AND MITOTIC CATASTROPHES IN CELL LINES FOLLOWING LOW DOSE RATE BETA-IRRADIATION
}

\author{
David Eriksson ${ }^{1)}$, Theres Lindgren ${ }^{1)}$, Per-Olov Löfroth ${ }^{2)}$, Lennart Johansson ${ }^{2)}$, Katrine Åhlström Riklund ${ }^{3)}$, \\ Torgny Stigbrand ${ }^{1)}$ \\ 1) Department of Immunology,Umeå University, SE-901 85 Umeå, Sweden (david.eriksson@climi.umu.se) \\ 2) Department of Radiation Physics, Umeå University, SE-901 85 Umeå, Sweden \\ 3) Department of Diagnostic Radiology, Umeå University, SE-901 85 Umeå, Sweden
}

\begin{abstract}
Aims: Antibodies specific for tumor-antigens and labelled with ${ }^{131} \mathrm{I}$ can deliver low dose, low dose rate irradiation to tumors and cause growth retardation at experimental radioimmunotherapy. The aim was to elucidate the sequential molecular and cellular events occurring in four cell lines exposed to continuous low dose-rate radiation.

Methods: Activation of cell cycle checkpoints and mitotic behaviour was investigated in four cell lines: HeLa Hep2, Jurkat, LS174T and HT29 following low dose irradiation delivered by ${ }^{131} \mathrm{I}$ in the cell culture media. Western blots, FACS analysis and immunofluorescence stainings were performed for detection of mitotic aberrations and apoptotic induction. Results: G2/M arrests were demonstrated by FACS analysis. The G2/M arrest was transient and the cells reentered the cell cycle still containing unrepaired cellular damage. This premature entry caused an increase of anaphase bridges, lagging chromosomal material and multipolar mitotic spindles as visualised by propidium iodide staining and immunofluorescence staining with alpha-tubulin and gamma-tubulin antibodies. Furthermore a dose dependent significant increase in centrosome numbers, as well as a dose dependent increase of polyploid cells were detected. These disturbances caused the cells to progress into mitotic catastrophes and a fraction of these dying cells demonstrated apoptotic features as displayed by TUNEL staining 5-7 days following irradiation.

Conclusion: Low dose, low dose rate irradiation was demonstrated to force all four cell lines into mitotic catastrophe and delayed apoptosis. These phenomenon might be important cell death mechanisms involved in tumor growth retardation following radioimmunotherapy of tumors.
\end{abstract}

\section{P1_2}

\section{ANDROGEN INHIBITS THYMOSIN BETA4 EXPRESSION IN PROSTATE CANCER CELLS}

\author{
Kazuyuki Hirano, Mai Ito, Kazuhiro Iguchi, Shigeyuki Usui
}

Laboratory of Pharmaceutics, Gifu Pharmaceutical University, 5-6-1 Mitahora-higashi, Gifu, Gifu 502-8585, Japan.

\begin{abstract}
Aims: Most patients with advanced prostate cancer will be treated with an androgen ablation therapy because the growth and progression of prostate cancer are initially androgen-dependent. The androgen-ablation therapy is effective treatment for advanced prostate cancer, but the tumor often progresses toward a more aggressive phenotype. The prostate cancer in this state is often difficult to cure and the development of effective treatment strategies is under consideration. We tried to determine the changes in genes associated with the malignant progression, and found that thymosin beta4, which is involved in tumor metastasis, was increased in androgen-sensitive LNCaP cells grown in the androgen-deficient charcoalstripped FCS.

Methods: The mRNA expression of thymosin beta4 was measured by real-time PCR analysis. The transcriptional activity of thymosin beta4 was determined by luciferase assay using reporter plasmid containing 5 '-flanking region of thymosin beta4.

Results: Thymosin beta4 mRNA expression was increased in LNCaP cells in the androgen-deficient condition and decreased by dihydrotestosterone treatment. Androgen receptor antagonist bicalutamide inhibited thymosin beta4 expression in a dose-dependent manner. In androgen receptor-negative PC-3 cells, no significant effects on thymosin beta4 gene expression were observed. Moreover, luciferase assay revealed that the regulation of thymosin beta4 mRNA expression by androgen was due to the transcriptional activation. Deletion analysis demonstrated that the region between $-83 \mathrm{bp}$ and $-46 \mathrm{bp}$ of thymosin beta 4 gene was responsible for the regulation of the transcriptional activity by androgen. Conclusion: Thymosin beta4 expression is negatively controlled at the transcriptional level by androgen.
\end{abstract}


P1_3

\title{
TNF EFFECTS ON HEMATOLOGICAL CELL LINES
}

\author{
Jurisic V, Kojic V, Kraguljac N, Jakimov D, Bogdanovic G, Bumbasirevic V
}

Tumor necrosis factor (TNF)-alpha, a pleiotropic cytokine, has been shown to induce mostly apoptosis in sensitive cells. We investigated the in vitro dose- and time-related effect on 3 different hematological cell lines, including the erythro leukemia, K-562 cells; a malignant B-cell line Raji, derived from Burkitt lymphoma patients; and a novel cell line derived from therapy related myelodisplastic patients. The effects were determined at different intracellular levels. The membrane alteration was estimated by lactate dehydrogenase (LDH) release, by flow cytometry (using annexin V and Propidium Iodide) and on morphology. The intracellular metabolic energy analyzed by the total intracellular enzyme activity after cell sonification, and the total cytosole protein mass by sulforhodamine B assay. The cell growth was estimated by incorporation of [(3)H]thymidine into DNA. Significant increase of LDH through cell membrane alteration was accompanied by a decrease of intracellular metabolized energy and total protein mass. The effect of apoptosis or cell proliferation was different depending on cell type. In addition, TNF-alpha at lower concentrations (125 and $250 \mathrm{pg} / \mathrm{ml})$ significantly induced cell proliferation in Raji cells, compared to $1.000 \mathrm{pg} / \mathrm{ml}$ of TNF-alpha, which induced more cell death. TNF-alpha induced a different rate of apoptosis that depended on cell type and level of TNF receptor molecule expression. We also correlated the rate of apoptosis in respect to TNF receptor 1 (TNFR-1) and TNFR-2 type on these cells and found that TNFR1 is more associated with a high rate of apoptosis while TNFR 2 is more associated with cell proliferation.

\section{P1_4}

\section{FREE AND N-(2-HYDROXYPROPYL)METHACRYLAMIDE (HPMA) BOUND DOXORUBICIN: DIFFERENCES IN GLYCOSYLATION OF EL-4 CELL SURFACE MEMBRANE}

Kovář L., ${ }^{1}$ Etrych T., ${ }^{2}$ Šubr V., ${ }^{2}$ Strohalm J., ${ }^{2}$ Sklenář J., ${ }^{1}$ Chytil P., ${ }^{2}$, Ulbrich K., ${ }^{2}$ and Ŕíhová B. ${ }^{1}$

${ }^{1}$ Institute of Microbiology ASCR v.v.i., ${ }^{2}$ Institute of Macromolecular Chemistry ASCR, v.v.i. Prague, Czech Republic

Aims:

Copolymers of $\mathrm{N}$-(2-hydroxypropyl)methacrylamide (HPMA) have been developed as polymeric carriers of drugs. Conjugates containing aminolytically bound doxorubicin (PK1), in contrast to those with hydrolytically bound doxorubicin (HYD) and the parent drug (Dox), accumulate in intracellular membranes, including those of the Golgi apparatus and endoplasmic reticulum. Therefore, the aim of this study was to determine changes in surface membrane glycosylation of cells after exposure to HPMA conjugates.

Method:

The saccharide composition was determined by flow cytometry by lectin binding or in surface membrane fraction of EL$4 \mathrm{~T}$ cell lymphoma exposed to HPMA copolymers by HPAEC-PAD analysis. Standard western blotting procedure was performed to determine the amount of CD 43, CD44 and CD45 molecules.

Results:

The PK1, in contrast to HYD and free Dox, increased surface membrane glycosylation and also changed oligosaccharide composition. In contrast to overexpressed CD43 molecule in cells exposed to PK1, the expression of CD44 and CD45 was not affected in cells exposed to different forms of doxorubicin. The CD43 is known to possess an anti-adhesive (,barrier") structure and our results also show that EL-4 cells incubated with PK1 partially lose their adhesiveness. The impact of enhanced surface glycosylation and CD43 overexpression is the subject of further studies.

Conclusions:

The EL-4 cell line possesses enhanced and simultaneously changed surface glycosylation when incubated with the PK1 in contrast to controls or cells exposed to HYD or free doxorubicin. This effect can be explained by the overexpression of the CD43 molecule. In our system, this molecule can be considered to have an anti-adhesive function.

\section{ACKNOWLEDGMENTS}

The research was supported by the Grant Agency of the Czech Republic No. 305/05/2268 and by the Institutional Research Concept AV0Z50200510. 


\title{
DIFFERENCES IN REACTIVITIES TO SCCA1 AND SCCA2 MEASURED WITH ARCHITECT SCC AND IMX SCC
}

\author{
$\underline{\text { Katsumichi Takeda }}^{1}$, Akihiro Murakami ${ }^{2}$, Eiji Kobayashi $^{1}$ and Norihiro Sugino ${ }^{2}$ \\ ${ }^{1}$ Research and Development, Abbott Japan, 278 Matsuhidai, Matsudo, Chiba 270-2214, Japan., ${ }^{2}$ Yamaguchi University \\ Graduate School of Medicine, Department of Obstetrics and Gynecology, 1-1-1 Minamikogushi, Ube, Japan
}

Squamous cell carcinoma antigen (SCCA) is a tumor related protein of squamous cell carcinoma of the uterine cervix, lung, esophagus, head and neck, and skin. Two genes are known for SCCA, i.e. SCCA1 and SCCA2, and they are thought to code neutral and acidic fractions of SCCA, respectively. IMx SCC and ARCHITECT SCC, automated immunoassay systems for SCCA, are available as assay kits for the measurement of serum SCCA.

In some clinical specimens, SCCA values measured with ARCHITECT were higher than those with IMx. Therefore, to compare these immunoreactivities in both assays, various ratios of recombinant SCCA1 and recombinant SCCA2-spiked SCCA-free serum were prepared. ARCHITECT showed higher values for SCCA1-spiked specimens than those assayed with IMx. For SCCA2-spiked specimens, however, ARCHITECT showed almost the same values as IMx. ARCHITECT showed higher values than IMx when the SCCA1: SCCA2 ratio was high in SCCA-free serum

These findings suggest that ARCHITECT has higher reactivity to the native SCCA1 in serum than to the SCCA2 and the actual values are dependent on the proportion of SCCA1 in the sample. Furthermore, it appeared that SCCA values in clinical specimens measured with ARCHITECT showed more correct values than those with IMx because ARCHITECT has higher reactivity for total SCCA. The new ARCHITECT assay kit is an excellent tool for measurement of SCCA in clinical specimens.

P1_6

\section{GENOMIC SCREENING FOR GENES SILENCED BY DNA METHYLATION IN MULTIPLE MYELOMA}

Hiroshi Yasui, Minoru Toyota, Reo Maruyama, Isao Tarasawa, Hiromu Suzuki, Toshiaki Hayashi, Hajime Sakai, Tadao Ishida, Hideki Asaoku, Takashi Tokino, Kohzoh Imai, Yasuhisa Shinomura

First Department of Internal Medicine, Sapporo Medical University, South-1, West-16, Chuo-ku, Sapporo, 060-8543, JAPAN

Epigenetic changes such as DNA methylation have recently been shown to play important roles in tumorigenesis in multiple myeloma (MM). However, in most cases, the methylation status has been investigated for just a single gene, and therefore global methylation profile of MM remains undefined. In this study, we performed genomic screening using microarray to identify genes inactivated by DNA methylation in MM, and also examined methylation profiles of MM patients.

We found that 466 genes were upregulated more than two folds by treatment of DNA methyltransferase inhibitor 5Aza-2'-deoxycytidine in both RPMI8226 and KMS12PE cell lines. Next, we performed quantitative RT-PCR and DNA methylation analysis in MM cell lines and then we identified 20 genes which were silenced by DNA methylation. Importantly, methylation profiles of $29 \mathrm{MM}$ patient samples revealed that 10 genes were methylated in a tumor-specific manner, and that the frequencies of methylation of these genes were ranged from $48 \%$ to $7 \%$. Moreover, we observed that methylation of the genes is not a random event, but simultaneous methylation of multiple genes occurs in a subset of cases. Our finding suggests that analysis of methylation profiles in MM patients might be useful for clinical application. 
IGF-I RECEPTOR IS A CANDIDATE MOLECULAR TARGET FOR BOTH HUMAN ESOPHAGEAL SQUAMOUS CELL CARCINOMA AND ADENOCARCINOMA

Yasushi Adachi ${ }^{1,2}$, Yu Wang ${ }^{1}$, Arisa Imsumran ${ }^{1}$, Rong $\mathrm{Li}^{1}$, Hua $\mathrm{Li}^{1}$, Wenhua Piao ${ }^{1}$, Hiroyuki Yamamoto ${ }^{1}$, Yasuo Kato $^{2}$, Choon-Taek Lee ${ }^{3}$, David P. Carbone ${ }^{3}$, Yasuhisa Shinomura ${ }^{1}$, and Kohzoh Imai ${ }^{1}$.

${ }^{1}$ First Department of Internal Medicine, Sapporo Medical University, Sapporo 060-8543, Japan, ${ }^{2}$ Internal Medicine, Sapporo Shirakaba-dai Hospital, Sapporo 062-0052, Japan, ${ }^{3}$ Vanderbilt-Ingram Cancer Center and Departments of Medicine and Cell Biology, Vanderbilt University, Nashville, TN 37232-6838, USA.

$<$ Aims $>$ Targeting therapies against growth factor receptors have been reported and some drugs are in clinical use. Insulin-like growth factor (IGF)-I receptor (IGF-Ir) signaling is required for carcinogenicity and tumor development of many malignancies, but this pathway has not been well studied in esophageal carcinomas. Esophageal cancer has a very poor prognosis. The main types are squamous cell carcinomas (ESCC) and adenocarcinoma (EAC). Previously, we have reported that the IGF-axis may play a key roles in the progression of ESCC and its detection may be useful for the prediction of recurrence/poor-prognosis and possibly for selecting patients for IGF-Ir-targeting therapy. Here, we analyzed the effect of IGF-Ir in both esophageal carcinomas. We also want to develop potential targeted therapeutics and have constructed a recombinant adenovirus expressing dominant negative form of IGF-Ir (Ad-IGF-Ir/dn).

$<$ Methods $>$ We assessed whether IGF-Ir will be a target for both esophageal cancers. Ad-IGF-Ir/dn was used to treat ESCC and EAC lines. We assessed the effect of IGF-Ir/dn on signaling blockade, growth, and apoptosis.

$<$ Results $>$ IGF-BP3 blocked in vitro growth. It is down-regulated in serum-free conditions and is recovered by IGF. IGFIr/dn suppressed proliferation and colony formation in both ESCC and EAC. IGF-Ir/dn up-regulated both stressor- and chemotherapy-induced apoptosis in both cells. It effectively blocked both IGFs-induced Ak activation. IGF-Ir/dn might form at least part of a complex containing ligands in both media and cells, suggesting that bystander effect.

$<$ Conclusions $>$ IGF-axis might play key roles in tumor progression of esophageal cancers. Ad-IGF-Ir/dn may be a useful anticancer therapeutic widely for esophageal carcinomas.

P2_2

\section{NEW PERSPECTIVES IN ELECTROCHEMICAL DETERMINATION OF METALLOTHIONEINS}

Vojtech Adam $^{1}$, Ivo Fabrik ${ }^{1,2}$, Johana Nakielna ${ }^{1}$, Vendula Hrdinova ${ }^{1}$, Pavlina Blahova ${ }^{1}$, Sona Krizkova ${ }^{1}$, Jiri Kukacka ${ }^{5}$, Richard Prusa ${ }^{5}$, Rene Kizek ${ }^{1}$

${ }^{1}$ Department of Chemistry and Biochemistry, Faculty of Agronomy, Mendel University of Agriculture and Forestry, Zemedelska 1, CZ-613 00 Brno, Czech Republic

${ }^{2}$ Department of Biochemistry, Faculty of Science, Masaryk University, Kotlarska 2, CZ-611 37 Brno, Czech Republic ${ }^{3}$ Department of Clinical Biochemistry and Pathobiochemistry, $2^{\text {nd }}$ Faculty of Medicine, Charles University, V Uvalu 84, CZ-150 06 Prague 5, Czech Republic

The success of treatment of the disease depends on many factors such as prevention, early diagnosis and sensitive diagnostics. A group of cysteine-rich metal-binding proteins called metallothioneins can be considered as new tumor markers. The main aim of this work was to determine metallothionein in whole blood, serum and tissue from patients with various cancer types. Electrochemical methods using a mercury electrode coupled with an adsorptive transfer stripping technique are among the most sensitive procedures available to detect metallothionein. The adsorptive technique coupled with differential pulse voltammetry (Brdicka reaction) and/or chronopotentiometric stripping analysis enables us to measure subnanomolar levels of metallothionein in low volumes of a sample (units of $\mu \mathrm{L}$ ). Nevertheless, using this technique to measure hundreds of samples is time consuming. Thus, we attempted to automate the analysis. For these purposes we used an electrochemical instrument, 745 VA Stand (Metrohm, Switzerland), in connection with a VA Trace Analyzer and 695 Autosampler (Metrohm, Switzerland). Blood from sixty patients with cancer were analyzed by both mentioned techniques. The levels of metallothionein determined in blood of the patients varied from 2 to $4 \mu \mathrm{M}$, whereas the results obtained by the mentioned techniques were in good agreement (deviation up to $5 \%$ ). Moreover, we used an enzyme linked immunosorbent assay to evaluate the results obtained by electrochemical methods. Deviation between the content of MTs did not exceed $10 \%$.

Acknowledgment: This work was supported by grants from Beh Terryho Foxe 2007, GAAV IAA 401990701, Liga proti rakovine and VUP 2007. 


\section{URINARY EXCTRETION OF THE TRASFORMING GROWTH FACTOR- $\alpha$ (TGF- $\alpha$ ) AND THE EPIDERMAL GROWTH FACTOR(EGF)OF THE PATIENTS WITH BENIGN OR MALIGNANT DISEASES: APPRAISAL ON CANCEROGENESIS.}

$\underline{1}$ Buda Francesco ${ }^{2}$ Aragona Pietro, ${ }^{3}$ Tagliapietra Antonella, Scopelliti Marcella, Burlandi Mario, ${ }^{4}$ Cammareri Francesco,Logoteta Mario, Pagliuzzi Michelangelo, Pietrobon Mirella.

${ }^{1}$ Medical Oncology and Prevention, City of Udine Hospital, Udine, Italy; ${ }^{2}$ Clinical Research Laboratory and Pathology, S.M.Battuti Hospital, Pordenone, Italy, ${ }^{3}$ Dpt of Research Laboratory and Pathology,University Roma, Italy, ${ }^{4}$ Dpt of Surgery, University Roma, Italy

Purpose: TGF- $\alpha$ is produced by many transformed cells and shows homology to Epidermal Growth Factor(EGF) which is found in human body fluids and is also detectable in urine and effusions of cancer patients.

Methods: We established a radioimmunoassay specific for EGF and TGF- $\alpha$ to study quantitatively EGF and TGF- $\alpha$ in $24 \mathrm{~h}$ urine samples of 120 patients (aged 40 - 75 years, average 62 years) with various malignanciess, compared to 72 persons (aged $45-78$ years, average 64 years) with or without benign disease.

Results: In 3 of 5 patients (60\%) with non neoplastic colon diseases we found TGF- $\alpha$ levels of $>2,5 \mu / \mathrm{g}$ creatinine. In patients with cancer of the gastrointestinal tract,the incidence rates of pathological urinary TGF- $\alpha$ concentrations were as follows: 3 of 8 patients (37,5\%) carcinoma of the esophagus; 4 of 13 patients $(30,8 \%)$ with gastric cancer, 3 of 4 patients (75\%) patients with cancer of the pancreas, 6 of $17(35,3 \%)$ patients tumors of the colon, 6 of $10(60 \%)$ patients with cancer of the rectum and 10 of $19(52,6 \%)$ patients with liver metastases of different origin. Only 9 of 72 patients $(12,5 \%)$ with benign diseases showed urinary TGF- $\alpha$ concentrations of $>2,5 \mu / \mathrm{g}$ creatinine as cut-off level for normal persons. The TGF- $\alpha$ level elevations were found in 4 of 25 patients (16\%) with breast cancer and in 3 of 8 patients (37,5\%) with lung cancer.In contrast, the urinary EGF concentrations varied widely without significant differences between normal persons and patients with benign or malignant diseases.

Conclusions: Our data confirm that TGF- $\alpha$ may play a role in the carcinogenesis of gastrointestinal cancers such as by autocrine stimulation and may have diagnostic implications.

P2_4

\section{METHYLATION STATUS OF THE p16 TUMOR-SUPPRESSOR, DAP-kinase AND APC TUMOR- SUPPRESSOR GENES IN COLON BIOPSIES}

Teodóra Hevér-Pálfy, Orsolya Galamb, Kinga Tóth, Sándor Spisák, Barnabás Galamb, Béla Molnár, Zsolt Tulassay

Semmelweis University 2nd Department of Internal Medicine, 1088. Budapest, Hungary

Aims: The methylation patterns in tumor tissues are altered from the normal cases in numerous genes. Some of them like p16, DAP-kinase and $A P C$ genes may show a hypermethylation pattern in the $\mathrm{CpG}$ islands of their promoter regions in colorectal cancer.

The aim of the study was to examine the methylation status of these genes in colon samples.

Methods: DNA was extracted from frozen colon biopsies of 5 healthy, of 5 ulcerative colitis, of 5 tubular adenoma, of 10 CRC (colorectal cancer) patients (5 Dukes B and 5 Dukes D), using High Pure PCR Template Purification Kit. Bisulfite conversion of the samples was perfomed with CpGenome DNA Modification Kit. Methylation-specific PCR was carried out using CpGWIZ p16 Amplification Kit, CpGWIZ DAP-kinase Amplification Kit and CpGWIZ APC Amplification Kit to check the conversion and to visualize the results. Each kit contains three primers and three control DNA for unmethylated, methylated and wild-type allele sequences.

Results: All samples showed unmethylated homozygous state for methylation at the $p 16$ locus. One sample $(1 / 5,20 \%)$ was heterozigously methylated for DAP-kinase promoter region in ulcerative colitis, in CRC Dukes B and in CRC Dukes D groups. Three tubular adenoma samples $(3 / 5,60 \%)$ were in a heterozigously state for this gene. One sample $(1 / 5,20 \%)$ showed heterozigosity for methylation in group of CRC Dukes D, three CRC Dukes D samples $(3 / 5,60 \%)$ and two normal samples $(2 / 5,40 \%)$ were also heterozigously methylated at the $A P C$ locus.

Conclusions: Our results show that methylation status of $p 16$ gene does not play a meaningful role in the progress of colorectal cancer in these cases but in contrast with this, the alterations of methylation status of DAP-kinase and APC genes can contribute to the beginning of this tumorigenesis process. 


\title{
CLEAVABILITY OF HPMA-BASED CONJUGATES BEARING DOXORUBICIN BOUND BY AMIDIC BOND IS NOT A PREREQUISITE OF THEIR EFFICACY
}

\author{
O. Hovorka ${ }^{1}$, J. Strohalm² ${ }^{2}$ T. Etrych ${ }^{2}$, K. Ulbrich², B. Ŕíhová ${ }^{1}$ \\ ${ }^{1}$ Institute of Microbiology ASCR v.v.i, Prague 4, 142 20, Czech Republic, \\ ${ }^{2}$ Institute of Macromolecular Chemistry v.v.i, ASCR, Prague 6,162 06, Czech Republic
}

\begin{abstract}
Aims: Polymeric conjugates based on $N$-(2-hydroxypropyl)methacrylamide (HPMA) have been tested as potential carriers for the anticancer drug doxorubicin (Dox). The activity of proteolytically cleavable conjugates reflects the high ratio of their internalization and disintegration of the cell membrane system. In view of the latest results, cleavability of the drug from the oligopeptidic side-chains is not a necessary prerequisite for their pharmacological activity.

Materials and Methods: Intrinsic fluorescence of doxorubicin allows us to directly track and quantify the intracellular pathway of tested conjugates by fluorescence microscopy, Laser Scanning Cytometry and flow cytometry. Co-localization of conjugates with cellular organelles was analyzed by native fluorescence labeling of organelles under study. IC50 values were determined by the proliferation method based on ${ }^{3} \mathrm{H}$-thymidine incorporation.

Results: After being added to the cell culture, HPMA conjugates with doxorubicin bound through amidic bond enter the cells in seconds without the need of any active mechanism. The conjugates penetrate through the plasma membrane and are detectable in all associated cellular membranes. In contrast, hydrolytically cleavable conjugates release the drug inside endosomes/lysozomes and kill the cells by nuclear and mitochondrial damage.

Conclusions: Composition of the spacer and the manner of doxorubicin binding strongly influence intracellular distribution of polymeric drugs and thereby the mechanism of cell death.
\end{abstract}

P2_6

\section{SOMATIC HYPERMUTATION IN THE REARRANGED VH BUT NOT IN THE V-LAMBDA GENES IN EBV TRANSFORMED RHEUMATOID FACTOR-PRODUCING LYMPHOBLASTOID CELL LINE}

Reuven Laskov, Ilan Chezar, Liat Lobl-Lavi, Yaniv Gil, and Michael Steinitz

Hebrew University-Hadassah Medical School, Jerusalem, Israel

Aims: To find out whether Epstein-Bar virus (EBV) transformed lymphoblastoid cell lines (LCLs) exhibit somatic mutations in their Ig variable genes during in vitro growth.

Methods: The sequences of the rearranged $\mathrm{V}_{\mathrm{H}} / \mathrm{V}$-Lambda of a monoclonal LCL which secretes an IgM rheumatoid factor (RF-line) and of the $\mathrm{V}_{k}$ genes of cord blood (CB)-derived LCLs were determined.

Results: EBV infection of adult and cord blood peripheral lymphocytes results in a rapid induction of the expression of activation-induced cytidine deaminase (AID), a mutator responsible for somatic hypermutation (SHM) in IgV genes. Whereas the rearranged $\mathrm{V}_{\kappa}$ genes of CB-LCLs were devoid of SHM, the $\mathrm{V}_{\mathrm{H}}$ gene of the RF-line exhibited an ongoing process of SHM at a frequency of $1.5 \times 10^{-3} \mathrm{mut} / \mathrm{bp}$. The mutations were preferentially targeted to the WRCH/DGYW hot spot motifs and their majority $(15 / 16)$ were $\mathrm{C} / \mathrm{G}$ transitions, indicating that they were AID-mediated. Interestingly, no mutations were found in the rearranged V-lambda gene of the same cellular clones (i.e., $<1.2 \times 10^{-4} \mathrm{mut} / \mathrm{bp}$ ), indicating that specific trans-acting factors direct SHM to $\mathrm{V}_{\mathrm{H}}$ vs. V-lambda.

A relatively high proportion of the mutations affected the specific binding of the RF to its IgG antigen. Out of the 16 mutations found in the RF-line, four abrogated the binding activity of the antibody to IgG and two mutations affected the affinity of the binding. One mutation generated a stop codon. Overall, 11/16 of the cellular clones no longer produced an active RF antibody or totally lost the ability to produce an immunoglobulin.

\section{Conclusions:}

1) Induction of AID and SHM in the $V_{H}$ of adult-LCLs may explain the occasional loss of antigen binding-activity which takes place in antibody secreting LCLs.

2) The associations of EBV infection and B-cell malignancies may be mediated by induction of AID and its mutatory activity. 


\section{THE POSITIVE TRANSCRIPTION ELONGATION FACTOR B MEDIATES MYOCYTE ENHANCER FACTOR 2-DEPENDENT TRANSCRIPTION}

Masanori Nojima, Hiromu Suzuki, Minoru Toyota, Takashi Tokino, Mitsuru Mori, Koh Fujinaga, Kohzoh Imai, Yasuhisa Shinomura

Dept. Of Public Health, Sapporo Medical University School of Medicine, S-1, W-17, Chuo-ku, Sapporo, Hokkaido, 060-8556 Japan

Aims: Recently, increasing numbers of studies have uncovered mechanisms of cellular transcription regulated by $\mathrm{P}-\mathrm{TEFb}$. P-TEFb is an essential regulator for the complex transcriptional program in muscle cell function and development. In the present study, we demonstrate that P-TEFb activates transcription mediated by the myocyte enhancer factor 2 (MEF2)-family transcription factors in myocyte-derived cells.

Method: We investigated whether there is a functional relationship between P-TEFb and MEF2 using a reporter gene system with/without knocking down the expression of endogenous CycT1 by small interferance (si) RNA. Co-immunoprecipitation analyses were performed to determine whether P-TEFb interacts with MEF2. Coimmunoprecipitation analyses. The mRNA levels of MEF2-target genes were measured by RT-PCR. Finally, chromatin immunoprecipitation (ChIP) analyses to examine whether P-TEFb associates with the promoters of MEF2-target genes.

Results: Knockdown of the endogenous CycT1 subunit of P-TEFb by siRNA abolished MEF2-reporter gene activity and endogenous MEF2-target gene expression. Over-expression of P-TEFb subunits enhanced MEF2-dependent reporter gene expression. CycT1 interacted with MEF2 through its N-terminal cyclin box region. Furthermore, the enhancement of MEF2 activity by serum starvation was abolished in the presence of siRNA against CycT1. Finally, CycT1 was detected at the MEF2-binding regions in the promoters of the endogenous MEF2-target genes MCK, GLUT4, Nur 77, and c-Jun. These results indicate that $\mathrm{P}-\mathrm{TEFb}$ mediates MEF2-dependent transcription.

Conclusions: These results indicate that P-TEFb mediates MEF2-dependent transcription, thereby providing a fundamentally important regulatory mechanism underlying the transcriptional program in muscle cells. Its further study may also suggest a new way to elucidate the mechanism of carcinogenesis.

P2_8

\section{ALTERATIONS OF DIHYDROPYRIMIDINE DEHYDROGENASE (DPD) AND PYRIMIDINASE (DPYS) ARE NOT PREDICIVE MARKERS OF TOXICITY IN ONCOLOGY PATIENTS TREATED BY FLUOROPYRIMIDINES}

$\underline{\text { Julie Fidlerová }}^{1,2}$, Matěj Bílek ${ }^{3}$, Petra Kleiblová ${ }^{1,4}, J^{1}$ an Novotný2, Zdeněk Kleib1 ${ }^{1}$

${ }^{1}$ Institute of Biochemistry and Experimental Oncology, First Faculty of Medicine, Charles University in Prague, Czech Republic.jfidl@lf1.cuni.cz

${ }^{2}$ Department of Oncology, First Faculty of Medicine, Charles University in Prague, Czech Republic.

${ }^{3}$ Institute of Forensic Medicine and Toxicology, First Faculty of Medicine, Charles University in Prague, Czech Republic.

${ }^{4}$ Department of Obstetrics and Gynecology, First Faculty of Medicine, Charles University in Prague, Czech Republic.

Aims:Inborn metabolic deficiency of rate-limiting enzyme of the pyrimidine catabolic pathway - DPD (EC 1.3.1.2) - is supposed to be the causative factor of toxicity following treatment with fluoropyrimidines, the frequently used chemotherapeutics. Over 40 genetic changes were characterized in patients with severe toxicity and 7 of them (M166V, M182K, V335L, I560S, A777S, D949V and IVS 14+1G >A) are considered as disease-causing alterations.

Method:We have performed mutation analysis of $D P D$ in 61 patients with severe toxicity (grade III - IV according to NCIC CTC) and in 36 patients with good toleration of 5-FU treatment (grade 0-I) based on sequencing of cDNA from DPD mRNA (in 3 overlapping PCR fragments) and/or DHPLC of PCR fragments covering particular exons of the gene. Population frequency of found DPD genetic alterations was assessed in representative cohort of 171 non-cancer controls by DHPLC.

Mutation analysis using DHPLC was performed for all 9 coding exons of DPYS gene.

Results:We have characterized eight different variants of coding sequence in analyzed samples in the group of patients with toxicity. Besides known polymorphisms [C29R, S534N, I543V, V732I, c.1896C > T (silent)], we have found M166V, deletion of exon 14 (DPYD*IVS 14+1G>A), previously undescribed sequence variant c.187A>G (K63E) of DPD and two 
other intronic polymorphisms flanking to coding sequence of exon 13 (IVS13 $+39 \mathrm{C}>\mathrm{T}$, IVS13 $+40 \mathrm{~A}>\mathrm{G}$ ). The frequency of found genetic alterations were similar in both analyzed groups of patients with/without toxicity. Moreover, these frequencies were comparable to that detected in control cohort.

Similarly, the frequently found genetic alterations of DPYS (c.-1T>C; c.15 G>A; c.216C $>$ T; IVS1-58T $>$ C and IVS2 $+20 A>G$ ) occurs with the same frequency between both groups of analyzed patients.

Conclusions:Our results show that despite the high frequencies of genetic alterations presented in DPD and DPYS - their character and frequencies in analyzed populations are comparable, and therefore do not elucidate the fluoropyrimidine toxicity during treatment of oncology patients.

Acknowledgements.

Supported by grant IGA MZ CR 1A8708-4

P2_9

\title{
QUANTITATIVE ELISA STUDY OF MMPS AND TIMPS EXPRESSION PATTERN IN THE TUMORS AND PLASMA OF COLORECTAL CANCER PATIENTS
}

\author{
Elena Gershtein, Catherine Korotkova, Alexander Scherbakov, Vasiliy Prorokov, and Nicolai Kushlinsky \\ Russian N.N.Blokhin Cancer Research Centre RAMS. Kashirskoye shosse, 24.115478 Moscow. Russia
}

Matrix metalloproteinases (MMPs) and their tissue inhibitors (TIMPs) play an important role in cancer invasion, metastasis and neoangiogenesis. Several anti-MMP-targeted drugs are already under evaluation. The aim of this study was to compare the tumor expression of various MMPs and TIMPs and the plasma levels of corresponding proteins.

The study group enclosed 20 stage III-IV colorectal cancer patients. MMPs 3, 7, 9 and 13, and TIMPs 1 and 4 levels were determined by standard ELISA kits in blood plasma obtained before and 1-3 weeks after surgery, as well as in the cytosols of cancer and adjacent histologically unchanged colon mucosa tissues.

Tumor MMP-3, MMP-7, MMP-13 and TIMP-1 levels were significantly increased as compared to corresponding adjacent mucosa values in $70-90 \%$ of the patients. MMP-9 and TIMP-4 levels in the tumors and adjacent tissues did not differ significantly, and were increased in only 60 and $45 \%$ of the tumors, respectively. Pretreatment plasma concentrations of all the analyzed proteins (except MMP-9) did not significantly exceed the accepted normal values and did not decrease after the excision of the tumor. No meaningful correlations were revealed between the levels of individual proteins in the tumor and in plasma, as well as between various MMPs and/or TIMPs in tumors or plasma.

We also failed to reveal any significant associations between either tissue or plasma MMPs and TIMPs levels and such clinicopathlogic characteristics as disease stage, tumor localization and degree of contiguous spread, lymph node involvement and presence of distant metastases.

Thus, according to our data, MMPs 3, 7 and 13, as well as TIMP-1 are overexpressed in colorectal cancer, and can be considered as potential tissue, but not circulating markers for this disease as well as potential objects for moleculartargeted anti-metastatic therapy.

Acknowledgements. This study was supported by RFBR - grant 06-03-32128. 


\author{
ANTI-EGFR TARGETED POLYMERIC CONJUGATES AND MECHANISM OF THEIR \\ INTERNALIZATION \\ J. Boucek $^{1,2}$, T.Mrkvan ${ }^{1}$, O.Hovorka ${ }^{1}$, J. Strohalm ${ }^{3}$, D.Plocova ${ }^{3}$, V. Subr ${ }^{3}$, J. Betka ${ }^{2}$, K. Ulbrich ${ }^{3}$, B. Rihova ${ }^{1}$ \\ ${ }^{1}$ Institute of Microbiology, ASCR, v.v.i., Videnska 1083, 142 20, Prague 4, Czech Republic \\ ${ }^{2}$ Department of Otorhinolaryngology, Head and Neck Surgery, The First Medical Faculty, Charles University, \\ University Hospital Motol, V Uvalu 84, 150 06, Prague 5, Czech Republic \\ ${ }^{3}$ Institute of Macromolecular Chemistry, ASCR, v.v.i., Heyrovsky sq. 2, 162 06, Prague 6, Czech Republic
}

\begin{abstract}
Aims:
It has already been published that water-soluble synthetic polymers based on $N$-(2-hydroxypropyl)methacrylamide (HPMA) were recognized as useful carriers in targeted drug delivery. Receptor-mediated endocytosis was described as the main mechanism for the internalization of the targeted polymeric conjugates.

Methods:

We tried to evaluate the importance of receptor mediated endocytosis for the internalization of the tested conjugates by fluorescent microscopy, by FACS kinetic measurement and by modified cytotoxic test. We preincubated FaDu cells (model line for HNSCC) in an excess of free monoclonal antibody and with three inhibitors of endocytosis - Chlorpromasine, Cytochelasin B, Genistein.

Results:

We showed that the conjugate targeted with anti-EGFR monoclonal antibody had the same rate of internalization as conjugate without targeted structure. We also showed that preincubation in excess of relevant free antibody did not influence the uptake of the targeted therapeutics, even though it blocked specific binding to the cell surface. Moreover preincubation with three inhibitors of endocytosis, which impact on different steps of the endocytitic process, did not change the rate of internalization.

Conclusion:

The process of internalization has not been described in detail until now. Polymeric conjugates containing cytotoxic drug (doxorubicin) and targeted with anti-EGFR monoclonal antibody are effective in vitro for the FaDu cell line, but our recent data indicate that receptor mediated endocytosis is not the main mechanism of their uptake. We have been studying the details of the process of internalization.

Acknowledgment: The research was supported by CSF 310/03/H147 and by CSF 305/05/2268.
\end{abstract}

\title{
P3_2
}

\section{INVESTIGATION OF THE HUMORAL IMMUNE RESPONSE TO THE TUMOR ASSOCIATED ANTIGEN PSA IN BREAST CANCER}

M.Fulker, A. Pine, F. Whiting, J. Robertson, C. Woolston, C.Chapman.

Tumor Immunology Group, Division of Breast Surgery, Clinical Sciences Building, City Hospital, Nottingham, NG5 IPB, UK

Background and Aims: Tumors produce a number of aberrant proteins which can be mutated, over-expressed, or subject to post-translational modifications. The presentation of these aberrant proteins to the immune system can induce an autoimmune response via the generation of specific tumor autoantibodies. There is increasing evidence that these autoantibodies can be detected in cancer patients and this detection could aid early cancer diagnosis. We have previously published evidence that the detection of such autoantibodies in sera from breast cancer patients can provide a clinically valuable tool as an aid to cancer diagnosis ${ }^{1}$.

Prostate specific antigen (PSA) is a well recognised tumor antigen routinely measured in prostate cancer. There have also been reports of its presence in the circulation of individuals with breast cancer.

The aim of this work was to investigate whether autoantibodies to PSA exist in individuals with breast cancer.

Materials and Methods: cDNA encoding PSA was amplified and cloned into bacterial expression vectors and expressed in E.Coli BL21 competentent cells. The expressed recombinant protein was purified using the AKTA prime purification system.

Sera from individuals with primary invasive and advanced breast cancer, as well as individuals who had no evidence of disease, were investigated for the presence of autoantibodies to recombinant PSA.

Summary: Autoantibodies to PSA were identified in individuals with both primary and advanced breast cancer, at levels of sensitivity and specificity that suggest this antigen may provide a useful addition to a panel assay for the identification of early breast cancer. 
TUMOR SUPPRESSION BY APOPTOSIS INDUCED WITH ANTI-ERBB-2 CHIMERIC MONOCLONAL ANTIBOBY

$\underline{\text { Yasuo Kato }}^{1}$, Hiromi Hirata ${ }^{2}$, Toshio Matsune ${ }^{3}$, Shigeru Sasaki ${ }^{4}$, Masayuki Tsujisaki $^{5}$, Yasuhisa Shinomura ${ }^{4}$, Kohzoh Imai ${ }^{4}$

${ }^{1}$ Sapporo Shirakabadai Hospital, ${ }^{2}$ Hirata Hiromi Clinic, Hakodate, ${ }^{3}$ Shirakaba Pharmacy,

${ }^{4}$ Sapporo Medical University, ${ }^{5}$ Tenshi Hospital, Sapporo, Japan

\begin{abstract}
Aims: Monoclonal antibodies (mAbs) against growth factors or their receptors have been revealed to be effective therapeutic agents for solid tumors. Trastumab (Herceptin) is a humanized mAb, which was approved by FDA for the treatment of advanced breast cancer. We developed sevral mAbs against erbB-2 and prepared a mouse-human chimeric $\mathrm{mAb}$ CH401, which showed the most potent in vitro cytolytic activity, to reduce immunogenicity and enhance effector functions.

Method: The mechanism of cytolytic activity by mAb CH401 was investigated with cultured cells, and the posibility of clinical application was studied.

Results and Conclusions: This chimeric mAb completely suppressed the growth of established human tumor xenografts in SCID mice after a single injection. Furthermore, $\mathrm{CH} 401$ characteristically showed much stronger induction of apoptosia in erbB-2-overexpressing gastric cancer cells compared to trastuzumab. Morphorogical changes and DNA fgragmentation were recognized at least $12 \mathrm{~h}$ after treatment of cultured cells with $\mathrm{CH} 401$. Although the entire picture of the molecular mechanism for induction of apoptosis with $\mathrm{CH} 401$ remains to be established, we have found that $\mathrm{CH} 401$ suppressed the cell growth by inhibiting the activation of ERK and Akt, while it induces apoptosis through the activitation of p38 mitogen-activated protein kinase (MAPK) and c-Jun N-terminal kainase (JNK).
\end{abstract}

P3_4

\title{
IMMUNOCOMPLEXES OF IL-2 AND ANTI-IL-2 MAB AS NOVEL PROMISING TOOL FOR CANCER IMMUNOTHERAPY OR COMBINED CHEMO/IMMUNOTHERAPY
}

\author{
Marek Kovár̆, ${ }^{1}$ Jakub Tomala, ${ }^{1}$ Helena Chmelová, ${ }^{1}$ Jiří Strohalm, ${ }^{2}$ Tomáš Etrych, ${ }^{2}$ Karel Ulbrich, ${ }^{2}$ and Blanka \\ Ríhová $^{1}$ \\ ${ }^{1}$ Institute of Microbiology,v.vi.., Academy of Sciences of the Czech Republic, Vídeňská 1083, 14220 Prague 4, Czech \\ Republic \\ ${ }^{2}$ Institute of Macromolecular Chemistry, Academy of Sciences of the Czech Republic, Heyrovský Square 2, 16206 \\ Prague 6, Czech Republic
}

\begin{abstract}
Aims: It has been shown that immunocomplexes of IL-2 and different anti-IL2 mAb surprisingly possess strong biological activity in vivo. Depending on which anti-IL-2 mAb is used, the different subsets of immunocompetent cells are stimulated. The aim of this study was to elucidate whether such immunocomplexes can be useful for cancer immunotherapy.

Method: Using two syngenic tumor models, namely BCL1 leukemia growing in Balb/c mice and B16F10 melanoma growing in B6 mice, we tested different treatment schedules for administering either IL-2 /anti-IL-2 mAb immunocomplexes alone or together with IL-12. Using TCR-transgenic mice OT-I, we examined the potential of IL-2/anti-IL-2 mAb immunocomplexes to expand adoptively transferred (into congenic strain of mice) CFSE-labeled purified OT-I CD8 ${ }^{+}$ after injecting immunogenic peptide SIINFEKL, either with or without adjuvants. NK cell activity in mice treated with IL-2/anti-IL-2 mAb immunocomplexes was also determined.

Results: First we have shown that IL-2 /anti-IL-2 mAb immunocomplexes are able to strongly enhanced expansion of activated naive $\mathrm{CD}^{+}$cells. Treatment with IL-2 /anti-IL-2 mAb immunocomplexes maintains the high expression of CD25 on expanding cells. Both tumor models showed different sensitivity to the treatment with IL-2 /anti-IL-2 mAb immunocomplexes alone or together with IL-12. We also performed experiments in which the mice with established tumors were first treated with polymeric conjugates based on poly(HPMA) and containing doxorubicin. This combined chemo/immunotheraphy seems to be particularly effective.

Conclusions: We have studied a novel approach to effectively use IL-2 in cancer immunotherapy. Using IL-2 /anti-IL-2 $\mathrm{mAb}$ immunocomplexes seems to be a promising way to enhance anti-tumor activity of IL-2 in vivo.
\end{abstract}




\title{
EFFECT OF ANTI-ANGIOGENIC THERAPY ON CYTOKINE PROFILE IN PATIENTS WITH METASTATIC COLORECTAL CANCER
}

\author{
Sanislo L. ${ }^{1}$, Jakubíková J. ${ }^{2}$, Cholujová D. ${ }^{2}$, Sedlák J. ${ }^{2}$, Kaušitz J. ${ }^{3}$ \\ ${ }^{1}$ Clinic of Radiotherapy, St. Elizabeth Cancer Institute, Bratislava, Slovakia. \\ ${ }^{2}$ Tumor Immunology Laboratory, Cancer Research Institute, Slovak Academy of Sciences, Bratislava, Slovakia. \\ ${ }^{3}$ Clinic of Laboratory Methods of Investigation, St. Elizabeth Cancer Institute, Bratislava, Slovakia
}

\begin{abstract}
Aim: Angiogenesis plays a critical role in several physiological and pathological processes, particularly in tumor growth, invasion, and metastasis. Preclinical studies have shown that murine anti-human monoclonal antibody against VEGF could inhibit the growth of human tumor xenografts and its humanized variant bevacizumab was evaluated in clinical trials as a treatment for various cancers. The aim of our study was to assess the significance of cytokines for monitoring anti-angiogenic chemotherapy with bevacizumab.

Material and methods: Plasma cytokine profiles from 10 healthy volunteers and 11 patients with metastatic colorectal cancer during anti-angiogenic chemotherapy IFL (irinotecan,5-fluorouracil,leucovorin) + bevacizumab were analysed using a multiplex-27 bead array cytokine assay. The use of Bio-plex human cytokine 27-plex panel (Bio-rad) enabled simultaneous measurement of representative inflammatory cytokines such as GM-CSF, G-CSF, IL-1 $\beta$, IL-1RA, IL-6, IL8 , and TNF- $\alpha$; Th1/Th2 distinguishing cytokines IFN- $\gamma$, IL-2, IL-4, IL-5, IL-9 and IL-10; nonspecific acting cytokines IFN- $\alpha$, IL-7, IL-12p70, IL-13, IL-15, and IL-17; chemokines eotaxin, IP-10, MCP-1, MIP-1 $\alpha$, MIP-1 $\beta$, and RANTES; and angiogenic cytokines VEGF, bFGF, PDGF.

Results: We observed significant statistical differencies between healthy controls and patients in IL- $1 \beta$ levels $2.2 \pm 0.38 \mathrm{pg} / \mathrm{ml}$ vs. $3.6 \pm 1.28 \mathrm{pg} / \mathrm{ml}(\mathrm{p}=0,02) ; \mathrm{IL}-67.7 \pm 1.36 \mathrm{pg} / \mathrm{ml}$ vs. $19.2 \pm 4.95 \mathrm{pg} / \mathrm{ml}(\mathrm{p}=0.001) ; \mathrm{IL}-84.9 \pm 1.64 \mathrm{pg} /$ $\mathrm{ml}$ vs. $15.2 \pm 10.35 \mathrm{pg} / \mathrm{ml}$ (p=0.029); IL-10 $1.7 \pm 0.33 \mathrm{pg} / \mathrm{ml}$ vs. $4.5 \pm 2.93 \mathrm{pg} / \mathrm{ml}(\mathrm{p}=0.044) ; \mathrm{MCP}-17.3 \pm 1.87 \mathrm{pg} / \mathrm{ml}$ vs. $12.5 \pm 4.39 \mathrm{pg} / \mathrm{ml}(\mathrm{p}=0.013)$; VEGF $62.4 \pm 11.81 \mathrm{pg} / \mathrm{ml}$ vs. $120.3 \pm 36.68 \mathrm{pg} / \mathrm{ml}(\mathrm{p}=0.002)$.

Conclusion: Our results showed there was a rising concentration of endogenous VEGF in plasma over baseline levels after intravenous administration of bevacizumab. The elevation of plasma VEGF was caused by decreasing clearance of circulating VEGF.
\end{abstract}

\section{P3_6}

\section{HPMA-BASED MACROMOLECULAR THERAPEUTICS REINFORCE ANTI-TUMOR IMMUNITY}

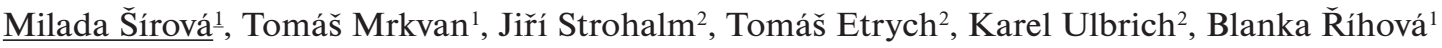 \\ ${ }^{1}$ Institute of Microbiology ASCR, v.v.i., Vídeňská 1083, 14220 Prague 4, Czech Republic \\ ${ }^{2}$ Institute of Macromolecular Chemistry ASCR, v.v.i., Heyrovský Sq. 2, 16206 Prague 6, Czech Republic
}

\begin{abstract}
Aims: Polymer-bound cytotoxic drugs were repeatedly shown to retain their anti-tumor efficacy. Moreover, they have different pharmacokinetics in comparison with the low-molecular-weight parent drugs and limited systemic toxicity. Anti-tumor efficacy of several types of $N$-[2-(hydroxypropyl)methacrylamide] (HPMA)-based conjugates of doxorubicin were evaluated in mouse tumor models in vivo, focusing on the immune responses of the host.

Method: Mouse C57BL/6 syngeneic tumors: lymphoma EL-4, and melanoma B16-F10. The tumor cells were injected subcutaneously to produce solid tumors, and conjugates were administered when the tumors were well established (therapeutic setting).

Results: HPMA-based conjugates of doxorubicin potently inhibited growth of EL-4 lymphoma and B16-F10 melanoma cells, and induced a complete cure of EL-4 lymphoma-bearing mice, depending on the dose and treatment regimen. A significant proportion of mice resisted repeated transplantation of the same tumor, when left without any further treatment. The immunity was mediated by $\mathrm{CD} 8^{+}$cells and was tumor-specific. However, we were never been able to induce a complete cure of mice bearing the B16-F10 melanoma, most probably because it is an antigenic, but non-immunogenic tumor and the B16-F10 cells do not express MHC I molecules. With the lymphoma model, we could demonstrate an antitumor response already at the time of the treatment, with an escalated response following the treatment. Higher response was found in mice with regressed tumors in comparison with mice treated with the same conjugate dose (producing $80 \%$ of complete cures), but their tumors resumed growth. The specific anti-tumor immunity seems to be a prerequisite for the complete eradication of the tumors and for the development of the specific anti-tumor memory.
\end{abstract}


Conclusions: HPMA-based doxorubicin conjugates directly target solid tumor tissue and act in concert with the immune anti-tumor responses of the host, so that the specific immune reaction increases due to the treatment, leading to generation of anti-tumor memory.

Acknowledgements:

The work was funded by the Czech Science Foundation and by the Institutional Research Concept AV0Z50200510.

\title{
P3_7
}

\section{$\alpha_{\mathrm{M}} \beta_{2}$ INTEGRIN MODULATOR INHIBITS LYMPHOMA GROWTH IN VIVO AND REDUCES SQUAMOUS CELL CARCINOMA TUMOR INVASION BY BLOCKING LEUKOCYTE INFILTRATION}

\author{
Juho Suojanen $^{1,2}$, Tuula Salo², Timo Sorsa ${ }^{1} \&$ Erkki Koivunen ${ }^{3}$ \\ ${ }^{1}$ Department of Oral and Maxillofacial Diseases, Helsinki University Central Hospital, Institute of Dentistry, \\ University of Helsinki, Finland / Biomedicum Helsinki, Institute of Dentistry, Research Laboratory, P.O.B.63 \\ Helsinki, 00014 University of Helsinki, Finland / juho.suojanen@helsinki.fi \\ ${ }^{2}$ Department of Diagnostics and Oral Medicine, Institute of Dentistry, Oulu University Hospital, 90014 University of \\ Oulu, Finland \\ ${ }^{3}$ Department of Biological and Environmental Sciences, 00014 University of Helsinki, Finland
}

Aims: We have developed a leukocyte specific $\alpha_{M} \beta_{2}$ integrin targeting small molecule (IMB-10), which is capable of inhibiting leukocyte migration and recruitment in vitro and in vivo. The purpose of this study was to investigate the effects of IMB-10 as therapy for malignancies of leukocytic and carcinoma cell origins.

Methods: Effects of IMB-10 was studied using athymic mice xenografted with U937 histiocytic lymphoma, OCI-AML-3 acute myeloid leukemia and HSC-3 tongue squamous cell carcinoma.

Results: IMB-10 therapy inhibited the growth of U937 lymphoma and OCI-AML-3 leukemia xenografts and prolonged the survival of the mice with lymphoma ( $\mathrm{p}=0.02$ ). IMB-10 also reduced the infiltration of leukocytes into HSC-3 xenografts $(p=0.05)$ and inhibited the invasion potential of carcinoma cells lacking of $\alpha_{\mathrm{M}} \beta_{2}$ integrin receptors $(\mathrm{p}=0.012)$.

Conclusions: IMB-10 has potential as a therapy for leukocytic malignancies. Since it also inhibited HSC-3 carcinoma invasion probably by inhibiting leukocyte infiltration to tumor tissue in athymic mice we suggest that host inflammation process itself may affect tumor progression also in T-cell independent manner.

Acknowledgements

We would like to thank Dr. Mikael Björklund for useful advises. This work is supported in part by the Helsinki University funds, HUCH-EVO grants, Academy of Finland, Finnish Cancer Research Foundation, and the Finnish Dental Society Apollonia. 
TREATMENT OF EXPANDING TUMORS BY TARGETED HPMA COPOLYMER-BOUND
DOXORUBICIN CONJUGATES IS FOLLOWED BY SPECIFIC ANTI-TUMOR IMMUNITY

Jakub Tomala $^{1}$, Marek Kováŕr ${ }^{1}$, Tomáš Mrkvan¹, Tomáš Etrych², Jiří Strohalm², Karel Ulbrich ${ }^{2}$ and Blanka Ríhová $^{2}$

${ }^{1}$ Department of Immunology and Gnotobiology, Institute of Microbiology, v.v.i., Academy of Sciences of the Czech Republic, Vídeňská 1083, 14220 Prague 4, Czech Republic

${ }^{2}$ Institute of Macromolecular Chemistry, v.v.i., Academy of Sciences of the Czech Republic, Heyrovský Square 2, 16206 Prague 6, Czech Republic

Aims: Targeted HPMA copolymer-bound doxorubicin conjugates based on $N$-(2-hydroxypropyl)methacrylamide (HPMA) are very potent and efficient anti-cancer therapeutics. These conjugates belong to a novel and promising category of antibody-targeted polymeric anti-cancer drugs with cytotoxic, as well as immunoprotecting and immunomobilizing activity.

Method: BCL1 B-cell leukemia growing in syngenic Balb/c mice bears surface IgM, which is a unique targeting moiety, since the idiotype of this BCL1 specific IgM is expressed only on BCL1 cells. Two different types of B1 mAb-targeted conjugate, classical and star-like, were used in our experiments. Star-like and classical conjugate differ in method of synthesis and attachment of targeting moiety resulting in increased efficiency of treatment of star-like conjugate.

Results: Both conjugates have shown to be more efficient in the treatment of established BCL1 leukemia than free doxorubicin, non-targeted and human non-specific IgG-containing conjugate. Mice that were completely cured of BCL1 leukemia by administration of antibody-targeted doxorubicin-HPMA conjugate develop various degree of systemic, BCL1-specific anti-tumor resistance which possesses characteristic of long-lasting immune memory. The degree of the resistance inversely correlates with the efficacy of the treatment with B1 mAb-targeted conjugates. We have found that $\mathrm{CD}^{+}$cells as primarily determining population responsible for emergence of anti-tumor immunity.

Conclusions: Even though the BCL1 leukemia possesses strong immune system escaping mechanisms, the B1 mAbtargeted HPMA conjugates are able to completely cure mice bearing BCL1, thus breaking the immunoescaping mechanisms and establishing long-term resistance.

\title{
P3_9
}

\section{POLYMERIC MICELLES WITH DOXORUBICIN BOUND THROUGH HYDRAZON BOND - A NEW PROMISING ANTITUMOR DRUG DELIVERY SYSTEM}

David Větvička ${ }^{1}$, Ondřej Hovorka ${ }^{1}$, Martin Hrubý ${ }^{2}$, Tomáš Mrkvan ${ }^{1}$, Karel Ulbrich ${ }^{2}$, Blanka Ř́hová ${ }^{1}$

${ }^{1}$ Institute of Microbiology ASCR v.v.i., Videňská 1083, Prague 4, Czech Republic

${ }^{2}$ Institute of Macromolecular Chemistry ASCR v.vi.., Heyrovský sq. 2, Prague 6, Czech Republic

\begin{abstract}
Aims: Amphiphilic diblock copolymer of polyallylglycidyl ether and polyethylen oxide has been designed to form micelles in aquatic solutions after binding of hydrophobic doxorubicin. The micellar formulation of doxorubicin can bring some advantages such as prolonged circulation time and decreased side toxicity. The aim of this study was to determine the perspectiveness of micellar drug delivery system with $\mathrm{pH}$ sensitive release of doxorubicin.

Method: Standard proliferation assays (conversion of MTT and incorporation of ${ }^{3} \mathrm{H}$-thymidin) was performed on several human and murine cancer cell lines. The hemolytic activity against human erytrocytes has been examined by measuring concentration of released hemoglobin. Mouse strain C57BL/6 with orthotopicaly implanted syngenic T-cell lymfoma EL4 (ATCC\# TIB-39) has been used to identify curative effect of micellear conjugate of doxorubicin. For determination of maximal tolerated dose (MTD) survival and the decrease of body weight of C57BL/6 males was recorded.

Results: Direct cytostatic effect of micelles in in vitro assays showed to be rather low if compared to free doxorubicin and some water-soluble polymeric conjugates of doxorubicin. There is no hemolytic activity of micellar copolymers with or without doxorubicin. The MTD of micellar doxorubicin conjugate is $200 \mathrm{mg}$ of doxorubicin equivalent $/ \mathrm{kg}$ after intravenous aplication. Despite the low cytotoxicity in vitro we were able to cure up to $40 \%$ of experimental animals.

Conclusions: Micellar delivery system for doxorubicin seems to be a potential cytostatic agent due to increase of MTD comparing to free drug and other nonmicellar polymeric conjugates. The $40 \%$ curative effect was achieved by a dose of $37,5 \mathrm{mg}$ of doxorubicin equivalent $/ \mathrm{kg}$, so there is a broad therapeutical window for encreasing the efficacy of the treatment.
\end{abstract}




\title{
UTILITY OF MELANOMA INHIBITORY ACTIVITY (MIA) IN MALIGNANT MELANOMA PATIENTS, COMPARISON WITH S100B
}

JM. Auge, R. Molina, J. Domingo, X. Filella, S. Puig

Oncobiology Unit, Laboratory of Biochemistry, Medical School, Hospital Clinic, Villarroel 170, Barcelona 08036, Spain

To compare the sensitivity and specificity of MIA and S-100 $\beta$ in melanoma patients, we studied prospectively both tumor markers in 158 patients with no evidence of disease (NED) and 231 patients with melanoma (39 Stage II, 57 Stage III and 135 Stage IV):

Abnormal MIA (> $12 \mathrm{ng} / \mathrm{mL}$ ) and S100 (>0.2 $\mu \mathrm{g} / \mathrm{L}$ ) were found in $4.4 \%$ (median: $7.1 \mathrm{ng} / \mathrm{mL}$ ) and 1.3\% (median: $0.08 \mu \mathrm{g} / \mathrm{L}$ ) of NED patients. Sensitivity of both tumor markers were related to tumor stage $5.1 \%$ (Median $6.6 \mathrm{ng} / \mathrm{mL}$ ) and $0 \%($ Median $0.07 \mu \mathrm{g} / \mathrm{L}$ ) in Stage II; $21 \%$ (median: $8.2 \mathrm{ng} / \mathrm{mL}$ ) and 24.5\% (median: $0.14 \mu \mathrm{g} / \mathrm{L}$ ) in Stage III and 56.3\% (Median: $13.6 \mathrm{ng} / \mathrm{mL}$ ) and 60.7\% (Median: $0.30 \mu \mathrm{g} / \mathrm{L}$ ) in Stage IV for MIA and S100 respectively. Using both tumor markers simultaneously, the sensitivity increased to $33.3 \%$ and $71.1 \%$ in Stages III and IV respectively, with similar specificity: $94.9 \%$. The concentrations of both markers were also related to site of recurrence with significantly higher concentrations in patients with multiple metastases including liver (median: $21.7 \mathrm{ng} / \mathrm{mL}$ and $2.3 \mu \mathrm{g}$ ) and the lowest in those with lung and cutaneous M1 (median: $9.8 \mathrm{ng} / \mathrm{mL}$ and $0.14 \mu \mathrm{g}$ ), for MIA and S100 respectively ( $\mathrm{p}=0.01$ ). In summary. both MIA and S100 are useful markers in patients with melanoma. and the levels are related to tumor stage and site of recurrence. Likewise, the use of MIA and S-100 increase the sensitivity obtained with S-100 alone.

\section{P4_2}

\section{EXPLORATION OF CEA EXPECTED VALUES AND CUT-OFFS IN HEALTHY AND COLORECTAL CANCER PATIENTS USING VIDAS ${ }^{\circledR}$ CEA(S) ASSAY}

\author{
JM. Riedinger ${ }^{1}$ N. Chenevoy ${ }^{1}$, M. Quibriac ${ }^{2}, \underline{\text { V. Baron-Wunderle }}{ }^{2}$ and B. Inçaurgarat ${ }^{2}$ \\ ${ }^{1}$ Laboratoire de Biologie Medicale, Centre GF. Leclerc, Dijon, France, ${ }^{2}$ bioMerieux SA, Chemin de l'Orme, Marcy \\ l'Etoile, France \\ ${ }^{1}$ Laboratoire de Biologie Medicale, Centre GF. Leclerc, Dijon, France, ${ }^{2}$ bioMerieux SA, Chemin de l'Orme, Marcy \\ l'Etoile, France
}

CEA is the most commonly used marker for the follow-up and monitoring of colorectal cancer treatment. Although routinely used, its clinical relevance according to published "generic" expected values and cut-offs in different healthy and cancerous populations have been rarely challenged. In our study, we used VIDAS ${ }^{\oplus} \mathrm{CEA}(\mathrm{S})$ (bioMérieux, France) to revisit CEA expected values in an healthy population and investigated the discrimination potential of CEA levels between healthy individuals and different colorectal cancer (CRC) populations.

Our results confirmed that CEA values obtained with $\operatorname{VIDAS}^{\oplus} \mathrm{CEA}(\mathrm{S})$ in the healthy population are significantly correlated to age and are higher in smokers compared to non-smokers. In addition, significant differences are observed between healthy, non-smoking women compared to men.

We determined in the tested populations with a ROC curve analysis a cut-off of $2.5 \mathrm{ng} / \mathrm{ml}$ to help discriminating between healthy and CRC cancer patients with primary or metastatic tumors. However, further analysis revealed that CEA alone cannot allow discrimination between healthy and primary CRC patients confirming that it cannot be used for diagnosis. In addition, we show in our study that a cut-off of $4.5 \mathrm{ng} / \mathrm{ml}$ of CEA allows a significant discrimination between primary and metastatic CRC patients demonstrating the high value of CEA in the detection and follow-up of recurrent or metastatic CRC.

These results clearly demonstrate that cut-off values for CEA and their relevance in CRC are highly dependant upon patient cancer status. Each patient should therefore be considered individually, systematically addressing his/her specific CEA baseline. This should be coupled with a thorough kinetic analysis of the evolution of CEA level to optimize CEA clinical value in patient's CRC. 


\title{
OVARY CANCER ASCITES PROTEINS - 1: CHARACTERIZATON OF CA125 MONOCLONAL ANTIBODY (OC125) BINDING PROTEINS
}

\author{
Borisenko S. ${ }^{1}$, Prokopenko P. ${ }^{1}$, Shelepova V. ${ }^{2}$, Terentiev A. ${ }^{1}$ \\ ${ }^{1}$ Russian State Medical University, 117997, Moscow, Russia \\ ${ }^{2}$ Russian Oncology Research Center, Moscow, Russia
}

\begin{abstract}
Aims: Study of alpha- and gamma-proteins (ASAP, ASGP), isolated from native ascites (NAS) from patients with ovary cancer. Methods: Reaction of precipitation (RP), electrochemiluminescence method, gel filtration (GF), polyacrylamide gel electrophoresis (PAGE). After PAGE the ASAP and ASGP were isolated from NAS. Molecular weights (MW) of the proteins were determined using GF. Rabbit antiserum (RAS) and commerce RAS containing antibodies to healthy donor serum proteins $(\mathrm{AD})$ and to heavy chains $(\mathrm{HCH})$ of $\mathrm{IgG}(2 \mathrm{mg} / \mathrm{ml})$ was prepared using GF.

Results: MW of ASAP and ASGP were determined to be $230 \mathrm{kDa}$ (ASAP230) and $470 \mathrm{kDa}$ (ASGP470). Levels of CA125 were $5146 \mathrm{U} / \mathrm{mg}$ and $7.4 \mathrm{U} / \mathrm{mg}$, respectively. RP revealed HCH-IgG (titer of 1:32), albumin (titer of 1:16), $60 \mathrm{kDa}$ polypeptide (PP60) (titer of 1:16) in ASAP230 (1.7 mg/ml) isolated after PAGE. RAS to ASGP470 contained antibodies to ASGP470, IgG, ASAP230, HCH-IgG, albumin and PP60. Sera from 50 healthy donors, serum and ascites from patient V. contain CA125 in concentrations of 34.6, 287 and $3140 \mathrm{U} / \mathrm{ml}$. respectively. Using absorbed RAS RP titers from three sources were as follows: ASAP230- 1:128, 1:512, 1:512; ASGP- 1: $128 \mathrm{n}$ all samples; HCH- 1:2048, 1: 2048, 1: 4096, respectively. ASGP470 is not identical to IgG. ASGP470, ASAP230 and PP60 were not revealed in commercial IgG (0.02 U/mg of CA125). However, the IgG preparation $(122 \mathrm{mg} / \mathrm{ml})$ is dissociated during dilution and titer of the $\mathrm{HCH}$ reaches 1: 32000

Concluson: Immunoreactivity of CA125 is probably linked with the degree of ASAP230 and ASGP470 glycosylation in blood serum.
\end{abstract}

\section{P4_4}

\section{PET IMAGING OF PROSTATE CANCER WITH A HIGHLY SPECIFIC ANTI-PSMA-ANTIBODY}

Patrick Bühler ${ }^{1}$, Philipp Wolf ${ }^{1}$, Gerald Reischl ${ }^{2}$, Bernd Pichler ${ }^{3}$, Jack Shively ${ }^{4}$, Karen Alt ${ }^{1}$, Ulrich Wetterauer ${ }^{1}$ and Ursula Elsässer-Beile ${ }^{1}$

${ }^{1}$ Department of Urology, University of Freiburg, Freiburg, Germany.

${ }^{2}$ PET-Center, Division of Radiopharmacy, University Hospital Tübingen, Tübingen, Germany.

${ }^{3}$ Laboratory for Preclinical Imaging and Imaging Technology, Clinic of Radiology, University of Tübingen,

Tübingen, Germany.

${ }^{4}$ Division of Immunology and Beckman Research Institute, Duarte, California, USA.

Aims:

The prostate-specific membrane antigen (PSMA) constitutes an excellent candidate for targeting prostate cancer by virtue of its restricted expression on prostatic epithelial cells and its upregulation on prostate cancer cells. The aim of the present study was the targeting of PSMA-expressing xenografted human prostate tumors in SCID mice to enable PET imaging of these tumors.

Method:

A monoclonal antibody $(\mathrm{mAb})$, called 3/A12 was prepared from spleen cells of mice that had been immunized with native PSMA. ${ }^{64} \mathrm{Cu}$-DOTA labeled mAb 3/A12 was administered to SCID mice bearing C4-2 human prostate carcinoma xenografts. Each animal received $30 \mu \mathrm{g}$ mAb corresponding to $380 \mu \mathrm{Ci}$ via the lateral tail vein. Mice were imaged using a small animal PET tomograph (Siemens) at 6, 24, and 48 hours after injection.

Results:

This mAb 3A/12 shows a high and specific affinity to the extracellular domain of cell-adherent PSMA. Immunoreactivity of the $\mathrm{mAb}$ was not influenced by DOTA conjugation. Blood clearance was slow with a serum half life 2 to 3 days.

High resolution PET imaging with the ${ }^{64} \mathrm{Cu}$-DOTA-labeled monoclonal antibody showed a high tumor uptake and distribution in the tumor xenografts with a negligible background in the animal. In contrast, no uptake of labeled antibody was found in the control animals bearing non-PSMA-expressing DU-145 tumors.

Conclusions:

Due to its high specificity and high tumor uptake, the mAb 3A/12 represents an excellent candidate for imaging and radioimmunotherapy. 


\title{
A PANEL OF TUMOR ANTIGENS AND AUTOANTIBODIES FOR THE DETECTION OF LUNG CANCER
}

\author{
T. Colpitts, J. Russell, J. Ramirez, E. Russell, S. Stroupe
}

Abbott Laboratories, D-09CP, AP20-3, Abbott Park, IL 60064-6015, USA

\begin{abstract}
Early intervention in lung cancer is considered crucial to the success of treatment, as reflected by the longer 5year survival rates of treated early stage disease. The desire to catch this disease at early stage has prompted large population based imaging trials for those at high risk, ie., older, heavy smokers. Unfortunately, the number of such individuals is very large and the cost of such an effort would be significant.

Tumor markers have been available for many years but their utility as single markers in early detection is limited due to lack of sensitivity and/or specificity. In combination, these markers have potential for differential diagnosis and histological subtyping. However, demonstrating their performance in early detection has yet to be accomplished.

This poster presents work that combines tumor markers with autoantibodies to provide a more sensitive test for the detection of lung cancer. The improvement in sensitivity is driven by the finding that autoantibodies are stage independent. A population of 782 individuals including 259 biopsy confirmed cancers, 280 biopsy confirmed benign lung disease, and 243 normals were studied. This population was grouped into three independent cohorts - one for the development of marker panels, and the other two for validation of those panels.

One panel combines four tumor markers with four autoantibodies. Discrimination of early stage (I \& II) lung cancer from benign lung disease (AUC 0.81) and normals (AUC 0.87) was slightly less efficient than discriminating late stage lung cancer from benign lung disease (AUC 0.89) and normals (AUC 0.93). This panel showed improved results over the panel of tumor markers alone (CA125, CEA, CYFRA 21-1 TM, and proGRP) which best discriminates this population of cancer from benign and normals with an AUC 0.80 .
\end{abstract}

\section{P4_6}

\section{COMPARISON OF CATCHER ANTIBODIES IN MANUAL TPSTM ASSAY}

Ylva D’Amico $^{1}$, Ingrid Davidsson ${ }^{1}$, Christer Bengtsson ${ }^{2}$ and Helena Goike ${ }^{1}$

${ }^{1}$ IDL Biotech AB, Bromma, Sweden

${ }^{2}$ Dept. Clin. Chem. Uppsala University Hospital, Uppsala, Sweden

\section{Aims:}

TPS ${ }^{\mathrm{TM}}$ - Tissue Polypeptide Specific antigen - is a well known tumor marker assay that specifically detects the welldefined M3 epitope on cytokeratin 18 . TPS ${ }^{\mathrm{TM}}$ is particularly useful in patient management during follow-up and monitoring of patients with breast-, gastrointestinal-, ovarian- and prostate carcinomas. Here, a comparative evaluation of TPS polyclonal vs. monoclonal versions is presented.

Method:

The TPS polyclonal catcher antibody was compared with a cytokeratin 18 specific monoclonal antibody, using sera from 100 healthy blood donors and 30 cancer patients. Separate kit lots were produced and tested to verify the specified demands on performance. Further a correlation study to TPS on Immulite 2000 was performed with148 breast cancer patients.

Results:

The assay characteristics are average data from two monoclonal TPS kit lots. The detection limit was determined to be $6 \mathrm{U} / 1$. The cut off value for a population of 100 blood donor sera calculated as the $95 \%$-ile was $84 \mathrm{U} / 1$. The within and between assay imprecision was 4,7 \% and 3,6\%, respectively. Recovery upon dilution of six different samples ranged from $92 \%$ to $118 \%$. Recovery after addition of a high concentration of antigen ranged from $99 \%$ to $107 \%$. There was no measurable interference from high concentrations of bilirubin, haemoglobin or lipids. No high dose hook effect could be detected for concentrations up to $25000 \mathrm{U} / 1$. The comparison of the polyclonal and the monoclonal versions gave a correlation coefficient of 0,970 . Comparing the monoclonal version with TPS on Immulite 2000 gave a correlation coefficient of 0,963 .

Conclusion:

The presented data shows that a monoclonal based TPS manual assay does show good technical and clinical performance, and that it correlates well with both the polyclonal and Immulite 2000 TPS versions. 


\title{
CHEMILUMINESCENT ASSAY (CIA) FOR THE RECEPTOR OF ALPHA FETOPROTEIN (RECAF) TO SEPARATE CANCER FROM NORMAL SERA
}

\author{
Barry Dowell ${ }^{1}$, Stephen Frost ${ }^{1}$, Janneta Tcherkassova ${ }^{2}$, Angela Gerber ${ }^{2}$, Rafael Moro ${ }^{2}$ and Ricardo Moro ${ }^{2}$ \\ ${ }^{1}$ Abbott Laboratories, 100 Abbott Park Road, Abbott Park, IL 60064-6013, United States \\ ${ }^{2}$ BioCurex, 215-7080 River Road, Richmond, BC V6X 1X5, Canada
}

\begin{abstract}
Aims: A radioimmunoassay for the receptor to alpha fetoprotein (RECAF) from BioCurex separates normal specimens from wide variety of cancers. Our aim was to develop a non-radioactive immunoassay for receptor to alpha fetoprotein (RECAF) for the early detection and screening of cancer.

Methods: The RECAF CIA assay is a competitive assay that utilizes a IgM monoclonal antibody (1.4G11) on the solid phase and acridinylated Human Milk Protein RECAF conjugate. We perform the RECAF CIA assay by mixing diluted human sera with acridinylated RECAF, and adding the mixture to a 1.4G11 coated microtiter plate. Following incubation, the washed plates are read in a chemiluminometer. BioCurex performed initial experiments and confirmation testing. Then, BioCurex shipped the RECAF CIA assay to Abbott Laboratories for testing.

Results: At BioCurex, studies using breast, gastric and other types of cancers $(\mathrm{n}=68)$ and normal samples $(\mathrm{n}=52)$ had an area under the curve (AUC) for the ROC curve of 0.954 with a cancer/normal ratio of 1.7. Initial experiments at Abbott Laboratories with prostate cancer $(n=8)$ and non cancer samples $(n=16)$ had AUC for ROC curve of 0.95 with a $/ \mathrm{N}$ ratio of 1.3 .

Conclusions: We developed a non-radioactive RECAF CIA assay that separates multiple types of cancer from normal sera with a $\mathrm{C} / \mathrm{N}$ ratio ranging from 1.3 to 1.7 . Our future studies will focus on increasing the cancer/normal ratio to create a manufacturable RECAF CIA assay.
\end{abstract}

\section{P4_8}

\section{EVALUATION OF PLASMA AND SERUM COLLECTION TUBES IN THE RECEPTOR FOR ALPHA FETOPROTEIN (RECAF) RADIOIMMUNOASSAY (RIA)}

Barry Dowell ${ }^{1}$, Petra Stieber ${ }^{2}$, Stephen Frost ${ }^{1}$, Janneta Tcherkassova ${ }^{3}$, Angela Gerber ${ }^{3}$, Irene $\mathrm{Ng}^{3}$, and Ricardo Moro $^{3}$

${ }^{1}$ Abbott Laboratories, 100 Abbott Park Road, Abbott Park, IL 60064-6013, United States

${ }^{2}$ Institut für Klinische Chemie, Klinikum Großhadern, Marchioninistraße 15, D-81366 München, Germany

${ }^{3}$ BioCurex, 215-7080 River Road, Richmond, BC V6X 1X5, Canada

Aims: BioCurex developed the RECAF RIA assay to separate normal specimens from a wide variety of cancers. Our aim in this study was to understand the impact of different serum and plasma specimen tube types on RECAF RIA assay performance.

Methods: In Munich, blood samples from 20 patients were collected into 11 different serum and plasma tube types and blinded. BioCurex obtained and tested the blinded samples using two competitive RECAF RIA assay formats. The RECAF RIA formats utilized I ${ }^{125}$ RECAF conjugate from breast cancer cell line with either microtiter plates coated with monoclonal antibody or rabbit polyclonal antibodies. Next, Abbott Laboratories received the RECAF RIA results and analysed the decoded data with Analyze-It software version 1.73, 2006. We determined tube types to be statistical equivalent when Wilcoxon and ANOVA analysis had $\mathrm{p}<0.05$.

Results: The RECAF values from both RECAF RIA assay formats were statistically equivalent for Sarstedt ${ }^{\mathrm{TM}}$ serum tube and Becton Dickinson(BD) $)^{\mathrm{TM}}$, serum clot tubes (plastic and glass), and serum separator tubes (plastic and glass). Sarstedt ${ }^{\mathrm{TM}}$ and Becton Dickinson ${ }^{\mathrm{TM}}$ Heparin plasma interfered with the RECAF RIA assays and caused $>3$-fold increase in RECAF values compared to serum tubes. Sarstedt ${ }^{\mathrm{TM}}$ EDTA plasma was statistically lower RECAF values than Sarstedt ${ }^{\mathrm{TM}}$ serum tube. Sarstedt ${ }^{\mathrm{TM}}$ EDTA, BD ${ }^{\mathrm{TM}}$ EDTA and citrate plasma results varied from equivalent to statistically lower values depending on RECAF RIA assay format.

Conclusions: The RECAF RIA assay is compatible with different serum collection tube types. Plasma collection tubes, especially heparin should be avoided in the RECAF RIA assay. 


\title{
SHORT- AND LONG-TERM STABILITY OF TIMP-1 IN PLASMA SAMPLES
}

\author{
Marie Dresse ${ }^{1}$, Dorothea Nagel ${ }^{1}$, Gerard Davis ${ }^{2}$, Barry Dowell ${ }^{2}$, Robert Doss ${ }^{2}$, Petra Stieber ${ }^{1}$ \\ ${ }^{1}$ Institute of Clinical Chemistry, Klinikum Grosshadern, University of Munich \\ ${ }^{2}$ Abbott Diagnostic Division, Chicago, USA \\ ${ }^{1}$ Institut für Klinische Chemie, Klinikum der Universität - Grosshadern, Marchioninistraße 15, 81377 München, \\ Germany
}

\begin{abstract}
Aim: During our investigation concerning the evaluation of TIMP-1 as an oncological biomarker we measured lower TIMP-1 levels in plasma samples from patients suffering from benign or malignant diseases than in samples of healthy individuals. As the samples of the first group had all been stored several months at $-80^{\circ} \mathrm{C}$ while most of the samples of the second group had been stored some hours or days at $4{ }^{\circ} \mathrm{C}$, we started to investigate whether temperature or time of plasma storage has an impact on TIMP-1 levels.

Methods: TIMP-1 was measured in EDTA-plasma using the ARCHITECT system (Abbott Diagnostics).

The short term stability of TIMP-1 in fresh EDTA blood samples was tested by centrifuging blood samples from 6 healthy persons after $1,2,3,4,6$ and 8 hours and then storing them at $4{ }^{\circ} \mathrm{C}$ and $-80{ }^{\circ} \mathrm{C}$ until measurement.

To investigate long-term stability, we compared the TIMP-1 levels in 197 plasma samples of healthy individuals measured after storage at $4{ }^{\circ} \mathrm{C}$ with another aliquot from the same 197 healthy individuals which had been stored at $-80{ }^{\circ} \mathrm{C}$ for several months.

Results: The differences in the TIMP-1 levels did not show a clear trend concerning time to centrifugation or storage temperature.

The median concentration of TIMP-1 in the 197 samples of healthy individuals measured after storage at $4{ }^{\circ} \mathrm{C}$ was 158 $\mathrm{ng} / \mathrm{ml}$ ( range from 98 to $331 \mathrm{ng} / \mathrm{ml}$ ). The median measured in the corresponding 197 samples after freezing at $-80^{\circ} \mathrm{C}$ was $111 \mathrm{ng} / \mathrm{ml}$ (range from 67 to $178 \mathrm{ng} / \mathrm{ml}$ ).

Conclusion: While TIMP-1 seems to be a stable parameter in blood samples stored up to 8 hours without centrifugation, levels drop in plasma after some months of storage at $-80^{\circ} \mathrm{C}$. Thus reliable clinical data can only be achieved if the corresponding samples are stored for a comparable period of time. Ideally, clinical information should be obtained by testing samples in a prospective study without long term storage of the samples. For potential routine application of this test, intensive investigations concerning stability and optimum sample handling are necessary.
\end{abstract}

\section{P4_10}

\section{STNF RI IS AN INDEPENDENT PROGNOSTIC FACTOR IN BREAST CANCER PATIENTS}

\author{
Malgorzata Fuksiewicz ${ }^{1}$, Janina Kaminska ${ }^{1}$, Maria Kowalska ${ }^{1}$, Beata Kotowicz ${ }^{1}$, Maryna Rubach ${ }^{3}$, Tadeusz \\ Pienkowski $^{2}$, Alicja Rysinska ${ }^{1}$ \\ ${ }^{1}$ Department of Tumor Markers, ${ }^{2}$ Breast Cancer and Reconstructive Surgery Clinic, ${ }^{3}$ Outpatient Chemotherapy \\ Clinic of the Maria Sklodowska-Curie Cancer Center and Institute of Oncology, Warsaw, Poland
}

\begin{abstract}
Aims: The use of serum cytokine measurements to assess disease-free survival (DFS) and overall survival (OS) in patients with breast cancer.

Methods: The levels of IL-1 ra, IL-6, IL-8, IL-10, sTNF RI, sTNF RII, M-CSF, VEGF, bFGF and CA 15.3 were measured in the sera of 210 previously untreated patients with breast cancer. Cytokines and CA 15.3 were assayed with the use of commercially available kits of R\&D Systems, Minneapolis and Abbott Diagnostics, respectively. For the statistical analyses the Mann-Whitney test, the Kaplan-Meier method, and Cox's regression model were applied.

Results: During 9 year follow-up, 53 patients died, 3 of which for reasons other than cancer, 58 had recurrent disease and 16 had stable disease progression. The examined cytokines and CA15.3 in patients before treatment were significantly higher than in healthy volunteers. Serum sTNF RI concentrations were found to correlate with OS and only sTNF RI, apart from stage, was shown to be an independent prognostic factor. The assessed cytokines and CA 15.3 were found not to relate to DFS.

Conclusions: Elevated pre-treatment serum sTNF RI proved to be a new, independent negative prognostic factor in patients with breast cancer. Neither the cytokine nor CA 15.3 measurements were found useful for DFS assessment.
\end{abstract}




\section{P4_11}

\section{DIAGNOSTIC CAPACITY OF TIMP-1 AS COMPARED TO CEA IN COLORECTAL CANCER}

Eva-Maria Ganser ${ }^{1}$, Ulla Lau-Werner ${ }^{2}$, Fritz Spelsberg ${ }^{2}$, Gerard Davis ${ }^{3}$, Barry Dowell ${ }^{3}$, Robert Doss ${ }^{3}$, Dorothea Nagel $^{1}$, Petra Stieber ${ }^{1}$

${ }^{1}$ Institute of Clinical Chemistry, Klinikum Großhadern, University of Munich, Germany

${ }^{2}$ Department of Surgery, Klinikum Großhadern, University of Munich, Germany

${ }^{3}$ Abbott Diagnostic Division, Chicago, USA

Institut für Klinische Chemie, Klinikum der Universität München, Großhadern, Marchioninistraße 15, 81377 München,

Germany

Aim:

To evaluate whether TIMP-1 may be a valuable biological marker in colorectal cancer (CRC) as compared to CEA following the evaluation criteria of the European Group on Tumor Markers (EGTM).

Method:

CEA and TIMP-1 were measured using the ARCHITECT system (Abbott Diagnostics).

We retrospectively investigated 383 EDTA samples, stored at $-80^{\circ} \mathrm{C}$ from 2004 to 2006 , consisting of 197 healthy individuals, 116 benign gastrointestinal diseases, 40 colon (including sigma) cancer and 30 rectal cancer.

Results:

The value levels achieved were as follows:

\begin{tabular}{|c|c|c|c|c|c|}
\hline & & healthy individuals & $\begin{array}{c}\text { benign gastro- } \\
\text { intestinal diseases }\end{array}$ & colon ca & rectal ca \\
\hline TIMP-1(ng/ml) & range & $67-178$ & $67-574$ & $118-424$ & $83-422$ \\
\hline & median & 111 & 142 & 190 & $0.6-957$ \\
\hline CEA $(\mathrm{ng} / \mathrm{ml})$ & range & $0.5-6.8$ & $0.5-7.6$ & $0.5-303$ & 0.6 \\
\hline
\end{tabular}

At a specificity of $95 \%$ for healthy individuals CEA reached a sensitivity of $42 \%$ for colon cancer (AUC 0.80 ), TIMP-1 resulted in $85 \%$ sensitivity (AUC 0.97 ), and the combination of both resulted in $67 \%$ sensitivity (AUC 0.93 ). For rectal cancer, the corresponding results were $53 \%$ sensitivity for CEA (AUC 0.84 ), $76 \%$ for TIMP-1 (AUC 0.91 ), and $80 \%$ for the combination (AUC 0.93), respectively.

Referring to the benign control group (95\% specificity), CEA reached a sensitivity of $32 \%$ for colon cancer (AUC 0.67 ) and $46 \%$ for rectal cancer (AUC 0.75), TIMP-1 reached $17 \%$ (AUC 0.77 ) for colon and $23 \%$ for rectal cancer $(0.75$ ), and the combination lead to $35 \%$ (AUC 0.79 ) in colon and $50 \%$ (AUC 0.82 ) in rectal cancer.

Conclusion:

In colon cancer as compared to healthy individuals TIMP-1 is superior to CEA and the combination is not able to increase this diagnostic efficacy. This is different in rectal cancer where the combined determination is clearly superior. As soon as the control group consists of benign gastrointestinal diseases CEA is superior to TIMP-1, because TIMP-1 is released to a higher extent by benign gastrointestinal diseases, like liver and inflammatory diseases. 


\title{
SERUM HER2/NEU LEVELS IN BREAST CANCER PATIENTS WITH DIFFERENT LEVEL OF TUMOR HER2/NEU PROTEIN EXPRESSION
}

Nicolai Kushlinsky, Elena Gershtein, Vyacheslav Shyroky, Valeriya Ermilova, Galina Tchemeris and Victor Letyagin

Russian N.N.Blokhin Cancer Research Centre RAMS. Kashirskoye shosse, 24.115478 Moscow. Russia

The aim of the study was to assess the clinical value of serum HER2/neu measurement in primary breast cancer patients with different levels of tumor HER2/neu protein expression.

The study comprised 59 primary stage I-III breast cancer patients, and 15 patients with benign breast lesions (control group). Soluble HER2/neu levels in the sera were determined preoperatively by the «HER-2/neu ELISA» kit (Dako Cytomation). In addition 10 patients were also tested 8-38 days after surgery. Tumor HER2/neu expression was assessed immunohistochemically with HercepTest ${ }^{\circledR}$.

At the first step HER2/neu expression was evaluated in the tumors of 171 randomly selected patients. According to the results of the immunohistochemical analysis 2 breast cancer groups were formed: group 1 comprised all 30 HER2positive $(2+/ 3+)$ patients, and group $2-29$ HER2-negative $(+1 / 0)$ patients matching group 1 by age, menopausal status, tumor size, and lymph node involvement.

Serum levels in groups 1 and 2 comprised $11.98 \pm 1.16$ and $9.19 \pm 0.33 \mathrm{ng} / \mathrm{ml}$, respectively $(\mathrm{p}<0.05)$. HER2/neu level in the sera of patients with benign breast lesions $(8.18 \pm 0.30 \mathrm{ng} / \mathrm{ml})$ was lower than in HER2-positive group ( $<<0.05)$, but did not differ significantly from that of HER2-negative group. The cut-off value $(10.5 \mathrm{ng} / \mathrm{ml})$ determined as the mean $\pm 2 \mathrm{SD}$ of the control was exceeded in $21 \%$ of HER2-negative and in $43 \%$ of HER2-positive patients. No statistically significant correlation was revealed between serum HER2/neu concentrations and tumor HER2/neu expression score $(\mathrm{R}=0.15$; $\mathrm{p}=0.077$ ). Comparison with clinico-pathologic features demonstrated that serum HER2 levels were elevated in older menopausal patients, as well as in those with tumor emboli in peripheral vessels. After the excision of the primary tumor serum HER 2 significantly decreased in 9/10 patients.

Thus, serum HER 2 concentration is associated with the HER2 status of primary breast cancer, and can be considered as a possible non-invasive substitute for tumor expression evaluation.

\section{P4_13}

\section{EVALUATION OF A PANEL OF TUMOR MARKERS IN PLEURAL FLUID FOR THE DIFFERENTIAL DIAGNOSIS OF BENIGN VERSUS MALIGNANT EFFUSIONS}

\author{
Christina Hall, Thomas Brezicka, Olle Nilsson
}

Fujirebio Diagnostics AB, Majnabbeterminalen, SE414 55 Göteborg, Sweden

\begin{abstract}
AIMS: The aim of this study was to investigate a panel of tumor biomarkers in pleural fluid for differential diagnosis in patients presenting with pleural effusion of unknown origin.

METHODS: A total of 65 samples from 58 consecutive patients undergoing thoracocentesis of diverse aetiologies were collected. Tumor markers in the pleural fluid were determined using commercially available EIA or RIA immunoassay kits for the established biomarkers CA125, CA15-3, CA19-9, CA242, CEA, SCC, S100, CYFRA 21-1 and NSE, and research EIA kits for the novel suggested biomarkers HE4, proGRP and SMRP.

RESULTS: Of the 58 patients, $46 \%$ were diagnosed with lung cancer/mesothelioma, $21 \%$ with other malignant diseases and the remaining 33\% with various benign disorders. At the time of thoracocentesis the diagnosis was still unknown for $41 \%$ of the patients later diagnosed with malignant disease. At $100 \%$ specificity the sensitivity for a subsequent malignant diagnosis was 78\% for CA15-3, 65\% for CEA, 62\% for CYFRA 21-1, 42\% for CA19-9 and CA242, 28\% for SMRP, $17 \%$ for NSE, $15 \%$ for HE 4 and proGRP, $12 \%$ for $\mathrm{S} 100,5 \%$ for SCC and $0 \%$ for CA125. The area under the ROC curves were were CA15-3 0.95, CYFRA 21-1 0.85, CEA 0.82, NSE 0.81, CA242 0.79, HE4 0.75, CA19-9 and SMRP 0.73, proGRP, S100 and SCC 0.71, and CA125 0.65 respectively.

CONCLUSIONS: The best single markers in order to discriminate between a benign and malignant pleural effusion was CA15-3. Even though a good accuracy was obtained for the best single marker, a panel of tumor markers selected to represent the most accurate markers for different lung cancers and other malignancies may provide a useful complement to cytology in order to rule in a malignant disease and for the differential diagnosis of lung cancer.
\end{abstract}




\section{PROTEOMIC ANALYSES OF ESOPHAGEAL SQUAMOUS CELL CARCINOMA}

Yi-Jun $\mathrm{Qi}^{1}$, Yuan-Fang Ma ${ }^{1}$, Qing-Yu He ${ }^{2}$, Yao-Wu Du${ }^{1}$, Guang-Chao Liu ${ }^{1}$, Yan-Jie $\mathrm{Li}^{1}$, George SW Tsao ${ }^{3}$, and $\underline{\text { Jen-Fu Chiu }}{ }^{3}$

${ }^{1}$ Institute of Immunology, Key Laboratory of Cellular and Molecular Immunology, College of Medicine, Henan University, Keifeng, Henan 475000, China ${ }^{2}$ Department of Chemistry and ${ }^{3}$ Department of Anatomy, University of Hong Kong, Hong Kong SAR, China

Esophageal cancer (EC) is the fourth most common malignancy and still represents a great health concern in northern China as well as in Western countries. Clinical outcome of EC is the most dismal among many types of digestive tumor due to its asymptomatic at early stage. Early detection and diagnosis of esophageal cancer is urgently needed to improve the curative rate. In this presentation, we analyzed the proteome from ESCC tumors and nearby non-cancer epithelia as well as the proteome from an esophageal immortal cell line and a cancer cell line.

Comparative proteomics found that the expression of 4 proteins, e.g. tropomyosin isoform 4 (TPM4), prohibitin, peroxiredoxin1 (PRX1), and manganese-containing superoxide dismutase (MnSOD) were up-regulated in ESCC and tumor cell lines as compare to adjacent normal epithelia as well as immortal tumor cells; and differential expressions of ten proteins including TPM1, squamous cell carcinoma antigen 1 (SCCA1), stratifin, peroxiredoxin 2 isoform a, alpha B crystalline, annexin A2, heterogeneous nuclear ribonucleoprotein L ( $\mathrm{hnRNP}$ L), triosephosphate isomerase1 (TPI), lamin $\mathrm{A} / \mathrm{C}$, and cyclophilin A (CypA) can be observed as well. We also found that the expression of stratifin, prohibitin, and SCCA1 dropped with increasing dedifferentiation of ESCC. In poor-differentiated ESCC, protein expression of annexin A2 and stratifin were absent in $45 \%$ and $64 \%$ of cancer, respectively. The functions of these differentially expressed proteins suggest the involvement of many pathways in the ESCC malignant transformation. The possibility of these altered proteins to be potential biomarkers for high-risk esophageal cancer screening requires further evaluation. This study was partially Supported by Doctor Initiation Grant of Henan University and Hong Kong Research Grants Council Grants HKU 7218/02M (to J.F.C.)

\section{P4_15}

\section{THYMIDINE KINASE AS A ADITIONAL MARKER IN SOLID TUMORS}

$\underline{\text { Inta Jaunalksne }}^{1}$, Simona Donina ${ }^{2}$, Tatjana Romanova ${ }^{1}$, Viktorija Priedite ${ }^{1}$, Jevgenijs Nikiforenko ${ }^{1}$, Jazeps Basko $^{3}$

${ }^{1}$ Clinical immunology center,VAS Paula Stradina KUS,Riga, Latvia, i.jaunalksne@stradina.lv

${ }^{2}$ Latvian Oncology center,Riga, Latvia

${ }^{3}$ Thoracal surgery center, VAS paula Stradina KUS, Riga, Latvia

Aims:

Thymidine kinase (TK) is a cell division marker, it is used as a malignant cell proliferation indicator, especially in hematological malignancies. The main method for detection of TK is the RIA method with ${ }^{125} \mathrm{~J}$, however there is little data about solid tumors and TK using ELISA detection methods.

Method:

We examined 100 patients with different diagnoses. For detection of TK we used the ELISA, Divi Tum (Biovica, Upsala).

Results:

Our control group showed an average of 4.4U/1, the lung cancer group 7.6U/1 and the benign lung disease group 3.5U/1. The gastrointestinal cancer (GIC) patient group before therapy showed $2.8 \mathrm{U} / 1$, but 10 days after surgery this increased to $10.4 \mathrm{U} / \mathrm{l}$, while 7 patients after operation had increased TK levels.

Conclusions:

The ELISA Divi Tum test system is possible to use in every day practice. Patients with lung cancer and other solid tumors had elevated TK levels. Benign lung diseases showed an average TK level under the cut off, while GIC patients had elevated TK levels early after surgery, which may indicate an inflammatory process. TK Divi Tum could be used as aditional marker as a cell proliferation indicator.

Acknowledgements. The authors are grateful to all Biovica producers. 


\title{
ESTABLISHMENT OF MONOCLONAL ANTIBODIES AGAINST ONCOPROTEIN E7 OF HIGH-RISK HUMAN PAPILLOMAVIRUSES FOR USE IN LIQUID-BASED CYTOLOGY AND DEVELOPMENT OF ELISA ASSAYS
}

\author{
Maria Karlsson, Christian Fermér and Olle Nilsson \\ Fujirebio Diagnostics AB, Majnabbeterminalen, SE-414 55 Gothenburg, Sweden
}

\begin{abstract}
Aims: To establish monoclonal antibodies (MAb) specific for the E7 oncoprotein of high-risk types of human papillomavirus (HPV) for design of sensitive and specific ELISA assays and for direct staining of E7 in cervical cancer cells with immunocytochemistry (ICC).

Methods: Mice were immunized with full-length oncoprotein E7 of HPV type 16 and 18. B-cells from mice demonstrating high anti-HPV E7 titers were fused with myeloma cells and hybridomas producing HPV E7 antibodies were selected. The reactivity and specificity of the antibodies to native E7 was studied in Western blot and ICC. The epitopes of the antibodies were mapped by determining the reactivity to phage particles displaying overlapping parts of the E7 proteins. Based on the mapping results, antibody pairs were selected and feasibility tested in the ELISA assay format.

Results: All HPV16 E7 MAb showed reactivity to the N-terminal part of the E7 protein and were able to form sandwich pairs with previously established HPV16 E7 MAb, known to target the central part of the antigen. The HPV18 E7 antibodies were mapped to five parts of the protein, and several sandwich pairs could be identified. Several MAb specifically stain E7 in cervical cancer cell lines using ICC.

Conclusions: For both E7 variants sensitive ELISA assays have been established and are being evaluated against native antigen from cancer cell lines. Furthermore, several MAb have the potential to be used in early detection of persistent malignant transformation by ICC staining of E7 oncoprotein in cervical cells.
\end{abstract}

P4_17

\section{ALTERATION IN MUC1 EXPRESSION IN BRONCHIAL EPITHELIUM IS AN EARLY MARKER FOR RECURRENT AND METACHRONOUS BRONCHIAL CARCINOMA}

Tatiana A. Karmakova, Raisa I.Yakubovskaya, Larisa V. Telegina, Elena V. Filonenko, Victor V. Sokolov, Georgy A. Frank, Valeriy I.Chissov.

Moscow Hertsen Institute of Oncology, 125284, Moscow

MUC1 mucin is a highly polymorphic molecule both in the number of peptide tandem repeats and in the carbohydrate chain structure. The ICO25 monoclonal antibody recognizes the peptide-carbohydrate epitope of MUC1 mucin. The ICO25 epitope is not revealed in ciliated epithelium of normal bronchi but is up-regulated in the peritumoral area of bronchial carcinoma. The aim of the study was to examine the significance of the ICO25 binding observed in bronchial epithelium for early bronchial carcinoma diagnostics.

The ICO25 epitope was detected using immunocytochemistry in bronchial epithelium cells obtained during bronchoscopy from patients with primary lung cancer (bronchial squamous cell carcinoma, $\mathrm{n}=48$; peripheral carcinoma, $\mathrm{n}=30$ ), metachronous lung cancer $(n=34)$, other malignancies localized in the thorax $(n=41)$ and chronic lung diseases (CLD, $\mathrm{n}=67$ ). Positive ICO25 staining in the columnar, basal and/or metaplastic bronchial epithelial cells was detected in $100 \%$ of bronchial carcinoma cases, $13 \%$ of peripheral carcinomas cases, $42 \%$ of non-lung tumors cases and $12 \%$ of CLD cases. A follow-up study of patients was performed within six months, with nine years time interval. Development of recurrent or metachronous squamous cell cancer in lung cancer patients after anti-tumor treatment was observed in $32(41 \%)$ out of 79 cases having ICO25 expression in bronchial epithelium cells. Patients having negative staining had no recurrence of the disease. Early squamous cell carcinoma in bronchus was revealed in $3(43 \%)$ out of 7 patients with CLD. The appearance of the ICO25 epitope in bronchial epithelium surpassed the morphological detection of the tumor growth for more than 3 months. Therefore, up-regulation of the MUC1-associated ICO25 epitope in respiratory epithelium is an early stage marker of cancer progression in bronchi and can be used for early bronchial carcinoma diagnostics in patients of the risk groups. 
TUMOR MARKERS AND CYTOKINES IN PATIENTS WITH CERVICAL CANCER

Beata Kotowicz ${ }^{1}$, Janina Kaminska ${ }^{1}$, Malgorzata Fuksiewicz ${ }^{1}$, Maria Kowalska ${ }^{1}$, Joanna Jonska ${ }^{\text {, , Mariusz }}$ Bidzinski $^{2}$

${ }^{1}$ Departments of Tumor Markers and ${ }^{2}$ Gynecologic Oncology of the Maria Sklodowska-Curie Cancer Center and Institute of Oncology, Warsaw, Poland

Aims:

The purpose of this study was to evaluate the potential use of assessments of serum levels of SCC, CEA, CA 125, CYFRA 21.1 and cytokines: IL-6, IL-8, VEGF, IL-1ra, M-CSF, sTNF RI, sTNF RII to improve the diagnosis of cervical cancer.

Methods:

The study comprised 166 untreated patients with cervical cancer. Concentrations of SCC, CEA, CA 125 were determined using the Abbott instruments system, of CYFRA 21.1 by Roche kits, of cytokines by the ELISA of R\&D. For the statistical analysis, Mann-Whitney U tests, Kruskall-Wallis ANOVA and Spearman rank correlation methods were applied.

Results:

The tumor markers and cytokines referred to clinical parameters of the disease. The levels of all cytokines were significantly higher $(\mathrm{p}<0.0001)$ in patients with cervical cancer than in healthy volunteers. Most cytokine levels (without M-CSF, sTNF RII) revealed higher sensitivity than the tumor markers. The levels of the examined parameters (except IL- 8 , M-CSF) significantly increased with the clinical stage. The analysis revealed that SCC, CA 125, IL-6 correlated with histological type, with significantly higher levels of CA $125(\mathrm{p}<0.0003)$ in adenocarcinomas, SCC $(p<0.002)$ and IL-6 $(0.0023)$ in squamous cell carcinomas. The relationship between serum levels of IL- $6(\mathrm{p}<0.012 ; \mathrm{R}=0.34)$ with the histological grade of cervical cancer was found.

Conclusions:

The results suggest that increased levels of cytokines sTNF RI, IL-8, IL-1ra, VEGF and IL-6 in serum could be potential tumor markers for diagnosis of cervical cancer, especially in early stage patients. The evaluation of CA 125 improves the diagnosis of cervical adenocarcinoma.

\title{
P4_19
}

\section{PROGRP IN DIFFERENTIAL DIAGNOSTICS OF SCLC AND NSCLC PATIENTS}

\author{
Wójcik E., Kulpa J., Sas-Korczyńska B., Stasik Z.
}

Center of Oncology, Cracow Division, Poland

Introduction: Sometimes is not possible to establish a diagnosis of malignancy by microscopic examination of biopsy material and then tumor markers might be helpful. The aim of this study was to assess which of the tumor markers used in lung cancer would be more effective in differential diagnostics.

Material and methods: The study included 50 patients with advanced NSCLC, 152 patients with SCLC before treatment, including a reference group. ProGRP, NSE, CYFRA 21-1, SCC-Ag and CEA were determined for all three groups.

Results: In the NSCLC group concentrations of CYFRA 21-1, SCC-Ag and CEA were significantly higher than in SCLC, concentrations of NSE and ProGRP were significantly lower. The sensitivity of tumor markers in NSCLC and SCLC were CYFRA $21-1: 76 \%$ vs. $26.3 \%$, SCC_Ag: $46 \%$ vs. $6.6 \%$, CEA: $26 \%$ vs. $30.3 \%$, NSE: $12 \%$ vs. $67.8 \%$ and ProGRP $6 \%$ vs. $73 \%$.

Significant dependencies between CYFRA 21-1 vs. CEA ( $\mathrm{r}=0.447)$ as well as CYFRA 21-1 vs. NSE $(\mathrm{r}=0.596)$ were found in NSCL, and in the SCLC group for CYFRA 21-1 vs. CEA ( $\mathrm{r}=0.305)$ and CYFRA 21-1 vs. NSE ( $\mathrm{r}=0.355)$. Moreover, in this group, there was a relationship between ProGRP vs. NSE ( $\mathrm{r}=0.321)$, and ProGRP vs. CYFRA 21-1 ( $\mathrm{r}=0.172)$. ROC curves were drawn in NSCLC with respect to SCLC, the AUC's were for CYFRA 21-1 - 0.813; SCC-Ag - 0.741 and CEA - 0.613 whereas the area under ROC curve for ProgRP was significantly higher than for NSE when comparing SCLC in respect to NSCLC, (respectively AUCs:0.885 vs. 0.789).

Conclusion: ProGRP seems to be a marker of choice in the differential diagnostics of lung cancer. 


\section{VEGF - A PROGNOSTIC FACTOR IN SMALL CELL LUNG CANCER PATIENTS?}

Kulpa J., Wójcik E., Korzeniowski S., Tarapacz J.

Center of Oncology, Cracow Division, Poland

Introduction: Angiogenesis is essential for tumor growth and metastasis. Data concerning a possible association between VEGF levels and tumor stage as well as prognosis of SCLC, are rare and contradictory. In this study, we evaluated NSE, ProGRP and VEGF dependences for stage of disease and also compared priority of these markers for prediction of patients' survival.

Material and methods: Determinations of NSE, ProGRP and VEGFA were performed in the reference group which consisted of healthy persons and patients with small cell lung cancer before treatment (SCLC-LD N=40 and SCLC-ED $\mathrm{N}=32$ )

Results: In SCLC patients before treatment, the concentrations of NSE, ProGRP and VEGFA were significantly higher than in the reference group. While significant correlation between NSE and ProGRP was observed, there was lack of such connections between tumor markers and VEGFA. In the group of patients with extensive disease, concentrations of all studied parameters were higher than in limited disease. Elevated ProGRP concentration was 2-times more frequent than VEGFA in SCLC-LD (65\% vs. 30\%); such marked dependencies there were not present in SCLC-ED (78.1\% vs. 59.4\%). Positive predictive value for VEGFA to confirm extensive disease was 61.3\%. 2-year survival of SCLC patients was assessed: apart from stages of malignancies and NSE, significant impact on survival was also found for ProGRP and VEGFA. Particularly unfavorable prognosis was presented by patients with higher than $670 \mathrm{ng} / \mathrm{ml}$ ProGRP concentrations and VEGFA levels above $420 \mathrm{ng} / \mathrm{ml}$.

Conclusion: Apart from extensive disease, simultanously evaluated ProGRP and VEGF, may be included as independent prognostic factors in SCLC.

P4_21

\section{ANALYSIS OF FREQUENCY OF AMINO ACID SUBSTITUTIONS IN DIRECT AFP-LIKE MOTIFS OF THE PROTEINS ASSOCIATED WITH EMBRYO- AND TUMORIGENESIS}

Nurbubu Moldogazieva, Innokenty Mokhosoev, Alexander Terentiev

Russian State Medical University, 117997, Ostrovityanova street, 1, Moscow, Russia

Among the structural features of the proteins regulating embryo- and tumorigenesis is the presence of EGF-like modules, each containing about 30-40 amino acid (aa) residues. Within these EGF-like modules we revealed short (heptapeptide) motifs similar to the fragment LDSYQCT of human alpha-fetoprotein (AFP) and designated as AFP-like motifs. Such motifs in direct and inverted forms were found in all EGF-like modules studied. We suggest that the AFP-like motifs may represent the shortest functionally active sites that function as signals for cell proliferation, differentiation and survival.

Analysis of amino acid substitutions in direct AFP-like motifs of 76 proteins containing in total 469 EGF-like modules allowed us to determine their functional importance. Using the local alignment programs (BLAST, FASTA) it was revealed that the most conserved residues are cysteine $(\mathrm{C})$ in the position 6 and tyrosine $(\mathrm{Y})$ in the position 4. Here, cysteine which is essential for disulfide bonding is a strictly conserved residue (100\% of frequency). Y may be predominantly replaced by $\mathrm{F}$ (phenylalanine) and together their frequencies are equal to $74.4 \%$. This may indicate the importance of aromatic aa in this position.

Hydrophobicity of aa in the position 1 are likely to be essential because the leucine (L) may be replaced mainly by proline (P) or valine (V). In total the three aa constitute $40 \%$ of all aa in this position. In the position 2 occurency of aspartic acid (D) is about $47 \%$ and it may be replaced by asparagine $(N)$ or glycine $(G)$. In position 3 serine $(S)$ may be replaced mainly by tyrosine $(\mathrm{T})$ and in total these two aa constitute about $50 \%$ of all aa in this position. It seems that they take part in hydrogen bonding. Aa in the positions 5 and 7 are the most variable ones. 


\title{
DIFFERENT FORMS OF SQUAMOUS CELL CARCINOMA ANTIGEN ( TOTAL SCCA, TOTAL SCCA1 AND TOTAL SCCA2) IN PATIENTS WITH BENIGN AND MALIGNANT DISEASES
}

\author{
R. Molina, JM. Auge, X. Filella \\ Oncobiology Unit, Laboratory of Biochemistry, Medical School, Hospital Clinic, Villarroel 170, Barcelona 08036, \\ Spain
}

Recently an EIA has been developed to measure total SCC (Squamous cell carcinoma antigen) as well as the neutral proteins subfraction (SCCA1) and the acidic protein subfraction (SCCA2) of the original TA-4 protein. The aim of the study was to evaluate the sensitivity and specificity of these three fractions, total SCC, SCCA1 and SCCA2 in patients with different pathologies.

SCCA, SCCA1 and SCCA2 determinations were performed in 38 healthy subjects, 60 patients with benign diseases and in 251 patients with malignancies of different origin. The concentrations of SCCA, SCCA1 and SCCA 2 were significantly higher in patients with cancer than in benign diseases or healthy people $(\mathrm{p}=0.0001)$. Likewise concentrations of all three parameters were significantly higher in benign diseases than in healthy subjects (SCC 0.01, SCCA1 0.002, SCCA2 0.04). The highest concentrations of all subfractions were found in patients with dermatological disorders or renal failure. Excluding these two groups of patients, serum levels of total SCC or SCCA1 or SCCA2 were similar in healthy subjects or in patients with benign diseases. In patients with cancer total SCC and SCCA2 serum levels were significantly higher in patients with squamous tumors than in those with other histological types. No differences were found in relation to SCCA1. Similar results were found in lung cancer, where the highest differences among Squamous and other histological types were found with SCCA2. By contrast, all subfractions concentrations were significantly higher in squamous gynecological tumors than in adenocarcinomas $(\mathrm{p}=0.0001,0.0001$, and 0.004 , respectively).

\section{P4_23}

\section{EVALUATION OF A NEW TUMOR MARKER, MESOMARK IN PATIENTS WITH BENIGN AND MALIGNANT DISEASES}

\author{
R. Molina, JM Auge, G. Casals, X. Filella
}

Oncobiology Unit. Laboratory of Biochemistry and Molecular Genetics. Hospital Clinico, Medical School. Barcelona, Spain

Oncobiology Unit. Laboratory of Biochemistry and Molecular Genetics. Hospital Clinico, Medical School. Villarroel 170, Barcelona 08036, Spain

To evaluate the sensitivity and specificity of a new tumor marker, Mesomark, we studied 35 healthy subjects, 100 patients with benign diseases and in 172 patients with malignancy (42 loco regional and 130 with metastasis disease). Benign diseases include 19 patients with renal failure, 18 patients with liver cirrhosis, 44 patients with benign lung diseases (24 infectious), 10 patients with benign gynaecological diseases and 19 patients with other benign diseases. None of the healthy subjects had abnormal mesomark serum levels $(>1.5 \mathrm{nmol} / \mathrm{L}$. By contrast abnormal levels of this tumor marker were found in $37 \%$ of patients with benign diseases. Abnormally high $(>1.5 \mathrm{nmol} / \mathrm{L})$ mesomark serum levels in patients with benign diseases were mainly found in patients with renal failure $(89,5 \%)$ and in patients with liver cirrhosis $(28 \%)$. When renal failure was excluded, mesomark specificity increases significantly and only abnormal serum levels were found in $8.3 \%$ of patients with liver cirrhosis.

Abnormal mesomark serum levels were found in 4,8\% of patients with digestive tract tumors, $10 \%$ of patients with breast cancer, $21 \%$ of patients with lung cancer, $58 \%$ of patients with ovarian cancer and in $70 \%$ of patients with malignant mesothelioma. Significanlty higher levels of this tumor marker were found in mesothelioma or in ovarian cancer than in other patients with metastatic cancer, independently of the presence mesothelium involvement $(p<0.001)$.

In conclusion, mesomark is an interesting tumor marker with high specificity of malignancy, excluding renal failure and with clinical interest in ovarian cancer and in malignant mesothelioma. 


\title{
POINT-OF-CARE SERUM TEST FOR CANCER DETECTION BASED ON THE RECAF CANCER MARKER
}

\author{
Janneta Tcherkassova**, Ralph Schmid, Xiaolong Hu***, Nina Lyubimova****, Ricardo Moro* \\ *BioCurex Inc., Richmond, BC, Canada, **Pacific Biosciences Research Centre, Richmond BC, Canada, \\ **** N.N. Blokhin Cancer Research Centre, Moscow, Russia. \\ ***BioCurex Inc. 215-7080 River Rd., Richmond B.C., Canada V6X 1 X5.
}

Objective: To develop a rapid test for an ambulatory cancer detection test based on the RECAF cancer marker.

Methods and Samples: The lateral flow chromatography device used is based on a competitive RECAF immunoassay that has been previously shown to be highly sensitive and specific for cancer detection in serum samples. A thin line of an anti-RECAF monoclonal antibody at $1 \mathrm{mg} / \mathrm{ml}$ in PBS containing 3\% Sucrose was traced onto a nitrocellulose strip and dried for 1 hour at room temperature. The samples were diluted $1 / 8$ and mixed with $10 \mathrm{ug} / \mathrm{ml}$ of RECAF-Gold colloid. 50 $\mathrm{ul}$ of the resulting volume was then added to the end of the nitrocellulose strip, from where the wet front advances towards the antibody line by capillary. One minute later, the presence of a red band over the antibody line means there is no detectable RECAF in the sample (Negative) while the presence of elevated RECAF in the sample is evidenced by the lack of the red band (Positive). The samples included 64 normal controls, 51 serum samples from patients with ovarian cancer [25 samples at Stage I and II (b-c) and 26 samples at Stage III (b-c)].

Results: $80.4 \%$ of ovarian cancers (Stages I-III) were detected with $88 \%$ specificity.

Conclusions: These results show that a rapid lateral flow chromatography test based on the RECAF marker can discriminate well between cancer and normal samples. This is consistent with previous results using a RIA format. When applied to the examples of early-stage ovarian cancer, the rapid test demonstrated better performance than CA-125. Once mechanically improved, the test might prove useful for a first approximation to the diagnosis of cancer at the doctor's office.

P4_25

\section{DIAGNOSTIC AND PROGNOSTIC VALUE OF SOLUBLE MESOTHELIN RELATED PROTEINS (SMRP) IN SERA OF PATIENTS WITH MALIGNANT PLEURAL MESOTHELIOMA}

Thomas Muley $^{1}$, Christa Traschütz ${ }^{1}$, Michael Meister ${ }^{1}$, Hans Hoffmann ${ }^{1}$, Hendrik Dienemann ${ }^{1}$, Felix JF Herth ${ }^{1}$, Monika Philipp ${ }^{2}$ and Joachim Schneider ${ }^{2}$

${ }^{1}$ Thoraxklinik am Universitätsklinikum Heidelberg, 69126 Heidelberg, Germany.

${ }^{2}$ Institut und Poliklinik für Arbeits- und Sozialmedizin, D-35392 Giessen, Germany.

Aims: The aim of the study was to evaluate the diagnostic accuracy and prognostic information of soluble mesothelin related proteins (SMRP) in patients with malignant pleural mesothelioma (MPM).

Methods: The study population consisted of 100 newly diagnosed MPM patients, 29 MPM patients with tumor relapse or progression after therapy, 75 patients with benign asbestosis, and 139 lung cancer patients. SMRP serum concentrations were measured with an ELISA kit (Mesomark ${ }^{\mathrm{TM}}$ Fujirebio Diagnostics, Malvern, USA). Statistical analyses were performed with SPSS 14.0 (Chicago, Illinois, USA).

Results: SMRP concentrations were significantly higher in patients with MPM compared to asbestosis $(\mathrm{p}<0.001)$ or lung cancer $(\mathrm{p}<0.001)$. The median values (range) were $1.4(0.2-31.0) \mathrm{nM}, 0.9(0.1-3.3) \mathrm{nM}$ and $0.8(0-6.0) \mathrm{nM}$, respectively. The highest SMRP concentrations were found in the epithelial subtype of MPM. SMRP (cut-off: $1.6 \mathrm{nM}$ ) reached a sensitivity of $42 \%$ with a specificity of $95 \%$ for benign asbestosis. Youden index revealed an optimal cut-off value of 1.35 $\mathrm{nM}$ (sensitivity: 53\%; specificity: 88\%). However, SMRP were also increased in $12.9 \%$ (cut-off $1.6 \mathrm{nM}$ ) or 19.9\% (cutoff $1.35 \mathrm{nM}$ ) of lung cancer patients. ROC curves resulted in an AUC of 0.72 (95\%CI: 0.66-0.79) for the discrimination between MPM and non-MPM patients $(\mathrm{p}<0.001)$. In the 29 MPM patients with relapse/progression, SMRP levels were significantly higher (Median: $4.2(0.2-51.0) \mathrm{nM} ; \mathrm{p}<0.001)$ compared to the untreated MPM patients. In 75 MPM patients with a complete follow-up, SMRP (cut-off: $3.5 \mathrm{nM}$ ) discriminated significantly $(\mathrm{p}<0.05)$ between patients with a good ( $\mathrm{n}=59$, median survival: 18.2 months) and patients with a worse prognosis ( $\mathrm{n}=16$, MS: 8.4 months).

Conclusions: SMRP serum concentration might be an useful measure in the diagnostic characterisation of MPM and for the differentiation between MPM, benign asbestosis and lung cancer patients. In addition, our results indicate that SMRP might be useful in treatment monitoring and follow-up. 


\section{ESTABLISHMENT OF ENZYME IMMUNOASSAY FOR DETERMINATION OF HE4}

Olle Nilsson, Ulrika Dahlén. Eva Röijer

${ }^{1}$ Fujirebio Diagnostics AB, Majnabbe Terminal SE-414 55 Gothenburg, Sweden

Objective: HE4 belong to a group of protease inhibitors containing a Whey acidic protein four disulfide core domain (WFDC - domain) and is known as WFDC2. HE4 has in several studies been shown to be over expressed in tissues and serum of ovarian carcinomas indicating that HE4 would be a potential serological biomarker for the management of patients with known or suspected ovarian cancer. The present study describes the development and characterization of a new enzyme immuno assay for determination of HE4 in human serum.

Method: Based on two mouse monoclonal antibodies, 2H5 and 3D8, recognizing epitopes within the C-WFDC-domain a two site sandwich immuno assay was developed. The assay was based on the use of biotinylated 2H5 MAb for capturing the HE4 antigen and immobilizing the antigen-antibody complex in streptavidin coated microwell plates in the first incubation. In the $2^{\text {nd }}$ incubation the captured HE4-2H5 complex is detected by incubation with HRP conjugated 3D8 MAb. The technical performance of the assay was characterised with respect to analytical and functional sensitivity, precision, linearity, recovery and potential interference from endogenous and exogenous substances etc according to NCCLS procedures. The assay was standardized using purified rec HE4 antigen as primary calibrator. The correlation towards the RUO HE4 EIA used in previous clinical studies was determined in subjects covering the HE4 range $10-900 \mathrm{pM}$.

Results: The developed assay showed an analytical sensitivity of $<2 \mathrm{pM}$, functional analytical sensitivity of $<5 \mathrm{pM}$ and intermediate precision $<5 \%$. The correlation with the RUO HE4 assay was HE4 EIA= 1.0298 RUO HE4 EIA $-7 ; \mathrm{r} 2=0.9785$. Conclusions: The HE4 EIA is a sensitive and reproducible method for measuring HE4 in human sera. The assay could be of value for the further clinical evaluation of HE4 in the management of patients with ovarian cancer.

P4_27

\section{PREDICTIVE AND PROGNOSTIC ROLE OF SERUM CYFRA 21-1 IN ADVANCED NONSMALL CELL LUNG CANCER: ASSOCIATION OF MARKER CHANGES AFTER TWO CYCLES OF CHEMOTHERAPY WITH CLINICAL RESPONSE AND SURVIVAL}

$\underline{\text { Benjamin Nisman }}^{1}$, Haim Biran², Vivian Barak ${ }^{1}$, Norman Heching ${ }^{1}$, Nili Ramu ${ }^{1}$ and Tamar Peretz ${ }^{1}$

${ }^{1}$ Oncology Department, Hadassah University Hospital, Jerusalem, Israel.

${ }^{2}$ Oncology Division, Sheba Medical Center, Tel Hashomer, Israel.

Aims:

Our aim was to test the predictive and prognostic significance of changes in serum CYFRA 21-1 levels after two cycles of chemotherapy in patients with advanced non-small cell lung carcinoma (NSCLC).

Method:

This study included 45 patients with measurable disease and evaluable for CYFRA 21-1 changes. Serum CYFRA 21-1 levels were measured before the start, and after two cycles of chemotherapy at time of clinical response evaluation. Survival was calculated from the date of response evaluation with the landmark of 6 weeks.

Results:

Critical CYFRA 21-1 decline after two cycles of chemotherapy ( $\geq 35 \%$ reduction over elevated pretreatment level) was calculated by applying the Younden index and defined as a marker response. From the two measures of radiological response used in this study, non-progression (NP, defined as $\mathrm{CR}+\mathrm{PR}+\mathrm{SD}$ ) had a stronger association with marker decline $(\mathrm{P}=0.0002)$ and survival $(\mathrm{P}=0.0002)$, than objective response-OR $(\mathrm{P}=0.02$ and $\mathrm{P}=0.39$, respectively). The concordance rate between the results of marker and radiological assessments at NP outcome used was $82.2 \%$. After two cycles of chemotherapy the marker response and radiological NP status were the major determinants of prolonged survival with relative risks $0.37(\mathrm{P}=0.01)$ and $0.63(\mathrm{P}=0.01)$, respectively.

Conclusions:

Clinical NP appears to be a better measure of treatment efficacy than OR. CYFRA 21-1 response after two cycles of chemotherapy and clinical response (considered as NP versus PD) are independent predictors for subsequent survival. CYFRA 21-1 response may be a reliable surrogate marker of treatment efficacy in advanced NSCLC. 


\title{
A NEW METHOD FOR DETERMINING SEROLOGICAL FORMS OF PRO GASTRIN-RELEASING PEPTIDE
}

\author{
Anders Öhrvik, Mattias Olsson, Ulrika Dahlén, Olle Nilsson \\ ${ }^{1}$ Fujirebio Diagnostics AB, Majnabbe Terminal 41455 Gothenburg, Sweden
}

Objective: ProGRP (Pro Gastrin-Releasing Peptide) is a precursor of the gut hormone gastrin-releasing peptide (GRP). This marker of small cell lung cancer has potential since it is more organ and tumor specific than other markers of relevance for lung cancer. Reproducible and sensitive measurement has however proven to be difficult. We here describe the development of a new sensitive assay for serum proGRP fragments.

Method: Monoclonal antibodies were developed from mice and polyclonal antibodies from goats by immunizing with recombinant proGRP peptide 31-98. Affinity purified polyclonal antibodies were biotinylated and Mab E146 reactive against proGRP aa 48-53 was conjugated to horseradish peroxidise. The assay, standardized against recombinant proGRP (31-98) protein, is a one-step sandwich EIA with $50 \mu$ l sample volume performed in microtiter wells coated with streptavidin.

Results: The analytical detection limit was below $2 \mathrm{ng} / \mathrm{L}$ and the measuring range covered $2-1000 \mathrm{ng} / \mathrm{L}$. Total imprecision was $<5 \%$ at $50 \mathrm{ng} / \mathrm{L}$. Linearity on dilutions ranged from $90-109 \%$. No high dose hook was noticed up to $500000 \mathrm{ng} / \mathrm{L}$. Heterophilic antibodies did not interfere in the assay. The estimated upper $95^{\text {th }}$ percentile in healthy individuals was below $25 \mathrm{ng} / \mathrm{L}$. Preliminary results of the new assay CanAg proGRP EIA $(\mathrm{ng} / \mathrm{L})=0,91 \mathrm{x}$ proGRP ELISA (Advanced Life Sciences Inc.) -3 demonstrated a high correlation between the methods. $(\mathrm{r}=0,97)$

Conclusions: CanAg proGRP EIA is a sensitive and reproducible method for measuring proGRP in human sera. The assay could be of value for the management of lung cancer after clinical validation.

\section{P4_29}

\section{PRODUCTION OF NEW MONOCLONAL ANTIBODIES TO THE ENTIRE proGRP(1-125) PEPTIDE}

Marianne Nordlund, Tone Varaas and Elisabeth Paus

Department of Medical Biochemistry, Rikshospitalet-Radiumhopitalet Medical Centre, Montebello, N0310, Oslo, Norway

Aims: Development and characterization of new monoclonal antibodies (mAbs) to pro-Gastrin Releasing peptide (proGRP) for better immunoassay and for characterization of the peptide.

Methods: Three different fragments of recombinant proGRP, proGRP (31-98), proGRP(1-98) and proGRP(-25-125) were cloned and expressed. The immunization of mice was performed with the two first forms conjugated to thyroglobulin, $\mathrm{Tg}$, and the last fused with the Fc-part of a mouse IgG2b, prior to fusions and hybridoma selections. Antibodies were characterized by Pepscan using 15-mer biotinylated peptides from amino acid 1 to 125 .

Results: Several new mAbs of high affinity were obtained using the larger fragments of recombinant proGRP fused to $\mathrm{Tg}$ or as a fusion protein to Fc. The best antibody pair suited for an immunofluorometric assay was selected. One new antibody performed particularly well together with an existing mAb to create a sensitive and robust assay for proGRP. Further, all mAbs were mapped to different parts of the molecule, covering the whole propeptide from amino acid 1 to 125.

Conclusion: Larger fragments of proGRP with possibly better inherent conformation have resulted in new and efficient monoclonal antibodies for a sensitive and robust assay. The antibodies have been mapped to cover the entire proGRP1125 molecule. 


\title{
MONOTOTAL - NEW MARKER FOR NSCLC
}

M.Prazakova, O.Topolcan, M.Casova, J. Safranek, L. Holubec jr., S.Kormunda

Immunoanalytic laboratories, ONM-OID, Medicine Faculty in Pilsen, Charles University, E.Benese 13, Plzen, 30509, Czech Republic

Introduction: MonoTotal is a novel tumor marker belonging to a group of cytokeratins. The existing cytokeratin markers used for diagnosis of NSCLC are mainly CYFRA 21-1, TPA and TPS.

Aim of study: To evaluate the diagnostic importance of MonoTotal and to compare it with cytokeratin tumor markers used in patients with NSCLC.

Methods and patient groups:

$>$ Control group, without tumor disease (24 patients).

$>$ Patients with NSCLC of I-III stage (26 patients),

wherein 9 patients had adenocarcinoma

and 17 patients had epidermoid carcinoma.

The serum levels of the following markers were assessed: MonoTotal (MT), Tissue Polypeptide-Specific Antigen (TPS), both by IRMA method (IDL Sweden), Tissue Polypeptid Antigen (TPA) also by an IRMA method (Dia Sorin Italy) and CYFRA 21-1 by the TRACE method (Brahms Germany).

\section{Results:}

Statistically significant differences of serum levels were observed between the control group and patients with NSCLC for MonoTotal, TPA and CYFRA 21-1.

Differences in sensitivity and ROC (Receiver Operating Characteristic) curve parameters for both histological types were identified.

Conclusions: MonoTotal is a more sensitive tumor marker for NSCLC than tumor markers currently in routine use. MonoTotal is elevated mainly in the NSCLC epidermoid carcinoma.

This study was supported by the grant IGA NR/9343-3 and by the Research project VZ MSM 0021620819.

\section{P4_31}

\section{OVARY CANCER ASCITES PROTEINS - 2: IDENTIFICATION OF POLYPEPTIDE NOT BINDING MONOCLONAL ANTIBODY (OC125) TO CA 125}

\author{
Prokopenko P.', Borisenko S. ${ }^{1}$, Shelepova V. ${ }^{2}$, Terentiev A. ${ }^{1}$ \\ ${ }^{1}$ Russian State Medical University, 117997, Moscow, Russia \\ ${ }^{2}$ Russian Oncology Research Center, Moscow, Russia
}

Aims: To identify the polypeptide, isolated from ascites (AS) alpha-protein - ASAP230 kDa (containing $514.6 \mathrm{U} / \mathrm{mg}$ of CA125), solving the problem of concomitant serum albumin (SA) and heavy chains of $\operatorname{IgG}(\mathrm{HCH}-\operatorname{IgG})$.

Methods: Reaction of precipitation (RP), gel filtration (GF), polyacrylamide gel electrophoresis (PAGE) elecrochemiluminescence method. Using method of GF HCH- IgG (MW $57.8 \mathrm{kDa}$ ) was isolated from IgG (1:100) and polypeptide with MW $59.9 \mathrm{kDa}$ (PP60) was isolated from ASAP230. Rabbit antiserum (RAS) against PP60 was prepared and commercial antiserum to donors proteins (AD) (Great Britain, Russia), to IgG and to heavy chains of IgG, IgA, IgM (BioRad, USA) were used. Thermostable protein with MW about $75 \mathrm{kDa}$ (TPAS75) was prepared from ASAP230 after $100^{\circ} \mathrm{C} 10 \mathrm{~min}$.

Results: After absorption of RAS to PP60 by serum abumin (SA) and HCH-IgG a direct comparison of test-systems in RP on PP60 with SA and HCH-IgG was performed and their full non-identity was demonstrated. TPAS75 was partially identical to heavy chains of IgG. CA125 concentration in the PP60 preparation was $16 \mathrm{mg} / \mathrm{ml}$. In blood serum from 50 healthy donors (SD50), in serum from patient B and the ascites titers of PP60 were equal to 1:32, 1:2048 and 1:8192, respectively. In commercial IgG the PP60 was not revealed. Antibodies to PP60 in titer of 1:20 were revealed in all commercial ADs. In the isolated preparations of PP60, the molecules reacting with $\mathrm{HCH}-\mathrm{IgG}$ and SA (blocking the reaction with PP60) are revealed.

Conclusion: The PP60 possibly represents the N-terminal region of CA125 but it functions in the complex with albumin and heavy chains of immunoglobulin. 


\section{CLINICAL VALUE OF S100 LEVELS IN SERUM OF MALIGNANT MELANOMA PATIENTS}

Natalja.S. Sergeeva, N.V. Marshutina, M.P. Mishunina, T.N. Lasutina, D.D.

Pack Moscow Herzen Research, Oncological Institute, 2nd Botkinsky pr. 3, 125284 Moscow, Russia

Aim: To investigate the clinical value of S100 levels in serum of patients with malignant melanoma (MM).

Patients and methods: Serum from 12 blood donors and 86 patients: 19 - with pigmented nevuses, 15 - with acute inflammatory diseases (AID), 52 - with melanoma (stage I-III), was collected. S100 protein concentration was measured using CanAg S100 EIA.

Results: S100 level was below cut-off (90ng/L) in normal serum (11/12 donors), 1 patient with AID and in all cases of pigmented nevi. MM-patients with elevated S100, increased from $14.3 \%$ for stage I (S100 mean $-53.5 \mathrm{ng} / \mathrm{L}$ ) up to 66.7 $\%$ for stage III (S100 mean value - $105.2 \mathrm{ng} / \mathrm{L}$ ). S100 mean level and percent of positive cases were correlated with such predictive factors as Breslow tumor thickness $(52.7 \mathrm{ng} / \mathrm{L}$ and $17 \%$ for $\mathrm{pT} 1 \mathrm{vs} 119.8 \mathrm{ng} / \mathrm{L}$ and $63 \%$ for pT4), presence/ absence of ulcerations (105.9 ng/L and 55, 6\% vs $79.5 \mathrm{ng} / \mathrm{L}$ and $25 \%$, respectively) and depth of invasion (49.9 $\mathrm{ng} / \mathrm{L}$ and $14 \%$ for level II vs $129.7 \mathrm{ng} / \mathrm{L}$ and $57 \%$ for level V). In the subgroup with stage II melanoma, mean S100 levels and percent of positive cases were significantly higher in serum of females vs males $(137.2 \mathrm{ng} / \mathrm{L}$ and $75 \% \mathrm{vs} 57.8 \mathrm{ng} / \mathrm{L}$ and $20 \%)$. S100 protein concentration was below cut off in all patients with clinical remission $(n=15)$. Elevated S100 values were found in $72 \%$ of patients with clinically confirmed progressive disease.

Conclusion: Protein S100 provides a valuable tool for monitoring malignant melanoma patients, for estimation of treatment efficacy and early detection of tumor progression.

P4_33

\section{DES GAMMA-CARBOXY PROTHROMBIN PROMOTES PROGRESSION OF HEPATOCELLULAR CARCINOMA}

Hidenori Shiraha, Tatsuya Fujikawa, Mayumi Suzuki, Naoki Ueda, Nobuyuki Takaoka, Shin-ichi Nishina, Shigetomi Tanaka, Kohsaku Sakaguchi, Yasushi Shiratori

Department of Gastroenterology and Hepatology, Okayama University Medical School, 2-5-1 Shikata-cho, 700-8558 Okayama, Japan

Aims:

Des gamma-carboxy prothrombin (DCP) is not only a tumor marker for screening of hepatocellular carcinoma (HCC) but also a prognosis indicator, as serum DCP level reflects the biological malignant potential. However its fine molecular mechanism is not elucidated yet. In the current study, we demonstrate that DCP promotes HCC progression by autocrine/ paracrine fashion.

Method:

Human HCC cell line Hep3B, SK-Hep1, and human umbilical vein vascular endothelial cell (HUVEC) were utilized. DCP was purified from conditioned media of $\mathrm{HCC}$ cell line PLC/PRF/5 using antibody affinity chromatography. Cell proliferative activity was assessed by $\left[{ }^{3} \mathrm{H}\right]$-thymidine incorporation. Cell migrative activity was assessed by in vitro wound healing assay. The activations of receptors and their downstream signalling effectors were assessed by Western blot analysis using anti-phospho-tyrosine specific antibodies.

Results:

DCP stimulated cell proliferation of HCC cells in a dose dependent manner (1.5-fold and 2.0-fold in Hep3B and SKHep1 cells, respectivily). DCP was found to bind with Met and cause autophosphorylation of Met. DCP activated STAT3 signaling pathway through JAK1. DCP stimulated both cell proliferative and migrative activities of HUVEC by 1.5fold and 2.2-fold, respectively. DCP induced autophosphorylation of VEGF-R2, leading to activation of MAPK through PLC $\gamma-1$.

Conclusions:

DCP not only acts as an autologous mitogen for HCC cell line, but also acts as a vascular endothelial growth factor. Met-JAK1-STAT3 signaling pathway is a major signaling pathway for DCP-induced cell proliferation of HCC, while DCP stimulates cell proliferation and migration in vascular endothelial cell through VEGF-R2- PLC $\gamma$-MAPK signaling pathway. DCP may be a therapeutic target for HCC. 


\title{
RELEASE OF ONCOLOGIC BIOMARKERS IN PATIENTS WITH PROSTATE CANCER
}

Frank Strittmatter ${ }^{1}$, Petra Stieber ${ }^{2}$, Christian G. Stief ${ }^{1}$ and Raphaela Waidelich ${ }^{1}$

${ }^{1}$ Department of Urology, University Hospital of Munich-Grosshadern, Munich, Germany.

${ }^{2}$ Institute of Clinical Chemistry, University Hospital of Munich-Grosshadern, Munich, Germany.

\begin{abstract}
Aims: The aim of the present study was to evaluate the clinical relevance and availability of common tumor markers used in clinical practice to detect prostate cancer.

Methods: Frozen sera of 442 patients in total were analysed for the presence of AFP, CA 125, CA 15-3, CA 19-9, CA 72-4, CEA, CYFRA 21-1, HCGB, NSE, fPSA, TPSA, (Roche Elecsys immunoassay), cPSA (ADVIA Centaur immunoassay), AP, CRP, and LDH (Olympus OSR6103, OSR6147 and OSR6128 tests). 131 of the 442 patients had a prostate cancer as proven by histological assessment. The remaining 311 patients were shown to have a benign hyperplasia of the prostate. Results: At 95\% specifity the cut-off values for the parameters were: AFP 5.5ng/ml, CA $12537.8 \mathrm{U} / \mathrm{ml}$, CA 15-3 37.2U/ml, CA 19-9 48.9U/ml, CA 72-4 6.3U/ml, CEA 5.3ng/ml, CYFRA 21-1 2.8ng/ml, HCGB 1.0mlU/ml, NSE 18.9ng/ml, TPSA $19.2 \mathrm{ng} / \mathrm{ml}$, cPSA $11.6 \mathrm{ng} / \mathrm{ml}$, AP 197U/L, CRP $6.9 \mathrm{ng} / \mathrm{ml}$ and LDH $271.8 \mathrm{U} / \mathrm{L}$ (15 patients with BPH [5\%] have been above these values). For these cut-off values sensitivities were calculated as follows: AFP 7\%, CA 125 5\%, CA 15-3 7\%, CA 19-9 $3 \%$, CA $72-4$ 7\%, CEA 9\%, CYFRA $21-1$ 7\%, HCGB 6\%, NSE 7\%, TPSA 37\%, cPSA 44\%, AP 9\%, CRP 5\% and LDH $7 \%$. In a multivariate analysis we calculated at a specifity of $95 \%$ a sensitiviy of $51 \%$ for the combination of fPSA/TPSA with TPSA, $48 \%$ for fPSA/TPSA with cPSA, $52 \%$ for PPSA/cPSA with cPSA and $48 \%$ for fPSA/cPSA and TPSA.

Conclusion: The significance of the tumor markers assessed in this study is limited due to their low sensitivity. Except for TPSA, cPSA and the ratios PPSA/TPSA and PPSA/cPSA all other parameters offer no advantage for the detection of prostate cancer and therefore should not be adopted into clinical practice. However the combination of TPSA, cPSA and the ratios PPSA/TPSA and PPSA/cPSA results in an increased sensitivity at $95 \%$ specificity as shown by multivariat analysis and therefore might prove to be of additional diagnostic value.
\end{abstract}

P4_35

\section{DETERMINATION OF APOPTOSIS INDUCED PEPTIDE IN THE HUMAN ALPHA-FETOPROTEIN STRUCTURE}

Tagirova A.K., Terentiev A, Alexandrova I.A., Moldogazieva N.T., Salmasi J.M., Poryadin G.V., Kazimirsky A.N.

Russian State Medical University, Moscow, 117997, Russia

There is a peptide fragment responsible for stimulation of activated apoptosis in the structure of alpha-fetoprotein (AFP). According to our data an initial site of $\mathrm{AFP}_{14-20}$ peptide LDSYQCT consists of a shorter sequence that can stimulate activated apoptosis. The peptide fragment AFP analogues are also identified in PSG and cytokines. The aim of our investigation was to investigate the influence of synthetic peptides LDSYQCT, YECE, YQCE, YVCE, YACE and SYKCE on expression activated receptors of lymphocytes, CD25, CD71, HLA-DR and CD95.

All investigations were carried out on peripheral blood lymphocytes from 18 patients with atopic bronchial asthma and intact lymphocytes of 20 healthy donors. The lymphocytes were cultivated under standard conditions during 16 hours. The final concentration of peptides in culture was $10^{-7} \mathrm{M}$. The lymphocytes were washed free from peptides and subsequently immunophenotyped.

The lymphocytes of the patients with the immunopathology were characterized by a limited approach to the activated apoptosis. Despite the fact that the homologous synthetic peptide YQCE is structurally similar to LDSYQCT, it has not found to influence the expression level of the CD95 receptor. However, two other peptides YECE and YVCS have this ability, and they reliably increased the expression level of the receptor CD95. This phenomenon may be explained by the stimulation of apoptosis by the peptides. The stimulation effect of the synthetic peptides YACS and SYKCE on this process has not been revealed.

Molecular mechanisms of increasing stimulation of activation apoptosis by synthetic peptide homologous $\mathrm{AFP}_{14-20}$ fragments is probably connected with its interaction with the membrane receptors of activated cells. The structural analysis showed that fragments with tyrosine in the first position and cysteine in the third position were necessary for apoptosis activation and peptide-receptors interaction. The apoptosis induced activity was inhibited by a hydrophobic amino acid in the second or forth position. 


\title{
SHORT AFP-LIKE MOTIFS WITHIN EGF-LIKE MODULES OF THE PROTEINS REGULATING EMBRYO- AND TUMORIGENESIS AS SIGNALS FOR CELL PROLIFERATION, DIFFERENTIATION AND SURVIVAL
}

\author{
Alexander Terentiev, Nurbubu Moldogazieva, Alexander Kazimirsky and Yury Tatarinov \\ Russian State Medical University, Moscow, 117997, Russia
}

Most of the proteins regulating embryonic development and tumor growth are mosaic, multimodular and polyfunctional ones. Each individual module of these proteins can function independently through binding with a specific cell surface receptor. Among these modules the most prominent are epidermal growth factor (EGF)-like modules and their repeats which function as signals for cell proliferation, differentiation and survival. Within these EGF-like modules we revealed short motifs similar to the fragment LDSYQCT of human alpha-fetoprotein (AFP) and designated as AFP-like motifs. Such AFP-like motifs in direct and inverted forms are linked by consensus octapeptide motif CXXGY/FXGX. We found the AFP-like motifs in EGF itself and in all EGF-like modules of growth factors, cell adhesion proteins, enzymes, coagulation factors.

Previously, the human AFP-derived peptide LDSYQCT has been synthesized using solid phase chemistry and tested for biological activity. It has been shown that it is able to regulate cell proliferation and activation apoptosis. So it has been demonstrated that this peptide is one of the biological active sites of AFP. In EGF itself the direct and inverted AFP-like motifs contain amino acid residues important for binding with the receptor which belongs to receptor tyrosine kinases (RTKs). We suggest that the AFP segment LDSYQCT as well as the similar motifs within EGF-like modules of other proteins may independently bind with the receptor and represent the shortest sites functioning as signals for cell proliferation, differentiation and survival. These signals may be realized through RTK-mediated signal transduction pathway.

P4_37

\section{ADULT HUMAN HEPATOCYTES EXPRESS ALPHA-FETOPROTEIN RECEPTOR WITHOUT RELATION TO THE EXISTENCE OF HEPATITIS, LIVER CIRRHOSIS, OR HEPATOCELLULAR CARCINOMA}

$\underline{\text { So Tsuboi }}{ }^{1}$, Kazuhiro Nouso ${ }^{2}$, Koji Manabe ${ }^{1}$, Yuzo Umeda ${ }^{3}$, Tetsuya Ogino ${ }^{4}$, Takahito Yagi $^{3}$, Noriaki Tanaka ${ }^{3}$ and Kazuhisa Taketa ${ }^{5}$

${ }^{1}$ Department of Hepatology, Shigei Medical Research Hospital, 2117 Yamada, Okayama 701-0202, Japan

${ }^{2}$ Department of Gastroenterology, Hepatology, Infectious disease, ${ }^{3}$ Department of Gastroenterological Surgery,

Transplant, and Surgical Oncology and ${ }^{4}$ Department of Pathological Research, Okayama University Graduate

School of Medicine, Dentistry and Pharmaceutical Sciences, 2-5-1 Shikata, Okayama 700-8558, Japan

${ }^{5}$ Geriatric Health Service Facility, Rijin Home, 6-16-17 Miyaura, Mihara, Hiroshima 723-0051, Japan

\begin{abstract}
Aims: The receptor for alpha-fetoprotein (AFP-R) is widely expressed not only by embryonic and fetal cells, but also by various kinds of cancer cells. Previously, we showed a high level of AFP-R expression in gastric cancer cells, whereas no expression in normal gastric mucosal cells. However, there has been no report whether adult human hepatocytes express AFP-R.

Method: Rabbit polyclonal antibodies against human AFP-R were used for immunohistochemical staining. The expression of AFP-R was examined in 44 adult human liver tissues (10 normal livers, 24 hepatocellular carcinomas (HCC), and 10 metastatic liver tumors).

Results: Surprisingly, not only cancer cells but also hepatocytes in normal liver tissues (NL cells) and nonmalignant tissues adjacent to HCC (NM cells) expressed AFP-R in varying degrees of staining intensity. There was no obvious difference in staining intensity and distribution in the tissue level. At the single-cell level, HCC and NL cells showed intracellular granular or reticular staining. On the other hand, NM cells tended to show relatively homogenous staining. Conclusions: AFP/AFP-R autocrine system may play a very important role not only in proliferation of HCC, but also in that of adult hepatocytes without relation to the existence of hepatitis, liver cirrhosis or HCC.
\end{abstract}




\section{A MULTI-ANALYTE BIOMARKER PANEL AND ALGORITHM PREDICT THE RISK OF CANCER IN WOMEN WITH PELVIC MASS}

W. Jeffrey Allard, Amy K. Brown, Craig Miller, Margarett Steinhoff, Geralyn Mersalian, Paul DiSilvestro, C.O. Granai, Robert Bast, Thorsten Verch, and Richard G. Moore

Applied Research Dept., Fujirebio Diagnostics, Inc., 201 Great Valley Parkway, Malvern, PA 19355, USA

Objectives: Twenty percent of all women in the US will be diagnosed with an adnexal mass at some point in their life, and approximately $20 \%$ of these women will have ovarian cancer. Previous studies have shown that the biomarker HE4 adds sensitivity to CA 125 in this patient population at a fixed specificity. We investigated the use of a logistic regression model to predict the risk of ovarian cancer in patients presenting with an adnexal mass.

Methods: Serum samples were obtained preoperatively from women undergoing surgery for an adnexal mass. Cancer was determined by histopathology of tissue taken at surgery. Serum samples were analyzed for levels of CA125 and HE4. A logistic regression model was developed using both biomarkers to predict the probability of finding cancer at the time of surgery.

Results: Among the 257 evaluable patients were 67 invasive epithelial ovarian cancers, 9 low malignant potential tumors of the ovary, 2 non-epithelial ovarian cancers, 13 non-gynecologic malignancies with metastasis to the ovary, and 166 patients with benign diseases. The logistic model defined low, moderate, and high risk groups with probabilities of finding cancer on surgery of $6 \%, 27 \%$, and $85 \%$ respectively.

Conclusions: A logistic model can be used to develop an algorithm to combine multiple biomarkers to predict risk of cancer in patients with a pelvic mass. The estimated probability could be used to triage patients to the appropriate surgeon and institution for surgery.

\section{P4_39}

\section{HE4 - A NOVEL BIOMARKER FOR OVARIAN CANCER}

Thorsten Verch, Curtis L. Glover, Heather L. Beyer, Ly Tran, Ryan D. Geschwindt, Ingegerd Hellstrom, Karl-Erik Hellstrom, Niranjan Y. Sardesai and W. Jeffrey Allard

Applied Research Dept., Fujirebio Diagnostics, Inc., 201 Great Valley Parkway, Malvern, PA 19355, USA

Background: HE4 (WFDC2) has been reported to be a potential biomarker for ovarian cancer. We report analytical and preliminary clinical studies of a quantitative research assay to measure serum HE4.

Methods: The HE4 assay is a 2-step immunoenzymatic assay in an ELISA format with a 6-point calibration curve (0-300 pmol/L). We measured HE4 by this assay in 256 apparently healthy females (reference interval study), 456 patients with nonmalignant conditions including 413 benign pelvic diseases and 339 with ovarian cancer.

Results: We determined the limit of detection, intraassay imprecision (CV), total imprecision and interference from potential serum components. HE4 antigen was stable in serum upon freeze/thaw $\left(-70^{\circ} \mathrm{C} /\right.$ room temperature $)$ and upon storage for at least 7 days at $2-8^{\circ} \mathrm{C}$. The $95^{\text {th }}$ percentile of the reference group was $77 \mathrm{pmol} / \mathrm{L}(95 \% \mathrm{CI}: 90-97 \mathrm{pmol} / \mathrm{L}$, $\mathrm{n}=256$ ), and median HE4 concentrations were significantly higher in sera from patients with ovarian cancer ( $81.4 \mathrm{pmol} /$ L, 95\% CI: 68.4-91.7 pmol/L, $\mathrm{p}<0.001, \mathrm{n}=339$ ) compared to healthy females or patients with benign conditions. HE4 sensitivity was determined at $52 \%$ in ovarian cancer.

Conclusions: The HE4 research assay is analytically robust and may be useful for the detection or differential diagnosis of ovarian cancer. 


\section{CHROMOGRANIN A}

J. Vrzalová, O.Topolčan, M. Čásová, L.Holubec jr., S.Kormunda

Immunoanalytic laboratories, ONM-OID, Medicine Faculty in Pilsen, Charles University, E.Benese 13, Plzen, 30509, Czech Republic

Objective: Chromogranin A, a protein with wide range of extracellular and intracellular functions, occurs mainly in neuroendocrine tissues and is a potentially novel tumor marker.

Aim: To monitor the possibility of usefulness of chromogranin A as serum tumor marker in prognostic process, for determination of aggresivity and endocrine activity of tumor, and for therapy monitoring for several diagnoses.

Materials and methods: The serum levels of chromogranin A were assessed by immunoradiometric analysis by commercial kits from Schering - CIS bio International (France).

Patient groups:

$>$ Control group -100 patients without oncological disease

$>$ Oncologic group - 150 patients with oncological disease (e.g. lung cancer, carcinoid, pheochromocytoma etc.)

These patients were further subdivided into groups according to diagnosise. The levels of chromogranin A were compared with clinical status at the time of primary diagnosis and during follow up (remission or progression).

Results: There was a percentage of false positive and false negative results for chromogranin A similar to other markers. For tumors with neuroendocrine secretion, elevated levels were found in more than $60 \%$. The levels correlate with clinical status.

Conclusion and discussion: Chromogranin A enables us to optimise the diagnostic process, to make precise differential diagnoses and to assess the neuroendocrine activity of tumors mainly for carcinoids and lung cancers.

This study was supported by research project MSM 0021620819

\section{P4_41}

\section{STABILITY OF PRO-GASTRIN RELEASING PEPTIDE IN SERUM AND PLASMA}

Toru Yoshimura ${ }^{1}$, Kenju Fujita ${ }^{1}$, Satoshi Kawakami ${ }^{1}$, Maya Kitahara ${ }^{1}$, Barry Dowell ${ }^{2}$

${ }^{1}$ Abbott Japan, Matsudo, Chiba, Japan;

${ }^{2}$ Abbott Laboratories, Abbott Park, IL, U.S.A

Aims:

ProGRP (pro-gastrin releasing peptide) is a tumor marker that is used as an aid in the diagnosis and management of small cell lung cancer (SCLC). The stability of the ProGRP in serum and plasma was investigated.

Methods and Results:

Synthetic ProGRP 31-98 peptide, recombinant ProGRP 31-98, or high titer ProGRP serums from patients with SCLC were added into serum, EDTA plasma, lithium heparin plasma, sodium heparin plasma, or citrate plasma. The spiked specimens were held from 0 to 7 days at $2-8 \mathrm{C}$ or at room temperature. ProGRP concentrations were determined with three diagnostic assay kits: Seramurabo ProGRP (Fuji-rebio), Imucheck ELSIA ProGRP (Sysmex), and Architect ProGRP (in development, Abbott Laboratories). ProGRP values were consistent within the three assay kits. ProGRP activities in serum were decreased to $50-70 \%$ after $2-8 \mathrm{C}$ storage for 7 days. The changes of ProGRP activity in plasma after $2-8 \mathrm{C}$ storage for 7 days were within $+/-10 \%$ range. There were no significant differences within plasma types.

Addition of a serine protease, thrombin (activated blood coagulation factor-II (Factor-IIa)), into PBS-BSA solution caused a decrease in ProGRP activity that was similar to the decrease in serum. The addition of PMSF, a serine protease inhibitor, into serum improved the ProGRP stability similar to the stability in plasma.

Conclusions:

Plasma provided longer stability of ProGRP than serum. We speculate that thrombin in serum is one of the factors that inactivate ProGRP in serum. The use of plasma samples for ProGRP may improve clinical reliability of this marker. 
P4_42

RECOVERY OF THE WHO FIRST INTERNATIONAL STANDARD 72/225 FOR AFP BY THE ABBOTT ARCHITECT AFP ASSAY

Kathleen Kula, Zachary Pfeiffer, Jimmy Thomas, John Jaraczewski

Abbott Diagnostics Division, Abbott Park, IL U.S.A.

Aim: The calibrators for the Abbott ARCHITECT AFP assay are standardized to the WHO (World Health Organization) First International Standard 72/225 for AFP. The purpose of this study is to demonstrate that the ARCHITECT AFP assay accurately recovers the WHO AFP standard.

Method: Three WHO sample levels at targets of $20 \mathrm{ng} / \mathrm{mL}, 80 \mathrm{ng} / \mathrm{mL}$, and $175 \mathrm{ng} / \mathrm{mL}$ were prepared from the WHO AFP standard using the reported conversion factor of 0.83 International Units per nanogram of AFP. All samples were prepared gravimetrically in filtered normal human male serum, stored at -70 degrees Celsius, and not subjected to multiple freeze thaw cycles. The specimens were then tested in three runs on each of four ARCHITECT instruments, with all testing distributed between two reagent lots. Because normal human male serum typically contains several $\mathrm{ng} / \mathrm{mL}$ of AFP, the AFP concentration of the normal human male serum was subtracted from each of the WHO sample levels in order to accurately measure the actual WHO AFP concentration in each sample. The corrected concentration values were used to determine the mean percent recovery for each WHO sample level and the grand mean percent recovery across all levels. Results: The mean percent recovery vs. the WHO reference material for each level is similar for all levels tested: low sample $(20 \mathrm{ng} / \mathrm{mL})=104.04 \%$, medium sample $(80 \mathrm{ng} / \mathrm{mL})=101.02 \%$, high sample $(175 \mathrm{ng} / \mathrm{mL})=100.94 \%$. The overall grand mean percent recovery across all levels of AFP WHO standard is $102.00 \%$.

Conclusion: The ARCHITECT AFP assay reads the WHO First International Standard 72/225 for AFP with accuracy, which is important for clinical use as an aid in cancer patient management and prenatal screening.

P4_43

\section{TUMOR MARKER KINETICS (TMK) OF CEA AND CA15-3 CORRELATE WITH RESPONSE IN PATIENTS UNDERGOING CHEMOTHERAPY FOR METASTATIC BREAST CANCER}

Dorit Laessig ${ }^{1}$, Dorothea Nagel ${ }^{2}$, Volker Heinemann ${ }^{1}$, Michael Untch ${ }^{3}$, Steffen Kahlert ${ }^{4}$, Ingo Bauernfeind ${ }^{4}$, Petra Stieber ${ }^{2}$

${ }^{1}$ Medical Department III, University of Munich, Klinikum Grosshadern, Germany, ${ }^{2}$ Institute of Clinical Chemistry, University of Munich, Klinikum Grosshadern, Germany; ${ }^{3}$ Gynecological Department, Helios Klinikum Berlin-Buch, Germany; ${ }^{4}$ Gynecological Department, University of Munich, Klinikum Grosshadern, Germany

${ }^{2}$ Klinikum der Universität München - Großhadern, Marchioninistraße 15, 81377 München, Germany

Aims: The aim of this retrospective analysis was to determine the correlation between tumor marker kinetics (TMK) like CEA and/ or CA 15-3 and imaging during chemotherapy of metastatic breast cancer (MBC) patients.

Material and Methods: TMK (CEA, AxSYM, Abbott; CA 15-3, Elecsys, Roche) were evaluated in MBC patients $(\mathrm{n}=77)$ at the beginning of chemotherapy for metastatic breast cancer (pre-treatment value $=\mathrm{A})$, after 20-30 days (1st intermediate value $=\mathrm{B}$ ), after 40-60 days (2nd intermediate value $=\mathrm{C}$ ) and at the time of staging with imaging techniques (D). Response to treatment was assessed by UICC criteria. Two criteria for progressive disease and two criteria for non-progressive disease based on TMK were established. The $1^{\text {st }}$ criterion for progression required that the increase from $\mathrm{A}$ to $\mathrm{C}$ had to be greater than $25 \%$, and the increase per day from $\mathrm{A}$ to $\mathrm{C}$ had also to be greater from $\mathrm{A}$ to $\mathrm{B}$. The $2^{\text {nd }}$ criterion for progression required that $\mathrm{D}$ should be $>25 \%$ than $\mathrm{A}$ and also an increase of $\mathrm{C}$ to $\mathrm{D}$ was required. The $1^{\text {st }}$ criterion for non-progressive disease required that the decrease from $\mathrm{A}$ to $\mathrm{C}$ had to be greater than $25 \%$ and $\mathrm{C}$ had to be lower than $\mathrm{B}$. The $2^{\text {nd }}$ criterion for non-progressive disease required $\mathrm{D}<25 \%$ than $\mathrm{B}$ and $\mathrm{D}$ had to be lower than $\mathrm{C}$.

Results: Fifty-four (70\%) patients showed a correlation of TMK and imaging results. In $10(13 \%)$ patients no correlation was obtained, and in $13(17 \%)$ patients no biochemical statement was possible because of divergent TMK. Using our criteria a sensitivity of $70.2 \%$ was reached.

Conclusion: We could show a correlation between TMK and imaging results. After validation in a prospective trial using our criteria in clinical practice we could improve therapeutic monitoring and reduce radiation exposure in patients with MBC. 


\title{
IMMUNOMAGNETIC BEAD ENRICHMENT OF CIRCULATING TUMOR CELLS - RECOVERY EXPERIMENTS
}

\author{
Michaela Cabinakova $^{1}$, Petra Tesarova ${ }^{1}$, Karin Malickova ${ }^{2}$, Ivana Janatkova ${ }^{2}$ \\ ${ }^{1}$ Department of Oncology, General Faculty Hospital and $1^{\text {st }}$ Faculty of Medicine of Charles University, \\ U Nemocnice 2, 12808 Prague, Czech Republic \\ ${ }^{2}$ Institute of Clinical Biochemistry and Laboratory Diagnostics, General Faculty Hospital and $1^{\text {st }}$ Faculty of \\ Medicine of Charles University, Karlovo nam.32, 12111 Prague, Czech Republic
}

\begin{abstract}
Aim: Circulating tumor cells (CTC) can be present in the blood of carcinoma patients at extremely low frequencies (less than 10 cells $/ \mathrm{ml}$ ). Our aim is to make an assay for detection of CTC that is not limited by low concentration of cells. With this purpose we tested the recovery rate of the immunomagnetic separation of cultivated human breast adenocarcinoma cells MCF-7 from the whole blood.

Methods: MCF-7 cells were serially diluted 10-fold with RPMI and 10-fold with blood from healthy donors. The concentrations used in recovery experiments were 10000, 1000, 100 and 10 cells $/ \mathrm{ml}$. We enriched tumor cells from cell suspensions by Dynabeads ${ }^{\circledR}$ technology. Superparamagnetic beads coated with monoclonal antibody Ber-EP4 specific for membrane epithelial antigens and with CD45 pan-leukocyte antigen were added to cell suspensions and isolated by using magnet (positive and negative separation, respectively). After labeling of isolated cells by monoclonal antibodies, percentage of epithelial cells in each sample was determined using flow cytometry. Specific fluorescent-labeled monoclonal antibodies against cytokeratin molecules were used.

Results: After enrichment, cell numbers collected in various procedures were different. Significant specificity was found with negative immunomagnetic beads and with combined use of negative and positive beads.

Conclusions: Accurate isolation of circulating tumor cells in the blood is necessary for cell enumeration and further characterization. The most important consequence of CTCs enumeration is whether this information will change clinical management in patients with neoplasmas.

Acknowledgements.
\end{abstract}

This work is funded by the grant of IGA of the Ministry of Health of the Czech Republic No. 9020-3.

P5_2

\section{DETECTION OF P15 GENE PROMOTER METHYLATION IN SERUM IN GASTRIC CANCER PATIENTS}

$\underline{\text { Simona Donina }}^{1,2}$, Svetlana Kozireva ${ }^{2}$, Modra Murovska $^{2}$, Viesturs Boka ${ }^{3}$

${ }^{1}$ Riga Eastern Hospital, Latvian Oncology Center, 4 Hipokrata Str., Riga, LV-1079, Latvia

${ }^{2}$ August Kirchenstein Institute of Microbiology and Virology, Rigas Stradina University, 1 Ratsupites Str., Riga,

Latvia

${ }^{3}$ P.Stradina Clinical University, 16 Dzirciema Str., Riga, Latvia

Aim:

$\mathrm{CpG}$ island methylation is an important mechanism for the inactivation of genes involved in tumorigenesis. Gastric carcinoma is one of the tumors that exhibits a high frequency of $\mathrm{CpG}$ island hypermethylation in various genes. We tested the frequency of aberrant promoter methylation of $\mathrm{p} 15$ gene in serum of patients with gastric carcinoma.

Materials and Methods:

Pre-operative serum samples obtained from 22 gastric cancer patients with histological conformed adenocarcinoma and 22 apparently healthy blood donors were subjected to methylation-specific polymerase chain reaction assay for the p15 gene. Results:

Promoter methylation of p15 gene was detected in sera of 3 out of 22 patients with gastric carcinoma. The patients with detected promoter methylation of p15 gene had I, II and IV stage of disease. None of the sera from blood donors demonstrated promoter methylation of this gene.

Conclusions:

Although these results need to be confirmed in larger studies and the methylation status of other genes should be determined, we suggest that detection of aberrant promoter methylation of cancer-related genes in serum may be useful for diagnosis and surveillance of gastric cancer. 
TRAKING-LINE USE: EXPERIENCE IN A LABORATORY ANALYSIS WITH A WIDE CANCER ASSAY PANEL

\author{
Micca Gianmatteo, Sandri Maria Teresa, Cerri Angela, Vignati, Bellati Paola
}

P.O. S. Lazzaro - Via P. Belli 26 - 12051 ALBA (cn) Italy

The Alba Hospital Laboratory

We have performed analyses using a wide assay panel for tumor markers, hormones, Hepatitis and HIV infectiology, traditional serology, Torch, EBV, Herpes, drugs of abuse, immunosuppressant drugs, IgE total and specific. The analysers used were 2 Abbott Architect, 2 Tosoh AIA 1800 ; 2 DiaSorin Liaison, 1 Dade Behring Dimension Rxl , 1 Phadia Immunocup 250.

To overcome the problem of pre-analytical handling, it was decided to install a tracking line which makes it possible to change the pre-analytical, analytical and post-analytical process; reduces the number of test-tubes required for the sample; reduced personnel time; allows rationalisation of costs.

PSA represents the $18 \%$ of total markers assayed in the Laboratory: $(24,000 / 133,000)$.

From the evaluation of 202 routine samples on Tosoh AIA 1800, a comparison between methods (Abbott Architect, Roche Elecsys, DPC Immulite, Advia Centaur, Beckman Access 2 -Hybritech, Beckman Access 2 -WHO) was made to check the commutability, correlation and possibility of overlaying the clinical information.

The Beckman assays, based on Hybritech and WHO calibration, allowed us to assess the standardisation of the methods. Data commutability was assessed by Deming regression and correlation by Spearman's non-parametric analysis. The calculated Rs are $0.9943 ; 0.9948 ; 0.9917 ; 0.9932 ; 0.9923 ; 0.9889$.

Conclusions: the methods are not commutable but are correlated; the clinical information provided can be overlaid using the same method. The problems linked with equimolarity have been overcome by the WHO 96/670 standard and the negative bias of all methods in relation to Beckman Access2 Hybritech calibration confirms it.

\title{
P5_4
}

\section{AFP ASSAY ON OLYMPUS AU3000I IMMUNOASSAY ANALYSER}

\author{
Angela Parnham, Ron Harris, Parveen Mughal
}

Dept Clinical Chemistry, North Tyneside General Hospital, Rake Lane, North Shields, Tyne and Wear, NE29 8NH, United Kingdom

We present an assessment of the AU 3000i AFP assay from Olympus Diagnostics carried out in the routine laboratory of a UK district general hospital.

The within batch imprecision of the assay using the Olympus internal QC material (mean $8.3 \mathrm{IU} / \mathrm{ml}$ ) was CV $1.23 \%$ and on-board dilution of a high patient sample, (mean 1210IU/ml), 1/10 dil, CV 2.59\%; 1/100 dil, CV 3.04\%, 1/200 dil, CV 2.38\%. Between batch imprecision using Biorad IQC material was mean $11.24 \mathrm{IU} / \mathrm{ml}, \mathrm{CV} 3.6 \% ; 67.5 \mathrm{IU} / \mathrm{ml}, \mathrm{CV} 4.1 \%$, $145 \mathrm{IU} / \mathrm{ml} \mathrm{CV} \mathrm{5.6 \% :} \mathrm{Olympus} \mathrm{IQC} \mathrm{mean} \mathrm{8.8IU/ml,} \mathrm{CV} \mathrm{6.5 \% :} \mathrm{and} \mathrm{a} \mathrm{low} \mathrm{patient} \mathrm{pool} \mathrm{mean} 2.8 \mathrm{IU} / \mathrm{ml}, \mathrm{CV} 6.2 \%$.

The patient comparison was done against an Abbott Architect i2000SR. Linear regression over the range of samples included ( 1.0 to $140 \mathrm{IU} / \mathrm{ml}, \mathrm{n}=50$ ) was $\mathrm{y}=0.84 \mathrm{x}-0.64, \mathrm{r}^{2}=0.997$ with a mean bias of $-2.2 \mathrm{IU} / \mathrm{ml}$.

Samples from 3 distributions of the UK NEQAS Tumor marker scheme were also analysed and compared with the All Lab Trimmed Mean. For these samples $(\mathrm{n}=15)$ the mean deviation from the ALTM was $-7.6 \%$ (range $-16.3 \%$ to $+1.31 \%$ ). Samples with AFP IS 72/225 added demonstrated recoveries of $100 \%$ and $98 \%$ at added levels of 20 and $40 \mathrm{kU} / \mathrm{L}$. (Thanks to UKNEQAS, Edinburgh, for permission to publish).

A sample with an approximate AFP value of $170,000 \mathrm{IU} / \mathrm{ml}$ was correctly identified as beyond the measuring range $(325 \mathrm{IU} / \mathrm{ml})$ of the assay when analysed neat, $1 / 10,1 / 100$ and 1/200 dilutions, giving an acceptable result at an on-board dilution of $1 / 1000$.

We conclude that the Olympus AFP assay shows excellent imprecision performance at all levels checked, with no indication of any hook effect up to the level investigated. EQA samples show no problems with either recovery of the international standard or with hepatoma samples. Further work is necessary to confirm the claimed absence of hook effect to levels of AFP greater than 1,000,000IU/ml. 


\section{BIOLOGICAL AND ANALYTICAL VARIATIONS OF CA 15.3 ASSAYS: INFLUENCE ON CUTOFFS AND REFERENCE CHANGE VALUE}

François Mullier, Jérémie Gras, Catherine Fillée, Marianne Philippe

Cliniques Universitaires Saint-Luc, Clinical Biochemistry, Avenue Hippocrate, 10, 1200 Brussels, Belgium

Aims:

Our objective was to evaluate the clinical performance of the Unicel ${ }^{\mathrm{TM}}$ DxI 800 (Beckman Coulter) and the Elecsys ${ }^{\mathrm{TM}} 2010$ (Roche Diagnostics) for CA 15.3 antigen determination.

Method:

A cohort of patients involved in a breast cancer follow-up study (494 patients-601 results) were included and considered as normal if disease free at the time of sampling. Coefficient of agreement $\kappa$, Bland Altman, ROC curves and $\sigma$ level of performance were calculated using the SPSS, JMP program (BA and $\kappa$ ) and Westgard EZRules ${ }^{\mathrm{TM}} 3$ program respectively. Reference change value $(\mathrm{RCV})$ was calculated as followed $\mathrm{RCV}=\sqrt{2} \times \mathrm{Z} \times \sqrt{ } \mathrm{CV}_{\mathrm{A}}{ }^{2}+\mathrm{CV}_{\mathrm{I}}{ }^{2}\left(\mathrm{Cv}_{\mathrm{I}}: 5,7 \%\right.$, Ricos databases/ $\mathrm{Z}=2,58$ (p:99\%)).

Results:

The mean systematic difference was $42 \%$. The highest recurrence detection is obtained with the cut-offs set at $34.1 \mathrm{KU} / \mathrm{L}$ for Elecsys and 22.1 KU/L for DxI. The AUC were 0.882 and 0.827 respectively $(\mathrm{P}<0.001)$ and the $\kappa 0.800$. Positive predictive values (PPV) reached $76.4 \%$ for Elecsys and $67.1 \%$ for DxI. At cut-off levels, $\mathrm{RCV}_{99 \%}$ are $29.8 \%$ (dispersion: $+/-10 \mathrm{KU} / \mathrm{L})$ and $27.7 \%(+/-6.1 \mathrm{KU} / \mathrm{L})$ and $\sigma$ levels reached 2.63 and 3.10 for Elecsys and DxI respectively.

Conclusions :

Optimisation of cut-offs improves PPV of both assays but it remains low, $+/-75 \%$. Each lab must defined their own cutoffs and RCV. The low Six-Sigma level underline the need of further improvements if the desirable $\mathrm{CV}_{\mathrm{A}}(2.9 \%)$ defined by Fraser must be reached.

P5_6

\section{REFERENCE CHANGE VALUES AND DISEASE SPECIFIC CUTOFF FOR CA 125 ASSAYS.}

François Mullier, Jérémie Gras, Catherine Fillée, Marianne Philippe

Cliniques Universitaires Saint-Luc, Clinical Biochemistry, Avenue Hippocrate, 10, 1200 Brussels, Belgium

Aims:

In this work, we compare the clinical performances of two CA 125 assays, the new Unicel ${ }^{\mathrm{TM}} \mathrm{DxI} 800$ and the Elecsys ${ }^{\mathrm{TM}} 2010$.

Method :

395 sera, freshly drawn (286 patients) were run in parallel during six month. If ovarian cancer free at the time of sampling, the patients were considered as normal. Coefficient of agreement $\kappa$, Bland-Altman (BA), ROC curves and $\sigma$ levels of performance were calculated using the SPSS, JMP and Westgard EZRules ${ }^{\mathrm{TM}} 3$ programs, the reference change value $(\mathrm{RCV})$ was calculated as $\mathrm{RCV}=\sqrt{2} \times \mathrm{Z} \times \sqrt{ } \mathrm{CV}_{\mathrm{A}}{ }^{2}+\mathrm{CV}_{\mathrm{I}}{ }^{2}\left(\mathrm{CV}_{\mathrm{I}}=13,6 \%, \mathrm{Z}=1,96(\mathrm{p}: 95 \%)\right)$.

Results

When all patients are considered, the highest accuracy and concordance $(\kappa=0.932)$ were obtained with a cut-off of 68,5 $\mathrm{KU} / \mathrm{L}$ for Elecsys and 87,4 KU/L for DxI with AUCs and $\sigma$ values of $0.84,6,7$ and $0.85,7,52$ respectively $(\mathrm{P}>0.05)$. In ovarian cancer $(n=42)$, the medians were $162 \mathrm{KU} / \mathrm{L}$ for Elecsys and $206 \mathrm{KU} / \mathrm{L}$ for $\mathrm{DxI}(\mathrm{p}<0.05)$, in benign diseases 17 vs $12 \mathrm{KU} / \mathrm{L}(\mathrm{p}<0.05)$, in endometriosis $34 \mathrm{vs} 26 \mathrm{KU} / \mathrm{L}(\mathrm{p}<0.05)$. In the follow-up group (145 results), the highest recurrence detection is obtained with cutoffs of $27,4 \mathrm{KU} / \mathrm{L}$ for Elecsys and $26.7 \mathrm{KU} / \mathrm{L}$ for DxI with AUCs of 0.91 and 0.90 respectively $(\mathrm{P}>0,05)$ and $\kappa=0,956$. The PPV reached $93,5 \%$ for Elecsys and $94 \%$ for DxI whereas the RCV were $+/-10.6 \mathrm{KU} / \mathrm{L}$ and $+/-10.7 \mathrm{KU} / \mathrm{L}$ respectively.

Conclusions.

For both methods having similar performances, our study led to the definition of optimal cutoffs. 
TOTAL PSA WHO CALIBRATION : IMPACT ON DECISION POINT

Catherine Fillée, Pierre Alexandre Olivier, Jean-Michel Allaeys, Marianne Philippe

Cliniques Universitaires Saint-Luc, Clinical Biochemistry,Avenue Hippocrate, 10, 1200 Brussels, Belgium

\begin{abstract}
Aims: The first International Reference Preparation (WHO 96/670) has been made available in an attempt to align PSA results across manufacturers. This study was designed to compare the results of the Access Hybritech PSA assay ${ }^{\mathrm{TM}}$ using both the original Hybritech and the WHO calibrations.

Methods: Imprecision was evaluated on QC materials over 22 days. Correlation between Hybritech and the WHO calibrations was evaluated on 20 spiked samples and on 155 patient specimens using the Deming regression. The analytical agreement, relative sensitivity and relative specificity were calculated from the McNemar change test.

Results: At total PSA levels of $0.82,11.66$ and $68.42 \mathrm{ng} / \mathrm{mL}$ total CVs were $10.17 \%, 4.47 \%$ and $4.83 \%$ with the WHO calibration, $10.03 \%, 3.74 \%$ and $4.64 \%$ with the Hybritech one. When all the values are considered, the Deming regression showed a slope of 0.81 and 0.80 for spiked and patient samples. The same slope was obtained for values below $4.0 \mathrm{ng} / \mathrm{mL}$ or for values below $10 \mathrm{ng} / \mathrm{mL}$. At a cutoff level of $4.0 \mathrm{ng} / \mathrm{mL}$, the McNemar change test showed a percentage of agreement of $92 \%$ in the patient group and of $90 \%$ with the spiked samples. If an optimal decision point of $3.1 \mathrm{ng} / \mathrm{mL}$ is applied for PSA WHO calibration, the percentage of agreement reaches $97 \%$, the relative sensitivity $100 \%$ and the relative specificity $96 \%$.

Conclusions: The WHO calibration results are approximately 20\% lower than those obtained with the original Hybritech calibration over the full range of values implying that lower decision points should be considered.
\end{abstract}

\title{
P5_8
}

\section{PERFORMANCE EVALUATION OF AN AUTOMATED IMMUNOASSAY FOR THE DETERMINATION OF AFP (Alpha-fetoprotein) ON THE OLYMPUS AU3000i'M IMMUNOASSAY SYSTEM}

M. P. Roger ${ }^{1}$, B. Grimaudet de Rochebouët ${ }^{1}$, A. Cherasse ${ }^{1}$, E. Lentwojt ${ }^{1}$

D. Cooney ${ }^{2}$, D. Fitzgerald ${ }^{2}$, S.J.S. Gaston ${ }^{2}$

${ }^{1}$ Olympus Life and Material Science Europa GmBH, Rungis, France

${ }^{2}$ Olympus Life and Material Science Europa GmbH, O'Callaghan's mills, Lismeehan, Ireland

Determination of AFP is used as an aid in the management of patients with non-seminomatous testicular cancer and also in patients with other malignancies such as hepatocellular carcinoma, ovarian, gastrointestinal and pulmonary cancers.

The Olympus AFP assay is a paramagnetic particle, chemiluminescent immunoassay for the quantitative determination of AFP levels in human serum and plasma using the Olympus AU3000i ${ }^{\mathrm{TM}}$ Immunoassay System.

We report here results from our development and evaluation of this automated assay for AFP on the Olympus AU3000i ${ }^{\text {TM }}$ Immunoassay System. This assay is referenced to the WHO $1^{\text {st }}$ International Standard 72/225.

Lowest detectable level was determined to be $0.08 \mathrm{IU} / \mathrm{mL}$.

Assay imprecision was characterized over 20 days (80 reps/instrument) according to CLSI protocol EP5-A. Within run coefficients of variation $(\mathrm{CV})$ for low, medium, and high human serum pools $(1.02,20.5$, and $117 \mathrm{IU} / \mathrm{mL}$ respectively) ranged from 1.4 to $1.7 \%$. Between run coefficients of variation ranged from $1.9 \%$ to $2.8 \%$.

A method comparison with the Roche Elecsys System ${ }^{\circ}$ AFP assay using 97 serum samples ranging from 0.70 to 243.4 $\mathrm{IU} / \mathrm{mL}$ yielded a slope of 0.894 , y-intercept of 0.018 and a correlation coefficient of 0.9982 .

The mean recovery for samples tested for linearity ranged from $92.2 \%$ to $100.1 \%$.

There is no high dose effect at AFP concentrations up to $1800000 \mathrm{IU} / \mathrm{mL}$ and this assay utilizes a two step design which signifcantly reduces the risk of high dose hook effect.

The AU3000i AFP assay demonstrates no significant interference with human proteins such as human alpha2-macroglobulin, human alpha-1-acid glycoprotein, human alpha-1-antitrypsin, human gamma globulin, human serum albumin, human transferrin, human chorionic gonadotropin, and human placental lactogen.

No significant interference was detected from bilirubin, hemolysate and Intralipid. Reagents were also optimised to avoid RF and HAMA interference.

Based on our evaluation, we conclude that the Olympus AFP assay is a sensitive, precise, and specific method which meets the requirements for measuring AFP levels in human serum and plasma. 


\title{
PERFORMANCE EVALUATION OF AN AUTOMATED IMMUNOASSAY FOR THE DETERMINATION OF BR-TC [BR-Tumor Check(CA 153 antigen)] ON THE OLYMPUS AU3000i ${ }^{\mathrm{TM}}$ IMMUNOASSAY $^{-15}$ SYSTEM
}

\author{
M. P. Roger ${ }^{1}$, E. Lentwojt ${ }^{1}$, O. Ilelaboye ${ }^{2}$, D. Cooney ${ }^{2}$, D. Fitzgerald ${ }^{2}$, S.J.S. Gaston ${ }^{2}$ \\ ${ }^{1}$ Olympus Life and Material Science Europa GmBH, Rungis, France \\ ${ }^{2}$ Olympus Life and Material Science Europa GmbH, O'Callaghan's mills, Lismeehan, Ireland
}

Determination of BR-TC is used as an aid in the management of breast cancer patients.

The Olympus BR-TC assay is a paramagnetic particle, chemiluminescent immunoassay for the quantitative determination of BR-TC levels in human serum and plasma using the Olympus AU3000 ${ }^{\mathrm{TM}}$ Immunoassay System.

We report here results from our development and evaluation of this automated assay for BR-TC on the Olympus AU3000 ${ }^{\mathrm{TM}}$ Immunoassay System. This assay is traceable to the Access ${ }^{\circledR}$ BR Monitor assay.

Lowest detectable level was determined to be $0.0068 \mathrm{U} / \mathrm{mL}$.

Assay imprecision was characterized over 20 days (80 reps/instrument) according to CLSI protocol EP5-A. Within run coefficients of variation $(\mathrm{CV})$ for low, medium, and high human serum pools $(9.76,224$, and $421 \mathrm{U} / \mathrm{mL}$ respectively) ranged from 2.6 to $3.9 \%$. Between run coefficients of variation ranged from $4.3 \%$ to $5.5 \%$.

A method comparison with the Beckman, Dxi, BR-Monitor assay using 141 serum samples ranging from 0.20 to 772 $\mathrm{U} / \mathrm{mL}$ yielded a slope of 0.97 , y-intercept of 0.73 and a correlation coefficient of 0.98 .

The mean recovery for samples tested for linearity ranged from $89.1 \%$ to $97.7 \%$.

There is no high dose effect at BR-TC concentrations up to $20000 \mathrm{U} / \mathrm{mL}$ and this assay utilizes a two step design which signifcantly reduces the risk of high dose hook effect.

The AU3000i BR-TC assay demonstrates no significant cross - reactivity with CA 19-9, CA 125, AFP and CEA.

No significant interference was detected from bilirubin, hemolysate and Intralipid. Reagents were also optimised to avoid $\mathrm{RF}$ and HAMA interference.

Based on our evaluation, we conclude that the Olympus BR-TC assay is a sensitive, precise, and specific method which meets the requirements for measuring BR-TC levels in human serum and plasma.

\section{P5_10}

\section{PERFORMANCE EVALUATION OF AN AUTOMATED IMMUNOASSAY FOR THE DETERMINATION OF CEA (Carcinoembryonic Antigen) ON THE OLYMPUS AU3000i' ${ }^{\mathrm{TM}}$ IMMUNOASSAY SYSTEM}

\author{
M. P. Roger ${ }^{1}$, E. Lentwojt ${ }^{1}$, O. Ilelaboye ${ }^{2}$, D. Cooney $^{2}$, D. Fitzgerald ${ }^{2}$, S.J.S. Gaston ${ }^{2}$ \\ ${ }^{1}$ Olympus Life and Material Science Europa GmBH, Rungis, France \\ ${ }^{2}$ Olympus Life and Material Science Europa GmbH, O'Callaghan's mills, Lismeehan, Ireland
}

Determination of CEA is used as an aid in the management of cancer patients and in the assessment of prognosis.

The Olympus CEA assay is a paramagnetic particle, chemiluminescent immunoassay for the quantitative determination of CEA levels in human serum and plasma using the Olympus AU3000i ${ }^{\mathrm{TM}}$ Immunoassay System.

We report here results from our development and evaluation of this automated assay for CEA on the Olympus AU3000i ${ }^{\text {TM }}$ Immunoassay System. This assay is referenced to $1^{\text {st }}$ IRP WHO Reference Standard 73/601.

Lowest detectable level was determined to be $0.125 \mu \mathrm{g} / \mathrm{L}$.

Assay imprecision was characterized over 20 days (80 reps/instrument) according to CLSI protocol EP5-A. Within run coefficients of variation (CV) for low, medium, and high human serum pools $(1.74,29.9$, and $227 \mu \mathrm{g} / \mathrm{L}$ respectively) ranged from 1.4 to $1.5 \%$. Between run coefficients of variation ranged from $2.3 \%$ to $3 \%$.

A method comparison with the Roche Elecsys System ${ }^{\circ}$ CEA assay using 100 serum samples ranging from 0.61 to $497 \mu \mathrm{g} / \mathrm{L}$ yielded a slope of 0.93 , y-intercept of 0.88 and a correlation coefficient of 0.9733 .

The mean recovery for samples tested for linearity ranged from $94.52 \%$ to $105.98 \%$.

There is no high dose effect at CEA concentrations up to $350000 \mu \mathrm{g} / \mathrm{L}$ and this assay utilizes a two step design which signifcantly reduces the risk of high dose hook effect.The AU3000i CEA assay demonstrates an interference of $0.31 \%$ with NCA 1 and $25.31 \%$ with NCA 2 and no significant cross - reactivity with AFP. No significant interference was detected from bilirubin, hemolysate and Intralipid. Reagents were also optimised to avoid RF and HAMA interference.

Based on our evaluation, we conclude that the Olympus CEA assay is a sensitive, precise, and specific method which meets the requirements for measuring CEA levels in human serum and plasma. 
P5_11

\title{
PERFORMANCE EVALUATION OF AN AUTOMATED IMMUNOASSAY FOR THE DETERMINATION OF GI-TC [GI-TUMOR CHECK(CA 19-9 antigen)] ON THE OLYMPUS AU3000i' ${ }^{\mathrm{TM}}$ IMMUNOASSAY SYSTEM
}

\author{
M.P. Roger ${ }^{1}$, B. de Grimaudet de Rochebouët ${ }^{1}$, E. Lentwojt ${ }^{1}$, D. Cooney ${ }^{2}$, D. Fitzgerald ${ }^{2}$, S.J.S. Gaston ${ }^{2}$ \\ ${ }^{1}$ Olympus Life and Material Science Europa GmBH, Rungis, France \\ ${ }^{2}$ Olympus Life and Material Science Europa GmbH, O'Callaghan's mills, Lismeehan, Ireland
}

Determination of GI-TC is used as an aid in the management of patients diagnosed with cancers of the exocrine pancreas.

The Olympus GI-TC assay is a paramagnetic particle, chemiluminescent immunoassay for the quantitative determination of GI-TC levels in human serum and plasma using the Olympus AU3000 ${ }^{\text {TM }}$ Immunoassay System.

We report here results from our development and evaluation of this automated assay for GI-TC on the Olympus AU3000 ${ }^{\mathrm{TM}}$ Immunoassay System.

This assay is traceable to the Access ${ }^{\circledR}$ GI Monitor assay.

Lowest detectable level was determined to be $0.003 \mathrm{U} / \mathrm{mL}$.

Assay imprecision was characterized over 20 days (80 reps/instrument) according to CLSI protocol EP5-A. Within run coefficients of variation (CV) for low, medium, and high human serum pools $(32.7,82.8$, and $1139 \mathrm{U} / \mathrm{mL}$ respectively) ranged from 1.2 to $2 \%$. Between run coefficients of variation ranged from $3 \%$ to $3.5 \%$.

A method comparison with the Beckman, Dxi, GI-monitor assay using 137 serum samples ranging from 1.67 to 2258

$\mathrm{U} / \mathrm{mL}$ yielded a slope of 1.06 , y-intercept of -1.29 and a correlation coefficient of 0.97 .

The mean recovery for samples tested for linearity ranged from $91 \%$ to $112 \%$.

There is no high dose effect at GI-TC concentrations up to $500.000 \mathrm{U} / \mathrm{mL}$ and this assay utilizes a two step design which signifcantly reduces the risk of high dose hook effect.

The AU3000i GI-TC assay demonstrates no significant cross - reactivity with

CA 15 3, CA 125, AFP, PSA and CEA.

No significant interference was detected from bilirubin, hemolysate and Intralipid. Reagents were also optimised to avoid RF and HAMA interference.

Based on our evaluation, we conclude that the Olympus GI-TC assay is a sensitive, precise, and specific method which meets the requirements for measuring GI-TC levels in human serum and plasma. 


\title{
PERFORMANCE EVALUATION OF AN AUTOMATED IMMUNOASSAY FOR THE DETERMINATION OF TOTAL PSA (Total Prostate Specific Antigen) ON THE OLYMPUS AU3000i ${ }^{\mathrm{TM}}$ IMMUNOASSAY SYSTEM
}

\author{
M.P. Roger ${ }^{1}$, C. Camous ${ }^{1}$, A. Cherasse ${ }^{1}$, E. Lentwojt ${ }^{1}$, D. Cooney ${ }^{2}$, D. Fitzgerald ${ }^{2}$, S.J.S. Gaston ${ }^{2}$ \\ ${ }^{1}$ Olympus Life and Material Science Europa GmBH, Rungis, France \\ ${ }^{2}$ Olympus Life and Material Science Europa GmbH, O'Callaghan's mills, Lismeehan, Ireland
}

Determination of PSA is used as an aid in the management of patients with prostate cancer.

The Olympus PSA assay is a paramagnetic particle, chemiluminescent immunoassay for the quantitative determination of PSA levels in human serum and plasma using the Olympus AU3000 ${ }^{\mathrm{TM}}$ Immunoassay System. We report here results from our development and evaluation of this automated assay for PSA on the Olympus AU3000i ${ }^{\mathrm{TM}}$ Immunoassay System.

This assay is referenced to the WHO $1^{\text {st }}$ International Standard 96/670 (Stanford Reference Materials).

Lowest detectable level was determined to be $0.002 \mathrm{ng} / \mathrm{mL}$. Functional sensitivity was $0.02 \mathrm{ng} / \mathrm{mL}$.

Assay imprecision was characterized over 20 days ( 80 reps/instrument) according to CLSI protocol EP5-A. Within run coefficients of variation $(\mathrm{CV})$ for low, medium, and high human serum pools $(1.0,10.6$, and $25.3 \mathrm{ng} / \mathrm{mL})$ ranged from 1.5 to $3.0 \%$. Between run coefficients of variation ranged from $3.0 \%$ to $5.0 \%$.

A method comparison with the Roche Elecsys System ${ }^{\odot}$ PSA assay of 121 serum samples ranging from 0.1 to $83.6 \mathrm{ng} / \mathrm{ml}$ yielded a slope of 0.941 , y-intercept of -0.059 and correlation coefficient of 0.9868 .

The mean recovery for samples tested for linearity ranged from $97.0 \%$ to $107.0 \%$.

There is no high dose effect at PSA concentrations up to $123835 \mathrm{ng} / \mathrm{mL}$ and this assay utilizes a two step design which signifcantly reduces the risk of high dose hook effect.

The AU3000i PSA assay recognizes free PSA and PSA-ACT on an equimolar basis and demonstrates no significant crossreactivity with PAP and ACT. No significant interference was detected from bilirubin, hemolysate and Intralipid. Reagents were optimised to avoid RF and HAMA interference.

Based on our evaluation, we conclude that the Olympus PSA assay is a highly sensitive, precise, and specific method which meets the requirements for measuring PSA levels in human serum and plasma.

\section{P5_13}

\section{THE CLINICAL UTILITY OF ARCHITECT ${ }_{X R}$, IMMUNO-1, AND ELECSYS ASSAYS FOR CA 19-9}

Ulf-Håkan Stenman, Kristina Hotakainen, Pirjo Tanner, Henrik Alfthan and Caj Haglund.

Dept. of Clinical Chemistry, Helsinki University Central Hospital, P.O.B. 700, 00029 HUCH, Finland

\begin{abstract}
Aims: To determine the reference limit of CA 19-9 and compare the clinical usefulness of three assays for diagnosis of gastrointestinal cancer.

Methods: CA 19-9 was determined using the ARCHITECT CA 19-9 XR (ARC), Elecsys, and Immuno-1 (IMM-1) assays in samples from 525 patients undergoing examinations for gastrointestinal disease. Clinical performance of the methods was evaluated by receiver operating characteristic (ROC) curve analysis using 114 samples from patients with benign diseases, 45 with pancreatic or biliary cancer, 69 patients with colorectal or gastric cancer, and 43 with other cancers. The reference limit was defined as the $97.5^{\text {th }}$ percentile of the concentrations in 312 apparently healthy individuals aged 48-70 years. Results: Overall concordance between ARC and the other methods was good with correlation coefficients $(r)$ of 0.93 (ARC-IMM-1; 95\% confidence interval (CI) 0.92-0.94), and 0.89 (ARC-Elecsys; 95\% CI 0.88-0.91), but for values below $100 \mathrm{kU} / \mathrm{L}$, the concordance was poor (ARC-IMM- $1 r=0.36$ and ARC-Elecsys $r=0.57$ ). In benign conditions, the ARC assay gave significantly lower values (mean $35 \mathrm{kU} / \mathrm{L}$ ) than IMM-1 and Elecsys (mean 110 and $80 \mathrm{kU} / \mathrm{L}$, respectively), while the concentrations in cancer patients were fairly similar $(770 \mathrm{kU} / \mathrm{L}$ for ARC; 755 and $300 \mathrm{kU} / \mathrm{L}$ for IMM-1 and Elecsys, respectively). In ROC analysis for detection of pancreatic and biliary cancer the AUC value for the ARC assay was 0.84 ( $<<0.0001)$, for IMM-1 0.55, and 0.62 for Elecsys. The significantly higher AUC values for ARC were a result of lower values in patients with benign disease. For the ARC assay, the median concentration of CA 19-9 in healthy individuals was $4.0 \mathrm{kU} / \mathrm{L}$, and the $95^{\text {th }}$ and $97.5^{\text {th }}$ percentiles were 19.8 and $25.8 \mathrm{kU} / \mathrm{L}$, respectively.

Conclusions: The ARC assay for CA 19-9 provides better differentiation between malignant and benign gastrointestinal disease than the two other methods tested. The reference limit of the ARC assay can be lowered to $26 \mathrm{kU} / \mathrm{L}$ without compromising specificity in comparison with other methods.
\end{abstract}




\title{
CLINICAL IMPACT OF USE OF DIFFERENT PSA ASSAYS IN PROSTATE CANCER SCREENING
}

\author{
Kwiatkowski Maciej, Seiler Daniel, Arndt Brigitte, Gregorin Joel, Huber ${ }^{1}$ Andreas, Recker Franz
}

Clinic of Urology, Kantonsspital Aarau, Aarau, Switzerland, 'Center of Laboratory Medicine, Kantonsspital Aarau, Switzerland

\begin{abstract}
AIMS: The aim of this study was to investigate the clinical implications of using different prostate-specific antigen (PSA) assays in the PSA-based screening for prostate cancer $(\mathrm{PCa})$.

METHODS: 2543 men participating in the prospective screening study for PCa (Swiss site of the ERSPC study) had parallel measurements with Beckman Coulter Access and Abbott AxSym total PSA (t-PSA) assays at the same time. The AxSym assay was calibrated using the WHO calibrator whereas the Access assay was calibrated using the Hybritech calibrator. The cut-off value of $3.0 \mathrm{ng} / \mathrm{ml}$ of t-PSA of Beckman Access has been used as prostate biopsy indication.

RESULTS: Median tPSA was $1.0 \mathrm{ng} / \mathrm{ml}(\mathrm{min} .0 .02-\max .59 .5 \mathrm{ng} / \mathrm{ml}$ ) for AxSym t-PSA and $1.2 \mathrm{ng} / \mathrm{ml}(\mathrm{min} .0 .01$ - max. $48.8 \mathrm{ng} / \mathrm{ml})$ for Access t-PSA respectively. 418 men $(16.4 \%$ of investigated population) had the Access t-PSA $>=3.0 \mathrm{ng} / \mathrm{ml}$ and were offered the biopsy. In this selected group the median tPSA was $3.5 \mathrm{ng} / \mathrm{ml}$ (min. $1.1-\max .59 .5$ $\mathrm{ng} / \mathrm{ml}$ ) for AxSym t-PSA and 4.2ng/ml (min. 3.00 - max. $48.81 \mathrm{ng} / \mathrm{ml}$ ) for Access t-PSA respectively, the PCa detection rate amounted to $23.3 \%$ and the incidence rate to $3.8 \%$. 296 men (11.6\% of investigated population) had the AxSym t-PSA $>=3.0 \mathrm{ng} / \mathrm{ml}$ amounting to PCa detection rate at $25.9 \%$ and incidence rate at 3.0\%). As a consequence $126 \mathrm{men}$ would have been considered "normal" at $3.0 \mathrm{ng} / \mathrm{ml}$ cut-off value if tested with AxSYM as opposed to Access. In this "discrepancy" group PCa detection rate amounted to $18.2 \%$ and incidence rate to $0.8 \%$. Interestingly, only 4 men in the investigated population had experienced the reverse situation having t-PSA AxSym $>=3 \mathrm{ng} / \mathrm{ml}$ whereas t-PSA Access was $<3.0 \mathrm{ng} / \mathrm{ml}$.

CONCLUSIONS: In view of the fact that there is a strong trend to lower PSA cut-off values to recommend prostate biopsy, the interassay variations gain increasing importance. Our study shows that for two assays investigated at the cut-off level of $3.0 \mathrm{ng} / \mathrm{ml}$ this difference can result in $30 \%$ more or less men (or $5 \%$ of total men population in this age) prompted for prostate biopsy and the use of the same cut-off value in an unadjusted manner may result in failure to detect $\mathrm{PCa}$ at an earlier, thus more curable stage. The physician should be aware of these clinical implications to counsel patients appropriately and to decide when to refer for prostate biopsy.
\end{abstract}

\section{P6_2}

\section{RESULTS OF FOLLOW UP USING TUMOR MARKERS AND PET/CT IN COLORECTAL CANCER}

Ludmila Lipska, Vladimir Visokai, Miroslav Levy, Lucie Strupova

Department of Surgery, Thomayer Teaching Hospital and First Faculty of Medicine, Charles University in Prague, Videnska 800, 14059, Prague 4, Czech Republic

Aims: Complete surgical removal of colorectal cancer relapse is still the best treatment, if possible. Resectability depends on the extent of the relapse, surgical approach and patient's general condition. In order to minimize the extent of the relapse, early detection is benefical.

Material and methods: In our database, there are 1300 patients followed up after operation for colorectal cancer. In 235 of them PET or PET/CT were indicated, 105 of these had relapse confirmed and 68 patients were indicated for operation. Surgery with curative intent was performed in 57 cases. Indications for PET or PET/CT were elevation of tumor markers or suspition on ultrasound, chest X-ray or CT scan.

Results: In this cohort of patients with active system of follow up , the resectability of relapse is $55 \%$.

Conclusions: Active follow up and an aggressive surgical approach result in a good resectability rate and improves the patients chances for survival. 
PSA-VELOCITY AND ARTIFICIAL NEURAL NETWORK (ANN)-VELOCITY TO DIFFERENTIATE PROSTATE CANCER FROM BENIGN PROSTATIC DISEASE - RESULTS FROM 10 YEARS OF ROUTINE FREE PSA USE

Carsten Stephan, Nicola Büker, Henning Cammann², Hellmuth-A. Meyer, Stefan A. Loening', Klaus Jung, Michael Lein

Dept. of Urology, Charité - Universitätsmedizin, Charitéplatz 1, 10117 Berlin, GERMANY

Aims: PSA-velocity (PSAV) and also \%free PSA-based artificial neural networks (ANN) have been suggested to enhance the discrimination between prostate cancer (PCa) and benign prostate hyperplasia (BPH) compared with PSA alone. This study is the first to report on the use of ANN-velocity to answer the question if an improved differentiation between PCa and $\mathrm{BPH}$ is possible compared with PSA alone for prostate biopsy decision.

Methods: The study comprised 199 patients with PCa $(n=49)$ or BPH $(n=150)$ with at least three PSA estimations and a minimum of three months intervals between the measurements. The PSA and ANN-velocity - which were calculated with the \%free based ANN "ProstataClass" - were classified into four categories. Group 1 includes the increasing PSA and ANN values, Group 2 the continuous values, Group 3 the decreasing values and Group 4 the inconstant values.

Results: $70 \%$ of PCa-patients have the typical increasing PSAV. In comparison, the ANN-velocity only shows this in $37 \%$ of all PCa-patients. However, BPH-patients benefit from the additional ANN-velocity since the continuous values are significantly more ( $61 \%$ vs. $42 \%$ ) if the ANN-velocity is used instead of the PSA-velocity. This avoids repeated prostate biopsies in approximately $20 \%$ of all BPH-patients.

Conclusions: PSAV has only limited usefulness to detect PCa with only $70 \%$ increasing PSA values while $30 \%$ of all PCa do not have the typical PSAV. The ANN-velocity cannot improve the PCa detection rate but may save approximately $20 \%$ of unnecessary prostate biopsies in known BPH-patients.

\section{P6_4}

\section{PHARMACOKINETIC TRANSLATIONAL STUDY IN ADVANCED SOLID TUMORS OF 5-FU WITH AN ADMIXTURE OF A GALACTOMANNAN DERIVATIVE}

Mark Zalupski ${ }^{1}$, Jyotsna Fuloria ${ }^{2}$, Raymond Perez ${ }^{3}$, Yousif Abubaker ${ }^{4}$, Kenneth D. Nahum ${ }^{5}$, David Platt ${ }^{6}$, Anatole A. Klyosov ${ }^{6}$ Eliezer Zomer ${ }^{6}$

${ }^{1}$ Univ. of Michigan MC, Ann Arbor,MI 48109; ${ }^{2}$ Ochser Clinic, New Orleans, LA 70121; ${ }^{3}$ Florida Oncology Associates, Jacksonville, FL 32207; ${ }^{4}$ Cancer Clinic, Howell, NJ 07731; ${ }^{5}$ Dartmouth Medical Center,Lebanon, NH 03756; ${ }^{6}$ ProPharmaceuticals, Inc., Newton, MA 02459, USA

Aim: A galactomannan derivative (cGM, DAVANAT ${ }^{\circledR}$ ) produced from a plant origin, characterized by exposed galactose that interacts with cancer cell lectins, was tested for its effect on chemotherapy. Non-human, in vivo efficacy studies demonstrated that cGM enhances the anti-neoplastic effects of 5-fluorouracil (5-FU) in human colon tumor explants. The study was designed to elucidate the effect of cGM on 5-FU pharmacokinetics in both animal and humans. The study sought to demonstrate the translation of pharmacokinetic results from animal models to patients with advanced solid tumors.

Methods: Bio-distribution of ${ }^{14} \mathrm{C} 5-\mathrm{FU}\left(108 \mathrm{mg} / \mathrm{m}^{2}\right)$ admixture with $\mathrm{cGM}\left(180 \mathrm{mg} / \mathrm{m}^{2}\right)$ was studied in mice with human colon tumor explants using a regimen similar to Phase I. Patient plasma samples was analyzed by Liquid Chromatography Mass Spectrometry for pharmacokinetic calculation.

Results: In the mouse study, cGM co-administered with 5-FU, significantly increased exposure (AUChr·nCi/g) of 5-FU in tumors by $17 \%(\mathrm{P}<0.05)$, while changes in plasma, lung, kidney, and liver ranged from 10 to $-6 \%$. This data paralleled findings in cancer patients, where regression analysis of the pharmacokinetic parameters revealed an average $40 \%$ increase in 5-FU plasma exposure (AUC) at the highest cGM dose $\left(280 \mathrm{mg} / \mathrm{m}^{2}\right)$. In addition, the plasma half-life increased from $47 \mathrm{~min}$ on day 1 to $90 \mathrm{~min}$ on day 4, as compared to those reported in the literature for 5-FU bolus regimen (5 to $22 \mathrm{~min}$ ).

Conclusion: The pharmacokinetic profile of 5-FU co-administered with cGM in the mouse model has translated well in patients with solid tumors. The patient data revealed varied increases of 5-FU for individual patients with no corresponding effects on safety measurements. These findings warrant further investigation in order to determine whether the increased efficacy of 5-FU with cGM, as observed in the pre-clinical studies, will translate into clinical benefits for patients.

Acknowledgements: The authors are extremely grateful to Dr. Marilyn Pike and the hospital staffs who made the study successful. 
P6_5

PONS-S TRIAL (PATIENT ORIENTED SURVEILLANCE-STUDY): INTENSIFIED FOLLOW-UP OF BREAST CANCER PATIENTS BASED ON TUMOR MARKER KINETICS AND HIGH-END IMAGING

\author{
Petra Stieber ${ }^{1}$, Richard Paul Baum², Ursula Goldmann-Posch³, Volker Heinemann ${ }^{4}$ \\ ${ }^{1}$ Institute of Clinical Chemistry, Klinikum der Universität München-Großhadern-, Marchioninistraße 15, \\ 81377 München, Germany
}

${ }^{2}$ Department of Nuclear Medicine, Zentralklinik Bad Berka, Germany, ${ }^{3}$ PONS-S Foundation (Patient OrieNted Surveillance-Study) Kulmain, Germany, ${ }^{4}$ Medical Department III, Klinikum der Universität München -Großhadern, Germany

\begin{abstract}
Aims:
At the present time, updated scientific evidence supporting intensified follow-up of breast cancer patients is nonexistent. To the greatest part, this is due to two trials performed two decades ago which failed to demonstrate a survival benefit when intensified follow-up (without testing of tumor markers) was compared to a conventional procedure. However, the clinical relevance of these trials needs to be questioned. By todays' standards they used outdated diagnostic and therapeutic approaches. The PONS-S Foundation (Patient OrieNted Surveillance-Study) was initiated by patients and researchers with the aim to provide a scientific basis for modern follow-up strategies for breast cancer and other cancers. PONS-S wants to bridge the capabilities of oncological biomarkers and all aspects of modern imaging technology with more quality of life and quality of survival in the follow-up of women with breast cancer.
\end{abstract}

\title{
Methods:
}

With the exception of metastatic breast cancer and patients suffering from a second tumor all breast cancer patients irrespective of the tumor stage and prognosis can be included in this study. Four weeks after the end of the primary treatment (surgery + radio/chemotherapy) the individual baseline values of CEA (Abbott, AxSym) and CA 15-3 (Roche, Elecsys) of the breast cancer patients have to be assessed. The interpretation is based upon these individual baseline values. A reproducible increase $>=100 \%$, corresponding to a specificity of $100 \%$, is the indicator for metastatic disease. Subsequently, biomarkers are assessed at 6-week intervals. In - addition to that procedure a CT scan of thorax and abdomen is performed every 6 months. At the defined increase of CEA or CA 15-3, a PET-CT is performed in case of a negative CT. If imaging reveals metastatic disease specific treatment is started according to predefined treatment algorhythms.

\section{Summary:}

The diagnostic strategy of the PONS-S trial is designed to detect recurrent disease in an asymptomatic state when it is still amenable to locoregional procedures or to well tolerated innovative systemic long-term treatment. While it is unclear if this strategy has a positive effect on survival, it appears probable that early treatment in an asymptomatic state may help to maintain quality of life and increase the quality of survival. 
BIOCHEMICAL STUDY OF THE BREAST CYSTIC FLUID AND CANCER RISK

\author{
${ }^{1}$ Buda Francesco ${ }^{2}$ Aragona Pietro, ${ }^{3}$ Buda Concetta ${ }^{4}$ Messina Givanna \\ ${ }^{1}$ MM ${ }^{1}$ Medical Oncology and Prevention, City of Udine Hospital, Udine, Italy; ${ }^{2}$ Clinical Research Laboratory and \\ Pathology, S.M.Battuti Hospital, Pordenone, Italy, ${ }^{3}$ Dpt of Obstetrics \& Gynecology,University Messina, Messina, \\ Italy, ${ }^{4} \mathrm{Dp}$ of Oncological Radiotherapy, University Messina, Messina, Italy
}

The relationship of fibrocystic breast disease (FCD) to breast cancer is a subject that has been studied extensively but remains unsettled. Several have attempted to achieve greater specificity by separately evaluating forms or pathological components of the fibrocystic breast disease complex and clarifying the nature of those showing an increased association with cancer. Gross cystic disease is thought to represent an advanced form of the fibrocystic breast disease. Clinically evident macrocysts $(>1 \mathrm{~cm})$ develop mainly in the premenopausal decade and are thought to originate from aprocrine metaplastic lobules. Cystes are not considered premalignant lesions. Several reports, however, indicate that patients with gross cystic disease have a two fourfold higher risk of developing cancer in the ipsilateral or controlateral breasts.Cysts may be viewed as an outcome of sustained aberrations in the microenviroment of the terminal duct lobular units, eventually leading to hypersecretion of fluid. Biochemistry of the breast cyst fluid obtained by needle aspiration is of special interest. This medium contains a wide variety of substances, a number of which are not found in the blood of healthy women. Chemical components of breast cyst fluid that have been studied include electrolytes, proteins, growth factors, tumorassociated antigens, enzyme and hormones. Some molecules are a peculiar product of the apocrine cells. The functional stage and /or turnover rate of the apocrine cells in the lining epithelium are different in cystcs categorized as Type I or Type II because of their cationic composition. Type I and Type II cysts also have different amounts of androgen and estrogen conjugates, different degrees of epithelial hyperplasia in the surrounding tissue and conceivably different meaning in relation to cancer risk. In spite of recent progress, there are many gaps in our understanding of the progress of gross cystic disease-related lesions in the premenopausal breasts.In any event, chemical examinations of the breast cyst fluid should be performed routinely. If large-scale follow-up studies will substantiate the emerging opinion that Type I and Type II cysts differ in risk for the subsequent development of cancer, categorization of patients could be of special value.

\title{
P7_2
}

\section{INFLUENCE OF HHV-6 AND HHV-7 ACTIVATION ON IMMUNOLOGICAL PARAMETERS IN COLORECTAL CANCER PATIENTS}

$\underline{\text { Simona Donina }}^{1,2}$, Svetlana Chapenko ${ }^{1}$, Alina Sultanova ${ }^{1}$, Inta Jaunalksne ${ }^{1,3}$, Ludmila Engele ${ }^{2}$, Modra Murovska $^{1}$

${ }^{1}$ August Kirchenstein Institute of Microbiology and Virology, Rigas Stradina University, Riga, Latvia

${ }^{2}$ Riga Eastern Hospital, Latvian Oncology Center, 4 Hipokrata Str., Riga, LV-1079, Latvia

${ }^{3}$ P.Stradina Clinical University Hospital, 13 Pilsonu Str., Riga, Latvia

\begin{abstract}
Aim: Evaluation of influence of HHV-6 and HHV-7 activation on immunological parameters of patients with various stages of CRC.

Materials and Methods: Peripheral blood leukocytes (PBL) and cell free plasma samples were obtained from 47 patients with CRC. Nested polymerase chain reaction (nPCR) was used for the detection of latent (viral genomic sequences in PBL DNA) and active (plasma viremia) viral infection. Immunological parameters (lymphocyte subsets: CD3+, CD4+, CD8+, CD16+, CD95+) were determined by laser flow-cytometry. Level of TNF- $\alpha$ was detected using ELISA kit.

Results: Latent beta-herpesviruses infection was found in $87.2 \%(41 / 47)$ of patients. Frequency of virus activation was three time higher in patients in III-IV stages in comparison to the I-II stages of the disease $(3 / 19,15.8 \%$ and $9 / 22$, $40.9 \%$, respectively). In the patients with active viral infection decline of lymphocyte count: $\mathrm{CD} 3+, \mathrm{CD} 4+(\mathrm{p}<0.005)$ and $\mathrm{CD} 16+$ and increase of CD95 $+(\mathrm{p}<0.01$ in II patients group only) was detected. The decrease of CD4+/CD8+ ratio was revealed in both groups. The level of TNF- $\alpha$ was significantly higher in I and II patients groups with active viral infection $(160.0 \pm 90.3$ and $362.5 \pm 149.25$, respectively) in comparison with the level in patients with latent infection ( $54.9 \pm 30.7$ and $124.2 \pm 81.2$, respectively).

Conclusion: Activation of HHV-6 and/or HHV-7 may increase depression of inductor phase of cellular immune response leading to the decrease of immunoregulatory index. Estimation of the virus-associated impairment of immunological functions and antiviral therapy effect is required for clinical prognosis in patients with colorectal cancer.
\end{abstract}


ATTRACTIN AND DP4 ACTIVITY

Daniel Friedrich, Torsten Hoffmann, Joachim Bär, Michael Wermann, Susanne Manhart, Ulrich Heiser and Hans-Ulrich Demuth

Probiodrug AG, Weinbergweg 22, 06120 Halle, Germany

Aims: Attractin seems to be involved in regulation of a number of physiological processes. It was demonstrated, for instance by overexpression studies and loss of function mutations, that the protein has an influence on the immune status, pigmentation, development of cancer and the neuronal architecture. Nevertheless, the molecular mechanisms of how attractin is integrated in these processes are not understood as yet. One of the most controversially discussed function of attractin is the modulation of the action of bioactive peptides by a protease activity similar to dipeptidyl peptidase 4 (DP4). The aim of our study was to purify the DP4-like activity from human plasma, which was described as attractin and to characterize it in comparison to human DP4.

Methods: The purified protein was characterized with respect to its size, electrophoretic behaviour and binding as well as its kinetic properties. Furthermore, different recombinant isoforms of human attractin were expressed and characterized.

Results: By an over 14000-fold enrichment of DP4-like activity from human plasma we achieved an apparent homogenous attractin fraction. The purified attractin protein and its containing DP4-like activity share the same electrophoretic properties and could be clearly distinguished from different forms of DP4 by electrophoretic methods. In contrast to that, the kinetic characterization of the DP4-like activity of attractin fractions reveals nearly identical properties compared to DP4. Furthermore, after upscaling a DP4 impurity could be demonstrated in purified plasma attractin and the activity could be removed by ADA affinity chromatography, resulting in a homogenous attractin preparation without DP4 activity. These results are substantiated by expression of different attractin isoforms, in which no DP4 activity was found either. Conclusions: Summarizing our data we exclude a DP4-like activity of human attractin. This indicates that the multidomain protein attractin acts as a receptor or adhesion protein rather than a protease.

P7_4

\section{DIPEPTIDYL PEPTIDASE 4 AND FIBROBLAST ACTIVATION PROTEIN ALPHA AS TUMOR SUPPRESSOR GENES IN SOFT TISSUE SARCOMAS}

Joerg Stork ${ }^{1}$, Thomas Greither ${ }^{2}$, Torsten Hoffmann ${ }^{1}$, Jens Rahfeld ${ }^{1}$, Hans-Ulrich Demuth ${ }^{1}$, Helge Taubert ${ }^{2}$, Astrid Kehlen $^{1}$

${ }^{1}$ Probiodrug AG, Weinbergweg 22, 06120 Halle, Germany

${ }^{2}$ Institute of Pathology, Martin Luther University Halle-Wittenberg, Halle, Germany

Dipeptidyl peptidase 4 (DP4) and fibroblast activation protein-alpha (FAP-alpha) are serine proteases with post-prolyl peptidase activities that are discussed as modifier of tumor progression. To study the functional effects of both proteases, stably transfected HEK293 cells expressing the enzymatic active and inactive forms of DP4 as well as of FAP were established and characterized concerning proliferation, motility, adhesion, and invasion. DP4 and FAP overexpression reduced the cell motility, the adhesion to fibronectin, but did not influence the proliferation. Both DP4 and FAP increased the E-Cadherin expression, a molecule involved in adhesion between epithelial cells. DP4 transfection reduced the number of colonies by $60 \%$ in a soft agar assay whereas FAP did not. DP4- and FAP-overexpressing HEK293 cells were xenografted in NOD/SCID mice. The most consistent and fastest tumor development was noticed in the mice xenografted with HEK293 cells expressing the enzymatic inactive DP4. Furthermore, the DP4 and FAP RNA levels in samples of 69 patients with soft tissue sarcoma were analysed by quantitative RT-PCR. In a multivariate Cox Regression analysis, patients with a low FAP mRNA expression have a 4.5 times higher risk to die of their soft-tissue sarcoma (STS) in comparison to patient with a higher FAP mRNA expression $(p=0.017)$. Otherwise, DP4 expression showed only non significant effects on the survival on the patients (low expression: $R R=3.0 ; p=0.103$; medium expression: $R R=2.076$; $\mathrm{p}=0.182$ ). In cross-tables, a significant correlation between DP4 mRNA expression and FAP mRNA expression was determined $(\mathrm{p}=0.026)$. Performing a multivariate Cox Regression analysis with DP4 mRNA expression as a covariant, the relative risk of dying of their tumor for patients with low FAP mRNA expression was 5.1 times higher than for patients with higher FAP expression $(p=0.012)$.

In summary for the first time we could show that DP4 and FAP function as tumor suppressor genes in STS. 


\section{STUDY OF BINDING OF PLATINUM BASED CYTOSTATICS TO DNA STRUCTURE; INFLUENCE OF GLUTATHIONE}

Ondrej Zitka1,2, Dalibor Huska ${ }^{1}$, Sona Krizkova ${ }^{1}$, Vojtech Adam ${ }^{1}$, Tomas Eckschlager ${ }^{3}$, Jan Hrabeta ${ }^{3}$, Jiri Kukacka ${ }^{4}$, Richard Prusa ${ }^{4}$, Libuse Trnkova ${ }^{5}$, Ales Horna ${ }^{6}$, Miroslava Beklova ${ }^{7}$, Rene Kizek $^{1}$

${ }^{1}$ Department of Chemistry and Biochemistry, Mendel University of Agriculture and Forestry, Brno, Czech Republic

${ }^{2}$ Department of Biochemistry, and ${ }^{4}$ Department of Chemistry, Faculty of Science, Masaryk University, Brno, Czech

Republic

${ }^{3}$ Department of Paediatric Haematology and Oncology, and ${ }^{4}$ Department of Clinical Biochemistry and

Pathobiochemistry, 2nd Faculty of Medicine, Charles University, Prague, Czech Republic

${ }^{6}$ Department of Food Engineering and Chemistry, Tomas Bata University, Zlin, Czech Republic

${ }^{7}$ Department of Veterinary Ecology and Environmental Protection, University of Veterinary and Pharmaceutical

Sciences, Brno, Czech Republic

Although platinum based cytostatic drugs have been successfully used in the chemotherapy of cancer for more than 30 years, its biochemical mechanism of action is still unclear. The current accepted opinion about their mechanism of action is that the drugs induce their cytotoxic properties through binding to the nuclear DNA and subsequent interference with normal transcription, and/or DNA replication mechanisms. Nevertheless a cell has several mechanisms to prevent them from binding to the nuclear DNA. The main aim of this work was to investigate the affect of cisplatin binding to the nuclear DNA in the presence of glutathione (GSH), which can be considered as one of the possible molecules connected with the development of drug resistance. Primarily we attempted to study the interactions between cisplatin and GSH using flow injection analysis with electrochemical detection (FIA-ED). The detecton limit for GSH was $100 \mathrm{pg} \mathrm{mL}^{-1}$. It clearly follows from the results obtained that the optimized technique is suitable to investigate the interaction between GSH and cisplatin. Moreover, we evaluated the formation of the complex by spectrometry. The spectrometric results obtained were in good agreement with electrochemical ones. After that we suggested the experimental scheme, where the mixture of GSH, DNA and cisplatin has been kept several hours under specific experimental conditions. The products of this interaction was analysed using various analytical techniques, electrochemical biosensor to detect Pt-DNA adducts, FIA-ED to analyse Pt-GSH complexes and liquid chromatography with mass spectrometry to evaluate the results obtained by the electrochemical techniques.

Acknowledgment: This work was supported by grants from GAAV IAA401990701 and MSMT 6215712402. 
PARAMAGNETIC BEADS COUPLED WITH ELECTROCHEMICAL DETECTION AS A TOOL TO INVESTIGATE TRANSCRIPTOME

Dalibor Huska $^{1,2}$, Sona Krizkova ${ }^{1}$, Vojtech Adam ${ }^{1}$, Jaromir Hubalek ${ }^{3}$, Libuse Trnkova ${ }^{4}$, Richard Prusa ${ }^{5}$, Ladislav Havel$^{2}$, Rene Kizek ${ }^{1}$

${ }^{1}$ Department of Chemistry and Biochemistry, and ${ }^{2}$ Department of Plant Biology, Mendel University of Agriculture

and Forestry, Brno, Czech Republic

${ }^{3}$ Department of Microelectronics, University of Technology, Brno, Czech Republic

${ }^{4}$ Department of Chemistry, Faculty of Science, Masaryk University, Brno, Czech Republic

${ }^{5}$ Department of Clinical Biochemistry and Pathobiochemistry, $2^{\text {nd }}$ Faculty of Medicine, Charles University,

Prague, Czech Republic

Paramagnetic beads are mono-dispersive, polymerous particles which have a stable and defined surface enabling adsorption and/or linking of different bio-reactive molecules. In addition it is known that each mRNA molecule contains sequence of twenty five adenines. This feature can be used for binding of mRNA on the surface of the beads coated by thymine chains. The present work reports on suggesting and optimizing of mRNA separation and detection from biological material via paramagnetic beads coupled with electrochemical detection. Prior to use the beads have to be washed by buffers with various ionic strength and $\mathrm{pHs}$. Based on the results obtained phosphate buffer $(50 \mathrm{mM}, \mathrm{pH} 6.85)$ is suitable for the washing of the beads. Particularly, we suggested an automated procedure including three cycles as follows: 2 min shaking, $30 \mathrm{~s}$ centrifugation and removing of redundant solution. Then, hybridizing solution was added to the washed beads. The hybridized mRNA was released from paramagnetic beads in the presence of $100 \mathrm{mM}$ of sodium perchlorate at $85^{\circ} \mathrm{C}$ for five minutes. The isolated mRNA was subsequently analysed by cyclic voltammetry in acetate buffer. Recovery of isolation of mRNA was about $30 \%$. The paramagnetic beads in connection with an electrochemical sensor can be a promising and convincing tool for the isolation of mRNA as a marker of various illnesses including cancer. Moreover, this technique is less time and chemical consuming than phenol-chloroform extraction.

Acknowledgment: This work was supported by grants from GAAV IAA401990701 and A100040602, and INCHEMBIOL MSMT 0021622412.

P7_7

\title{
SONODYNAMIC THERAPY OF CANCER USING A NOVEL PORPHYRIN DERIVATIVE, DCPH-P-NA(I), WHICH IS DEVOID OF PHOTOSENSITIVITY
}

Masahide Kuroki ${ }^{1}$, Ken Hachimine ${ }^{1}$, Hirotomo Shibaguchi ${ }^{1}$, Motomu Kuroki ${ }^{1}$, Yoshinori Nakae ${ }^{2}$, Ryuji Asano ${ }^{2}$ and Isao Sakata ${ }^{2}$

${ }^{1}$ Department of Biochemistry, Faculty of Medicine, Fukuoka University, Fukuoka,

${ }^{2}$ Photochemical Co., Okayama, Japan

\begin{abstract}
Aim: To produce a new porphyrin derivative that is activated by ultrasound but not by light in order to reduce the skin sensitivity.

Method: We developed a novel porphyrin derivative designated DCPH-P-Na(I) and investigated its photochemical characteristics and sonotoxicity on tumor cells.

Results: DCPH-P-Na(I) exhibited a minimum fluorescent emission by excitation with light in comparison to a strong emission from ATX-70, which is known to reveal both photo- and sonotoxicity. According to this observation, when human tumor cells were exposed to light in the presence of DCPH-P-Na(I) in vitro, the least phototoxicity was observed in contrast to the strong phototoxicity of ATX-70. However, DCPH-P-Na(I) exhibited a potent sonotoxicity on tumor cells by irradiation with ultrasound in vitro. This sonotoxicity was reduced by the addition of L-histidine, but not D-mannitol, thus suggesting that singlet oxygen may be responsible for the sonotoxicity of DCPH-P-Na(I). DCPH-P-Na(I) demonstrated significant sonotoxicity against a variety of cancer cell lines derived from different tissues. In addition, in a mouse xenograft model, a potent growth inhibition of the tumor was observed by sonication after the administration of DCPH$\mathrm{P}-\mathrm{Na}(\mathrm{I})$ to the mouse.

Conclusion: These results suggest that sonodynamic therapy with DCPH-P-Na(I) may therefore be a useful clinical treatment for cancers located deep in the human body without inducing skin sensitivity, which tends to be a major side effect of photosensitizers.
\end{abstract}


THE ROLE OF KRÜPPEL-LIKE FACTOR 6 (KLF6) IN DIFFERENTIATION OF EMBRYONIC STEM CELLS INTO HEPATOCYTES

\author{
Nobuyuki Matsumoto $^{1,2}$, Xiao Zhao ${ }^{2}$, Scott L. Friedman ${ }^{2}$, Fumio Itoh ${ }^{1}$ \\ ${ }^{1}$ Division of Gastroenterology and Hepatology, St. Marianna University School of Medicine, Kanagawa, Japan. \\ ${ }^{2}$ Division of Liver Diseases, Mount Sinai School of Medicine, New York, NY, U.S.A.
}

Background: Krüppel-like factor 6 (KLF6), a zinc finger transcription factor and tumor suppressor gene, plays an important role in regulating proliferation and development. Our previous analysis of $\mathrm{Klf}^{-/}$mice indicates that they are embryonic lethal by embryonic day (E) 12.5, and exhibit markedly reduced hematopoiesis and angiogenesis; they also lack a definable liver (Blood 2006, 107:1357-6). Consistent with this phenotype, Klf6 $\%$ embryonic stem (ES) cells generated by homologous recombination also display significant hematopoietic defects following differentiation into embryoid bodies (EBs). In order to further characterize the role of KLF6 in liver development, our aim was to assess the capacity of $K_{l f 6^{-/}}$ES cells to differentiate into a hepatic lineage, compared to $\mathrm{Klf}^{+/+}$and $\mathrm{Klf}^{+/ /}$ES cells. Methods/Results: $\mathrm{Klf}^{+/+}$ and $\mathrm{Klf}^{+/-}$mouse ES cells were first differentiated into an endodermal population in serum-free conditions using activin A, as confirmed by analysis of Hnf $3 \beta / F$ oxa 2 and brachyury mRNA expression. Next, FGF and BMP4 were added, and expression of hepatic differentation markers was quantified by real time PCR (qRT-PCR) until day 12. The differentiated $\mathrm{Klf}^{+++}$and $\mathrm{Klf}^{+/}$ES cells both expressed endoderm markers, as well as early (e.g., alpha fetoprotein, transthyretin), and late (albumin) hepatic mRNAs. Differentiation was accompanied by a biphasic increase in Klf6 mRNA on day two and again after day four. In contrast, $K l f 6^{-\curvearrowright}$ ES cells displayed significantly reduced expression of endoderm markers, including $\mathrm{Hnf} 3 \beta$, Gata4, Sox 17, and $\mathrm{CxCr}$, a marker for definitive endoderm, and did not express hepatocyte markers (alpha fetoprotein, transthyretin, albumin). Furthermore, forced expression of KLF6 at specific time points using a tetinducible system increased endoderm and hepatic marker expression. Conclusions: KLF6 regulates endoderm formation and hepatic differentiation in a temporally-specific manner in ES cells. These results reveal a novel transcription factor involved in the highly regulated process of liver differentiation.

\title{
P7_9
}

\section{SEARCHING URINARY TUMOR MARKERS FOR BLADDER CANCER USING A TWO DIMENSIONAL DIFFERENTIAL GEL ELECTROPHORESIS (2D-DIGE) APPROACH}

Orenes-Piñero, Esteban, ${ }^{(2)}$ González-Peramato, Pilar, ${ }^{(1)}$ Cortón, Marta, ${ }^{(3)}$ Casal, Ignacio, ${ }^{(4)}$ Serrano, Antonio, ${ }^{(5)}$ Algaba, Ferrán, ${ }^{(1)}$ Sánchez-Carbayo, Marta.*

${ }^{(1)}$ Tumor Markers Group, Molecular Pathology Program, Spanish National Cancer Center, Madrid; (2)Pathology

Department, Hospital de Guadalajara; ${ }^{(3)}$ Biotechnology Program, Spanish National Cancer Center, Madrid;

${ }^{(4)}$ Urology Department, Hospital de Guadalajara; ${ }^{(5)}$ Pathology Department, Fundació Puigvert, Barcelona, Spain

AIMS: In order to identify specific biomarkers for bladder cancer, we evaluated a 2D-DIGE approach for a more comprehensive exploration of the urinary proteome.

METHODS: Using 2D-DIGE analysis, urinary protein extracts belonging to non-neoplastic and bladder tumor cases were labelled with fluorescent dyes. Samples were run using immobilized $\mathrm{pH}$ gradient strips for isoelectric focusing (pH: 3-10, NL, 14x16 cm) and standard continuous SDS-PAGE for the second dimension. Proteins were captured and visualized using a fluorescence scanner (Typhoon 9400). Computational analyses of DIGE images were performed with the DeCyder software. Differentially expressed proteins of 2D-PAGE visualized by SYPRO Ruby staining were excised with an Ettan-Picker and digested with trypsin. Identification or proteins was performed on a 4800 MALDI- TOF/TOF analyzer. The relevance of identified proteins in tumor progression was assessed by immunohistochemistry on tissue microarrays ( $\mathrm{n}=173)$.

RESULTS: Initial observations of 2D-DIGE gels identified over 2,000 spots differentially expressed between nonneoplastic versus patients with bladder cancer, with an increase of $9.9 \%$ proteins in bladder cancer urinary specimens. Computational analyses of the images revealed changes in abundance of tumor vs control spots volume ratios within the 95th confidence level (t-test; $\mathrm{p}<0.05)$. 2D-DIGE combined with MALDI-TOF peptide mass fingerprinting identified 20 proteins differentially expressed between neoplastic and normal urinary specimens. Among the identified proteins, immunohistochemical patterns of Keratin-10 were found to be significantly associated with tumor grade $(\mathrm{p}=0.018)$ and tumor stage $(\mathrm{p}=0.019)$ in bladder cancer tumors. 
CONCLUSIONS: The 2D-DIGE proteomic strategy identified urinary proteomic patterns indicative of the presence of bladder cancer. The current data strongly supports the value of 2D gels profiling of urine in combination with MALDITOF as a biomarker discovery approach for bladder cancer detection. Moreover, keratin-10 was associated with bladder cancer progression.

P7_10

\title{
GALECTIN-3 EXPRESSION IS ASSOCIATED WITH BLADDER CANCER PROGRESSION AND CLINICAL OUTCOME
}

Giacomo Canesin ${ }^{1}$, Pilar Gonzalez-Peramato ${ }^{2}$, Joan Palou ${ }^{3}$, Manuel Urrutia ${ }^{4}$, Carlos Cordón-Cardo ${ }^{5}$ and Marta Sanchez-Carbayo $^{1 *}$

${ }^{1}$ Spanish National Cancer Research Center, Tumor Markers Group, Madrid, Spain

${ }^{2}$ Hospital de Guadalajara, Pathology, Guadalajara, Spain

${ }^{3}$ Fundació Puigvert, Barcelona, Spain

${ }^{4}$ Hospital de Salamanca, Urology Department, Salamanca, Spain

${ }^{5}$ Memorial Sloan-Kettering Cancer Center, NY, USA

\begin{abstract}
Aims: Galectin-3 belongs to a family of carbohydrate-binding proteins characterized by a high affinity for $\beta$-galactosides and a conserved amino-acid sequence. Its function is largely unknown, but a body of evidence suggests its role in cell-cell and cell-matrix interaction, adhesion and apoptosis in certain solid tumors. In the present work, we aim to investigate galectin-3 expression in bladder cancer.

Methods: In a series of 157 bladder tissues, including normal urothelium, superficial lesions and invasive tumors, galectin-3 gene expression was assessed by transcript profiling (U133A). Independent series of primary bladder tumors spotted on tissue arrays $(n=389)$ served to evaluate the associations of immunohistochemical protein expression patterns of galectin-3 with proliferation (Ki67), apoptosis (apopdetek) and clinicopathological variables. Galectin-3 protein levels were quantified in 148 urinary specimens of patients with bladder cancer and controls by enzymeimmunoanalysis.

Results: Galectin-3 gene expression levels increased in invasive tumors as compared to superficial lesions, and was also associated with poor survival $(\mathrm{p}<0.05)$. Immunohistochemical galectin-3 protein expression patterns on tissue arrays were also associated with tumor stage, and clinical outcome $(\mathrm{p}<0.001)$. Moreover, protein expression patterns correlated with proliferative and apoptotic levels $(\mathrm{p}<0.05)$. Furthermore, urinary levels of galectin-3 segregated bladder cancer patients from controls with high diagnostic accuracy $(\mathrm{AUC}=0.73)$.

Conclusions: Tumor transcript levels and protein patterns of galectin-3 were differentially expressed along bladder cancer progression. Urinary protein levels served to identify patients with bladder cancer. These observations suggest a role for galectin-3 as a biomarker for bladder cancer diagnostics, staging and outcome prognosis.
\end{abstract}




\section{CONTENT}

PL01

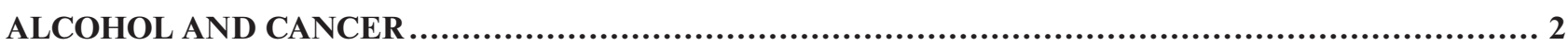

PLO2

THERAPEUTIC STRATEGIES FOR CANCER STEM CELLS ......................................... 2

PL03

A PERSONALIZED APPROACH TO CANCER TREATMENT: HOW BIOMARKERS CAN HELP........ 3

PL04

ATTRACTIN, NEURODEGENERATION AND GLIOMA DEVELOPMENT ............................... 3

PL05

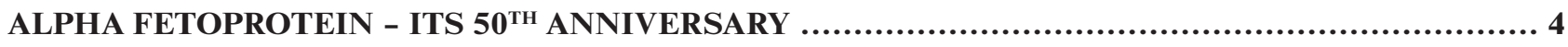

PL06

FINDINGS OF MALIGNANT TUMORS IN EUROPE AND ANCIENT EGYPT IN A DIACHRONIC

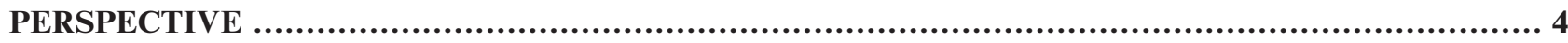

S1_A1

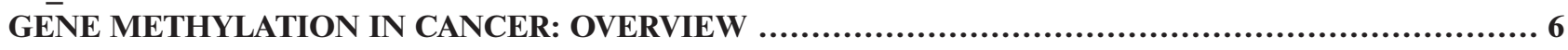

S1_A2

METHYLATED GENES AS PROGNOSTIC AND PREDICTIVE MARKERS IN BREAST CANCER ..... 6 S1_A3

SERUM ASSESSMENT OF GENE PROMOTER REGION METHYLATION FOR THE EARLY

DETECTION OF PROSTATE CANCER

S2_A1

ORIGIN AND EVOLUTION OF CHILDHOOD LEUKEMIA

8

S2_A2

ROLE OF C-KIT GENE IN CORE BINDING FACTOR LEUKEMIA: FROM THE MUTATIONS TO

THE PATHWAYS AND THERAPEUTICS

S2_A3

THE PRINCIPLE ROLE OF ILLEGITIMATE IG REARRANGEMENTS IN PATHOGENESIS

OF B-CELL MALIGNANCIES

S2_A4

COMPLEX DIAGNOSTIC APPROACH TO NON-HODGKIN LYMPHOMAS IN HANDS OF

PATHOLOGISTS AND MOLECULAR BIOLOGISTS

S2_A5

MOLECULAR CYTOGENETICS IN DIAGNOSTICS OF LEUKEMIA

S2_A6

PAX5 PROMOTES LYMPHOMAGENESIS THROUGH THE STIMULATION OF B-CELL

RECEPTOR SIGNALING

S3_A1

PROGNOSTIC SIGNIFICANCE OF CYTOKERATINS - TPS, TPA - IN BREAST CANCER 11

S3_A2

ONCOLOGICAL BIOMARKERS IN THE EARLY DETECTION OF METASTATIC BREAST CANCER...11

S3_A3

CYTOKERATINS AS MARKERS FOR COLORECTAL CARCINOMA

.12

S3_A4

CLINICAL APPROACH OF CYTOKERATINS IN PATIENTS WITH LUNG CANCER 12

S3_A5

PROGNOSTIC FACTORS IN OVARIAN CANCER 13

S3_A6

USING SERUM CYTOKERATIN-18 ASSAYS TO DETERMINE CELL DEATH MODE .13 
S4_A1

HUMAN CHORIONIC GONADOTROPIN (HCG) ISOFORMS AND THEIR EPITOPES:

PHYSIOLOGICAL OCCURRENCE AND DIAGNOSTIC ROLES IN PREGNANCY

AND ONCOLOGY

S4_A2

HCG STANDARDIZATION AND QUALITY CONTROL

S4_A3

HCG IN TESTICULAR AND OTHER MALIGNANT DISEASES

S4_A4

HCG IN DIAGNOSIS AND MANAGEMENT OF GESTATIONAL TROPHOBLASTIC

DISEASE (GTD)

S4_A5

VARIOUS ISOFORMS OF HCG IN CANCER PATIENTS

S5_A1

THE TKTL1 GLUCOSE METABOLISM AND MITOCHONDRIA-INDEPENDENT ENERGY

PRODUCTION IN CANCER CELLS

S5_A2

PATHOLOGY AS THE BASIS FOR A NOVEL TARGETED THERAPY

S5_A3

CLINICAL RELEVANCE OF TKTL1 EXPRESSION IN TUMORS

S5_A4

METABOLIC GROWTH CONTROL OF HUMAN TKTL1-POSITIVE TUMORS IN NUDE MICE WITH

A KETOGENIC DIET

S6_A1

PLASMA PROTEOMICS - LOOKING FOR NEW BIOMARKERS

.19

S6_A2

DISEASE SPECIFIC ALBUMIN PATTERNS DEFINED BY ELECTRON SPIN RESONANCE

S6_A3

PATTERN RECOGNITION BIOMATHEMATICS FOR BIOMARKER DEVELOPMENT

S7_A1

HER-2/NEU ONCOPROTEIN SERUM LEVELS IN PATIENTS WITH BREAST CANCER:

COMPARISON WITH CEA AND CA 15.3.

S7_A2

PUNCTA, BREAST CANCER AND HER2/NEU. PROTOTYPE OF A COMPUTER SUPPORT

FOR THE VISUALIZATION OF HEALTH CARE CONSUMPTION

S7_A3

CLINICAL VALUE OF SERUM HER-2/NEU TESTING

S7_A4

REASSESSMENT OF HER2 STATUS IN BREAST CANCER PATIENTS AT THE TIME

OF METASTATIC DISEASE BY SERUM HER2 AND HER2 STATUS OF CIRCULATING

TUMOR CELLS.

S8_A1

THE ORIGIN OF CANCER DNA METHYLATION PROFILES

S8_A2

EPIGENETIC SILENCING OF GENES INVOLVED IN SIGNALING PATHWAYS

IN GASTRIC CANCER

S9_A1

ANALYTICAL AND CLINICAL FACTORS IN THE QUALITY AND STANDARDIZATION OF TWO

PROSTATE CANCER TESTS

S9_A2

HER2 GUIDELINE DEVELOPMENT TO IMPROVE TESTING QUALITY.... 
S9_A3

ASSESSING QUALITY - AN EQA PERSPECTIVE

S9_A4

QUALITY REQUIREMENTS IN CLINICAL PRACTICE - A USER'S VIEW

S10_A1

PEPTIDE TRUNCATION BY DIPEPTIDYL PEPTIDASES : IMPORTANCE IN PHYSIOLOGICAL

AND PATHOLOGICAL PROCESSES

S10_A2

DIPEPTIDYL PEPTIDASE IV (DPP-4) INHIBITION AS A TREATMENT FOR TYPE 2 DIABETES .......26

S10_A3

DIPEPTIDYL PEPTIDASE IV AND RELATED MOLECULES: MARKERS

AND GROWTH REGULATORS OF HUMAN GLIOMAS.

S10_A4

SEPRASE IN THE PRE-METASTATIC NICHE AND TUMOR INTRAVASATION .27

S10_A5

CLUSTERING AND PROTEOLYTIC ENZYMES COMPLEXES FORMATION IN SPECIALIZED

PLASMA MEMBRANE DOMAINS OF ENDOTHELIAL CELLS. ROLE IN ANGIOGENESIS. .28

S10_A6

NOVEL TARGETED THERAPIES IN LUNG CANCER

S11_A1

IN VIVO IMAGING AND IN VITRO TUMORMARKER DETERMINATION IN CANCER

OF THE THYROID, BREAST, AND SKIN

S11_A2

PET, CT AND TUMOR MARKERS IN LUNG CANCER

S11_A3

PET/CT AND PSA IN PROSTATE CANCER.

S11_A4

PET/CT AND TUMOR MARKERS IN GYNECOLOGICAL CANCER 30

S11_A5

FDG AND CC-PET AS A MODEL FOR TRACER AND DRUG MICRODOSING .

31

S12_A1

DENDRITIC CELL-BASED VACCINES FOR CANCER: THE CURRENT STATUS AND FUTURE

APPROACHES

S12_A2

MOLECULARLY DEFINED VACCINES FOR IMMUNOTHERAPY OF CANCER

S12_A3

IMMUNOSTIMULATORY/IMMUNOPROTECTIVE EFFECTS OF MACROMOLECULAR

ANTICANCER THERAPEUTICS.

S13_A1

LC-MS/MS PROCEDURE FOR MEASURING ZN-ALPHA2-GLYCOPROTEIN AS A TUMOR

MARKER FOR PROSTATE CANCER

S13_A2

MASS ANALYSIS TO DESCRIBE POST-TRANSLATIONAL CHANGES IN PROTEINS: LESSONS

LEARNED FROM METABOLIC DISEASES

S13_A3

GENOMIC REARRANGEMENTS IN THYROID CANCER AND THE POTENTIAL

ORGAN-STEM CELL ORIGIN OF THYROID NEOPLASIA.

S13_A4

CIRCULATING ENDOTHELIAL CELLS AND PRECURSOR CELLS AS PREDICTORS OF CANCER SPREAD AND METASTASIS: PILOT DATA ON COLORECTAL AND BREAST CANCER ................35

S13_A5

OUTLINING PHARMACOGENETIC APPROACHES IN CANCER TREATMENT .36 
S14_A1

COMPUTER SUPPORTED RETRIEVAL OF INFORMATION NEEDS AND INFORMATION

CONTENTS

S14_A2

TUMOR MARKERS IN CERVICAL CANCER FOCUSED ON THE USE OF SCC

S14_A3

GUIDELINES FOR THE USE OF BIOMARKERS IN BREAST CANCER

38

S14_A4

A CRITIQUE OF THE AMERICAN(NACB) AND EUROPEAN (EGTM) GUIDELINES FOR

THE USE OF TUMOR MARKERS IN DETECTION AND MONITORING CANCER .38

S15_A1

ANTIBODY-CYTOKINE COMPLEXES: MAGIC TOOLS FOR TUMOR IMMUNOTHERAPY?

S15_A2

TRANSCRIPTIONAL REGULATION IN MELANOMA PROGRESSION: IMPLICATIONS FOR

THERAPY

S15_A3

THE ROLE OF THE IL-1 MOLECULES IN CARCIONGENSIS, TUMOR INVASIVENESS

AND TUMOR-HOST INTERACTIONS

S15_A4

CHEMOPREVENTION OF PROSTATE CANCER WITH VITAMIN D .40

S16_A1

REGULATION OF MTS GENE EXPRESSION

S16_A2

INTERPRETATION OF NATURAL FLUCTUATIONS OF METALLOTHIONEINS AND METAL

LEVELS IN THE AQUATIC ORGANISMS

.42

S16_A3

COUPLING TECHNIQUES FOR METALLOTHIONEIN ANALYSIS AND CHARACTERIZATION .....43

S16_A4

METALLOTHIONEINS AS A NEW POTENTIAL TUMOR MARKER.

S16_A5

SERUM LEVELS OF METALLOTHIONEIN IN EXPANSIVE CONTUSIONS, INTERLEUKINE-6, AND TNF-DURING REPARATIVE PHASE OF THE BLOOD BRAIN BARRIER DAMAGE .44

S17_A1

FECAL OCCULT BLOOD TEST (FOBT) - COMMON GUAIAC VERSUS IMMUNOLOGICAL TEST..45 S17_A2

VIRTUAL VERSUS REAL COLONOSCOPY .45

S17_A3

MOLECULAR DNA DIAGNOSIS OF CRC 46

S17_A4

DNA METHYLATION IN CRC

.46

S17_A5

PROTEOMICS IN CRC

S17_A6

PLASMA TIMP-1 COMBINED WITH PLASMA CEA MEASUREMENTS IN RISK ASSESSMENT

FOR CRC. A POPULATION BASED, PROSPECTIVE STUDY OF 5,005 INDIVIDUALS AT RISK

FOR CRC.

S17_A7

MULTIPARAMETRIC ANALYSIS IN CRC .48

S18_A1

EUROPEAN RANDOMIZED STUDY (ERSPC): DOES SCREENING DETECT TOO MANY

PROSTATE CANCERS? 
S18_A2

HOW DOES PSA TESTING IMPACT QUALITY OF LIFE?

S18_A3

PCA3: A MOLECULAR URINE PROSTATE CANCER TEST . .50

S18_A4

DNA METHYLATION MARKERS FOR DIAGNOSIS AND PROGNOSIS OF PROSTATE CANCER.....51

S18_A5

P-MARK: A EUROPEAN QUEST FOR NEW DIAGNOSTIC AND PROGNOSTIC MARKERS FOR

PROSTATE CANCER

S18_A6

EFFECTS OF PSA ASSAY STANDARDIZATION ON CLINICAL DECISION MAKING 52

S19_A1

DISSECTING CELL FATE REGULATION IN STEM CELLS 53

S19_A2

GENETIC SIGNATURES OF "STEMNESS" PATHWAYS IN THERAPY-RESISTANT HUMAN

CANCERS AND THEIR THERAPEUTIC IMPLICATIONS 53

S19_A3

BREAST CANCER STEM CELLS: BIOLOGICAL AND CLINICAL IMPLICATIONS .54

S20_A1

THYMIDINE KINASE 1 AS MARKERS FOR CELL PROLIFERATION IN MALIGNANCIES;

DESIGNED PEPTIDE ANTIBODIES USED FOR HISTOCHEMISTRY AND SERUM TK STUDIES....55

S20_A2

ULTRA SENSITIVE COLOROMETRIC THYMIDINE KINASE ACTIVITY ANALYSES USING

TEMPLATE DEPENDENT PRODUCT IMMOBILISATION BY REVERSE TRANSCRIPTASE

COMPLEMENTED BY YEAST KINASES

S20_A3

DEVELOPMENT OF AN AUTOMATED, NON-RADIOMETRIC ASSAY FOR THYMIDINE KINASE

ON THE LIAISON ${ }^{\circledR}$ ANALYZER - TECHNICAL AND CLINICAL EVALUATION

S20_A4

THYMIDINE KINASE ENZYMATIC ACTIVITY - GOOD MARKER FOR THE EARLY

RECOGNITION OF THE RELAPSE OF ACUTE LEUKEMIA IN CHILDREN.

S20_A5

THE CLINICAL VALUE OF THYMIDINE KINASE IN HEMATOLOGICAL MALIGNANCES .57

S20_A6

THE DIAGNOSTIC AND PROGNOSTIC SIGNIFICANCE OF THYMIDINE KINASE (TK)

IN TUMOR DISEASES

S20_A7

CLINICAL UTILITY OF THYIMIDINE KINASE (TK) IN LYMPHOPROLIFERATIVE

MALIGNANCIES

S21_A1

CLINICAL USE OF CIRCULATING TUMOR CELLS IN THE MANAGEMENT OF PATIENTS

WITH BREAST CANCER

S21_A2

CLINICAL RELEVANCE OF THE MOLECULAR ANALYSIS OF CIRCULATING TUMOR CELLS

(CTC) .59

S22 A1

ARTIFICIAL NEURAL NETWORKS AND NOMOGRAMS IN PROSTATE CANCER

S22_A2

THE PROSTATE CANCER PREVENTION TRIAL PROSTATE CANCER RISK CALCULATOR 60

S22_A3

STATISTICAL PREDICTION MODELS AND MEDICAL DECISION MAKING 
S22_A4

THE ROC CURVE AND THE BRIER SCORE FOR EVALUATING SINGLE TUMOR MARKERS

AND COMPLEX RISK PREDICTION MODELS .....................................................61

S22_A5

MULTIPLE MARKER EVALUATION FOR PANCREATIC CANCER DIAGNOSIS

S22_A6

PREDICTIVE AND PROGNOSTIC SERUM MARKERS IN ADVANCED LUNG CANCER

S22_A7

PATTERN OF RELEASE OF VARIOUS ONCOLOGICAL BIOMARKERS IMPROVES

THE DIAGNOSTIC CAPACITY OF LUNG CANCER

WS1

NEW ANTIBODIES TO SCCA: SECOND TD-10 REPORT

FC1_A1

A NOVEL MULTIPLE BIOMARKER ASSAY FOR THE DETECTION OF OVARIAN CARCINOMA

FC1_A2

PROPERTIES OF MELANOSOMES AND THEIR EXPLOITATION IN MELANOMA DIAGNOSIS

AND TREATMENT

FC1_A3

SELECTIVE SUPPRESSION OF HUMAN PROSTATE TUMOR GROWTH IN MICE BYA

RECOMBINANT ANTI-PSMA IMMUNOTOXIN

FC1_A4

AUTOANTIBODIES TO ANNEXINS AND CYTOKERATINS

FC2_A1

EARLY DETECTION OF METASTATIC BREAST CANCER DISEASE BY USING

TUMORMARKERS

.70

FC2_A2

AFAMIN A NOVEL BIOMARKER FOR THE DIAGNOSIS OF PRIMARY TESTICULAR GERM

CELL CANCER - PRIMARY RESULTS.

FC2_A3

AFAMIN AND APOLIPOPROTEIN A-IV - NOVEL PROTEIN MARKERS FOR OVARIAN

CANCER

FC2_A4

PET IMAGING WITH CU-64 LABELED HUMANIZED ANTI-CEA ANTIBODY

FC2_A5

ANALYSES OF COMPARATIVE GENOMIC HYBRIDIZATION (CGH) ARRAYS ON URINARY

SPECIMENS OF PATIENTS WITH BLADDER CANCER

FC2_A6

FREQUENT EPIGENETIC INACTIVATION OF DICKKOPF FAMILY GENES IN

GASTROINTESTINAL TUMORS

FC2_A7

LUNG CANCER - CAN AUTOANTIBODIES PROVIDE AN AID TO DIAGNOSIS? 73

FC2_A8

IMMUNOTHERAPY PROLONGS THE SERUM CEA-TPA-CA15.3 LEAD TIME

AT THE METASTATIC PROGRESSION IN ENDOCRINE-DEPENDENT BREAST CANCER

PATIENTS.

FC2_A9

CYFRA 21-1 AND CEA AS PROGNOSTIC FACTORS IN OPERATED STAGE I NON SMALL CELL LUNG CANCER PATIENTS?

P1_1

CELL CYCLE DISTURBANCES AND MITOTIC CATASTROPHES IN CELL LINES FOLLOWING

LOW DOSE RATE BETA-IRRADIATION. 
P1_2

ANDROGEN INHIBITS THYMOSIN BETA4 EXPRESSION IN PROSTATE CANCER CELLS...........76

P1_3

TNF EFFECTS ON HEMATOLOGICAL CELL LINES .............................................. 77

P1_4

FREE AND N-(2-HYDROXYPROPYL)METHACRYLAMIDE (HPMA) BOUND DOXORUBICIN:

DIFFERENCES IN GLYCOSYLATION OF EL-4 CELL SURFACE MEMBRANE

P1_5

DIFFERENCES IN REACTIVITIES TO SCCA1 AND SCCA2 MEASURED WITH ARCHITECT SCC AND IMX SCC

P1_6

GENOMIC SCREENING FOR GENES SILENCED BY DNA METHYLATION IN MULTIPLE

MYELOMA

P2_1

IGF-I RECEPTOR IS A CANDIDATE MOLECULAR TARGET FOR BOTH HUMAN ESOPHAGEAL

SQUAMOUS CELL CARCINOMA AND ADENOCARCINOMA ..........................................79

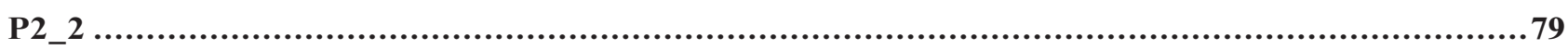

NEW PERSPECTIVES IN ELECTROCHEMICAL DETERMINATION OF METALLOTHIONEINS.....79

P2_3

URINARY EXCTRETION OF THE TRASFORMING GROWTH FACTOR- $\alpha$ (TGF- $\alpha$ ) AND THE

EPIDERMAL GROWTH FACTOR(EGF)OF THE PATIENTS WITH BENIGN OR MALIGNANT

DISEASES: APPRAISAL ON CANCEROGENESIS.

P2_4

METHYLATION STATUS OF THE p16 TUMOR-SUPPRESSOR, DAP-kinase AND APC TUMOR-

SUPPRESSOR GENES IN COLON BIOPSIES

P2_5

CLEAVABILITY OF HPMA-BASED CONJUGATES BEARING DOXORUBICIN BOUND

BY AMIDIC BOND IS NOT A PREREQUISITE OF THEIR EFFICACY .

P2_6

SOMATIC HYPERMUTATION IN THE REARRANGED VH BUT NOT IN THE V-LAMBDA

GENES IN EBV TRANSFORMED RHEUMATOID FACTOR-PRODUCING LYMPHOBLASTOID

CELL LINE.

P2 7

THE POSITIVE TRANSCRIPTION ELONGATION FACTOR B MEDIATES MYOCYTE

ENHANCER FACTOR 2-DEPENDENT TRANSCRIPTION

P2_8

ALTERATIONS OF DIHYDROPYRIMIDINE DEHYDROGENASE (DPD) AND PYRIMIDINASE

(DPYS) ARE NOT PREDICIVE MARKERS OF TOXICITY IN ONCOLOGY PATIENTS TREATED

BY FLUOROPYRIMIDINES

P2_9

QUANTITATIVE ELISA STUDY OF MMPs AND TIMPs EXPRESSION PATTERN

IN THE TUMORS AND PLASMA OF COLORECTAL CANCER PATIENTS .83

P3_1

ANTI-EGFR TARGETED POLYMERIC CONJUGATES AND MECHANISM OF THEIR

INTERNALIZATION

P3_2

INVESTIGATION OF THE HUMORAL IMMUNE RESPONSE TO THE TUMOR ASSOCIATED

ANTIGEN PSA IN BREAST CANCER

P3_3

TUMOR SUPPRESSION BY APOPTOSIS INDUCED WITH ANTI-ERBB-2 CHIMERIC

MONOCLONAL ANTIBOBY .85 
P3_4

IMMUNOCOMPLEXES OF IL-2 AND ANTI-IL-2 MAB AS NOVEL PROMISING TOOL

FOR CANCER IMMUNOTHERAPY OR COMBINED CHEMO/IMMUNOTHERAPY ....

P3_5

EFFECT OF ANTI-ANGIOGENIC THERAPY ON CYTOKINE PROFILE IN PATIENTS WITH

METASTATIC COLORECTAL CANCER

P3_6

HPMA-BASED MACROMOLECULAR THERAPEUTICS REINFORCE ANTI-TUMOR

IMMUNITY... .86

P3_7

$\alpha_{M} \bar{\beta}_{2}$ INTEGRIN MODULATOR INHIBITS LYMPHOMA GROWTH IN VIVO AND REDUCES

SQUAMOUS CELL CARCINOMA TUMOR INVASION BY BLOCKING LEUKOCYTE

INFILTRATION

P3_8

TREATMENT OF EXPANDING TUMORS BY TARGETED HPMA COPOLYMER-BOUND

DOXORUBICIN CONJUGATES IS FOLLOWED BY SPECIFIC ANTI-TUMOR IMMUNITY

P3_9

POLYMERIC MICELLES WITH DOXORUBICIN BOUND THROUGH HYDRAZON BOND -

A NEW PROMISING ANTITUMOR DRUG DELIVERY SYSTEM. .88

P4_1

UTILITY OF MELANOMA INHIBITORY ACTIVITY (MIA) IN MALIGNANT MELANOMA

PATIENTS, COMPARISON WITH S100B

P4_2

EXPLORATION OF CEA EXPECTED VALUES AND CUT-OFFS IN HEALTHY

AND COLORECTAL CANCER PATIENTS USING VIDAS ${ }^{\circledR}$ CEA(S) ASSAY

P4_3

OVARY CANCER ASCITES PROTEINS - 1: CHARACTERIZATON OF CA125 MONOCLONAL

ANTIBODY (OC125) BINDING PROTEINS .90

P4 4

PET IMAGING OF PROSTATE CANCER WITH A HIGHLY SPECIFIC ANTI-PSMA-ANTIBODY . .90

P4_5

A PANEL OF TUMOR ANTIGENS AND AUTOANTIBODIES FOR THE DETECTION OF LUNG

CANCER

P4_6

COMPARISON OF CATCHER ANTIBODIES IN MANUAL TPSTM ASSAY 91

P4_7

CHEMILUMINESCENT ASSAY (CIA) FOR THE RECEPTOR OF ALPHA FETOPROTEIN (RECAF) TO SEPARATE CANCER FROM NORMAL SERA

P4_8

EVALUATION OF PLASMA AND SERUM COLLECTION TUBES IN THE RECEPTOR

FOR ALPHA FETOPROTEIN (RECAF) RADIOIMMUNOASSAY (RIA)

P4_9

SHORT- AND LONG-TERM STABILITY OF TIMP-1 IN PLASMA SAMPLES.

P4_10

STNF RI IS AN INDEPENDENT PROGNOSTIC FACTOR IN BREAST CANCER PATIENTS 93

P4_11

DIAGGNOSTIC CAPACITY OF TIMP-1 AS COMPARED TO CEA IN COLORECTAL CANCER .94

P4_12

SERUM HER2/NEU LEVELS IN BREAST CANCER PATIENTS WITH DIFFERENT

LEVEL OF TUMOR HER2/NEU PROTEIN EXPRESSION .95 
P4_13

EVALUATION OF A PANEL OF TUMOR MARKERS IN PLEURAL FLUID FOR THE DIFFERENTIAL

DIAGNOSIS OF BENIGN VERSUS MALIGNANT EFFUSIONS

P4_14 96

PROTEOMIC ANALYSES OF ESOPHAGEAL SQUAMOUS CELL CARCINOMA..........................96

P4_15

THYMIDINE KINASE AS A ADITIONAL MARKER IN SOLID TUMORS

.96

P4_16

ESTABLISHMENT OF MONOCLONAL ANTIBODIES AGAINST ONCOPROTEIN E7

OF HIGH-RISK HUMAN PAPILLOMAVIRUSES FOR USE IN LIQUID-BASED CYTOLOGY

AND DEVELOPMENT OF ELISA ASSAYS

P4_17

ALTERATION IN MUC1 EXPRESSION IN BRONCHIAL EPITHELIUM IS AN EARLY MARKER

FOR RECURRENT AND METACHRONOUS BRONCHIAL CARCINOMA

P4_18

TUMOR MARKERS AND CYTOKINES IN PATIENTS WITH CERVICAL CANCER ......................98

P4_19

PROGRP IN DIFFERENTIAL DIAGNOSTICS OF SCLC AND NSCLC PATIENTS .98

P4_20

VEGF - A PROGNOSTIC FACTOR IN SMALL CELL LUNG CANCER PATIENTS? .99

P4_21

ANALYSIS OF FREQUENCY OF AMINO ACID SUBSTITUTIONS IN DIRECT AFP-LIKE

MOTIFS OF THE PROTEINS ASSOCIATED WITH EMBRYO- AND TUMORIGENESIS .99

P4_22

DIFFERENT FORMS OF SQUAMOUS CELL CARCINOMA ANTIGEN ( TOTAL SCCA, TOTAL SCCA1 AND TOTAL SCCA2) IN PATIENTS WITH BENIGN AND MALIGNANT DISEASES 100

P4_23

EVALUATION OF A NEW TUMOR MARKER, MESOMARK IN PATIENTS WITH BENIGN AND MALIGNANT DISEASES .

P4_24

POINT-OF-CARE SERUM TEST FOR CANCER DETECTION BASED ON THE RECAF CANCER

MARKER

P4_25

DIAGGOSTIC AND PROGNOSTIC VALUE OF SOLUBLE MESOTHELIN RELATED PROTEINS

(SMRP) IN SERA OF PATIENTS WITH MALIGNANT PLEURAL MESOTHELIOMA

P4_26

ESTABLISHMENT OF ENZYME IMMUNOASSAY FOR DETERMINATION OF HE4

102

P4_27

PREDICTIVE AND PROGNOSTIC ROLE OF SERUM CYFRA 21-1 IN ADVANCED NONSMALL

CELL LUNG CANCER: ASSOCIATION OF MARKER CHANGES AFTER TWO CYCLES OF CHEMOTHERAPY WITH CLINICAL RESPONSE AND SURVIVAL

P4_28

A NEW METHOD FOR DETERMINING SEROLOGICAL FORMS OF PRO GASTRIN-RELEASING

PEPTIDE

P4_29

PRODUCTION OF NEW MONOCLONAL ANTIBODIES TO THE ENTIRE proGRP(1-125)

PEPTIDE.

P4_30

MONOTOTAL - NEW MARKER FOR NSCLC. 
P4_31

OVARY CANCER ASCITES PROTEINS - 2: IDENTIFICATION OF POLYPEPTIDE NOT BINDING

MONOCLONAL ANTIBODY (OC125) TO CA 125

P4_32

CLINICAL VALUE OF S100 LEVELS IN SERUM OF MALIGNANT MELANOMA PATIENTS 105

P4_33

DES GAMMA-CARBOXY PROTHROMBIN PROMOTES PROGRESSION

OF HEPATOCELLULAR CARCINOMA

105

P4 34

RELEASE OF ONCOLOGIC BIOMARKERS IN PATIENTS WITH PROSTATE CANCER 106

P4_35

DETERMINATION OF APOPTOSIS INDUCED PEPTIDE IN THE HUMAN

ALPHA-FETOPROTEIN STRUCTURE

P4_36.

SHORT AFP-LIKE MOTIFS WITHIN EGF-LIKE MODULES OF THE PROTEINS REGULATING

EMBRYO- AND TUMORIGENESIS AS SIGNALS FOR CELL PROLIFERATION,

DIFFERENTIATION AND SURVIVAL

P4_37

ADULT HUMAN HEPATOCYTES EXPRESS ALPHA-FETOPROTEIN RECEPTOR WITHOUT

RELATION TO THE EXISTENCE OF HEPATITIS, LIVER CIRRHOSIS, OR HEPATOCELLULAR

CARCINOMA.

P4_38

A MULTI-ANALYTE BIOMARKER PANEL AND ALGORITHM PREDICT THE RISK

OF CANCER IN WOMEN WITH PELVIC MASS.

P4 39

HE4 - A NOVEL BIOMARKER FOR OVARIAN CANCER

108

P4_40

CHROMOGRANIN A

109

P4_41

STABILITY OF PRO-GASTRIN RELEASING PEPTIDE IN SERUM AND PLASMA

P4_42

RECOVERY OF THE WHO FIRST INTERNATIONAL STANDARD 72/225 FOR AFP

BY THE ABBOTT ARCHITECT AFP ASSAY

P4_43

TUMOR MARKER KINETICS (TMK) OF CEA AND CA15-3 CORRELATE WITH RESPONSE

IN PATIENTS UNDERGOING CHEMOTHERAPY FOR METASTATIC BREAST CANCER.

P5_1

IMMUNOMAGNETIC BEAD ENRICHMENT OF CIRCULATING TUMOR CELLS - RECOVERY

EXPERIMENTS

P5_2

DETECTION OF P15 GENE PROMOTER METHYLATION IN SERUM IN GASTRIC CANCER

PATIENTS

P5_3

TRAKING-LINE USE: EXPERIENCE IN A LABORATORY ANALYSIS WITH A WIDE CANCER ASSAY PANEL

P5_4

AFP ASSAY ON OLYMPUS AU3000I IMMUNOASSAY ANALYSER

P5_5

BIOLOGICAL AND ANALYTICAL VARIATIONS OF CA 15.3 ASSAYS : INFLUENCE

ON CUTOFFS AND REFERENCE CHANGE VALUE 
P5_6

REFERENCE CHANGE VALUES AND DISEASE SPECIFIC CUTOFF FOR CA 125 ASSAYS 113

P5_7

TOTAL PSA WHO CALIBRATION : IMPACT ON DECISION POINT. 114

P5_8

PERFORMANCE EVALUATION OF AN AUTOMATED IMMUNOASSAY FOR

THE DETERMINATION OF AFP (Alpha-fetoprotein) ON THE OLYMPUS AU3000i ${ }^{\mathrm{TM}}$

IMMUNOASSAY SYSTEM. 114

P5_9

PERFORMANCE EVALUATION OF AN AUTOMATED IMMUNOASSAY FOR

THE DETERMINATION OF BR-TC [BR-Tumor Check(CA 153 antigen)] ON THE OLYMPUS

AU3000i'M IMMUNOASSAY SYSTEM.

P5_10

PERFORMANCE EVALUATION OF AN AUTOMATED IMMUNOASSAY FOR THE

DETERMINATION OF CEA (Carcinoembryonic Antigen) ON THE OLYMPUS AU3000i ${ }^{\mathrm{TM}}$

IMMUNOASSAY SYSTEM

P5_11

PERFORMANCE EVALUATION OF AN AUTOMATED IMMUNOASSAY FOR THE

DETERMINATION OF GI-TC [GI-TUMOR CHECK(CA 19-9 antigen)] ON THE OLYMPUS

AU3000i' ${ }^{\mathrm{TM}}$ IMMUNOASSAY SYSTEM

P5_12

PERFORMANCE EVALUATION OF AN AUTOMATED IMMUNOASSAY FOR

THE DETERMINATION OF TOTAL PSA (Total Prostate Specific Antigen) ON THE OLYMPUS

AU3000; ${ }^{\mathrm{TM}}$ IMMUNOASSAY SYSTEM

P5_13

THE CLINICAL UTILITY OF ARCHITECT ${ }_{\mathrm{XR}}$, IMMUNO-1, AND ELECSYS ASSAYS

FOR CA 19-9

P6_1

CLINICAL IMPACT OF USE OF DIFFERENT PSA ASSAYS IN PROSTATE CANCER SCREENING.

P6_2

RESULTS OF FOLLOW UP USING TUMOR MARKERS AND PET/CT IN COLORECTAL

CANCER

P6_3

PSA-VELOCITY AND ARTIFICIAL NEURAL NETWORK (ANN)-VELOCITY

TO DIFFERENTIATE PROSTATE CANCER FROM BENIGN PROSTATIC DISEASE - RESULTS

FROM 10 YEARS OF ROUTINE FREE PSA USE

P6_4

PHARMACOKINETIC TRANSLATIONAL STUDY IN ADVANCED SOLID TUMORS OF 5-FU

WITH AN ADMIXTURE OF A GALACTOMANNAN DERIVATIVE

P6_5

PONS-S TRIAL (PATIENT ORIENTED SURVEILLANCE-STUDY): INTENSIFIED

FOLLOW-UP OF BREAST CANCER PATIENTS BASED ON TUMOR MARKER KINETICS

AND HIGH-END IMAGING

P7_1

BIOCHEMICAL STUDY OF THE BREAST CYSTIC FLUID AND CANCER RISK

P7_2

INFLUENCE OF HHV-6 AND HHV-7 ACTIVATION ON IMMUNOLOGICAL PARAMETERS

IN COLORECTAL CANCER PATIENTS

P7_3

ATTRACTIN AND DP4 ACTIVITY 
P7_4

DIPEPTIDYL PEPTIDASE 4 AND FIBROBLAST ACTIVATION PROTEIN ALPHA AS TUMOR SUPPRESSOR GENES IN SOFT TISSUE SARCOMAS.

122 P7_5

STUDY OF BINDING OF PLATINUM BASED CYTOSTATICS TO DNA STRUCTURE;

INFLUENCE OF GLUTATHIONE

123

P7_6

PARAMAGNETIC BEADS COUPLED WITH ELECTROCHEMICAL DETECTION

AS A TOOL TO INVESTIGATE TRANSCRIPTOME

124

P7_7

SONODYNAMIC THERAPY OF CANCER USING A NOVEL PORPHYRIN DERIVATIVE, DCPH-P-NA(I), WHICH IS DEVOID OF PHOTOSENSITIVITY.

124 P7_8

THE ROLE OF KRÜPPEL-LIKE FACTOR 6 (KLF6) IN DIFFERENTIATION

OF EMBRYONIC STEM CELLS INTO HEPATOCYTES

125

P7_9

SEARCHING URINARY TUMOR MARKERS FOR BLADDER CANCER USING A TWO

DIMENSIONAL DIFFERENTIAL GEL ELECTROPHORESIS (2D-DIGE) APPROACH ...

125

P7_10

GALECTIN-3 EXPRESSION IS ASSOCIATED WITH BLADDER CANCER PROGRESSION

AND CLINICAL OUTCOME.

126

138 


\section{AUTHOR INDEX}

\begin{tabular}{|c|c|}
\hline Abubaker Y. & 119 \\
\hline Adachi Y. & 79 \\
\hline Adam V. & $43,44,79,123,124$ \\
\hline Albert W. H. & 59 \\
\hline Alexandrova I.A. & 106 \\
\hline Alfthan $\mathrm{H}$. & 117 \\
\hline Algaba F. & 125 \\
\hline Allaeys J.M. & 114 \\
\hline Allard W. J & $67,108,108$ \\
\hline Alt K & 68,90 \\
\hline Ankerst D.P. & 60,48 \\
\hline Anselmi L & 74 \\
\hline Apte R. N. & 40 \\
\hline Aragona P. & 80,121 \\
\hline Arndt B. & 118 \\
\hline Arnold G. J. & 47 \\
\hline Aronsson A.Ch. & $56,57,58$ \\
\hline Asano R. & 124 \\
\hline Atchison M. L. & 10 \\
\hline Auge J.M. & $12,89,21,100,100$ \\
\hline Azvolinsky A. & 10 \\
\hline Babaian R. & 7 \\
\hline Bading J. & 72 \\
\hline Bangma Ch. H. & 49,51 \\
\hline Bär J. & 122 \\
\hline Barak V. & $11,58,102$ \\
\hline Barnes AC; & 73 \\
\hline Barnhill S. D. & 20 \\
\hline Baron-Wunderle V. & 89 \\
\hline Basko J. & 96 \\
\hline Bast R. & 67,108 \\
\hline Bauerfeind I & 11,70 \\
\hline Bauernfeind I. & 110 \\
\hline Baum R.P. & 120 \\
\hline Bednarikova M., & 36 \\
\hline Beklova M. & 123 \\
\hline Bellati P. & 112 \\
\hline Ben -Yehuda D. & 58 \\
\hline Bengt $\mathrm{T}$. & 25 \\
\hline Bengtsson Ch. & 91 \\
\hline Bergen H. R. & 34 \\
\hline Berger P & 15,14 \\
\hline Betka J. & 84 \\
\hline Beyer H L. & 108 \\
\hline Bidart J.M. & 14,15 \\
\hline Bidzinski M. & 98 \\
\hline Bílek M. & 83 \\
\hline Binkova H. & 43 \\
\hline Biran H. & 102 \\
\hline Birken S. & 15,14 \\
\hline Blahova P. & 79 \\
\hline Blankenburg F. & 63,74 \\
\hline Blankenstein M.A. & 47 \\
\hline Bogdanovic G. & 77 \\
\hline Boka V. & 111 \\
\hline Bonfrer J. & 37 \\
\hline Borisenko S. & 90,104 \\
\hline Borovanský J & 68 \\
\hline Boucek J. & 84 \\
\hline Bouchelouche K. & 30,31 \\
\hline
\end{tabular}

\begin{tabular}{|c|c|}
\hline Bowman A. & 13 \\
\hline Boyman O. & 39 \\
\hline Braun L. & 37 \\
\hline Brezicka T. & 95 \\
\hline Bristow A. & 14 \\
\hline Brown A K. & 67,108 \\
\hline Brünner N & 47 \\
\hline Břízová H. & 9 \\
\hline Buda C. & 121 \\
\hline Buda F. & 80,121 \\
\hline Bühler P & 68,90 \\
\hline Büker N & 119 \\
\hline Bumbasirevic V & 77 \\
\hline Burges A. & 13,71 \\
\hline Burlandi M. & 80 \\
\hline Busek P. & 26 \\
\hline Cabinakova M. & 111 \\
\hline Cammann H. & 119 \\
\hline Cammareri F. & 80 \\
\hline Camous C. & 117 \\
\hline Campr V. & 9 \\
\hline Canesin G. & 126 \\
\hline Carbone D. P. & 79 \\
\hline Carpi A & 74 \\
\hline Casal I. & 125 \\
\hline Casals G. & 100 \\
\hline Casova M. & $12,57,104$ \\
\hline Cerna Z. & 56 \\
\hline Cerri A. & 112 \\
\hline Cigudosa G.J. C. & 72 \\
\hline Colcher D. & 72 \\
\hline Collareta M & 74 \\
\hline Colpitts T. & 91 \\
\hline Cooney D. & $114,115,115,116,117$ \\
\hline Cordón-Cardo C. & 126 \\
\hline Cortón M. & 125 \\
\hline Coy J. F. & 17,18 \\
\hline Cozma D. & 10 \\
\hline Čásová M. & 109 \\
\hline D'Amico Y. & 91 \\
\hline Dahlén U. & 102,103 \\
\hline Dal Canto E & 74 \\
\hline Davidsson I. & 91 \\
\hline Davis G & $47,93,94$ \\
\hline Daxenbichler G. & 71 \\
\hline Dbaly V & 26 \\
\hline de Bruijn HWA & 13 \\
\hline De Meester I. & 26 \\
\hline de Rochebouët B. Grimaudet & 114,116 \\
\hline Demlova R. & 36 \\
\hline Demuth H.U. & 122,122 \\
\hline Dienemann $\mathrm{H}$. & 101 \\
\hline Dieplinger B. & 70,71 \\
\hline Dieplinger $\mathrm{H}$. & 70,71 \\
\hline Dieplinger J. & 70 \\
\hline Dietl J. & 18 \\
\hline DiSilvestro P. & 108 \\
\hline Domingo J. & 89 \\
\hline Dong H. & 27 \\
\hline Donina S. & $96,111,121$ \\
\hline
\end{tabular}




\begin{tabular}{|c|c|c|c|}
\hline Donkers J. & 21 & Geschwindt R D. & 108 \\
\hline Dontu G. & 54 & Ghersi G. & 28 \\
\hline Doss R & $47,93,94$ & Gil Y & 81 \\
\hline Dowell B & $47,92,92,93,94,109$ & Ginestier Ch. & 54 \\
\hline Dresse M. & 93 & Giunta M. L. & 28 \\
\hline Du Yao-Wu & 96 & Glinsky G. V. & 53 \\
\hline Dubska L. & 35 & Glover C L. & 108 \\
\hline Duffy M J & 3,38 & Goike H. & 91,13 \\
\hline Duke-Cohan J. S. & 3 & Goldmann-Posch U. & 120 \\
\hline Eckschlager T. & 43,123 & Gonzalez-Peramato P. & 72 \\
\hline Eickmeier B. & 31 & Gonzalez-Peramato P. & 126 \\
\hline Einarsson R. & 13 & González-Peramato P., & 125 \\
\hline Elsässer-Beile U & 68,90 & Granai C.O. & 67,108 \\
\hline Engele L. & 121 & Grande S. & 10 \\
\hline Erikson J. & 10 & Gras J. & 113,113 \\
\hline Eriksson D. & 76 & Graze I. & 70,71 \\
\hline Eriksson S. & 55 & Grebe S. K. G. & 35 \\
\hline Ermilova V. & 95 & Gregorin J. & 118 \\
\hline Ertl I. & 70 & Greither T. & 122 \\
\hline Essink-Bot M.L. & 50 & Gronowitz J.S. & 55 \\
\hline Esteller M. & 6 & Gruber Ch. & 63 \\
\hline Etrych T. & $33,77,81,85,86,88$ & Gurachevsky A. & 19 \\
\hline Fabrik I. & 43,79 & Haas O.A. & 8 \\
\hline Farrus B. & 21 & Haglund C. & 117 \\
\hline Favier J. & 13 & Hachimine $\mathrm{K}$. & 124 \\
\hline Fehm T. & 22 & Hall Ch. & 95 \\
\hline Feldmann K. & 62 & Hallensleben E. & 13 \\
\hline Fenske J. & 56 & Haltmayer M. & 70 \\
\hline Fermér Ch. & 97 & Hammond E & 24 \\
\hline Ferrari P & 74 & Harlozinska A. & 13 \\
\hline Ferrero A. & 13 & Harris R. & 112 \\
\hline Fidlerová J. & 82 & Hatz R. & 63,74 \\
\hline Filella X. & $12,21,89,100,100$ & Hauch S. & 59 \\
\hline Fillée C. & $113,113,114$ & Havel L. & 124 \\
\hline Filonenko E.V. & 97 & Hayashi T. & 78 \\
\hline Finek J. & 30,57 & He Q. & 55 \\
\hline Fink D. & 13 & He Qing-Yu & 96 \\
\hline Fitzgerald D. & $114,115,115,116,117$ & Heching N. & 102 \\
\hline Fleisher M., & 38 & Heinemann V. & $11,48,62,70,110,120$ \\
\hline Foster B. A. & 40 & Heiser U. & 122 \\
\hline Frank G.A. & 97 & Hellstrom I & 108 \\
\hline Friedman M. & 58 & Hellstrom K.E. & 108 \\
\hline Friedman S.L. & 125 & Herth F.J.F. & 101 \\
\hline Friedrich D. & 122 & Hevér-Pálfy T. & 80 \\
\hline Fritsche H. & 7,59 & Hirano K. & 76 \\
\hline Fröhlich T. & 47 & Hirat H. & 85 \\
\hline Frost $\mathrm{S}$. & 92 & Hodawadekar S. & 10 \\
\hline Frost S. & 92 & Hoffmann H. & 101 \\
\hline Fujikawa T. & 105 & Hoffmann T. & 122,122 \\
\hline Fujinaga K & 82 & Holdenrieder S. & 62 \\
\hline Fujita K. & 109 & Holubec L. & 30 \\
\hline Fuksiewicz M. & 93,98 & Holubec L. Jr. & $56,104,109,57,12$ \\
\hline Fulker M. & 84 & Honig A. & 18 \\
\hline Fuloria J. & 119 & Horakova Z. & 43 \\
\hline Gaarenstroom K. & 37 & Horna A. & 123 \\
\hline Galamb B. & 80 & Hotakainen $\mathrm{K}$. & 117 \\
\hline Galamb O. & 80 & Houstava L. & 44 \\
\hline Ganser E.M. & 94 & Hovorka O. & $81,84,88$ \\
\hline Gaston S.J.S. & $114,115,115,116,117$ & Hrabeta J. & 43,123 \\
\hline Gat M. & 58 & Hrdinova V. & 79 \\
\hline Gerber A. & 92,92 & Hrubý M. & 88 \\
\hline Gerds T. A. & 61 & $\mathrm{Hu}$ X. & 101 \\
\hline Gershtein E. & 83,95 & Hubalek J. & 124 \\
\hline
\end{tabular}




\begin{tabular}{|c|c|c|c|}
\hline Huber A. & 118 & Kley K. & 29 \\
\hline Hubert A. & 11 & Klocker H. & 70 \\
\hline Huland $\mathrm{H}$. & 60 & Klyosov A.A. & 119 \\
\hline Huska D. & 123,124 & Kobayashi E. & 78 \\
\hline Chapenko S. & 121 & Kodet R. & 9 \\
\hline Chapman C. & $69,84,73$ & Kofler K. & 70 \\
\hline Chen D. & 27 & Kohrenhagen N. & 18 \\
\hline Chen W.T. & 27 & Koivunen E. & 87 \\
\hline Chenevoy N. & 89 & Kojic V & 77 \\
\hline Cherasse A. & 114,117 & Kolligs F.T. & 46 \\
\hline Chezar I & 81 & Korfage I. & 50 \\
\hline Chissov V.I. & 97 & Kormunda S. & $12,57,56,104,109$ \\
\hline Chiu Jen-Fu & 96 & Korzeniowski S. & 99 \\
\hline Chmelová H. & 85 & Kotaska K. & 44 \\
\hline Cholujová D. & 86 & Kotowicz B. & 93,98 \\
\hline Christensen IJ & 47 & Kováŕ L. & 77 \\
\hline Chun F. K.-H. & 60 & Kovář M. & $33,85,88$ \\
\hline Chytil P. & 77 & Kowalska M. & 93,98 \\
\hline Iguchi K. & 76 & Kozireva S. & 111 \\
\hline Ihor L. & 53 & Kraguljac N & 77 \\
\hline Ilelaboye O. & 115,115 & Krepela E & 26 \\
\hline Illert B. & 18 & Krieg C. & 39 \\
\hline Imai K & $82,23,73,79,85$ & Krizkova S. & $43,79,123,124$ \\
\hline Imsumran A. & 79 & Krockenberger $\mathrm{M}$. & 18 \\
\hline Inçaurgarat B. & 89 & Kronenberg F. & 70 \\
\hline Irato $\mathrm{P}$. & 42 & Krsková L. & 9 \\
\hline Ito $\mathrm{M}$. & 76 & Kukacka J. & $43,44,79,123$ \\
\hline Itoh F. & 125 & Kula K. & 110 \\
\hline Jakimov D & 77 & Kulpa J. & 99 \\
\hline Jakubíková J. & 86 & Kuroki M. & 124,124 \\
\hline Janatkova I. & 111 & Kushlinsky N. & 95 \\
\hline Janetschek G. & 70 & Kwiatkowski M. & 118 \\
\hline Jaraczewski J. & 110 & Laessig D. & 110 \\
\hline Jauffret E. & 54 & Laird P. W. & 23 \\
\hline Jaunalksne I. & 96,121 & Lambeir A.M. & 26 \\
\hline Johansson L. & 76 & Lamerz R. & 15 \\
\hline Jonska J. & 98 & Lankiewicz S. & 59 \\
\hline Jung K. & 119 & Larizza L. & 8 \\
\hline Jurisic V & 77 & Laskov R & 81 \\
\hline Kahlert S. & 110 & Lässig D. & 11,70 \\
\hline Kainz Ch. & 13 & Lasutina T.N. & 105 \\
\hline Kalickman I. & 11,58 & Lau-Werner U. & 48,94 \\
\hline Kalinová M. & 9 & Lee Ch.T. & 79 \\
\hline Kaminska J. & 93,98 & Lein M. & 119 \\
\hline Kämmerer U. & $17,18,18$ & Lentwojt E. & $114,115,115,116,117$ \\
\hline Karakiewicz P.I. & 60 & Lesche R. & 51 \\
\hline Karlsson M. & 97 & Letourneau S. & 39 \\
\hline Karmakova T.A. & 97 & Letyagin V. & 95 \\
\hline Kato Y. & 79,85 & Levy M. & 118 \\
\hline Kattan M.W. & 61 & Li H. & 79 \\
\hline Kaušitz J. & 86 & Li R. & 79 \\
\hline Kawakami S. & 109,107 & Li Yan-Jie & 96 \\
\hline Kazimirsky A.N. & 106 & Linder S. & 13 \\
\hline Kehlen A. & 122 & Lindgren $\mathrm{T}$. & 76 \\
\hline Kennedy A. & 27 & Lindner I. & 19 \\
\hline Khwaja F. H. & 3 & Lingenhel A. & 70,71 \\
\hline Kitahara M. & 109 & Lipska L. & 118 \\
\hline Kithier K. & 4 & Liu Guang-Chao & 96 \\
\hline Kizek R. & $43,44,79,123,124$ & Lobl-Lavi L & 81 \\
\hline Kjell N. & 65 & Loening S.A. & 119 \\
\hline Klee G. G. & 34 & Löfroth P.O. & 76 \\
\hline Kleibl Z. & 83 & Logoteta M. & 80 \\
\hline Kleiblová P. & 83 & Loidl W. & 70 \\
\hline
\end{tabular}




\begin{tabular}{|c|c|c|c|}
\hline Lottspeich F. & 47 & Nahum K.D. & 119 \\
\hline Lüftner D. & 22 & Nakae Y. & 124 \\
\hline Lustig M. & 59 & Nakielna J. & 79 \\
\hline Lüth P. & 71 & Nap M. & 21,37 \\
\hline Luxemburger U; & 73 & Nasca M. & 28 \\
\hline Lyubimova N. & 101 & Němečková A. & 4 \\
\hline Ma Yuan-Fang & 96 & Nicolini A & 74 \\
\hline Malickova K. & 111 & Nielsen HJ & 47 \\
\hline Manabe K. & 107 & Nikiforenko J. & 96 \\
\hline Mand’áková P. & 9 & Nilsson O. & $95,97,102,103$ \\
\hline Mang T. & 45 & Nishina S.I. & 105 \\
\hline Manhart S. & 122 & Nisman B. & 11,102 \\
\hline Marafioti T. & 10 & Nojima M & 82,23 \\
\hline Marek J & 26 & Nordlund M. & 103 \\
\hline Mares V & 26 & Norman R & 15 \\
\hline Markowska J. & 13 & Nouso K. & 107 \\
\hline Marrades R. & 12 & Novotný J. & 83 \\
\hline Marshutina N.V. & 105 & Nowrousian M. R. & 57 \\
\hline Martens J. W.M. & 6 & O’Brien J. F. & 34 \\
\hline Martinez V. L. & 72 & Oehr P. & $29,29,31$ \\
\hline Maruyama R. & 73,78 & Ogino $\mathrm{T}$. & 107 \\
\hline Masopust J. & 4 & Öhrvik A. & 103 \\
\hline Matsumoto N. & 125 & Olivier P.A. & 114 \\
\hline Matsune T. & 85 & Olsen K.H. & 65 \\
\hline Matthes G. & 19 & Olson G. & 56 \\
\hline McElveen J; & 73 & Olsson M. & 103 \\
\hline McGing $\mathrm{P}$. & 13 & Orenes-Piñero E. & 125 \\
\hline Meister M. & 101 & Otto $\mathrm{Ch}$ & 17 \\
\hline Menashe B.E. & 39 & Otto Ch. & 18 \\
\hline Mersalian G. & 108 & Pagliuzzi M. & 80 \\
\hline Messina G. & 121 & Pahisa J. & 21 \\
\hline Metzgar M. H. & 10 & Palme S. & 19 \\
\hline Meyer H.A. & 119 & Palou J. & 126 \\
\hline Micca Gi. & 112 & Pantaleo G. & 39 \\
\hline Michalová K & 10 & Parnham A. & 112 \\
\hline Miller C. & 108 & Paterson J. & 10 \\
\hline Miller M. C. & 67 & Paus E. & 14,103 \\
\hline Mishunina M.P. & 105 & Payne S. & 51 \\
\hline Model F. & 51 & Peretz T. & 11,102 \\
\hline Mokhosoev I. & 99 & Perez R. & 119 \\
\hline Moldogazieva N.T. & $99,107,106$ & Pestlin G. & 19 \\
\hline Molina R. & $12,21,89,100,100$ & Pfeiffer Z. & 110 \\
\hline Molinam R. & 13 & Pfetzer N. & 17 \\
\hline Molnar B. & 46,80 & Philipp M. & 101 \\
\hline Monroe J. G. & 10 & Philippe M. & $113,113,114$ \\
\hline Moore R. G. & 108,67 & Piao W. & 79 \\
\hline Mori M & 82 & Pienkowski T. & 93 \\
\hline Moro R. & $92,92,92,101$ & Pietrobon M. & 80 \\
\hline Mrazkova P. & 30 & Pichler B. & 90 \\
\hline Mrhalová M. & 9 & Pine A. & 84 \\
\hline Mrkvan T. & $33,84,86,88,88$ & Platt D. & 119 \\
\hline Muelle T. & 70 & Plocova D. & 84,33 \\
\hline Mughal P. & 112 & Polivkova V. & 12,57 \\
\hline Mühling B. & 18 & Poryadin G.V. & 106 \\
\hline Muley T. & 101 & Possinger K. & 22 \\
\hline Mullier F. & 113,113 & Postma E. & 21 \\
\hline Muñoz M. & 21 & Prazakova M. & $30,104,57$ \\
\hline Murakami A. & 78 & Prazkova M. & 12 \\
\hline Muravsky V. & 19 & Priedite V. & 96 \\
\hline Murovska M. & 111,121 & Prokopenko P. & 90,104 \\
\hline Murray A. & 69,73 & Prusa R. & $43,44,79,123,124$ \\
\hline \multirow[t]{2}{*}{ Nagel D. } & $11,48,62,62,63,70,74,93$ & Puig S. & 89 \\
\hline & 94,110 & Qi Yi-Jun & 96 \\
\hline
\end{tabular}




\begin{tabular}{|c|c|}
\hline Quibriac M. & 89 \\
\hline Rádl J. & 4 \\
\hline Rahfeld J. & 122 \\
\hline Raith H. & 62 \\
\hline Ramirez J. & 91 \\
\hline Ramu N. & 102 \\
\hline Raspor B. & 42 \\
\hline Raubitschek A. & 72 \\
\hline Rauhut E. & 57 \\
\hline Recker F. & 118 \\
\hline Reinmiedl J. & 74 \\
\hline Reischl G. & 90 \\
\hline Riedinger J.M. & 89 \\
\hline Rihova B. & 84 \\
\hline Riklund K. Å. & 76 \\
\hline Risse B. & 19 \\
\hline Rittenhouse H. & 50,24 \\
\hline Robertson J. & 69,84 \\
\hline Robertson JFR & 73 \\
\hline Roger M. P. & $114,115,115,116,117$ \\
\hline Röijer E. & 102 \\
\hline Romanova $\mathrm{T}$. & 96 \\
\hline Romero P. & 32 \\
\hline Roobol M. & 49 \\
\hline Rossi G. & 74 \\
\hline Roth H.J. & 45 \\
\hline Rubach M. & 93 \\
\hline Rubio J. S. & 72 \\
\hline Rupert K. & 12,57 \\
\hline Russell E. & 91 \\
\hline Russell J. & 91 \\
\hline Rysinska A. & 93 \\
\hline Ř́hová B. & $33,77,81,85,86,88,88$ \\
\hline Safranek J. & 104 \\
\hline Sahin U; & 73 \\
\hline Sakaguchi K. & 105 \\
\hline Sakai H. & 78 \\
\hline Sakata I. & 124 \\
\hline Salamone M. & 28 \\
\hline Salgia R. & 28 \\
\hline Salmasi J.M. & 106 \\
\hline Salo T. & 87 \\
\hline Sandri M.T. & 112 \\
\hline Sanchez-Carbayo M. & $72,126,125$ \\
\hline Sanislo L. & 86 \\
\hline Sardesai N.Y. & 108 \\
\hline Sasaki S. & 85 \\
\hline Sasek L. & 56 \\
\hline Scopelliti M. & 80 \\
\hline Seckl M. J & 16 \\
\hline Sedlák J. & 86 \\
\hline Sedo A & 26 \\
\hline Seidel D. & 70,74 \\
\hline Seiler D. & 118 \\
\hline Seitz H. K. & 1 \\
\hline Sell S. & 2 \\
\hline Semjonow A. & 52 \\
\hline Sergeeva N.S. & 105 \\
\hline Serrano A. & 72,125 \\
\hline Shelepova V. & 90,104 \\
\hline Shibaguchi H. & 124 \\
\hline Shinomura Y. & $82,23,73,79,85$ \\
\hline Shiraha H. & 105 \\
\hline
\end{tabular}

Shiratori Y. $\quad 105$

Shively J. E. 90, 72

Shyroky V. 95

Scharpé S. $\quad 26$

Schaumlöffel D. 43

Scheiber K. 70

Schenk-Braat E. $\quad 51$

Schmid R. 101

Schmidt J. $\quad 56$

Schmidt M. $\quad 18$

Schmidt-Gayk H. $\quad 45$

Schneider J. 101

Schröder F. 49

Schubert P. $\quad 17$

Schumacher M. $\quad 61$

Skates S 67

Sklenáŕ J. 77

Skog S. $\quad 55$

Sledziewski A. $\quad 51$

Sokolov V.V. $\quad 97$

Sorsa T. $\quad 87$

Spelsberg F. $\quad 48,94$

Spisák S. 80

Spisakova M. $\quad 12,57$

Steiner H. 70

Steinhoff M. $\quad 108$

Steinitz M 81

Stenman U.H. $\quad 14,62,117,16,15$

Stephan C 119

Stieber P 47, 11, 13, 48, 62, 62, 63, 70, 71,

Stief Ch. G. $\quad 106$

Stigbrand T. $\quad 76$

Stork J. $\quad 122$

Stremenova J 26

Strittmatter F. $\quad 106$

Strohalm J. $\quad 33,77,81,84,85,86,88$

Strouhal E. $\quad 4$

Stroupe S. $\quad 91$

Strupova L. $\quad 118$

Sturgeon C 15, 25, 13, 14

Subr V. 84

Sugino N. $\quad 78$

Sultanova A. $\quad 121$

Suojanen J. $\quad 87$

Sutnar A. $\quad 12,57$

Suzuki H. $\quad 82,23,73,78$

Suzuki M. $\quad 105$

Svobodova S. $\quad 12,57$

Sykorova E. 43

Szpunar J. 43

Š́rová M. 33, 86

Šubr V. 33, 77

Tagirova A.K. 106

Tagliapietra A. $\quad 80$

Takaoka N. $\quad 105$

Takeda K. $\quad 78$

Taketa K. $\quad 107$

Tanaka N. $\quad 107$

Tanaka S. $\quad 105$

Tanner P. $\quad 117$

Tarapacz J. $\quad 99$

Tarasawa I. $\quad 78$ 


\begin{tabular}{|c|c|c|c|}
\hline Tatarinov Y. & 107 & Velasco M. & 21 \\
\hline Taubert H. & 122 & Verch T. & $67,108,108$ \\
\hline Telegina L.V. & 97 & Vesco V. & 28 \\
\hline Terentiev A. & $106,90,99,104,107$ & Větvička D. & 88 \\
\hline Tesarova P. & 111 & Vinolas N. & 12 \\
\hline Thiede A. & 18 & Visokai V. & 118 \\
\hline Thierolf M. & 19 & von Pawel J. & 62 \\
\hline Thomas J. & 110 & Votava $\mathrm{T}$. & 56 \\
\hline Thompson I. M. & 60 & Vrzalová J. & 109 \\
\hline Thornberry N. A. & 26 & Waidelich R. & 106 \\
\hline Tchemeris G. & 95 & Wang N. & 55 \\
\hline Tcherkassova J. & $92,92,101$ & Wang Y. & 79 \\
\hline Tobias J. W. & 10 & Warren D. J. & 65 \\
\hline Tokino T & 82 & Weiss G. & 51 \\
\hline Tomala J. & 85,88 & Wermann M. & 122 \\
\hline Topolcan O. & $12,30,56,57,104,109$ & Wetterauer U & 68,90 \\
\hline Torne A. & 21 & Whiteside T. L. & 32 \\
\hline Tóth K. & 80 & Whiting F. & 84 \\
\hline Toupalik P. & 44 & Wiefelspütz J. & 57 \\
\hline Toyota M. & $82,23,73,78$ & Wiewrodt R; & 73 \\
\hline Tran L & 108 & Wicha M. & 54 \\
\hline Traschütz Ch. & 101 & Wilde J. & 17 \\
\hline Treska V. & 12,57 & Wittig R. & 17 \\
\hline Tribukait B. & 55 & Wlodarska . & 9 \\
\hline Trnkova L. & 123,124 & Wójcik E. & 99 \\
\hline Tsao George SW & 96 & Wolf P. & 68,90 \\
\hline Tsuboi S. & 107 & Woodard K. & 69 \\
\hline Tsujisaki M. & 85 & Woolston C. & 84 \\
\hline Tulassay Z. & 80 & Yagi T. & 107 \\
\hline Türeci Ö; & 73 & Yakubovskaya R.I. & 97 \\
\hline Tuttle A. & 56 & Yamamoto H. & 79 \\
\hline Ueda N. & 105 & Yang & 7 \\
\hline Ulbrich K. & $33,77,81,84,85,86,88,88$ & Yasui H. & 23,78 \\
\hline Umeda Y. & 107 & Yoshimura T. & 109 \\
\hline Untch M. & 110 & Yu D. & 10 \\
\hline Urrutia M. & 126 & Zahlava J. & 30 \\
\hline Usui S. & 76 & Zalupski M. & 119 \\
\hline Uziely B. & 11 & Zemanová Z. & 10 \\
\hline Vajtr D. & 44 & Zenzmair Ch. & 14 \\
\hline Valik D. & 36 & Zhao X. & 125 \\
\hline van Dalen A. & 13 & Zitka O. & 43,123 \\
\hline van den Bergh R. & 49 & Zolg W. & 19 \\
\hline van Meir E. G. & 3 & Zomer E. & 119 \\
\hline Varaas $\mathrm{T}$. & 65,103 & zur Hausen A. & 17 \\
\hline
\end{tabular}

